Supporting Information

\title{
Total Syntheses of Aturanosides A and B
}

Yingjie Wang ${ }^{\mathrm{a}}$, Biao Yua, ${ }^{\mathrm{a}, \mathrm{b}}$ *

${ }^{a}$ State Key Laboratory of Bioorganic and Natural Products Chemistry, Center for Excellence in Molecular Synthesis, Shanghai Institute of Organic Chemistry, University of Chinese Academy of Sciences, Chinese Academy of Sciences, 345 Lingling Road, Shanghai 200032, China

b School of Chemistry and Materials Science, Hangzhou Institute for Advanced Study, University of Chinese Academy of Sciences, 1 Sub-lane Xiangshan, Hangzhou 310024, China

\section{Table of Contents}

I Experimental Procedures and Spectroscopic Data

II Comparison of the Spectroscopic Data of the Authentic and Synthetic Aturanosides

III References

IV ${ }^{1} \mathrm{H}$ and ${ }^{13} \mathrm{C}$ NMR Spectra of Compounds 


\section{Experimental Procedures and Spectroscopic Data}

\section{General procedure}

All reactions were carried out under an argon atmosphere with dry solvents under anhydrous conditions, unless otherwise noted. Tetrahydrofuran (THF) was distilled immediately before use from sodium. Methylene chloride $\left(\mathrm{CH}_{2} \mathrm{Cl}_{2}\right), N, N$-dimethylformamide (DMF), dimethyl sulfoxide (DMSO), tetramethylethylenediamine (TMEDA), and toluene were dried with activated Linde types $4 \AA$ molecular sieves and stored under an argon atmosphere. Methanol $(\mathrm{MeOH})$ was dried with activated Linde types $3 \AA$ molecular sieves and stored under an argon atmosphere. Reagents were purchased at the highest commercial quality and used without further purification, unless otherwise stated. Solvents for chromatography were used as supplied by Adamas-beta ${ }^{\circledR}$. Reactions were monitored by thin layer chromatography (TLC) carried out on MilliporeSigma glass TLC plates (silica gel 60 coated with $\mathrm{F}_{254}, 250 \mu \mathrm{m}$ ) using $\mathrm{UV}$ light for visualization and aqueous ammonium cerium nitrate/ammonium molybdate or ethanol solution of $0.1 \%(\mathrm{v} / \mathrm{v}) 3$-methoxyphenol and $2.5 \%$ (v/v) sulfuric acid as developing agent. SiliaFlash ${ }^{\circledR} \mathrm{P} 60$ silica gel (particle size: $40-63 \mu \mathrm{m}$, pore size: $60 \AA$ A) was used for flash column chromatography. NMR spectra were recorded on an Agilent DD2 $500 \mathrm{MHz}$ or a Bruker Avance III HD $600 \mathrm{MHz}$ NMR spectrometer. The spectra were calibrated by using residual undeuterated solvents (for ${ }^{1} \mathrm{H}$ NMR) and deuterated solvents (for ${ }^{13} \mathrm{C} \mathrm{NMR}$ ) as internal references: $\mathrm{CHCl}_{3}\left(\delta_{\mathrm{H}}=7.26 \mathrm{ppm}\right)$ and $\mathrm{CDCl}_{3}\left(\delta_{\mathrm{C}}=77.16 \mathrm{ppm}\right)$; $\mathrm{CHD}_{2} \mathrm{OD}\left(\delta_{\mathrm{H}}=3.31 \mathrm{ppm}\right)$ and methanol- $d_{4}\left(\delta_{\mathrm{C}}=49.00 \mathrm{ppm}\right)$; acetone- $d_{5}\left(\delta_{\mathrm{H}}=2.05 \mathrm{ppm}\right)$ and acetone- $d_{6}\left(\delta_{\mathrm{C}}=29.84 \mathrm{ppm}\right)$; DMSO- $d_{5}\left(\delta_{\mathrm{H}}=2.50 \mathrm{ppm}\right)$ and DMSO- $d_{6}\left(\delta_{\mathrm{C}}=39.52 \mathrm{ppm}\right)$. The following abbreviations are used to designate multiplicities: $\mathrm{s}=$ singlet, $\mathrm{d}=$ doublet, $\mathrm{t}=$ triplet, $\mathrm{q}=$ quartet, $\mathrm{m}=$ multiplet, $\mathrm{br}=$ broad. High-resolution mass spectra $(\mathrm{HRMS})$ were recorded on a Bruker maXis 4G (ESI-TOF) mass spectrometer. Specific rotations were recorded on an Anton Paar MPC 5500 polarimeter. 


\section{Experimental Procedures and Spectroscopic Data}
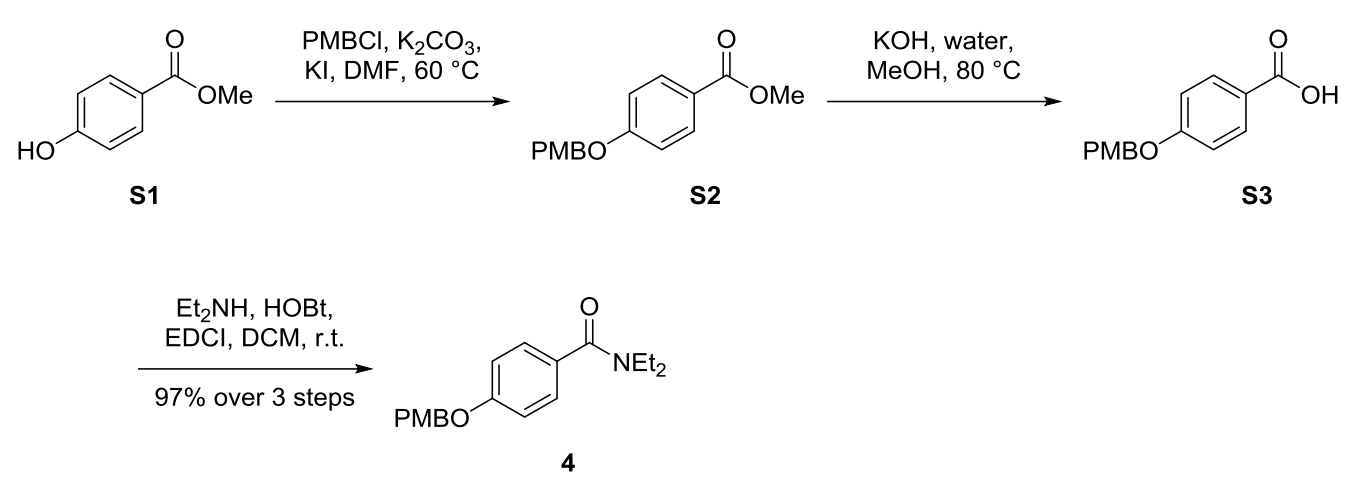

Compound 4. To a stirred solution of methyl 4-hydroxybenzoate S1 (30.4 g, $200 \mathrm{mmol})$ in DMF (200 mL) was sequentially added $\mathrm{K}_{2} \mathrm{CO}_{3}(110 \mathrm{~g}, 800 \mathrm{mmol})$, KI (3.30 g, $\left.20.0 \mathrm{mmol}\right)$, and 4-methoxybenzyl chloride $(30.0 \mathrm{~mL}, 220 \mathrm{mmol})$. The mixture was heated with heating mantle to $60{ }^{\circ} \mathrm{C}$ and stirred for $2 \mathrm{~h}$. The mixture was cooled to room temperature, diluted with water (4.0 L), and stirred for $30 \mathrm{~min}$. The suspension was filtrated. The filter cake was washed with water and dried under vacuum to give crude $\mathbf{S 2}$ as a white solid. The crude $\mathbf{S} \mathbf{2}$ was used directly in the next step without further purification.

The crude $\mathbf{S 2}$ was dispersed in methanol $(200 \mathrm{~mL})$, and then aq. KOH solution $(28.0 \mathrm{~g}$, $500 \mathrm{mmol}$, dissolved in $200 \mathrm{~mL}$ water) was added. The mixture was heated with heating mantle to $80{ }^{\circ} \mathrm{C}$ and stirred for $1 \mathrm{~h}$. The mixture was cooled to $0{ }^{\circ} \mathrm{C}$, to which was slowly added aq. $\mathrm{HCl}$ solution $(6.0 \mathrm{M})$ until $\mathrm{pH}=4$, then diluted with water $(2.0 \mathrm{~L})$. The suspension was filtrated. The filter cake was washed with water and dried under vacuum to give crude $\mathbf{S 3}$ as a white solid. The crude $\mathbf{S 3}$ was used directly in the next step without further purification.

The crude $\mathbf{S 3}$ was dissolved in $\mathrm{CH}_{2} \mathrm{Cl}_{2}(1.0 \mathrm{~L})$ and 1-hydroxybenzotriazole $(40.5 \mathrm{~g}, 300$ mmol) was added. The mixture was cooled to $0{ }^{\circ} \mathrm{C}$, then $\mathrm{Et}_{2} \mathrm{NH}(41.0 \mathrm{~mL}, 400 \mathrm{mmol})$ and 1ethyl-3-(3-dimethylaminopropyl)carbodiimide hydrochloride (46.0 g, $240 \mathrm{mmol}$ ) were added. The mixture was stirred at room temperature for $36 \mathrm{~h}$. The mixture was concentrated under vacuum. The residue was added into water $(1.0 \mathrm{~L})$ and stirred for $30 \mathrm{~min}$. The suspension was filtrated. The filter cake was sequentially washed with aq. $\mathrm{NaOH}$ solution $(1.0 \mathrm{M})$, aq. $\mathrm{HCl}$ solution (1.0 M), water, a small amount of cold ethanol, and petroleum ether. Then the residue 
was dried under vacuum to afford 4 as a white solid (60.5 g, 97\% over 3 steps).

Compound S2: ${ }^{1} \mathrm{H}$ NMR (500 MHz, $\left.\mathrm{CDCl}_{3}\right) \delta$ 8.01-7.97 (m, 2H), 7.38-7.33 (m, 2H), 7.00-6.96 (m, 2H), 6.94-6.91 (m, 2H), $5.04(\mathrm{~s}, 2 \mathrm{H}), 3.88(\mathrm{~s}, 3 \mathrm{H}), 3.82(\mathrm{~s}, 3 \mathrm{H}),{ }^{13} \mathrm{C}$ NMR $(126$ $\left.\mathrm{MHz}, \mathrm{CDCl}_{3}\right) \delta 167.0,162.7,159.8,131.7,129.4,128.4,122.9,114.6,114.2,70.1,55.5,52.0$ HRMS (ESI-TOF) m/z [M + Na] ${ }^{+}$calcd for $\mathrm{C}_{16} \mathrm{H}_{16} \mathrm{O}_{4} \mathrm{Na}^{+} 295.0941$, found 295.0944.

Compound S3: ${ }^{1} \mathrm{H}$ NMR (500 MHz, DMSO- $\left.d_{6}\right) \delta$ 7.90-7.84 (m, 2H), 7.42-7.36 (m, 2H), 7.08-7.02 (m, 2H), 6.98-6.93 (m, 2H), $5.08(\mathrm{~s}, 2 \mathrm{H}), 3.76(\mathrm{~s}, 3 \mathrm{H}),{ }^{13} \mathrm{C}$ NMR (126 MHz, DMSO$\left.d_{6}\right) \delta 167.3,161.6,159.1,131.2,129.7,128.5,114.4,113.9,69.2,55.1 ;$ HRMS (ESI-TOF) m/z $[\mathrm{M}+\mathrm{Na}]^{+}$calcd for $\mathrm{C}_{15} \mathrm{H}_{14} \mathrm{O}_{4} \mathrm{Na}^{+} 281.0784$, found 281.0791 .

Compound 4: ${ }^{1} \mathrm{H}$ NMR (500 MHz, $\left.\mathrm{CDCl}_{3}\right) \delta$ 7.37-7.32 (m, 4H), 6.98-6.94 (m, 2H), 6.94$6.90(\mathrm{~m}, 2 \mathrm{H}), 5.00(\mathrm{~s}, 2 \mathrm{H}), 3.82(\mathrm{~s}, 3 \mathrm{H}), 3.41$ (brs, 4H), 1.18 (brs, 6H); ${ }^{13} \mathrm{C}$ NMR $(126 \mathrm{MHz}$, $\left.\mathrm{CDCl}_{3}\right) \delta 171.3,159.7,159.7,129.8,129.4,128.8,128.3,114.7,114.2,70.0,55.5 ;$ HRMS (ESITOF) $\mathrm{m} / \mathrm{z}[\mathrm{M}+\mathrm{Na}]^{+}$calcd for $\mathrm{C}_{19} \mathrm{H}_{23} \mathrm{NO}_{3} \mathrm{Na}^{+} 336.1570$, found 336.1573 .
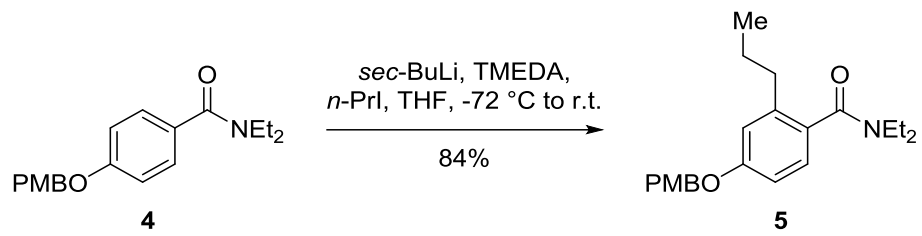

Compound 5. To a stirred solution of 4 (313 mg, $1.00 \mathrm{mmol})$ and TMEDA (300 $\mu \mathrm{L}, 2.00 \mathrm{mmol})$ in THF (10 mL) was added sec-BuLi (1.15 mL, 1.3 M in hexane, $1.50 \mathrm{mmol})$ dropwise under argon at $-72{ }^{\circ} \mathrm{C}$. The resulting mixture was stirred at that temperature for $1 \mathrm{~h}$ before 1 iodopropane $(390 \mu \mathrm{L}, 4.00 \mathrm{mmol})$ was added dropwise. The mixture was stirred at $-72{ }^{\circ} \mathrm{C}$ for $30 \mathrm{~min}$, and was then warm to room temperature for $30 \mathrm{~min}$ before it was quenched with saturated aq. $\mathrm{NH}_{4} \mathrm{Cl}(0.5 \mathrm{~mL})$. The mixture was diluted with EtOAc, washed with brine, dried over anhydrous $\mathrm{Na}_{2} \mathrm{SO}_{4}$, filtered, and concentrated under vacuum. The residue was purified by silica gel chromatography (petroleum ether/EtOAc, 2:1) to afford 5 as a white solid (300 mg, 84\% yield): ${ }^{1} \mathrm{H}$ NMR $\left(500 \mathrm{MHz}, \mathrm{CDCl}_{3}\right) \delta$ 7.37-7.33 (m, 2H), $7.08(\mathrm{~d}, J=8.3 \mathrm{~Hz}, 1 \mathrm{H}), 6.94-$ $6.90(\mathrm{~m}, 2 \mathrm{H}), 6.84(\mathrm{~d}, J=2.5 \mathrm{~Hz}, 1 \mathrm{H}), 6.79(\mathrm{dd}, J=8.3,2.5 \mathrm{~Hz}, 1 \mathrm{H}), 4.98(\mathrm{~s}, 2 \mathrm{H}), 3.92-3.70$ (m, 4H), 3.31 (brs, 1H), 3.13 (brs, 2H), 2.53 (brs, 2H), 1.61 (brs, 2H), 1.24 (t, $J=6.8 \mathrm{~Hz}, 3 \mathrm{H})$, 
$1.04(\mathrm{t}, J=6.8 \mathrm{~Hz}, 3 \mathrm{H}), 0.93(\mathrm{t}, J=7.3 \mathrm{~Hz}, 3 \mathrm{H}) ;{ }^{13} \mathrm{C} \mathrm{NMR}\left(126 \mathrm{MHz}, \mathrm{CDCl}_{3}\right) \delta 171.0,159.6$, 159.0, 140.8, 129.7, 129.4, 128.9, 127.0, 116.0, 114.1, 111.9, 69.9, 55.4, 42.9, 38.7, 35.4, 24.0, 14.3, 14.1, 12.8; HRMS (ESI-TOF) $\mathrm{m} / \mathrm{z}[\mathrm{M}+\mathrm{Na}]^{+}$calcd for $\mathrm{C}_{22} \mathrm{H}_{29} \mathrm{NO}_{3} \mathrm{Na}^{+}$378.2040, found 378.2043.
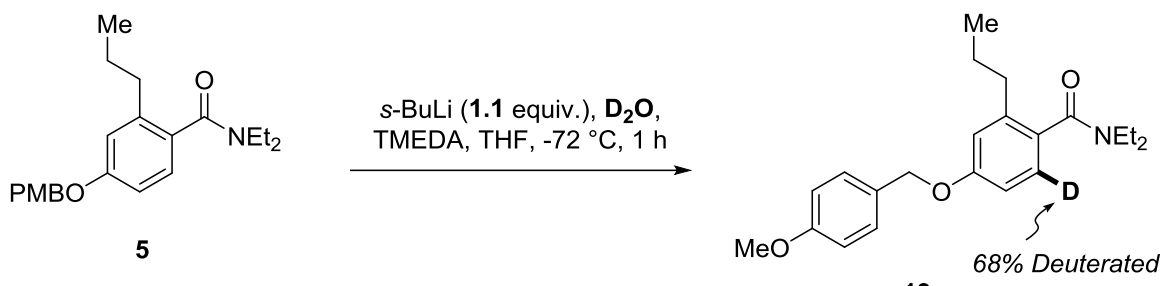

13

Compound 13. To a stirred solution of $5(73.0 \mathrm{mg}, 0.20 \mathrm{mmol})$ and TMEDA ( $90 \mu \mathrm{L}, 0.60$ $\mathrm{mmol})$ in THF (2.0 mL) was added sec-BuLi $(0.17 \mathrm{~mL}, 1.3 \mathrm{M}$ in hexane, $0.22 \mathrm{mmol})$ dropwise under argon at $-72{ }^{\circ} \mathrm{C}$. The mixture was stirred at that temperature for $1 \mathrm{~h}$ before it was quenched with deuterium oxide. The resulting mixture was diluted with EtOAc and washed with aq. $\mathrm{HCl}(1.0 \mathrm{M})$, saturated aq. $\mathrm{NaHCO}_{3}$, and brine sequentially, then dried over anhydrous $\mathrm{Na}_{2} \mathrm{SO}_{4}$, filtered, and concentrated under vacuum to afford crude 13 (69.0 mg, 95\%): ${ }^{1} \mathrm{H} \mathrm{NMR}$ $\left(500 \mathrm{MHz}, \mathrm{CDCl}_{3}\right) \delta 7.38-7.32(\mathrm{~m}, 2 \mathrm{H}), 7.07(\mathrm{~d}, J=8.4 \mathrm{~Hz}, \underline{\mathbf{0 . 3 2 H}}), 6.94-6.88(\mathrm{~m}, 2 \mathrm{H}), 6.84$ $(\mathrm{d}, J=2.6 \mathrm{~Hz}, 1 \mathrm{H}), 6.81-6.76(\mathrm{~m}, 1 \mathrm{H}), 4.97$ (s, 2H), 3.90-3.67 (m, 4H), 3.30 (brs, 1H), 3.203.05 (m, 2H), 2.52 (brs, 2H), 1.61 (brs, 2H), 1.23 (t, $J=7.1 \mathrm{~Hz}, 3 \mathrm{H}), 1.03$ (t, $J=7.2 \mathrm{~Hz}, 3 \mathrm{H})$, $0.93(\mathrm{t}, J=7.3 \mathrm{~Hz}, 3 \mathrm{H})$.
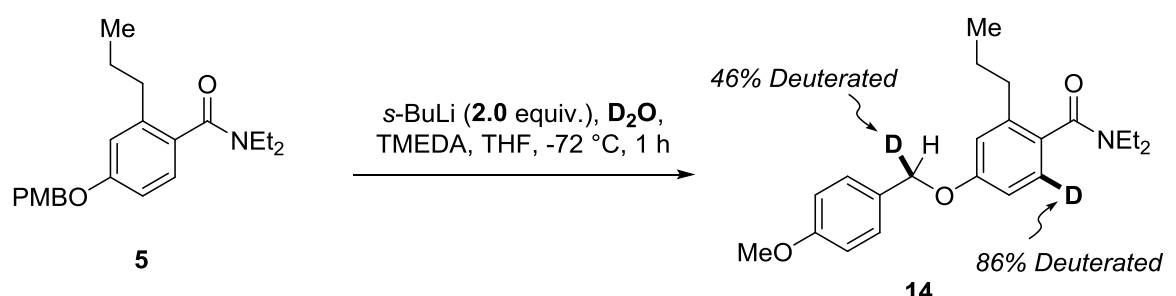

Compound 14. To a stirred solution of $5(36.0 \mathrm{mg}, 0.10 \mathrm{mmol})$ and TMEDA (66 $\mu \mathrm{L}, 0.30$ $\mathrm{mmol})$ in THF (1.0 mL) was added sec-BuLi $(0.15 \mathrm{~mL}, 1.3 \mathrm{M}$ in hexane, $0.20 \mathrm{mmol})$ dropwise under argon at $-72{ }^{\circ} \mathrm{C}$. The mixture was stirred at that temperature for $1 \mathrm{~h}$ before it was quenched with deuterium oxide. The resulting mixture was diluted with EtOAc and washed 
with aq. $\mathrm{HCl}(1.0 \mathrm{M})$, saturated aq. $\mathrm{NaHCO}_{3}$, and brine sequentially, then dried over anhydrous $\mathrm{Na}_{2} \mathrm{SO}_{4}$, filtered, and concentrated under vacuum to afford crude 14 (33.0 mg, 92\%): ${ }^{1} \mathrm{H} \mathrm{NMR}$ $\left(500 \mathrm{MHz}, \mathrm{CDCl}_{3}\right) \delta 7.37-7.33(\mathrm{~m}, 2 \mathrm{H}), 7.07(\mathrm{~d}, J=8.3 \mathrm{~Hz}, \underline{\mathbf{0 . 1 4 H}}), 6.93-6.89(\mathrm{~m}, 2 \mathrm{H}), 6.84$ $(\mathrm{d}, J=2.6 \mathrm{~Hz}, 1 \mathrm{H}), 6.80-6.77(\mathrm{~m}, 1 \mathrm{H}), 4.98-4.94(\mathrm{~m}, \underline{\mathbf{1 . 5 4 H}}), 3.85-3.76(\mathrm{~m}, 4 \mathrm{H}), 3.29$ (brs, 1H), 3.20-3.01 (m, 2H), 2.52 (brs, 2H), 1.61 (brs, 2H), 1.23 (t, $J=7.1 \mathrm{~Hz}, 3 \mathrm{H}), 1.03$ (t, $J=7.1$ $\mathrm{Hz}, 3 \mathrm{H}), 0.93(\mathrm{t}, J=7.3 \mathrm{~Hz}, 3 \mathrm{H})$.
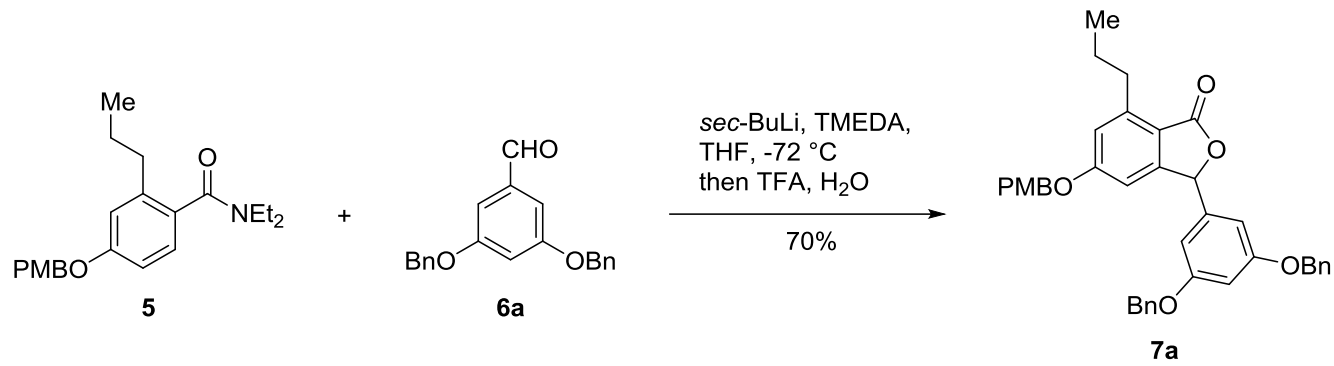

Compound 7a. To a stirred solution of 5 (3.56 g, $10.0 \mathrm{mmol})$ and TMEDA (3.00 mL, 20.0 $\mathrm{mmol})$ in THF $(70 \mathrm{~mL})$ was added $s e c-\mathrm{BuLi}(9.00 \mathrm{~mL}, 1.3 \mathrm{M}$ in hexane, $11.7 \mathrm{mmol})$ dropwise under argon at $-72{ }^{\circ} \mathrm{C}$. The resulting mixture was stirred at that temperature for $1 \mathrm{~h}$, then a solution of aldehyde 6a (4.78 g, $15.0 \mathrm{mmol}$, dissolved in $30 \mathrm{~mL}$ THF) was added dropwise in $30 \mathrm{~min}$. The mixture was stirred at $-72{ }^{\circ} \mathrm{C}$ for $1.5 \mathrm{~h}$ before it was quenched with water $(10.0$ $\mathrm{mL}$ ). The mixture was warm to room temperature and extracted with EtOAc three times. The combined organic phase was washed with aq. $\mathrm{HCl}(1.0 \mathrm{M})$, saturated aq. $\mathrm{NaHCO}_{3}$, and brine sequentially, and was then dried over anhydrous $\mathrm{Na}_{2} \mathrm{SO}_{4}$, filtered, and concentrated under vacuum.

The residue was dissolved in THF $(100 \mathrm{~mL})$, then water $(15.0 \mathrm{~mL})$ and trifluoroacetic acid (5.00 mL, $65.0 \mathrm{mmol})$ were added at room temperature. The mixture was stirred at $\mathrm{rt}$ for $15 \mathrm{~h}$ before it was quenched with aq. $\mathrm{NaOH}(1.0 \mathrm{M}, 60.0 \mathrm{~mL}, 60.0 \mathrm{mmol})$. The mixture was extracted with ether three times. The combined organic phase was washed with brine, dried over anhydrous $\mathrm{Na}_{2} \mathrm{SO}_{4}$, filtered, and concentrated under vacuum. The residue was purified by silica gel chromatography (petroleum ether/EtOAc, 10:1 to 5:1) to afford 7a as a white solid (4.21 g, 70\% yield): ${ }^{1} \mathrm{H}$ NMR (500 MHz, $\left.\mathrm{CDCl}_{3}\right) \delta$ 7.41-7.35 (m, 8H), 7.34-7.29 (m, 4H), 6.94-6.89 (m, 2H), $6.87(\mathrm{~d}, J=2.1 \mathrm{~Hz}, 1 \mathrm{H}), 6.60(\mathrm{t}, J=2.3 \mathrm{~Hz}, 1 \mathrm{H}), 6.59(\mathrm{dd}, J=2.2,0.9 \mathrm{~Hz}$, 
$1 \mathrm{H}), 6.52(\mathrm{~d}, J=2.2 \mathrm{~Hz}, 2 \mathrm{H}), 6.13(\mathrm{~s}, 1 \mathrm{H}), 5.04-4.97(\mathrm{~m}, 4 \mathrm{H}), 4.93(\mathrm{~s}, 2 \mathrm{H}), 3.81(\mathrm{~s}, 3 \mathrm{H}), 3.10$ (ddd, $J=13.4,8.5,6.7 \mathrm{~Hz}, 1 \mathrm{H}), 3.01(\mathrm{ddd}, J=13.5,8.4,6.8 \mathrm{~Hz}, 1 \mathrm{H}), 1.76-1.67$ (m, 2H), 1.00 $(\mathrm{t}, J=7.3 \mathrm{~Hz}, 3 \mathrm{H}) ;{ }^{13} \mathrm{C} \mathrm{NMR}\left(126 \mathrm{MHz}, \mathrm{CDCl}_{3}\right) \delta 170.2,163.8,160.4,159.9,153.1,146.4$, $139.5,136.6,129.6,128.8,128.3,127.9,127.7,117.8,115.2,114.2,106.0,105.2,102.5,81.1$, 70.4, 70.3, 55.5, 33.1, 24.0, 14.0; HRMS (ESI-TOF) $\mathrm{m} / \mathrm{z}[\mathrm{M}+\mathrm{Na}]^{+}$calcd for $\mathrm{C}_{39} \mathrm{H}_{36} \mathrm{O}_{6} \mathrm{Na}^{+}$ 623.2404, found 623.2398.

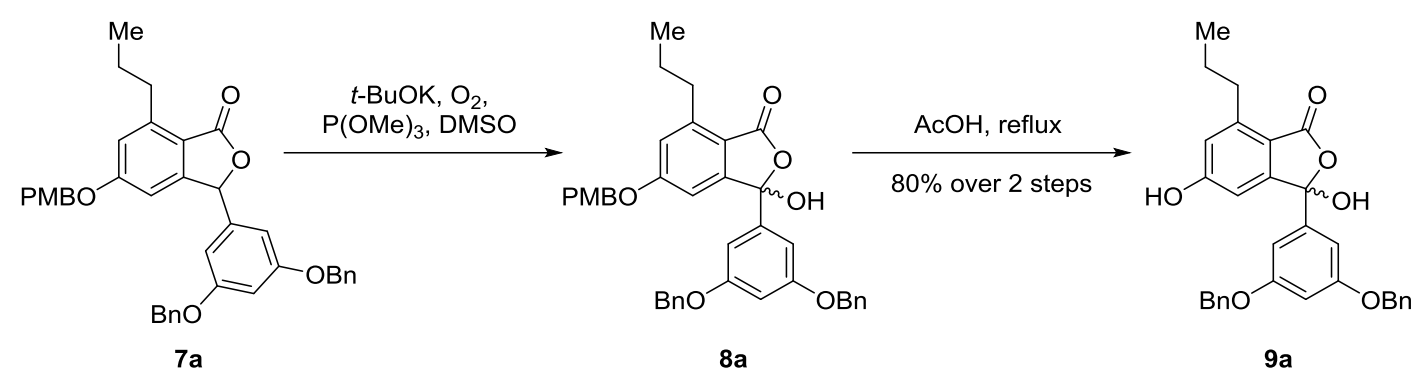

Compound 9a. To a stirred solution of 7a (300 mg, $0.50 \mathrm{mmol})$ and trimethyl phosphite (300 $\mu \mathrm{L}, 2.50 \mathrm{mmol})$ in DMSO $(2.50 \mathrm{~mL})$ was added potassium tert-butoxide (112 mg, $1.00 \mathrm{mmol})$ under oxygen at $20{ }^{\circ} \mathrm{C}$ cooling by water bath. The resulting red solution was stirred at that temperature until TLC indicated disappearance of the starting material. The colorless solution was quenched with aq. $\mathrm{HCl}(1.0 \mathrm{M})$ and extracted with EtOAc three times. The combined organic phase was washed with aq. $\mathrm{HCl}(1.0 \mathrm{M})$, saturated aq. $\mathrm{NaHCO}_{3}$, and brine sequentially, and was then dried over anhydrous $\mathrm{Na}_{2} \mathrm{SO}_{4}$, filtered, and concentrated under vacuum to give crude 8a as a white solid. The crude 8a was used directly in the next step without further purification.

The crude 8a was dissolved in acetic acid $(10.0 \mathrm{~mL})$ and refluxed for $5 \mathrm{~h}$. The resulting mixture was concentrated under vacuum. The residue was purified by silica gel chromatography (petroleum ether/EtOAc, 3:1 to 2:1) to afford 9a as a white solid (199 mg, 80\% yield).

Compound 8a: ${ }^{1} \mathrm{H}$ NMR (500 MHz, DMSO-d6) $\delta 8.39(\mathrm{~s}, 1 \mathrm{H}), 7.45-7.27(\mathrm{~m}, 12 \mathrm{H}), 7.00$ $(\mathrm{d}, J=2.1 \mathrm{~Hz}, 1 \mathrm{H}), 6.92(\mathrm{~d}, J=8.3 \mathrm{~Hz}, 2 \mathrm{H}), 6.87(\mathrm{~d}, J=2.1 \mathrm{~Hz}, 1 \mathrm{H}), 6.73-6.66(\mathrm{~m}, 3 \mathrm{H}), 5.13-$ $5.02(\mathrm{~m}, 6 \mathrm{H}), 3.73(\mathrm{~s}, 3 \mathrm{H}), 3.02-2.87(\mathrm{~m}, 2 \mathrm{H}), 1.68-1.57(\mathrm{~m}, 2 \mathrm{H}), 0.92(\mathrm{t}, J=7.3 \mathrm{~Hz}, 3 \mathrm{H}) ;{ }^{13} \mathrm{C}$ NMR (126 MHz, DMSO- $\left.d_{6}\right) \delta 167.8,163.5,159.5,159.2,154.0,144.9,142.5,136.8,130.0$, 
$128.4,128.0,127.9,127.7,118.0,114.7,113.8,106.1,104.7,104.0,101.9,69.8,69.4,55.1$, 32.4, 23.6, 13.7; HRMS (ESI-TOF) m/z $[\mathrm{M}+\mathrm{Na}]^{+}$calcd for $\mathrm{C}_{39} \mathrm{H}_{36} \mathrm{O}_{7} \mathrm{Na}^{+}$639.2353, found 639.2349 .

Compound 9a: ${ }^{1} \mathrm{H}$ NMR $\left(500 \mathrm{MHz}\right.$, acetone- $\left.d_{6}\right) \delta 7.46(\mathrm{~d}, J=7.3 \mathrm{~Hz}, 4 \mathrm{H}), 7.41-7.35(\mathrm{~m}$, 4H), $7.32(\mathrm{t}, J=7.3 \mathrm{~Hz}, 2 \mathrm{H}), 6.84(\mathrm{~d}, J=2.0 \mathrm{~Hz}, 1 \mathrm{H}), 6.82(\mathrm{~d}, J=2.3 \mathrm{~Hz}, 2 \mathrm{H}), 6.73(\mathrm{~d}, J=2.1$ $\mathrm{Hz}, 1 \mathrm{H}), 6.69(\mathrm{t}, J=2.2 \mathrm{~Hz}, 1 \mathrm{H}), 5.10(\mathrm{~s}, 4 \mathrm{H}), 3.00-2.94(\mathrm{~m}, 2 \mathrm{H}), 1.71-1.62(\mathrm{~m}, 2 \mathrm{H}), 0.96(\mathrm{t}$, $J=7.4 \mathrm{~Hz}, 3 \mathrm{H}) ;{ }^{13} \mathrm{C} \mathrm{NMR}\left(126 \mathrm{MHz}\right.$, acetone- $\left.d_{6}\right) \delta 168.6,163.6,161.0,146.6,143.6,138.1$, 129.3, 128.7, 128.5, 119.1, 108.1, 105.9, 102.8, 70.7, 33.6, 24.8, 14.1; HRMS (ESI-TOF) m/z $[\mathrm{M}+\mathrm{Na}]^{+}$calcd for $\mathrm{C}_{31} \mathrm{H}_{28} \mathrm{O}_{6} \mathrm{Na}^{+}$519.1778, found 519.1780.

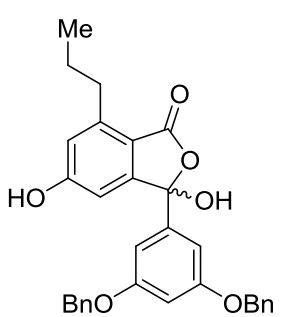

9a
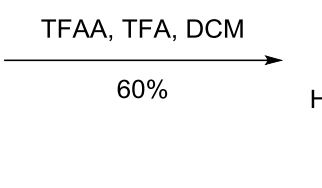

HO

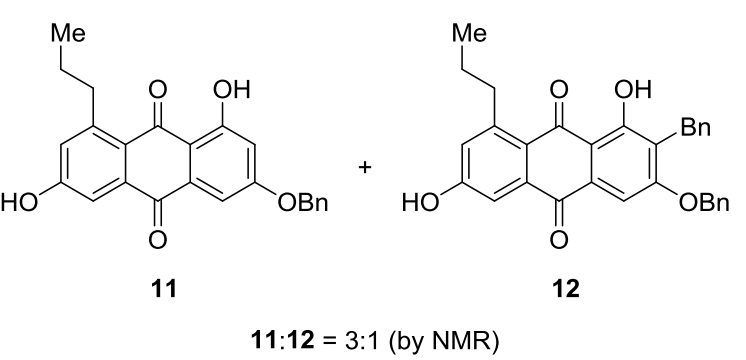

Compounds 11 and 12. To a stirred suspension of 9a (25.0 mg, $0.05 \mathrm{mmol})$ in $\mathrm{CH}_{2} \mathrm{Cl}_{2}(2.0$ $\mathrm{mL})$ were added trifluoroacetic anhydride $(0.14 \mathrm{~mL}, 1.0 \mathrm{mmol})$ and trifluoroacetic acid (1.0 $\mathrm{mL}$ ) under argon at room temperature. The mixture was stirred at that temperature for $1 \mathrm{~h}$. The resulting red solution was poured into saturated aq. $\mathrm{NaHCO}_{3}$ and extracted with EtOAc three times. The combined organic phase was washed with brine, dried over anhydrous $\mathrm{Na}_{2} \mathrm{SO}_{4}$, filtered, and concentrated under vacuum. The residue was purified by silica gel chromatography (petroleum ether/acetone, 5:1) to afford a mixture of 11 and $12(\mathbf{1 1} / \mathbf{1 2}=3: 1)$ as a yellow solid (13.0 mg, 60\% yield). The mixture of $\mathbf{1 1}$ and $\mathbf{1 2}$ was hardly separated by silica gel chromatography, it was therefore separated by HPLC (Agilent Eclipse XDB-C18 column, $75 \% \mathrm{CH}_{3} \mathrm{CN}+25 \%$ methanol).

Compound 11: ${ }^{1} \mathrm{H}$ NMR (600 MHz, acetone- $\left.d_{6}\right) \delta 13.37(\mathrm{~s}, 1 \mathrm{H}), 7.61(\mathrm{~d}, J=2.7 \mathrm{~Hz}, 1 \mathrm{H})$, 7.56-7.53 (m, 2H), 7.45-7.42 (m, 2H), 7.39-7.35 (m, 1H), $7.30(\mathrm{~d}, J=2.6 \mathrm{~Hz}, 1 \mathrm{H}), 7.12(\mathrm{~d}, J$ $=2.7 \mathrm{~Hz}, 1 \mathrm{H}), 6.86(\mathrm{~d}, J=2.6 \mathrm{~Hz}, 1 \mathrm{H}), 5.33(\mathrm{~s}, 2 \mathrm{H}), 3.23-3.19(\mathrm{~m}, 2 \mathrm{H}), 1.73-1.64(\mathrm{~m}, 2 \mathrm{H})$, $1.03(\mathrm{t}, J=7.3 \mathrm{~Hz}, 3 \mathrm{H}) ;{ }^{13} \mathrm{C}$ NMR $\left(151 \mathrm{MHz}\right.$, acetone- $\left.d_{6}\right) \delta 189.5,183.1,166.2,165.4,162.9$, 
$151.2,138.7,137.3,135.5,129.5,129.1,128.6,125.2,123.7,113.4,112.4,108.5,107.5,71.2$, 38.7, 24.8, 14.6; HRMS (ESI-TOF) $\mathrm{m} / \mathrm{z}[\mathrm{M}+\mathrm{Na}]^{+}$calcd for $\mathrm{C}_{24} \mathrm{H}_{20} \mathrm{O}_{5} \mathrm{Na}^{+}$411.1203, found 411.1204 .

Compound 12: ${ }^{1} \mathrm{H}$ NMR $\left(600 \mathrm{MHz}\right.$, acetone- $\left.d_{6}\right) \delta 13.75(\mathrm{~s}, 1 \mathrm{H}), 7.60(\mathrm{~d}, J=2.8 \mathrm{~Hz}, 1 \mathrm{H})$, 7.47-7.43 (m, 3H), 7.42-7.38 (m, 2H), 7.37-7.31 (m, 3H), 7.22 (t, J = 7.6 Hz, 2H), 7.16-7.12 (m, 1H), $7.10(\mathrm{~d}, J=2.7 \mathrm{~Hz}, 1 \mathrm{H}), 5.40(\mathrm{~s}, 2 \mathrm{H}), 4.13(\mathrm{~s}, 2 \mathrm{H}), 3.23-3.19(\mathrm{~m}, 2 \mathrm{H}), 1.74-1.64(\mathrm{~m}$, 2H), $1.03(\mathrm{t}, J=7.3 \mathrm{~Hz}, 3 \mathrm{H}) ;{ }^{13} \mathrm{C}$ NMR $\left(151 \mathrm{MHz}\right.$, acetone- $\left.d_{6}\right) \delta 190.0,183.1,163.1,162.8$, $162.6,151.2,141.1,138.6,137.4,133.4,129.7,129.4,128.99,128.96,128.5,126.7,125.1$, 124.5, 123.6, 113.5, 112.9, 103.5, 71.3, 38.7, 29.3, 24.8, 14.6; HRMS (ESI-TOF) m/z [M + H] $]^{+}$ calcd for $\mathrm{C}_{31} \mathrm{H}_{27} \mathrm{O}_{5}{ }^{+}$479.1853, found 479.1855 .
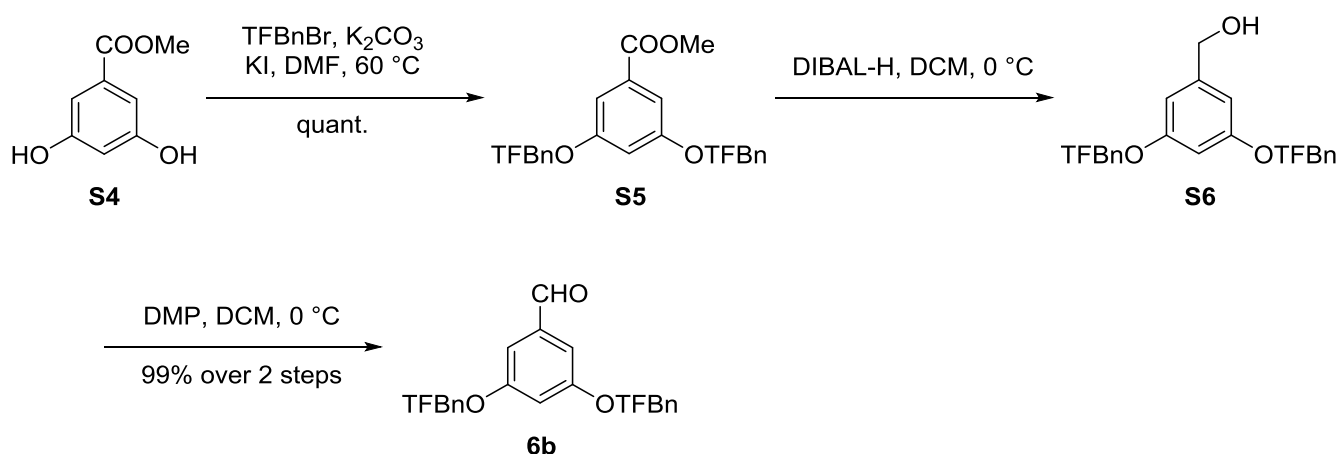

Compound 6b. To a stirred solution of methyl 3,5-dihydroxybenzoate S4 (8.40 g, 50.0 mmol) in DMF (50 mL) were sequentially added $\mathrm{K}_{2} \mathrm{CO}_{3}(28.0 \mathrm{~g}, 200 \mathrm{mmol}), \mathrm{KI}(4.20 \mathrm{~g}, 25.0 \mathrm{mmol})$, and 4-(trifluoromethyl)benzyl bromide $(25.0 \mathrm{~g}, 105 \mathrm{mmol})$. The mixture was heated with heating mantle to $60{ }^{\circ} \mathrm{C}$ for $3 \mathrm{~h}$. The mixture was cooled to room temperature, poured into water $(500 \mathrm{~mL})$, and extracted with EtOAc three times. The combined organic phase was washed with saturated aq. $\mathrm{NaHCO}_{3}$ and brine, dried over anhydrous $\mathrm{Na}_{2} \mathrm{SO}_{4}$, filtered, and concentrated under vacuum. The residue was purified by silica gel chromatography (petroleum ether/EtOAc, 9:1 to 5:1) to afford S5 as a white solid (24.2 g, quant.).

To a stirred solution of $\mathbf{S 5}(12.1 \mathrm{~g}, 25.0 \mathrm{mmol})$ in $\mathrm{CH}_{2} \mathrm{Cl}_{2}(125 \mathrm{~mL})$ was added diisobutylaluminium hydride $(60.0 \mathrm{~mL}, 1.0 \mathrm{M}$ in hexane, $60.0 \mathrm{mmol})$ under argon at $0{ }^{\circ} \mathrm{C}$. The mixture was stirred at that temperature for $1 \mathrm{~h}$ before it was quenched with saturated aq. 
potassium sodium tartrate. The mixture was extracted with $\mathrm{CH}_{2} \mathrm{Cl}_{2}$ three times, and the combined organic phase was washed with brine, dried over anhydrous $\mathrm{Na}_{2} \mathrm{SO}_{4}$, filtered, and concentrated under vacuum to afford crude S6 as a white solid. The crude S6 was used directly in the next step without further purification.

The residue $\mathbf{S 6}$ was dissolved in $\mathrm{CH}_{2} \mathrm{Cl}_{2}(250 \mathrm{~mL})$, cooled to $0{ }^{\circ} \mathrm{C}$, then Dess-Martin periodinane $(12.7 \mathrm{~g}, 30.0 \mathrm{mmol})$ was added. The mixture was stirred at that temperature for 2 h before it was quenched with saturated aq. $\mathrm{NaHCO}_{3}$ and saturated aq. $\mathrm{Na}_{2} \mathrm{~S}_{2} \mathrm{O}_{3}$ sequentially. The mixture was extracted with EtOAc three times, and the combined organic phase was washed with aq. $\mathrm{Na}_{2} \mathrm{~S}_{2} \mathrm{O}_{3}$ and brine, dried over anhydrous $\mathrm{Na}_{2} \mathrm{SO}_{4}$, filtered, and concentrated under vacuum. The residue was purified by silica gel chromatography (petroleum ether/EtOAc, 4:1) to afford $\mathbf{6 b}$ as a white solid (11.2 g, 99\% over 2 steps).

Compound S5: ${ }^{1} \mathrm{H}$ NMR (500 MHz, $\left.\mathrm{CDCl}_{3}\right) \delta 7.65(\mathrm{~d}, J=8.0 \mathrm{~Hz}, 4 \mathrm{H}), 7.55(\mathrm{~d}, J=8.0$ $\mathrm{Hz}, 4 \mathrm{H}), 7.31(\mathrm{~d}, J=2.4 \mathrm{~Hz}, 2 \mathrm{H}), 6.80(\mathrm{t}, J=2.4 \mathrm{~Hz}, 1 \mathrm{H}), 5.14(\mathrm{~s}, 4 \mathrm{H}), 3.92(\mathrm{~s}, 3 \mathrm{H}) ;{ }^{13} \mathrm{C} \mathrm{NMR}$ $\left(126 \mathrm{MHz} \mathrm{CDCl}_{3}\right) \delta 166.6,159.6,140.6(\mathrm{q}, J=1.4 \mathrm{~Hz}), 132.4,130.4(\mathrm{q}, J=32.4 \mathrm{~Hz}), 127.5$, $125.7(\mathrm{q}, J=3.8 \mathrm{~Hz}), 124.2(\mathrm{q}, J=272.6 \mathrm{~Hz}), 108.6,107.4,69.5,52.5 ;$ HRMS (ESI-TOF) m/z $[\mathrm{M}+\mathrm{Na}]^{+}$calcd for $\mathrm{C}_{24} \mathrm{H}_{18} \mathrm{~F}_{6} \mathrm{O}_{4} \mathrm{Na}^{+}$507.1001, found 507.0998.

Compound S6: ${ }^{1} \mathrm{H}$ NMR (500 MHz, $\left.\mathrm{CDCl}_{3}\right) \delta 7.64(\mathrm{~d}, J=8.0 \mathrm{~Hz}, 1 \mathrm{H}), 7.53(\mathrm{~d}, J=8.0$ $\mathrm{Hz}, 1 \mathrm{H}), 6.63(\mathrm{~d}, J=2.3 \mathrm{~Hz}, 1 \mathrm{H}), 6.52(\mathrm{t}, J=2.3 \mathrm{~Hz}, 0 \mathrm{H}), 5.11(\mathrm{~s}, 1 \mathrm{H}), 4.65(\mathrm{~s}, 1 \mathrm{H}) ;{ }^{13} \mathrm{C} \mathrm{NMR}$ $\left(126 \mathrm{MHz} \mathrm{CDCl}_{3}\right) \delta 159.9,143.9,141.0(\mathrm{q}, J=1.4 \mathrm{~Hz}), 130.3(\mathrm{q}, J=32.2 \mathrm{~Hz}), 127.5,125.7$ $(\mathrm{q}, J=3.8 \mathrm{~Hz}), 124.2(\mathrm{q}, J=272.5 \mathrm{~Hz}), 106.0,101.5,69.3,65.3 ;$ HRMS (ESI-TOF) m/z [M + $\mathrm{Na}]^{+}$calcd for $\mathrm{C}_{23} \mathrm{H}_{18} \mathrm{~F}_{6} \mathrm{O}_{3} \mathrm{Na}^{+} 479.1052$, found 479.1047 .

Compound 6b: ${ }^{1} \mathrm{H}$ NMR (500 MHz, $\left.\mathrm{CDCl}_{3}\right) \delta 9.91(\mathrm{~s}, 1 \mathrm{H}), 7.68-7.64(\mathrm{~m}, 4 \mathrm{H}), 7.57-7.54$ $(\mathrm{m}, 4 \mathrm{H}), 7.12(\mathrm{~d}, J=2.3 \mathrm{~Hz}, 2 \mathrm{H}), 6.85(\mathrm{t}, J=2.3 \mathrm{~Hz}, 1 \mathrm{H}), 5.17(\mathrm{~s}, 4 \mathrm{H}) ;{ }^{13} \mathrm{C} \mathrm{NMR}(126 \mathrm{MHz}$, $\left.\mathrm{CDCl}_{3}\right) \delta 191.6,160.2,140.3,138.8,130.6(\mathrm{q}, J=32.5 \mathrm{~Hz}), 127.6,125.8(\mathrm{q}, J=3.8 \mathrm{~Hz}), 124.2$ $(\mathrm{q}, J=272.1 \mathrm{~Hz}), 108.8,108.6,69.6 ; \mathrm{HRMS}(\mathrm{ESI-TOF}) \mathrm{m} / \mathrm{z}[\mathrm{M}+\mathrm{Na}]^{+}$calcd for $\mathrm{C}_{23} \mathrm{H}_{16} \mathrm{~F}_{6} \mathrm{O}_{3} \mathrm{Na}^{+}$477.0896, found 477.0897. 


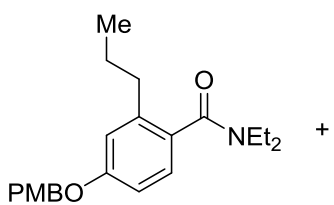

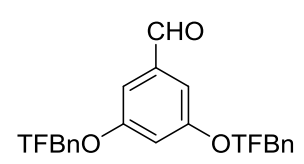

6b

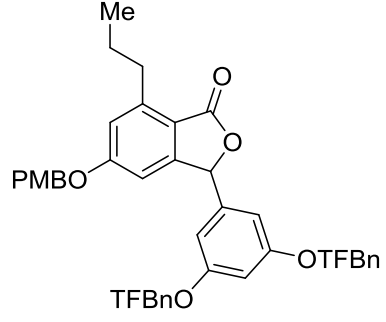

$7 b$

Compound 7b. To a stirred solution of 5 (2.75 g, $7.70 \mathrm{mmol})$ and TMEDA $(2.30 \mathrm{~mL}, 15.4$ $\mathrm{mmol})$ in THF $(60 \mathrm{~mL})$ was added $s e c-\mathrm{BuLi}(7.70 \mathrm{~mL}, 1.3 \mathrm{M}$ in hexane, $10.0 \mathrm{mmol})$ dropwise under argon at $-72{ }^{\circ} \mathrm{C}$. The resulting mixture was stirred at that temperature for $30 \mathrm{~min}$ before aldehyde $6 \mathbf{b}$ (4.22 g, $9.24 \mathrm{mmol}$ ) was added as powder. The mixture was stirred at $-72{ }^{\circ} \mathrm{C}$ for $1 \mathrm{~h}$ and then warm to room temperature for $30 \mathrm{~min}$ before it was quenched with trifluoroacetic acid (3.00 mL, $40.8 \mathrm{mmol})$.

To the stirred solution of resulting mixture were added water $(9.00 \mathrm{~mL})$ and trifluoroacetic acid $(3.00 \mathrm{~mL})$ at room temperature. The mixture was stirred at that temperature for $12 \mathrm{~h}$ and then diluted with water. The organic volatiles were removed under vacuum and the residue was extracted with EtOAc three times. The combined organic phase was washed with saturated aq. $\mathrm{NaHCO}_{3}$ and brine, dried over anhydrous $\mathrm{Na}_{2} \mathrm{SO}_{4}$, filtered, and concentrated under vacuum. The residue was purified by silica gel chromatography (petroleum ether/acetone, 9:1 to 6:1) to afford compound $\mathbf{7 b}$ as a white solid $(3.14 \mathrm{~g}, 55 \%$ yield $):{ }^{1} \mathrm{H} \mathrm{NMR}\left(500 \mathrm{MHz}, \mathrm{CDCl}_{3}\right) \delta 7.64$ $(\mathrm{d}, J=8.1 \mathrm{~Hz}, 4 \mathrm{H}), 7.52(\mathrm{~d}, J=8.1 \mathrm{~Hz}, 4 \mathrm{H}), 7.33-7.29(\mathrm{~m}, 2 \mathrm{H}), 6.93-6.88(\mathrm{~m}, 3 \mathrm{H}), 6.64-6.62$ $(\mathrm{m}, 1 \mathrm{H}), 6.57(\mathrm{t}, J=2.2 \mathrm{~Hz}, 1 \mathrm{H}), 6.54(\mathrm{~d}, J=2.2 \mathrm{~Hz}, 2 \mathrm{H}), 6.15(\mathrm{~s}, 1 \mathrm{H}), 5.10-5.02(\mathrm{~m}, 4 \mathrm{H})$, $4.97(\mathrm{~s}, 2 \mathrm{H}), 3.81(\mathrm{~s}, 3 \mathrm{H}), 3.17-3.07(\mathrm{~m}, 1 \mathrm{H}), 3.04-2.97(\mathrm{~m}, 1 \mathrm{H}), 1.78-1.66(\mathrm{~m}, 2 \mathrm{H}), 1.01(\mathrm{t}$, $J=7.4 \mathrm{~Hz}, 3 \mathrm{H}) ;{ }^{13} \mathrm{C} \mathrm{NMR}\left(126 \mathrm{MHz}, \mathrm{CDCl}_{3}\right) \delta 170.1,163.8,160.1,159.9,152.9,146.6,140.6$, 139.9, 130.4 (q, $J=32.5 \mathrm{~Hz}), 129.6,127.8,127.6,125.7$ (q, $J=3.8 \mathrm{~Hz}), 124.2$ (q, $J=272.1$ Hz), 117.5, 115.1, 114.2, 106.1, 105.5, 102.4, 80.9, 70.4, 69.5, 55.4, 33.1, 24.0, 14.0; HRMS (ESI-TOF) $\mathrm{m} / \mathrm{z}[\mathrm{M}+\mathrm{Na}]^{+}$calcd for $\mathrm{C}_{41} \mathrm{H}_{34} \mathrm{~F}_{6} \mathrm{O}_{6} \mathrm{Na}^{+}$759.2152, found 759.2158. 

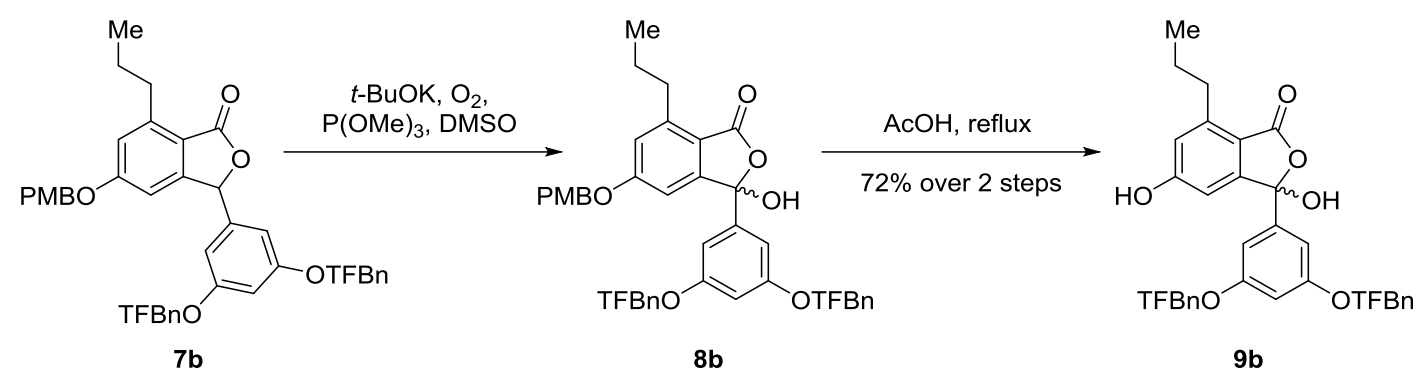

$8 b$

$9 b$

Compound 9b. To a stirred suspension of $7 \mathbf{b}(7.00 \mathrm{~g}, 9.50 \mathrm{mmol})$ and trimethyl phosphite (4.60 $\mathrm{mL}, 19.0 \mathrm{mmol})$ in DMSO $(20 \mathrm{~mL})$ was added a solution of potassium tert-butoxide (1.60 g, $1.50 \mathrm{mmol}$, dissolved in $20 \mathrm{~mL}$ DMSO) dropwise under oxygen at $20{ }^{\circ} \mathrm{C}$ cooling by water bath. The resulting red solution was stirred at that temperature until TLC indicated disappearance of the starting material. The colorless solution was quenched with aq. $\mathrm{HCl}(1.0$ M) and extracted with EtOAc three times. The combined organic phase was washed with saturated aq. $\mathrm{NaHCO}_{3}$ and brine, dried over anhydrous $\mathrm{Na}_{2} \mathrm{SO}_{4}$, filtered, and concentrated under vacuum to give crude $\mathbf{8 b}$ as a white solid. The crude $\mathbf{8 b}$ was used directly in the next step without further purification.

The crude $\mathbf{8 b}$ was dissolved in acetic acid $(500 \mathrm{~mL})$ and refluxed for $3 \mathrm{~h}$. The resulting mixture was concentrated under vacuum. The residue was dissolved in diethyl ether, washed with saturated aq. $\mathrm{NaHCO}_{3}$ and brine, dried over anhydrous $\mathrm{Na}_{2} \mathrm{SO}_{4}$, and filtered. Then chloroform was added to the mixture and diethyl ether was removed under vacuum. The resulting suspension was filtrated, and the filter cake was washed with chloroform and dried under vacuum to give $\mathbf{9 b}$ as a white solid $(4.31 \mathrm{~g}, 72 \%$ yield).

Compound 8b: ${ }^{1} \mathrm{H}$ NMR $\left(500 \mathrm{MHz}, \mathrm{CDCl}_{3}\right) \delta 7.63(\mathrm{~d}, J=8.1 \mathrm{~Hz}, 4 \mathrm{H}), 7.52(\mathrm{~d}, J=8.1$ $\mathrm{Hz}, 4 \mathrm{H}), 7.33-7.28(\mathrm{~m}, 2 \mathrm{H}), 6.92-6.87(\mathrm{~m}, 3 \mathrm{H}), 6.83(\mathrm{~d}, J=2.2 \mathrm{~Hz}, 2 \mathrm{H}), 6.80(\mathrm{~d}, J=2.1 \mathrm{~Hz}$, $1 \mathrm{H}), 6.57(\mathrm{t}, J=2.3 \mathrm{~Hz}, 1 \mathrm{H}), 5.11-5.03(\mathrm{~m}, 4 \mathrm{H}), 4.98(\mathrm{~s}, 2 \mathrm{H}), 4.06(\mathrm{~s}, 1 \mathrm{H}), 3.80(\mathrm{~s}, 3 \mathrm{H}), 3.05-$ $2.91(\mathrm{~m}, 2 \mathrm{H}), 1.72-1.61(\mathrm{~m}, 2 \mathrm{H}), 0.98(\mathrm{t}, J=7.3 \mathrm{~Hz}, 3 \mathrm{H}) ;{ }^{13} \mathrm{C} \mathrm{NMR}\left(126 \mathrm{MHz}, \mathrm{CDCl}_{3}\right) \delta 168.2$, 164.2, 160.0, 159.9, 153.0, 146.7, 141.8, 140.6, $130.4(\mathrm{q}, J=32.7 \mathrm{~Hz}), 129.7,127.7,127.6$, $125.7(\mathrm{q}, J=3.8 \mathrm{~Hz}), 124.2(\mathrm{q}, J=272.5 \mathrm{~Hz}), 118.5,115.0,114.3,106.1,105.2,103.5,102.8$, 70.6, 69.5, 55.4, 33.3, 23.9, 14.0; HRMS (ESI-TOF) $\mathrm{m} / \mathrm{z}[\mathrm{M}+\mathrm{Na}]^{+}$calcd for $\mathrm{C}_{41} \mathrm{H}_{34} \mathrm{~F}_{6} \mathrm{O}_{7} \mathrm{Na}^{+}$ 775.2101, found 775.2095.

Compound 9b: ${ }^{1} \mathrm{H}$ NMR (500 MHz, Acetone- $\left.d_{6}\right) \delta 7.74(\mathrm{~d}, J=8.3 \mathrm{~Hz}, 4 \mathrm{H}), 7.70(\mathrm{~d}, J=$ 
$8.3 \mathrm{~Hz}, 4 \mathrm{H}), 6.87(\mathrm{~d}, J=2.0 \mathrm{~Hz}, 2 \mathrm{H}), 6.85(\mathrm{~d}, J=1.8 \mathrm{~Hz}, 1 \mathrm{H}), 6.76-6.72(\mathrm{~m}, 2 \mathrm{H}), 5.25(\mathrm{~s}, 4 \mathrm{H})$, 2.97 (brs, 2H), 1.72-1.61 (m, 2H), 0.96 (t, $J=7.3 \mathrm{~Hz}, 3 \mathrm{H}) ;{ }^{13} \mathrm{C}$ NMR (126 MHz, acetone- $\left.d_{6}\right)$ $\delta 168.6,163.8,160.7,155.1,146.6,143.9,142.8,130.2(\mathrm{q}, J=32.2 \mathrm{~Hz}), 128.8,126.2(\mathrm{q}, J=$ $3.9 \mathrm{~Hz}), 125.3(\mathrm{q}, J=271.3 \mathrm{~Hz}), 119.2,115.1,108.0,106.1,104.4,102.8,69.8,33.6,24.7$, 14.1; HRMS (ESI-TOF) m/z [M + Na] ${ }^{+}$calcd for $\mathrm{C}_{33} \mathrm{H}_{26} \mathrm{~F}_{6} \mathrm{O}_{6} \mathrm{Na}^{+} 655.1526$, found 655.1520 .

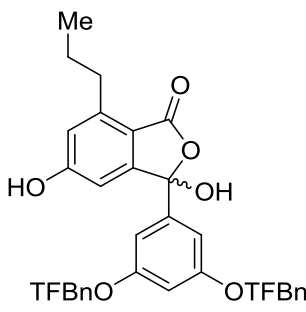

$9 b$

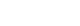

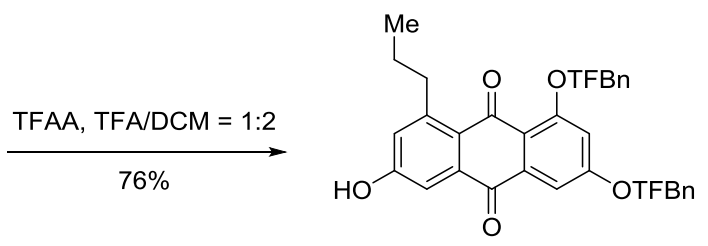

$10 \mathrm{~b}$

Compound 10b. To a stirred suspension of $9 \mathrm{~b}(3.16 \mathrm{~g}, 5.00 \mathrm{mmol})$ in $\mathrm{CH}_{2} \mathrm{Cl}_{2}(100 \mathrm{~mL})$ were added trifluoroacetic anhydride $(10.0 \mathrm{~mL}, 75.0 \mathrm{mmol})$ and trifluoroacetic acid $(50 \mathrm{~mL})$ under argon at $0{ }^{\circ} \mathrm{C}$. The mixture was stirred at that temperature for $4 \mathrm{~h}$. The resulting red solution was diluted with acetonitrile $(200 \mathrm{~mL})$ and concentrated under vacuum. The residue was dissolved in diethyl ether, washed with saturated aq. $\mathrm{NaHCO}_{3}$ and brine, dried over anhydrous $\mathrm{Na}_{2} \mathrm{SO}_{4}$, filtered, and concentrated under vacuum. The residue was recrystallized from diethyl ether give 10b as a yellow solid (2.32 g, 76\% yield): ${ }^{1} \mathrm{H}$ NMR $\left(500 \mathrm{MHz}, \mathrm{DMSO}-d_{6}\right) \delta 7.82$ $(\mathrm{d}, J=8.0 \mathrm{~Hz}, 2 \mathrm{H}), 7.77-7.70(\mathrm{~m}, 4 \mathrm{H}), 7.66(\mathrm{~d}, J=8.0 \mathrm{~Hz}, 2 \mathrm{H}), 7.32(\mathrm{~d}, J=2.1 \mathrm{~Hz}, 1 \mathrm{H}), 7.22$ $(\mathrm{d}, J=2.3 \mathrm{~Hz}, 1 \mathrm{H}), 7.11(\mathrm{~d}, J=2.3 \mathrm{~Hz}, 1 \mathrm{H}), 6.95(\mathrm{~d}, J=2.7 \mathrm{~Hz}, 1 \mathrm{H}), 5.36(\mathrm{~s}, 2 \mathrm{H}), 5.34(\mathrm{~s}$, 2H), 3.04-2.97 (m, 2H), 1.62-1.53 (m, 2H), $0.96(\mathrm{t}, J=7.3 \mathrm{~Hz}, 3 \mathrm{H}) ;{ }^{13} \mathrm{C}$ NMR $(126 \mathrm{MHz}$, DMSO- $\left.d_{6}\right) \delta 183.2,182.1,161.9,160.6,159.8,147.6,141.8,141.0,135.8,135.6,128.6(\mathrm{q}, J$ $=31.8 \mathrm{~Hz}), 128.1(\mathrm{q}, J=31.6 \mathrm{~Hz}), 128.1,127.1,125.4(\mathrm{q}, J=3.7 \mathrm{~Hz}), 125.1(\mathrm{q}, J=3.8 \mathrm{~Hz})$, 124.9, 124.3 (q, $J=271.6 \mathrm{~Hz}), 124.2$ (q, $J=272.1 \mathrm{~Hz}), 124.1,118.0,110.9,107.0,103.5,69.3$, 68.9, 36.6, 24.0, 14.2; HRMS (ESI-TOF) $\mathrm{m} / \mathrm{z}[\mathrm{M}+\mathrm{Na}]^{+}$calcd for $\mathrm{C}_{33} \mathrm{H}_{24} \mathrm{~F}_{6} \mathrm{O}_{5} \mathrm{Na}^{+}$637.1420, found 637.1425. 


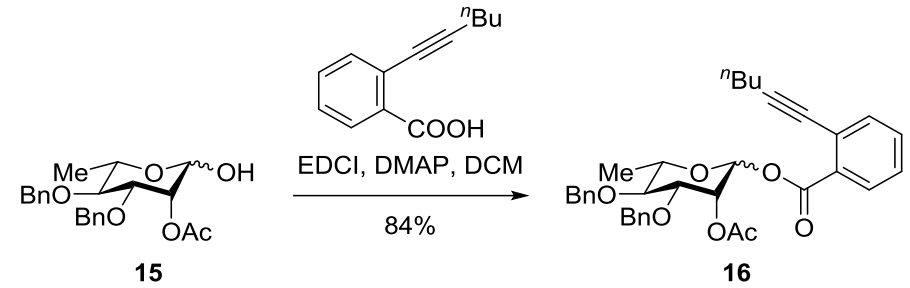

Compound 16. To a stirred solution of $\mathbf{1 5}^{\mathrm{S} 1}$ (309 $\left.\mathrm{mg}, 0.80 \mathrm{mmol}\right)$ in $\mathrm{CH}_{2} \mathrm{Cl}_{2}(5.0 \mathrm{~mL})$ were sequentially added 2-(1-hexyn-1-yl)benzoic acid ${ }^{\mathrm{S} 2}(220 \mathrm{mg}, 1.10 \mathrm{mmol})$, 4-dimethylaminopyridine (11.0 $\mathrm{mg}, \quad 0.09 \mathrm{mmol})$, and ethyl-3-(3-dimethylaminopropyl)carbodiimide hydrochloride (260 mg, $1.35 \mathrm{mmol})$ at room temperature. The mixture was stirred at that temperature for $8 \mathrm{~h}$ before it was quenched with ethanol. The organic volatiles were removed under vacuum and the residue was purified by silica gel chromatography (petroleum ether/EtOAc, 10:1) to afford $16(\alpha / \beta=1: 1)$ as a colorless syrup (381 mg, 84\% yield): ${ }^{1} \mathrm{H}$ NMR $\left(500 \mathrm{MHz}, \mathrm{CDCl}_{3}\right)$ selected signals for the $\alpha$ anomer $\delta 6.29(\mathrm{~d}, J=2.0 \mathrm{~Hz}, 1 \mathrm{H}), 5.51(\mathrm{dd}, J=$ 3.4, 2.0 Hz, 1H), 4.09 (dd, $J=9.4,3.4 \mathrm{~Hz}, 1 \mathrm{H}), 4.01(\mathrm{dq}, J=9.6,6.2 \mathrm{~Hz}, 1 \mathrm{H}), 1.37$ (d, $J=6.2$ $\mathrm{Hz}, 2 \mathrm{H})$; selected signals for the $\beta$ anomer $\delta 5.97(\mathrm{~d}, J=1.2 \mathrm{~Hz}, 1 \mathrm{H}), 5.75(\mathrm{dd}, J=3.2,1.2 \mathrm{~Hz}$, $1 \mathrm{H}), 3.77(\mathrm{dd}, J=9.2,3.3 \mathrm{~Hz}, 1 \mathrm{H}), 3.61(\mathrm{dq}, J=9.4,6.1 \mathrm{~Hz}, 1 \mathrm{H}), 1.41(\mathrm{~d}, J=6.1 \mathrm{~Hz}, 2 \mathrm{H}) ;{ }^{13} \mathrm{C}$ NMR (126 MHz, $\mathrm{CDCl}_{3}$ ) selected signals for the $\alpha$ anomer $\delta 92.1,68.04,77.7,70.6,18.2$; selected signals for the $\beta$ anomer $\delta$ 91.6, 67.97, 80.0, 72.9, 18.1; HRMS (ESI-TOF) $\mathrm{m} / \mathrm{z}$ [M + $\mathrm{Na}]^{+}$calcd for $\mathrm{C}_{35} \mathrm{H}_{38} \mathrm{O}_{7} \mathrm{Na}^{+}$593.2510, found 593.2508.

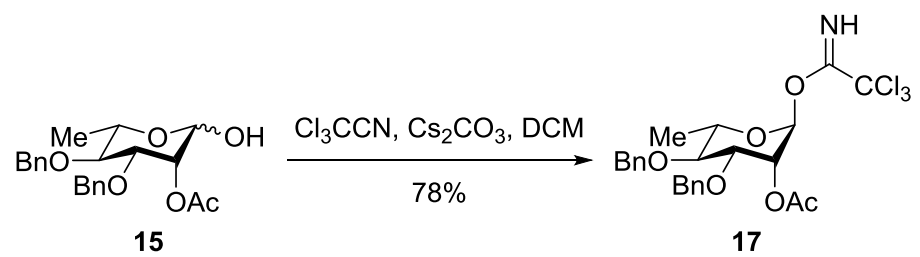

Compound 17. ${ }^{\mathrm{S} 3}$ To a stirred solution of $\mathbf{1 5}^{\mathrm{S} 1}(32.0 \mathrm{mg}, 0.08 \mathrm{mmol})$ in $\mathrm{CH}_{2} \mathrm{Cl}_{2}(1.0 \mathrm{~mL})$ were added trichloroacetonitrile $(83.0 \mu \mathrm{L}, 0.83 \mathrm{mmol})$ and $\mathrm{Cs}_{2} \mathrm{CO}_{3}(40.0 \mathrm{mg}, 0.12 \mathrm{mmol})$ at room temperature. The mixture was stirred at that temperature for $1 \mathrm{~h}$. The organic volatiles were removed under vacuum and the residue was purified by silica gel chromatography (petroleum ether/EtOAc, 5:1) to afford 17 ( $\alpha$ only) as a colorless syrup (34.0 mg, $78 \%$ yield): ${ }^{1} \mathrm{H}$ NMR $\left(500 \mathrm{MHz}, \mathrm{CDCl}_{3}\right) \delta 8.66(\mathrm{~s}, 1 \mathrm{H}), 7.38-7.27(\mathrm{~m}, 10 \mathrm{H}), 6.18(\mathrm{~d}, J=2.0 \mathrm{~Hz}, 1 \mathrm{H}), 5.49(\mathrm{dd}, J=$ 
3.4, 2.0 Hz, 1H), $4.94(\mathrm{~d}, J=10.8 \mathrm{~Hz}, 1 \mathrm{H}), 4.74(\mathrm{~d}, J=11.2 \mathrm{~Hz}, 1 \mathrm{H}), 4.64(\mathrm{~d}, J=10.7 \mathrm{~Hz}, 1 \mathrm{H})$, $4.58(\mathrm{~d}, J=11.2 \mathrm{~Hz}, 1 \mathrm{H}), 4.00(\mathrm{dd}, J=9.5,3.4 \mathrm{~Hz}, 1 \mathrm{H}), 3.98-3.91(\mathrm{~m}, 1 \mathrm{H}), 3.54(\mathrm{t}, J=9.6$ $\mathrm{Hz}, 1 \mathrm{H}), 2.20(\mathrm{~s}, 3 \mathrm{H}), 1.36(\mathrm{~d}, J=6.2 \mathrm{~Hz}, 3 \mathrm{H}) ;{ }^{13} \mathrm{C} \mathrm{NMR}\left(126 \mathrm{MHz}, \mathrm{CDCl}_{3}\right) \delta 170.2,160.2$, 138.2, 137.6, 128.6, 128.6, 128.5, 128.3, 128.1, 128.0, 95.3, 91.0, 79.5, 77.3, 75.8, 72.1, 70.8, 67.7, 21.1, 18.1; HRMS (ESI-TOF) $\mathrm{m} / \mathrm{z}[\mathrm{M}+\mathrm{Na}]^{+}$calcd for $\mathrm{C}_{24} \mathrm{H}_{26} \mathrm{Cl}_{3} \mathrm{NO}_{6} \mathrm{Na}^{+}$552.0718, found 552.0719.

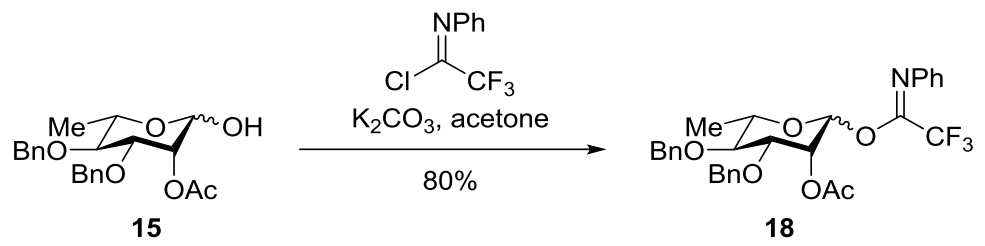

Compound 18. ${ }^{\mathrm{S} 4}$ To a stirred solution of $15^{\mathrm{S} 1}(130.0 \mathrm{mg}, 0.34 \mathrm{mmol})$ in acetone $(3.0 \mathrm{~mL})$ were added $N$-phenyl-trifluoroacetimidoyl chloride $(67 \mu \mathrm{L}, 0.40 \mathrm{mmol})$ and $\mathrm{K}_{2} \mathrm{CO}_{3}(186 \mathrm{mg}, 1.35$ mmol) at room temperature. The mixture was stirred at that temperature for $1 \mathrm{~h}$. The organic volatiles were removed under vacuum and the residue was purified by silica gel chromatography (petroleum ether/EtOAc, 10:1) to afford $\mathbf{1 8}$ as a colorless syrup (150 mg, 80\% yield): ${ }^{1} \mathrm{H}$ NMR $\left(500 \mathrm{MHz}, \mathrm{CDCl}_{3}\right)$ selected signals for the major anomer $\delta 7.38-7.28(\mathrm{~m}$, 15H), 7.13-7.08 (m, 1H), $6.83(\mathrm{~d}, J=7.8 \mathrm{~Hz}, 2 \mathrm{H}), 6.15(\mathrm{brs}, 1 \mathrm{H}), 5.48(\mathrm{t}, J=2.7 \mathrm{~Hz}, 1 \mathrm{H}), 4.94$ $(\mathrm{d}, J=10.7 \mathrm{~Hz}, 1 \mathrm{H}), 4.74(\mathrm{~d}, J=11.1 \mathrm{~Hz}, 1 \mathrm{H}), 4.67-4.58(\mathrm{~m}, 2 \mathrm{H}), 3.99(\mathrm{dd}, J=9.5,3.4 \mathrm{~Hz}$, 1H), $3.90(\mathrm{dq}, J=12.4,6.4 \mathrm{~Hz}, 1 \mathrm{H}), 3.52(\mathrm{t}, J=9.5 \mathrm{~Hz}, 1 \mathrm{H}), 2.17(\mathrm{~s}, 3 \mathrm{H}), 1.38(\mathrm{~d}, J=6.2 \mathrm{~Hz}$, $3 \mathrm{H}) ;{ }^{13} \mathrm{C} \mathrm{NMR}\left(126 \mathrm{MHz}, \mathrm{CDCl}_{3}\right.$ ) selected signals for the major anomer $\delta 170.2,143.4,138.2$, 137.7, 128.9, 128.6, 128.6, 128.4, 128.2, 128.1, 128.0, 124.6, 119.5, 94.3, 79.4, 77.5, 75.8, 72.4, 70.6, 67.7, 21.1, 18.1; HRMS (ESI-TOF) m/z $[\mathrm{M}+\mathrm{Na}]^{+}$calcd for $\mathrm{C}_{30} \mathrm{H}_{30} \mathrm{~F}_{3} \mathrm{NO}_{6} \mathrm{Na}^{+}$580.1917, found 580.1919.

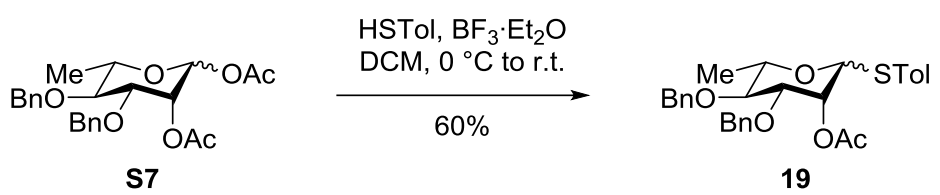

Compound 19. To a stirred solution of $\mathbf{S} 7^{\mathrm{S} 5}(5.35 \mathrm{~g}, 12.5 \mathrm{mmol})$ and $p$-thiocresol $(3.10 \mathrm{~g}, 25.0$ 
mmol $)$ in $\mathrm{CH}_{2} \mathrm{Cl}_{2}(100 \mathrm{~mL})$ was added $\mathrm{BF}_{3} \cdot \mathrm{Et}_{2} \mathrm{O}(3.10 \mathrm{~mL}, 25.0 \mathrm{mmol})$ at $0{ }^{\circ} \mathrm{C}$ under argon. The mixture was warm to room temperature for $16 \mathrm{~h}$ before it was quenched with aq. $\mathrm{NaOH}$ $(1.0 \mathrm{M})$. The mixture was extracted with EtOAc three times. The combined organic phase was washed with aq. $\mathrm{NaOH}(1.0 \mathrm{M})$, aq. $\mathrm{HCl}(1.0 \mathrm{M})$, saturated aq. $\mathrm{NaHCO}_{3}$, and brine sequentially, and was then dried over anhydrous $\mathrm{Na}_{2} \mathrm{SO}_{4}$, filtered, and concentrated under vacuum. The residue was purified by silica gel chromatography (petroleum ether/EtOAc, 20:1 to 10:1) to afford $19(\alpha / \beta=2: 1)$ as a colorless syrup $(3.71 \mathrm{~g}, 60 \%$ yield $):{ }^{1} \mathrm{H}$ NMR $\left(500 \mathrm{MHz}, \mathrm{CDCl}_{3}\right)$ selected signals for the $\alpha$ anomer $\delta 5.60(\mathrm{dd}, J=3.3,1.7 \mathrm{~Hz}, 1 \mathrm{H}), 5.33(\mathrm{~d}, J=1.6 \mathrm{~Hz}, 1 \mathrm{H}), 4.94$ $(\mathrm{d}, J=10.8 \mathrm{~Hz}, 1 \mathrm{H}), 4.72(\mathrm{~d}, J=11.2 \mathrm{~Hz}, 1 \mathrm{H}), 4.63(\mathrm{~d}, J=10.8 \mathrm{~Hz}, 1 \mathrm{H}), 4.56(\mathrm{~d}, J=11.2 \mathrm{~Hz}$, $1 \mathrm{H}), 4.24(\mathrm{dq}, J=9.5,6.2 \mathrm{~Hz}, 1 \mathrm{H}), 3.92(\mathrm{dd}, J=9.3,3.3 \mathrm{~Hz}, 1 \mathrm{H}), 3.51(\mathrm{t}, J=9.4 \mathrm{~Hz}, 1 \mathrm{H}), 2.33$ $(\mathrm{s}, 3 \mathrm{H}), 2.14(\mathrm{~s}, 3 \mathrm{H}), 1.35(\mathrm{~d}, J=6.2 \mathrm{~Hz}, 3 \mathrm{H})$; selected signals for the $\beta$ anomer $\delta 5.79$ (dd, $J=$ $3.5,1.2 \mathrm{~Hz}, 1 \mathrm{H}), 4.90(\mathrm{~d}, J=10.8 \mathrm{~Hz}, 1 \mathrm{H}), 4.79(\mathrm{~d}, J=11.1 \mathrm{~Hz}, 1 \mathrm{H}), 4.75(\mathrm{~d}, J=1.2 \mathrm{~Hz}, 1 \mathrm{H})$, $4.62(\mathrm{~d}, J=10.8 \mathrm{~Hz}, 1 \mathrm{H}), 4.49(\mathrm{~d}, J=11.1 \mathrm{~Hz}, 1 \mathrm{H}), 3.62(\mathrm{dd}, J=9.1,3.5 \mathrm{~Hz}, 1 \mathrm{H}), 3.46(\mathrm{t}, J=$ $9.2 \mathrm{~Hz}, 1 \mathrm{H}), 3.38(\mathrm{dq}, J=9.2,6.0 \mathrm{~Hz}, 1 \mathrm{H}), 2.34(\mathrm{~s}, 3 \mathrm{H}), 2.24(\mathrm{~s}, 3 \mathrm{H}), 1.40(\mathrm{~d}, J=6.0 \mathrm{~Hz}, 3 \mathrm{H})$; ${ }^{13} \mathrm{C}$ NMR $\left(126 \mathrm{MHz}, \mathrm{CDCl}_{3}\right.$ ) selected signals for the $\alpha$ anomer $\delta 170.4,138.5,138.0,137.8$, $132.45,130.19,130.0,128.59,128.54,128.3,128.1,128.00,127.89,86.6,80.3,78.4,75.63$, 72.0, 70.7, 69.1, 21.26, 21.25, 18.0; selected signals for the $\beta$ anomer $\delta 170.6,138.4,138.2$, $137.7,132.40,130.24,129.9,128.57,128.53,128.4,128.2,127.98,127.93,85.8,81.3,79.4$, 76.3, 75.65, 71.9, 70.5, 21.27, 21.1, 18.4; HRMS (ESI-TOF) $\mathrm{m} / \mathrm{z}[\mathrm{M}+\mathrm{Na}]^{+}$calcd for $\mathrm{C}_{29} \mathrm{H}_{32} \mathrm{O}_{5} \mathrm{SNa}^{+}$515.1863, found 515.1862.

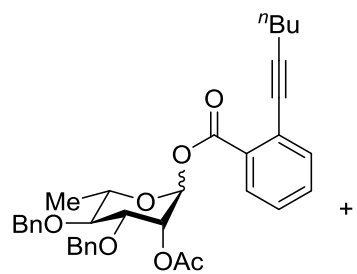

16

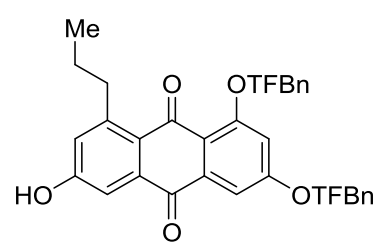

$10 \mathrm{~b}$

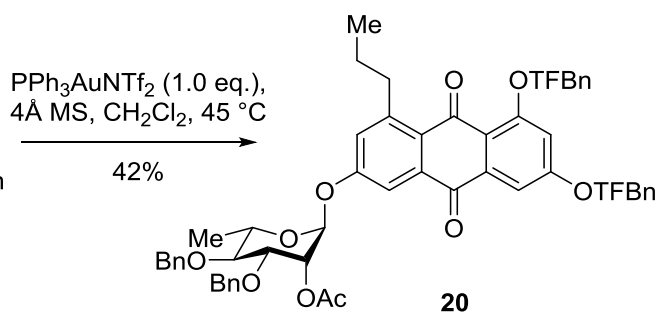

20

Compound 20. To a stirred solution of glycosyl donor 16 (25.0 mg, $0.044 \mathrm{mmol})$ and acceptor 10b (9.0 mg, $0.015 \mathrm{mmol})$ in $\mathrm{CH}_{2} \mathrm{Cl}_{2}(2.5 \mathrm{~mL})$ was added activated $4 \AA$ molecular sieves (150 $\mathrm{mg}$ ) at room temperature under argon. The mixture was stirred at that temperature for $1 \mathrm{~h}$ before 
it was added $\mathrm{Ph}_{3} \mathrm{PAuNTf}_{2}(10.8 \mathrm{mg}, 0.015 \mathrm{mmol})$. The resulting mixture was heated with oil bath to $45^{\circ} \mathrm{C}$ and stirred for $24 \mathrm{~h}$ before it was quenched with triphenylphosphine resin $(30.0$ $\mathrm{mg}, \sim 1.0 \mathrm{mmol} / \mathrm{g}, 0.030 \mathrm{mmol})$. The mixture was filtered through a pad of celite and washed with $\mathrm{CH}_{2} \mathrm{Cl}_{2}$ three times. The filtrate was concentrated under vacuum. The residue was purified by silica gel chromatography (petroleum ether/EtOAc, 5:1) to afford 20 as a yellow foam (7.1 $\mathrm{mg}, 42 \%$ yield).

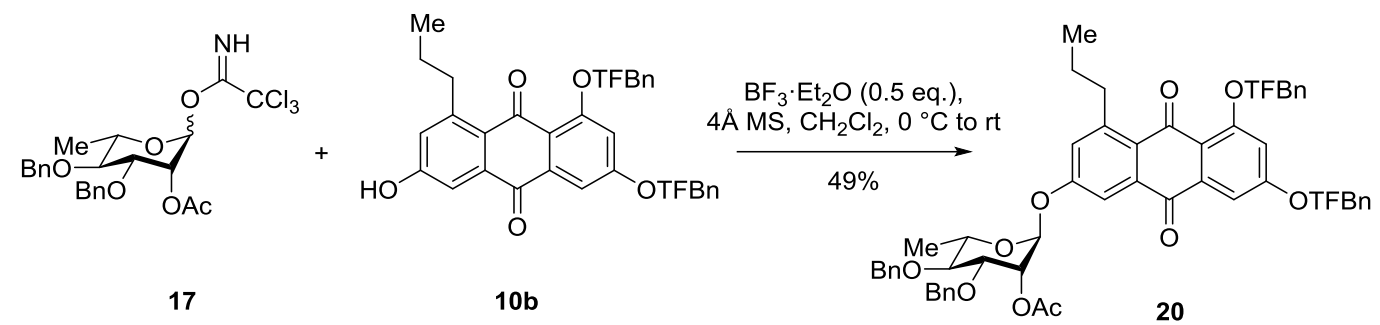

To a stirred solution of glycosyl donor $17(16.0 \mathrm{mg}, 0.030 \mathrm{mmol})$ and acceptor $\mathbf{1 0 b}(6.1$ $\mathrm{mg}, 0.010 \mathrm{mmol})$ in $\mathrm{CH}_{2} \mathrm{Cl}_{2}(2.0 \mathrm{~mL})$ was added activated $4 \AA$ molecular sieves $(90 \mathrm{mg})$ at room temperature under argon. The mixture was stirred at that temperature for $1 \mathrm{~h}$ before it was cooled to $0{ }^{\circ} \mathrm{C}$. To the resulting mixture was added $\mathrm{BF}_{3} \cdot \mathrm{Et}_{2} \mathrm{O}(0.6 \mu \mathrm{L}, 0.005 \mathrm{mmol})$ at $0{ }^{\circ} \mathrm{C}$. The mixture was stirred at $0{ }^{\circ} \mathrm{C}$ for $2 \mathrm{~h}$ and then warm to room temperature for $30 \mathrm{~min}$ before it was quenched with $\mathrm{Et}_{3} \mathrm{~N}$. The mixture was filtered through a pad of celite and washed with $\mathrm{CH}_{2} \mathrm{Cl}_{2}$ three times. The filtrate was concentrated under vacuum. The residue was purified by silica gel chromatography (petroleum ether/EtOAc, 10:1) to afford 20 as a yellow foam (4.8 mg, 49\%).

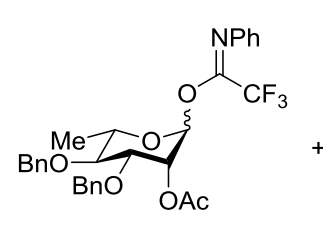

18

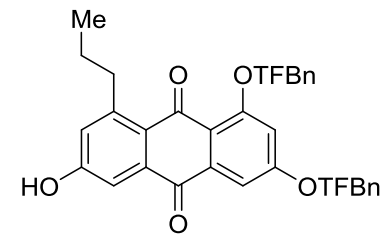

$10 \mathrm{~b}$

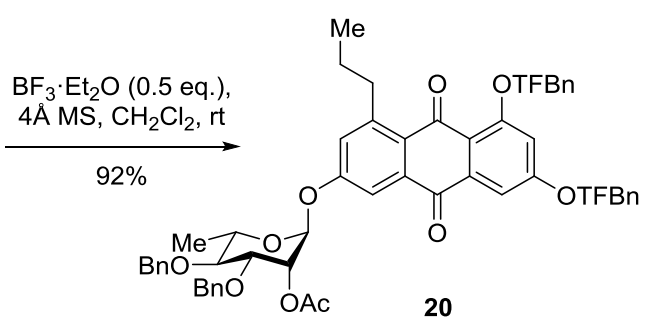

20

To a stirred solution of glycosyl donor $18(56.0 \mathrm{mg}, 0.10 \mathrm{mmol})$ and acceptor $\mathbf{1 0 b}(20.4$ $\mathrm{mg}, 0.033 \mathrm{mmol})$ in $\mathrm{CH}_{2} \mathrm{Cl}_{2}(7.0 \mathrm{~mL})$ was added activated $4 \AA$ molecular sieves $(300 \mathrm{mg})$ at room temperature under argon. The mixture was stirred at that temperature for $1 \mathrm{~h}$, then added $\mathrm{BF}_{3} \cdot \mathrm{Et}_{2} \mathrm{O}(2.1 \mu \mathrm{L}, 0.017 \mathrm{mmol})$. The mixture was stirred at room temperature for $2 \mathrm{~h}$ before it was quenched with $\mathrm{Et}_{3} \mathrm{~N}$. The mixture was filtered through a pad of celite and washed with 
$\mathrm{CH}_{2} \mathrm{Cl}_{2}$ three times. The filtrate was concentrated under vacuum. The residue was purified by silica gel chromatography (petroleum ether/EtOAc, 10:1) to afford 20 as a yellow foam (30.0 $\mathrm{mg}, 92 \%$ yield).

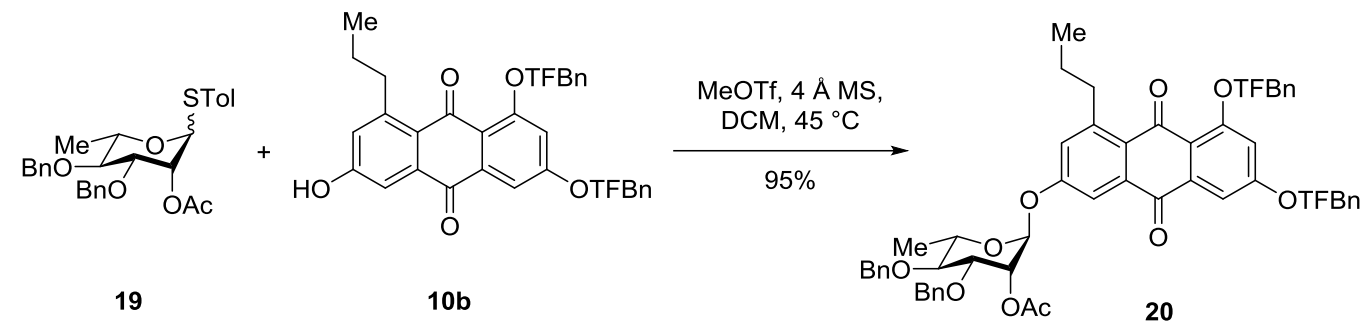

In a sealed flask, a solution of rhamnosyl donor $19(1.97 \mathrm{~g}, 4.00 \mathrm{mmol})$ and acceptor 10b $(1.23 \mathrm{~g}, 2.00 \mathrm{mmol})$ in $\mathrm{CH}_{2} \mathrm{Cl}_{2}(200 \mathrm{~mL})$ was added activated $4 \AA$ A molecular sieves $(10.0 \mathrm{~g})$ at room temperature under argon. The mixture was stirred at that temperature for $1 \mathrm{~h}$, then methyl triflate $(1.13 \mathrm{~mL}, 10.0 \mathrm{mmol})$ was added. The mixture was heated with oil bath to $45^{\circ} \mathrm{C}$ and stirred at that temperature for $36 \mathrm{~h}$ before it was quenched with a 1:1 mixture of methanol and $\mathrm{Et}_{3} \mathrm{~N}(5.00 \mathrm{~mL})$. The mixture was filtered through a pad of celite and washed with $\mathrm{CH}_{2} \mathrm{Cl}_{2}$ three times. The filtrate was concentrated under vacuum. The residue was purified by silica gel chromatography (petroleum ether/EtOAc, 10:1 to 4:1) to afford 20 as a yellow foam (1.86 g, $95 \%$ yield).

Compound 20: $[\alpha]_{\mathrm{D}}^{25}-63.5\left(c 0.8, \mathrm{CHCl}_{3}\right) ;{ }^{1} \mathrm{H} \mathrm{NMR}\left(500 \mathrm{MHz}, \mathrm{CDCl}_{3}\right) \delta$ 7.77-7.72 (m, 3H), $7.68(\mathrm{~d}, J=8.3 \mathrm{~Hz}, 4 \mathrm{H}), 7.56(\mathrm{~d}, J=8.1 \mathrm{~Hz}, 2 \mathrm{H}), 7.44(\mathrm{~d}, J=2.4 \mathrm{~Hz}, 1 \mathrm{H}), 7.41-7.28(\mathrm{~m}$, $10 \mathrm{H}), 7.17(\mathrm{~d}, J=2.7 \mathrm{~Hz}, 1 \mathrm{H}), 6.86(\mathrm{~d}, J=2.4 \mathrm{~Hz}, 1 \mathrm{H}), 5.67(\mathrm{~d}, J=1.7 \mathrm{~Hz}, 1 \mathrm{H}), 5.57$ (dd, $J=$ 3.4, 1.9 Hz, 1H), $5.30(\mathrm{~s}, 2 \mathrm{H}), 5.24(\mathrm{~s}, 2 \mathrm{H}), 4.97(\mathrm{~d}, J=10.8 \mathrm{~Hz}, 1 \mathrm{H}), 4.79(\mathrm{~d}, J=11.2 \mathrm{~Hz}, 1 \mathrm{H})$, $4.66(\mathrm{~d}, J=10.8 \mathrm{~Hz}, 1 \mathrm{H}), 4.65(\mathrm{~d}, J=11.2 \mathrm{~Hz}, 1 \mathrm{H}), 4.17(\mathrm{dd}, J=9.3,3.4 \mathrm{~Hz}, 1 \mathrm{H}), 3.81(\mathrm{dt}, J$ $=9.5,6.2 \mathrm{~Hz}, 1 \mathrm{H}), 3.56(\mathrm{t}, J=9.4 \mathrm{~Hz}, 1 \mathrm{H}), 3.24-3.13(\mathrm{~m}, 2 \mathrm{H}), 2.23(\mathrm{~s}, 3 \mathrm{H}), 1.78-1.69(\mathrm{~m}$, $2 \mathrm{H}), 1.32(\mathrm{~d}, J=6.2 \mathrm{~Hz}, 3 \mathrm{H}), 1.08(\mathrm{t}, J=7.3 \mathrm{~Hz}, 3 \mathrm{H}) ;{ }^{13} \mathrm{C} \mathrm{NMR}\left(126 \mathrm{MHz}, \mathrm{CDCl}_{3}\right) \delta 183.7$, $183.1,170.5,162.4,160.3,158.1,148.8,140.5,139.8,138.3,137.9,136.8,136.1,130.7$ (q, $J$ $=32.5 \mathrm{~Hz}), 130.1(\mathrm{q}, J=32.4 \mathrm{~Hz}), 128.6,128.5,128.2,128.2,128.1,128.0,127.9,127.7,126.8$, $125.8(\mathrm{q}, J=3.8 \mathrm{~Hz}), 125.7(\mathrm{q}, J=3.7 \mathrm{~Hz}), 125.1,124.2(\mathrm{q}, J=272.0 \mathrm{~Hz}), 124.1(\mathrm{q}, J=271.9$ Hz), 119.4, 111.9, 107.7, 103.5, 95.5, 79.8, 77.8, 75.7, 72.2, 70.3, 69.7, 69.1, 68.6, 37.4, 24.5, 
21.2, 18.1, 14.5; HRMS (ESI-TOF) $\mathrm{m} / \mathrm{z}[\mathrm{M}+\mathrm{Na}]^{+}$calcd for $\mathrm{C}_{55} \mathrm{H}_{48} \mathrm{~F}_{6} \mathrm{O}_{10} \mathrm{Na}^{+}$1005.3044, found 1005.3025 .
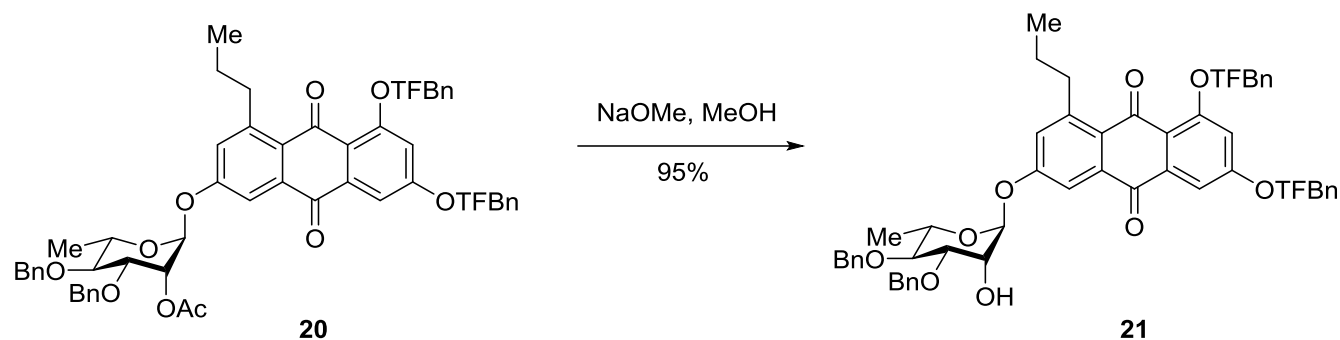

Compound 21. To a stirred suspension of 20 (1.68 g, $1.71 \mathrm{mmol})$ in methanol (17.0 mL) was added sodium methoxide $(18.5 \mathrm{mg}, 0.34 \mathrm{mmol})$ at room temperature under argon. The resulting mixture was stirred at that temperature for $2 \mathrm{~h}$ before it was quenched with aq. $\mathrm{HCl}(1.00 \mathrm{M}$, $0.34 \mathrm{~mL}, 0.34 \mathrm{mmol}$ ), and then concentrated under vacuum. The residue was purified by silica gel chromatography (petroleum ether/acetone, 3:1) to afford 21 as a yellow foam $(1.53 \mathrm{~g}, 95 \%$ yield): $[\alpha]_{\mathrm{D}}^{25}-94.6\left(c 0.5, \mathrm{CHCl}_{3}\right) ;{ }^{1} \mathrm{H} \mathrm{NMR}\left(500 \mathrm{MHz}, \mathrm{CDCl}_{3}\right) \delta 7.76(\mathrm{~d}, J=2.7 \mathrm{~Hz}, 1 \mathrm{H}), 7.74$ $(\mathrm{d}, J=8.1 \mathrm{~Hz}, 2 \mathrm{H}), 7.67(\mathrm{~d}, J=8.4 \mathrm{~Hz}, 4 \mathrm{H}), 7.56(\mathrm{~d}, J=8.0 \mathrm{~Hz}, 2 \mathrm{H}), 7.44-7.28(\mathrm{~m}, 11 \mathrm{H})$, $7.19(\mathrm{~d}, J=2.7 \mathrm{~Hz}, 1 \mathrm{H}), 6.85(\mathrm{~d}, J=2.4 \mathrm{~Hz}, 1 \mathrm{H}), 5.73$ (d, $J=1.4 \mathrm{~Hz}, 1 \mathrm{H}), 5.30$ (s, 2H), 5.23 $(\mathrm{s}, 2 \mathrm{H}), 4.92(\mathrm{~d}, J=10.8 \mathrm{~Hz}, 1 \mathrm{H}), 4.83-4.76(\mathrm{~m}, 2 \mathrm{H}), 4.68(\mathrm{~d}, J=10.9 \mathrm{~Hz}, 1 \mathrm{H}), 4.25(\mathrm{dd}, J=$ 3.3, $1.8 \mathrm{~Hz}, 1 \mathrm{H}), 4.07(\mathrm{dd}, J=9.1,3.4 \mathrm{~Hz}, 1 \mathrm{H}), 3.82-3.75(\mathrm{~m}, 1 \mathrm{H}), 3.57(\mathrm{t}, J=9.3 \mathrm{~Hz}, 1 \mathrm{H})$, 3.25-3.12 (m, 2H), 2.74 (brs, $1 \mathrm{H}), 1.78-1.69(\mathrm{~m}, 2 \mathrm{H}), 1.28(\mathrm{~d}, J=6.2 \mathrm{~Hz}, 3 \mathrm{H}), 1.07(\mathrm{t}, J=7.3$ $\mathrm{Hz}, 3 \mathrm{H}) ;{ }^{13} \mathrm{C} \mathrm{NMR}\left(126 \mathrm{MHz}, \mathrm{CDCl}_{3}\right) \delta 183.7,183.1,162.4,160.2,158.3,148.7,140.5,139.8$, 138.3, 137.8, 136.8, 136.1, $130.7(\mathrm{q}, J=32.6 \mathrm{~Hz}), 130.1(\mathrm{q}, J=32.3 \mathrm{~Hz}), 128.8,128.6,128.3$, 128.1, 128.0, 128.0, 127.7, 126.8, $125.8(\mathrm{q}, J=3.8 \mathrm{~Hz}), 125.7(\mathrm{q}, J=3.7 \mathrm{~Hz}), 125.0,124.2(\mathrm{q}$, $J=272.1 \mathrm{~Hz}), 124.1(\mathrm{q}, J=272.1 \mathrm{~Hz}), 119.4,112.1,107.7,103.4,96.9,79.8,79.7,75.7,72.5$, 70.3, 69.7, 68.7, 68.4, 37.5, 24.5, 18.1, 14.5; HRMS (ESI-TOF) $\mathrm{m} / \mathrm{z}[\mathrm{M}+\mathrm{Na}]^{+}$calcd for $\mathrm{C}_{53} \mathrm{H}_{46} \mathrm{~F}_{6} \mathrm{O}_{9} \mathrm{Na}^{+}$963.2938, found 963.2940. 

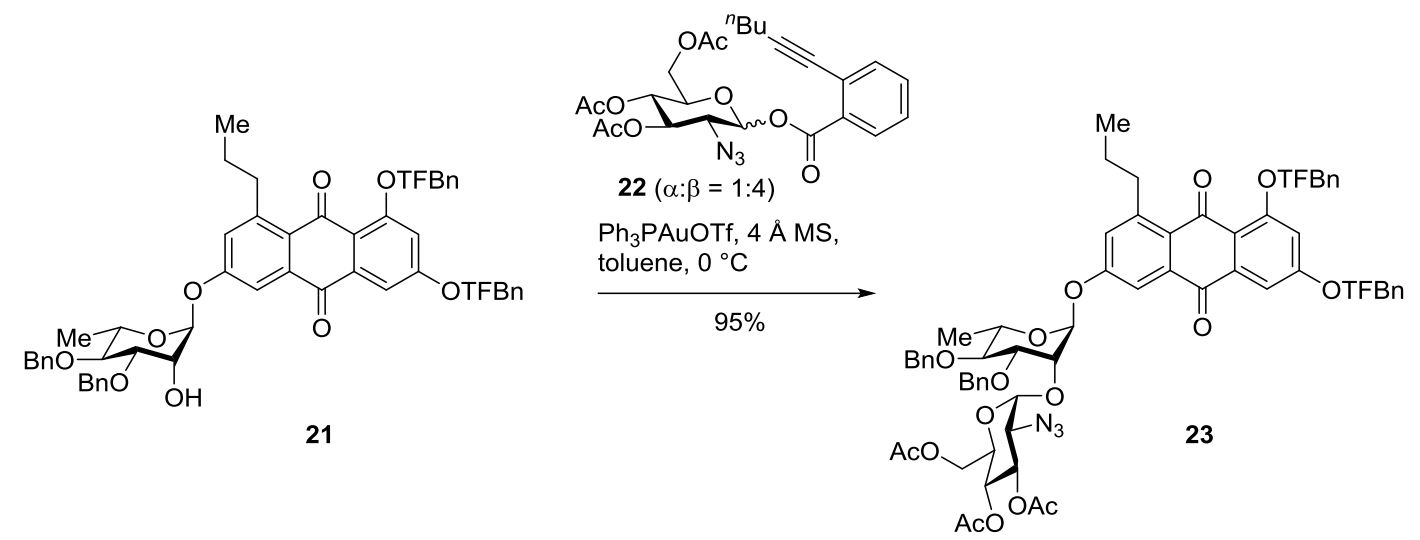

Compound 23. Preparation of $\mathrm{Ph}_{3} \mathrm{PAuOTf}$ solution: To a stirred suspension of $\mathrm{Ph}_{3} \mathrm{PAuCl}$ (26.3 $\mathrm{mg}, 0.053 \mathrm{mmol})$ in toluene $(2.0 \mathrm{~mL})$ was added AgOTf $(13.7 \mathrm{mg}, 0.053 \mathrm{mmol})$ at room temperature under argon. The mixture was stirred at that temperature for $15 \mathrm{~min}$. The resulting mixture was stood for 5 minutes, and then the supernatant was drawn with a syringe.

To a stirred solution of glycosyl donor $\mathbf{2 2}^{\mathrm{S} 6}(82.1 \mathrm{mg}, 0.16 \mathrm{mmol})$ and acceptor 21 (50.0 $\mathrm{mg}, 0.053 \mathrm{mmol})$ in toluene $(2.5 \mathrm{~mL})$ was added activated $4 \AA$ molecular sieves $(250 \mathrm{mg})$ at room temperature under argon. The mixture was stirred at that temperature for $1 \mathrm{~h}$ before it was cooled to $0{ }^{\circ} \mathrm{C}$. To the resulting mixture was added the $\mathrm{Ph}_{3} \mathrm{PAuOTf}$ solution $(0.4 \mathrm{~mL}, 0.011$ mmol) dropwise at $0{ }^{\circ} \mathrm{C}$. The mixture was stirred at that temperature for $12 \mathrm{~h}$ before it was quenched with triphenylphosphine resin $(21.0 \mathrm{mg}, \sim 1.0 \mathrm{mmol} / \mathrm{g}, 0.021 \mathrm{mmol})$. The mixture was filtered through a pad of celite and washed with $\mathrm{CH}_{2} \mathrm{Cl}_{2}$ three times. The filtrate was concentrated under vacuum. The residue was purified by silica gel chromatography (petroleum ether/acetone, 5:1 to 3:1) to afford 23 as a yellow foam $(63.0 \mathrm{mg}, 95 \%$ yield $)$ : $[\alpha]_{\mathrm{D}}^{25}+14.4(c$ $\left.0.8, \mathrm{CHCl}_{3}\right) ;{ }^{1} \mathrm{H} \mathrm{NMR}\left(600 \mathrm{MHz}, \mathrm{CDCl}_{3}\right) \delta 7.77(\mathrm{~d}, J=2.7 \mathrm{~Hz}, 1 \mathrm{H}), 7.74(\mathrm{~d}, J=8.1 \mathrm{~Hz}, 2 \mathrm{H})$, $7.68(\mathrm{~d}, J=8.9 \mathrm{~Hz}, 4 \mathrm{H}), 7.57(\mathrm{~d}, J=8.1 \mathrm{~Hz}, 2 \mathrm{H}), 7.44(\mathrm{~d}, J=2.4 \mathrm{~Hz}, 1 \mathrm{H}), 7.41-7.27(\mathrm{~m}, 10 \mathrm{H})$, $7.20(\mathrm{~d}, J=2.7 \mathrm{~Hz}, 1 \mathrm{H}), 6.87(\mathrm{~d}, J=2.4 \mathrm{~Hz}, 1 \mathrm{H}), 5.70(\mathrm{~d}, J=1.6 \mathrm{~Hz}, 1 \mathrm{H}), 5.60(\mathrm{dd}, J=10.6$, $9.3 \mathrm{~Hz}, 1 \mathrm{H}), 5.32(\mathrm{~s}, 2 \mathrm{H}), 5.26(\mathrm{~s}, 2 \mathrm{H}), 5.14(\mathrm{~d}, J=3.6 \mathrm{~Hz}, 1 \mathrm{H}), 5.09-5.04(\mathrm{~m}, 1 \mathrm{H}), 4.99(\mathrm{~d}, J$ $=10.9 \mathrm{~Hz}, 1 \mathrm{H}), 4.91(\mathrm{~d}, J=11.6 \mathrm{~Hz}, 1 \mathrm{H}), 4.77(\mathrm{~d}, J=11.6 \mathrm{~Hz}, 1 \mathrm{H}), 4.74(\mathrm{~d}, J=10.9 \mathrm{~Hz}, 1 \mathrm{H})$, $4.44(\mathrm{~d}, J=10.3 \mathrm{~Hz}, 1 \mathrm{H}), 4.33(\mathrm{dd}, J=3.2,1.9 \mathrm{~Hz}, 1 \mathrm{H}), 4.12(\mathrm{dd}, J=8.9,3.3 \mathrm{~Hz}, 1 \mathrm{H}), 3.81$ $(\mathrm{dd}, J=12.8,3.4 \mathrm{~Hz}, 1 \mathrm{H}), 3.79-3.71(\mathrm{~m}, 2 \mathrm{H}), 3.68(\mathrm{dd}, J=12.8,2.1 \mathrm{~Hz}, 1 \mathrm{H}), 3.26(\mathrm{dd}, J=$ 10.7, 3.6 Hz, 1H), 3.24-3.15 (m, 2H), 2.13 (s, 3H), 2.03 (s, 3H), 2.02 (s, 3H), 1.78-1.69 (m, $2 \mathrm{H}), 1.29(\mathrm{~d}, J=5.7 \mathrm{~Hz}, 3 \mathrm{H}), 1.08(\mathrm{t}, J=7.3 \mathrm{~Hz}, 3 \mathrm{H}) ;{ }^{13} \mathrm{C} \mathrm{NMR}\left(151 \mathrm{MHz}, \mathrm{CDCl}_{3}\right) \delta 183.8$, 
$183.1,170.6,170.2,169.7,162.5,160.3,158.3,148.9,140.5,139.8,138.2,138.1,136.9,136.2$, $130.8(\mathrm{q}, J=32.6 \mathrm{~Hz}), 130.2(\mathrm{q}, J=32.4 \mathrm{~Hz}), 128.7,128.6,128.3,128.2,128.0,127.8,127.4$, 126.9, 125.9 (q, $J=3.6 \mathrm{~Hz}), 125.7(\mathrm{q}, J=3.6 \mathrm{~Hz}), 125.2,124.2(\mathrm{q}, J=272.0 \mathrm{~Hz}), 124.1(\mathrm{q}, J$ $=272.1 \mathrm{~Hz}), 119.5,112.1,107.8,103.6,96.8,94.6,80.1$, 78.1 , 75.7, 73.7, 73.3, 70.4, 69.9, 69.8, 68.3, 67.6, 61.2, 60.8, 37.4, 24.6, 20.9, 20.8, 20.8, 17.8, 14.5; HRMS (ESI-TOF) m/z [M + $\mathrm{Na}]^{+}$calcd for $\mathrm{C}_{65} \mathrm{H}_{61} \mathrm{~F}_{6} \mathrm{~N}_{3} \mathrm{O}_{16} \mathrm{Na}^{+} 1276.3848$, found 1276.3862 .
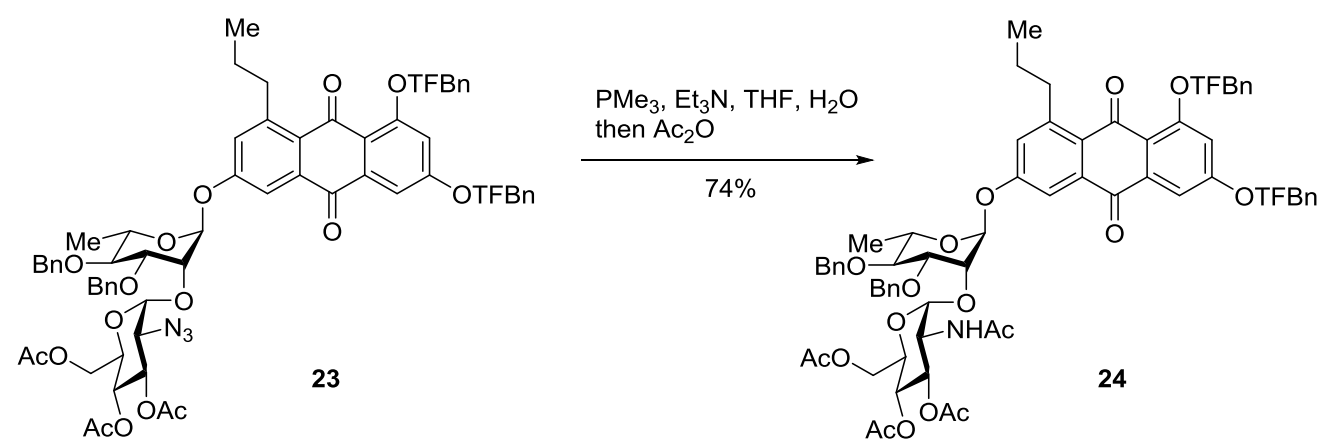

Compound 24. To the stirred solution of 23 (125 mg, $0.10 \mathrm{mmol})$ in THF (5 mL) was added $\mathrm{PMe}_{3}(0.50 \mathrm{~mL}, 1.0 \mathrm{M}$ in THF, $0.50 \mathrm{mmol})$ at room temperature under argon. The mixture was stirred at that temperature for $1.5 \mathrm{~h}$ before $\mathrm{Et}_{3} \mathrm{~N}(1.00 \mathrm{~mL})$ and water $(0.50 \mathrm{~mL})$ were added. The resulting mixture was stirred $10 \mathrm{~h}$ and then $\mathrm{Ac}_{2} \mathrm{O}(0.20 \mathrm{~mL}, 2.12 \mathrm{mmol})$ was added. The mixture was stirred for $3 \mathrm{~h}$ before it was quenched with methanol $(1.00 \mathrm{~mL})$. The resulting mixture was diluted with EtOAc, washed with aq. $\mathrm{HCl}(0.50 \mathrm{M})$ and brine, dried over anhydrous $\mathrm{Na}_{2} \mathrm{SO}_{4}$, filtered, and concentrated under vacuum. The residue was purified by silica gel chromatography (petroleum ether/acetone, 3:1) to afford $\mathbf{2 4}$ as a yellow foam (94 $\mathrm{mg}, 74 \%$ yield): $[\alpha]_{\mathrm{D}}^{25}+7.9\left(c 0.5, \mathrm{CHCl}_{3}\right) ;{ }^{1} \mathrm{H}$ NMR $\left(500 \mathrm{MHz}, \mathrm{CDCl}_{3}\right) \delta 7.74(\mathrm{~d}, J=7.8 \mathrm{~Hz}, 3 \mathrm{H}), 7.66$ $(\mathrm{d}, J=8.3 \mathrm{~Hz}, 4 \mathrm{H}), 7.55(\mathrm{~d}, J=8.1 \mathrm{~Hz}, 2 \mathrm{H}), 7.43-7.27(\mathrm{~m}, 11 \mathrm{H}), 7.12(\mathrm{~d}, J=2.6 \mathrm{~Hz}, 1 \mathrm{H})$, $6.85(\mathrm{~d}, J=2.3 \mathrm{~Hz}, 1 \mathrm{H}), 6.00(\mathrm{~d}, J=5.9 \mathrm{~Hz}, 1 \mathrm{H}), 5.50(\mathrm{~s}, 1 \mathrm{H}), 5.35-5.27(\mathrm{~m}, 3 \mathrm{H}), 5.23(\mathrm{~s}, 2 \mathrm{H})$, $5.18(\mathrm{t}, J=9.9 \mathrm{~Hz}, 1 \mathrm{H}), 5.06(\mathrm{~d}, J=3.1 \mathrm{~Hz}, 1 \mathrm{H}), 5.02(\mathrm{~d}, J=10.8 \mathrm{~Hz}, 1 \mathrm{H}), 4.81(\mathrm{~d}, J=2.1 \mathrm{~Hz}$, 2H), $4.75(\mathrm{~d}, J=10.8 \mathrm{~Hz}, 1 \mathrm{H}), 4.41-4.32(\mathrm{~m}, 2 \mathrm{H}), 4.31-4.27(\mathrm{~m}, 1 \mathrm{H}), 4.13(\mathrm{dd}, J=9.2,3.1$ $\mathrm{Hz}, 1 \mathrm{H}), 3.97(\mathrm{dd}, J=12.7,3.3 \mathrm{~Hz}, 1 \mathrm{H}), 3.82-3.74(\mathrm{~m}, 2 \mathrm{H}), 3.65(\mathrm{t}, J=9.3 \mathrm{~Hz}, 1 \mathrm{H}), 3.24-$ $3.12(\mathrm{~m}, 2 \mathrm{H}), 2.12(\mathrm{~s}, 3 \mathrm{H}), 2.08(\mathrm{~s}, 3 \mathrm{H}), 2.05(\mathrm{~s}, 3 \mathrm{H}), 2.03(\mathrm{~s}, 3 \mathrm{H}), 1.77-1.68(\mathrm{~m}, 2 \mathrm{H}), 1.33(\mathrm{~d}$, $J=6.1 \mathrm{~Hz}, 3 \mathrm{H}), 1.07(\mathrm{t}, J=7.3 \mathrm{~Hz}, 3 \mathrm{H}) ;{ }^{13} \mathrm{C} \mathrm{NMR}\left(126 \mathrm{MHz}, \mathrm{CDCl}_{3}\right) \delta 183.5,182.9,171.6$, 
$170.7,170.5,169.3,162.4,160.2,158.0,148.7,140.4,139.7,138.0,137.9,136.7,136.1,130.7$

$(\mathrm{q}, J=31.9 \mathrm{~Hz}), 130.1(\mathrm{q}, J=32.2 \mathrm{~Hz}), 128.7,128.6,128.4,128.2,128.03,128.00,127.7$, $127.3(\mathrm{~d}, J=273.5 \mathrm{~Hz}), 126.8,125.8(\mathrm{q}, J=3.7 \mathrm{~Hz}), 125.6(\mathrm{q}, J=3.6 \mathrm{~Hz}), 125.2,124.2(\mathrm{q}, J$ $=271.9 \mathrm{~Hz}), 124.0(\mathrm{q}, J=271.9 \mathrm{~Hz}), 119.3,111.3,107.7,103.4,96.5,94.9,80.0,77.9,75.6$, 73.7, 72.2, 71.4, 70.2, 69.7, 69.3, 68.3, 67.7, 61.3, 52.2, 37.4, 24.4, 23.3, 20.9, 20.8, 20.7, 18.4, 14.4; HRMS (ESI-TOF) $\mathrm{m} / \mathrm{z}[\mathrm{M}+\mathrm{Na}]^{+}$calcd for $\mathrm{C}_{67} \mathrm{H}_{65} \mathrm{~F}_{6} \mathrm{NO}_{17} \mathrm{Na}^{+}$1292.4049, found 1292.4038.
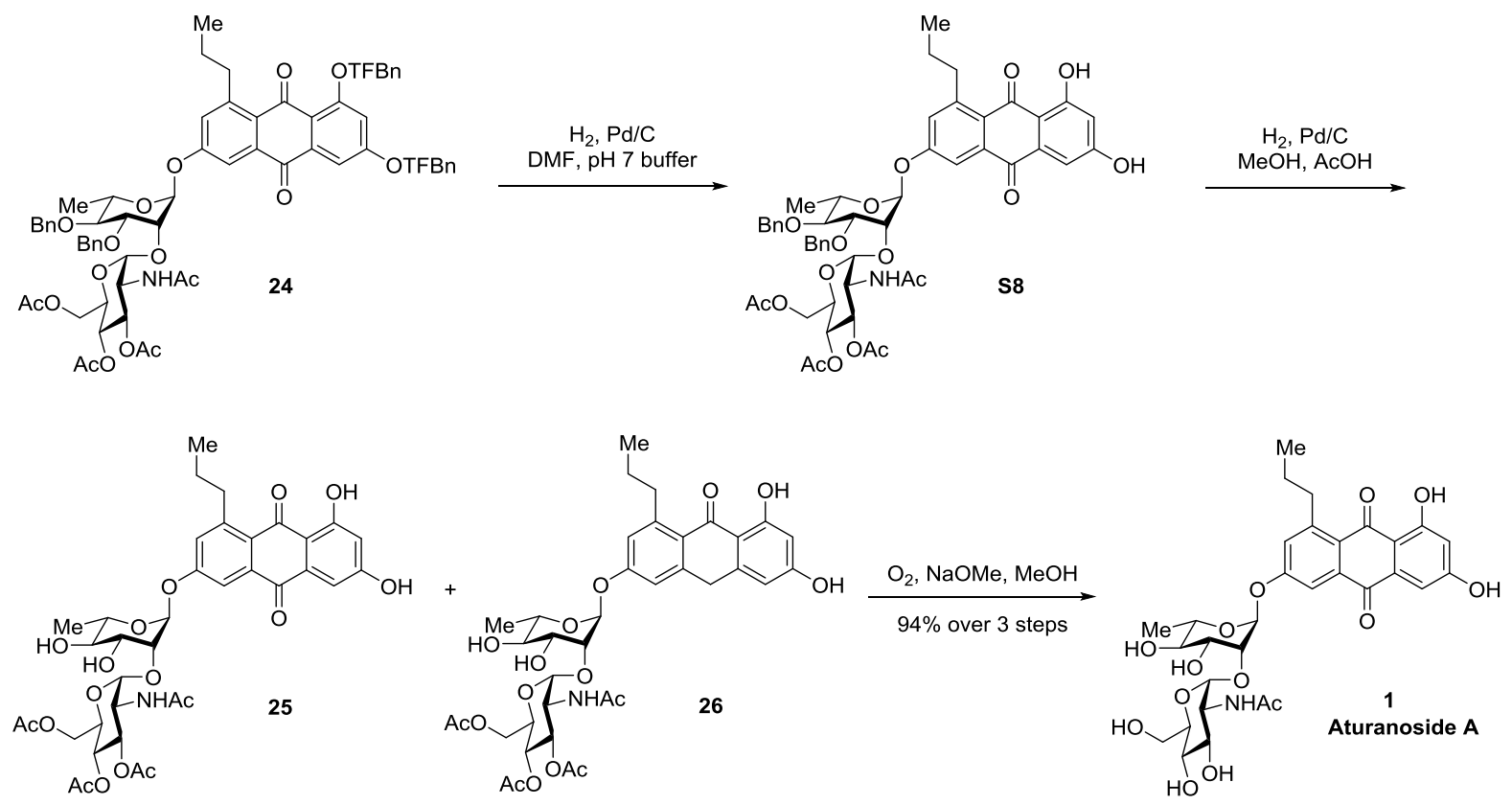

Aturanoside A (1). To a sirred solution of 24 (127 mg, $0.10 \mathrm{mmol})$ in DMF (20.0 mL) were added phosphate buffer $(5.00 \mathrm{~mL}, \mathrm{pH}=7)$ and $\mathrm{Pd} / \mathrm{C}(24.0 \mathrm{mg}, 10 \mathrm{wt} \%$ on carbon, $0.01 \mathrm{mmol})$. The mixture was stirred at $25{ }^{\circ} \mathrm{C}$ under a hydrogen atmosphere (balloon, $1 \mathrm{~atm}$ ) for $5 \mathrm{~h}$. The resulting mixture was concentrated under vacuum. The residue was diluted with EtOAc, washed with aq. $\mathrm{HCl}(0.50 \mathrm{M})$, saturated aq. $\mathrm{NaHCO}_{3}$, and brine, and was then dried over anhydrous $\mathrm{Na}_{2} \mathrm{SO}_{4}$, filtered, and concentrated under vacuum to afford crude $\mathbf{S 8}$ as a yellow solid. The crude $\mathbf{S 8}$ was used directly in the next step without further purification.

The crude S8 was dissolved in methanol $(20.0 \mathrm{~mL})$, then acetic acid $(2.00 \mathrm{~mL})$ and $\mathrm{Pd} / \mathrm{C}$ (10 wt $\%$ on carbon, $24.0 \mathrm{mg}, 0.01 \mathrm{mmol}$ ) were added. The mixture was stirred at $25{ }^{\circ} \mathrm{C}$ under a hydrogen atmosphere (balloon, $1 \mathrm{~atm}$ ) for $40 \mathrm{~h}$. The resulting mixture was filtered through a 
pad of celite and washed with EtOAc three times. The filtrate was concentrated under vacuum to afford a crude mixture of 25 and $26(\mathbf{2 5 / 2 6}=1: 1)$ as an amorphous yellow solid. The crude mixture of $\mathbf{2 5}$ and $\mathbf{2 6}$ was used directly in the next step without further purification.

The crude mixture of $\mathbf{2 5}$ and $\mathbf{2 6}$ was dissolved in methanol (20.0 mL), then sodium methoxide (54 mg, $1.00 \mathrm{mmol}, 0.05 \mathrm{M}$ in methanol) was added. The mixture was stirred at $25{ }^{\circ} \mathrm{C}$ under an oxygen atmosphere (balloon, $1 \mathrm{~atm}$ ) for $3 \mathrm{~h}$. The resulting mixture was neutralized with ion-exchange resin (hydrogen form), filtered, and concentrated under vacuum. The residue was purified by silica gel chromatography $\left(\mathrm{CH}_{2} \mathrm{Cl}_{2} /\right.$ methanol, 4:1) to afford Aturanoside A (1) as a yellow foam (61 mg, 94\% yield over 3 steps).

Compound S8: $[\alpha]_{\mathrm{D}}^{25}-85.2\left(c\right.$ 0.2, $\left.\mathrm{CHCl}_{3}\right) ;{ }^{1} \mathrm{H} \mathrm{NMR}\left(500 \mathrm{MHz}, \mathrm{CDCl}_{3}\right) \delta{ }^{1} \mathrm{H} \mathrm{NMR}(500$ $\left.\mathrm{MHz}, \mathrm{CDCl}_{3}\right) \delta 12.87(\mathrm{~s}, 1 \mathrm{H}), 10.15(\mathrm{~s}, 1 \mathrm{H}), 7.35-7.27(\mathrm{~m}, 8 \mathrm{H}), 7.23(\mathrm{dd}, J=9.4,2.7 \mathrm{~Hz}, 2 \mathrm{H})$, $6.85(\mathrm{~d}, J=2.4 \mathrm{~Hz}, 1 \mathrm{H}), 6.73(\mathrm{~d}, J=2.7 \mathrm{~Hz}, 1 \mathrm{H}), 6.42(\mathrm{~d}, J=2.4 \mathrm{~Hz}, 1 \mathrm{H}), 6.32(\mathrm{~d}, J=9.0 \mathrm{~Hz}$, 1H), $5.54(\mathrm{~d}, J=3.7 \mathrm{~Hz}, 1 \mathrm{H}), 5.43(\mathrm{~d}, J=2.0 \mathrm{~Hz}, 1 \mathrm{H}), 5.31(\mathrm{dd}, J=10.7,9.7 \mathrm{~Hz}, 1 \mathrm{H}), 5.18(\mathrm{t}$, $J=9.9 \mathrm{~Hz}, 1 \mathrm{H}), 4.96(\mathrm{~d}, J=10.8 \mathrm{~Hz}, 1 \mathrm{H}), 4.78(\mathrm{~s}, 2 \mathrm{H}), 4.68(\mathrm{~d}, J=10.9 \mathrm{~Hz}, 1 \mathrm{H}), 4.49(\mathrm{dd}, J$ $=3.3,1.9 \mathrm{~Hz}, 1 \mathrm{H}), 4.45(\mathrm{ddd}, J=10.7,9.1,3.7 \mathrm{~Hz}, 1 \mathrm{H}), 4.34(\mathrm{dt}, J=10.3,2.7 \mathrm{~Hz}, 1 \mathrm{H}), 4.13$ $(\mathrm{dd}, J=9.1,3.2 \mathrm{~Hz}, 1 \mathrm{H}), 3.97(\mathrm{dd}, J=12.8,3.1 \mathrm{~Hz}, 1 \mathrm{H}), 3.78(\mathrm{dd}, J=12.8,2.2 \mathrm{~Hz}, 1 \mathrm{H}), 3.70$ $3.63(\mathrm{~m}, 1 \mathrm{H}), 3.59(\mathrm{t}, J=9.3 \mathrm{~Hz}, 1 \mathrm{H}), 2.81(\mathrm{ddd}, J=14.7,9.1,5.9 \mathrm{~Hz}, 1 \mathrm{H}), 2.53-2.42(\mathrm{~m}, 1 \mathrm{H})$, $2.14(\mathrm{~s}, 3 \mathrm{H}), 2.06(\mathrm{~s}, 3 \mathrm{H}), 2.00(\mathrm{~s}, 3 \mathrm{H}), 1.99(\mathrm{~s}, 3 \mathrm{H}), 1.29-1.20(\mathrm{~m}, 5 \mathrm{H}), 0.79(\mathrm{t}, J=7.3 \mathrm{~Hz}$, $3 \mathrm{H}) ;{ }^{13} \mathrm{C} \mathrm{NMR}\left(126 \mathrm{MHz}, \mathrm{CDCl}_{3}\right) \delta 187.3,181.9,173.4,171.9,170.8,169.4,165.4,164.3$, $158.7,149.7,138.2,138.0,136.3,134.0,128.7,128.6,128.1,128.00,127.98,127.4,124.6$, $110.8,109.1,107.2,96.1,95.0,80.2,78.0,75.6,73.8,72.2,71.1,69.2,68.4,67.5,61.28,61.27$, 53.0, 37.7, 23.2, 22.8, 21.0, 20.9, 20.8, 18.5, 14.2; HRMS (ESI-TOF) m/z [M + Na $]^{+}$calcd for $\mathrm{C}_{51} \mathrm{H}_{55} \mathrm{NO}_{17} \mathrm{Na}^{+}$976.3362, found 976.3357.

Compound 25: $[\alpha]_{\mathrm{D}}^{25}-87.4\left(c 0.1, \mathrm{CHCl}_{3}\right) ;{ }^{1} \mathrm{H}$ NMR (600 MHz, acetone- $\left.d_{6}\right) \delta 13.19$ (s, $1 \mathrm{H}), 10.21(\mathrm{~s}, 1 \mathrm{H}), 7.74(\mathrm{~d}, J=2.8 \mathrm{~Hz}, 1 \mathrm{H}), 7.25(\mathrm{~d}, J=2.8 \mathrm{~Hz}, 1 \mathrm{H}), 7.19(\mathrm{~d}, J=2.5 \mathrm{~Hz}, 1 \mathrm{H})$, $7.02(\mathrm{~d}, J=8.8 \mathrm{~Hz}, 1 \mathrm{H}), 6.65(\mathrm{~d}, J=2.4 \mathrm{~Hz}, 1 \mathrm{H}), 5.86(\mathrm{~d}, J=2.0 \mathrm{~Hz}, 1 \mathrm{H}), 5.41(\mathrm{~d}, J=3.8 \mathrm{~Hz}$, 1H), 5.32 (dd, $J=11.0,9.4 \mathrm{~Hz}, 1 \mathrm{H}), 5.07$ (dd, $J=10.4,9.4 \mathrm{~Hz}, 1 \mathrm{H}), 4.64$ (ddd, $J=10.4,4.4$, $2.5 \mathrm{~Hz}, 1 \mathrm{H}), 4.45(\mathrm{~d}, J=5.9 \mathrm{~Hz}, 1 \mathrm{H}), 4.33-4.28(\mathrm{~m}, 2 \mathrm{H}), 4.25-4.20(\mathrm{~m}, 2 \mathrm{H}), 4.15(\mathrm{dd}, J=12.3$, 
$2.4 \mathrm{~Hz}, 1 \mathrm{H}), 4.09(\mathrm{ddd}, J=9.4,5.8,3.4 \mathrm{~Hz}, 1 \mathrm{H}), 3.75(\mathrm{td}, J=9.3,5.1 \mathrm{~Hz}, 1 \mathrm{H}), 3.66(\mathrm{dq}, J=$ 9.2, $6.1 \mathrm{~Hz}, 1 \mathrm{H}), 3.22(\mathrm{ddd}, J=12.7,9.3,6.0 \mathrm{~Hz}, 1 \mathrm{H}), 3.01$ (ddd, $J=12.8,9.2,6.2 \mathrm{~Hz}, 1 \mathrm{H})$, $2.04(\mathrm{~s}, 3 \mathrm{H}), 2.02(\mathrm{~s}, 3 \mathrm{H}), 2.01(\mathrm{~s}, 3 \mathrm{H}), 1.96(\mathrm{~s}, 3 \mathrm{H}), 1.66-1.57(\mathrm{~m}, 2 \mathrm{H}), 1.27(\mathrm{~d}, J=6.1 \mathrm{~Hz}$, 3H), $0.99(\mathrm{t}, J=7.3 \mathrm{~Hz}, 3 \mathrm{H}) ;{ }^{13} \mathrm{C}$ NMR $\left(151 \mathrm{MHz}\right.$, acetone- $\left.d_{6}\right) \delta$ 189.3, 182.9, 171.2, 170.9, $170.8,170.0,166.5,165.2,160.7,150.5,138.0,135.6,125.6,125.4,114.0,111.8,109.3,107.9$, $96.53,96.48,76.8,73.3,71.7,71.3,71.2,69.7,68.8,62.6,52.9,38.5,24.6,22.9,20.73,20.69$, 18.1, 14.5; HRMS (ESI-TOF) $\mathrm{m} / \mathrm{z}[\mathrm{M}+\mathrm{Na}]^{+}$calcd for $\mathrm{C}_{37} \mathrm{H}_{43} \mathrm{NO}_{17} \mathrm{Na}^{+}$796.2423, found 796.2425 .

Compound 26: $[\alpha]_{\mathrm{D}}^{25}+23.7\left(c 0.2, \mathrm{CHCl}_{3}\right) ;{ }^{1} \mathrm{H}$ NMR $\left(600 \mathrm{MHz}\right.$, acetone- $\left.d_{6}\right) \delta 13.57(\mathrm{~s}$, 1H), $7.07(\mathrm{~d}, J=2.4 \mathrm{~Hz}, 1 \mathrm{H}), 6.98(\mathrm{~d}, J=2.6 \mathrm{~Hz}, 1 \mathrm{H}), 6.75(\mathrm{~d}, J=9.1 \mathrm{~Hz}, 1 \mathrm{H}), 6.43(\mathrm{dt}, J=$ 2.3, 1.1 Hz, 1H), $6.27(\mathrm{~d}, J=2.3 \mathrm{~Hz}, 1 \mathrm{H}), 5.83(\mathrm{~d}, J=1.9 \mathrm{~Hz}, 1 \mathrm{H}), 5.31-5.26(\mathrm{~m}, 2 \mathrm{H}), 5.05$ $(\mathrm{dd}, J=10.4,9.5 \mathrm{~Hz}, 1 \mathrm{H}), 4.59$ (ddd, $J=10.4,4.5,2.5 \mathrm{~Hz}, 1 \mathrm{H}), 4.32(\mathrm{~s}, 2 \mathrm{H}), 4.25$ (ddd, $J=$ $10.9,9.1,3.8 \mathrm{~Hz}, 1 \mathrm{H}), 4.22-4.18(\mathrm{~m}, 2 \mathrm{H}), 4.12(\mathrm{dd}, J=12.3,2.4 \mathrm{~Hz}, 1 \mathrm{H}), 4.04(\mathrm{dd}, J=8.8$, $3.3 \mathrm{~Hz}, 1 \mathrm{H}), 3.73-3.64(\mathrm{~m}, 2 \mathrm{H}), 3.20(\mathrm{ddt}, J=34.1,12.3,7.6 \mathrm{~Hz}, 2 \mathrm{H}), 2.01(\mathrm{~s}, 3 \mathrm{H}), 2.00(\mathrm{~s}$, $3 \mathrm{H}), 1.95(\mathrm{~s}, 3 \mathrm{H}), 1.95(\mathrm{~s}, 3 \mathrm{H}), 1.70-1.62(\mathrm{~m}, 2 \mathrm{H}), 1.28(\mathrm{~d}, J=5.8 \mathrm{~Hz}, 3 \mathrm{H}), 1.01(\mathrm{t}, J=7.3 \mathrm{~Hz}$, $3 \mathrm{H}) ;{ }^{13} \mathrm{C}$ NMR $\left(151 \mathrm{MHz}\right.$, acetone- $\left.d_{6}\right) \delta 190.5,170.9,170.8,170.3,170.0,166.5,164.3,159.8$, $149.7,146.1,144.5,124.2,119.6,114.3,112.1,106.6,101.8,96.6,96.2,76.8,73.5,71.8,71.2$, 71.1, 69.6, 68.8, 62.6, 52.7, 39.2, 34.6, 25.4, 23.0, 20.71, 20.68, 20.67, 18.2, 14.6; HRMS (ESITOF) $\mathrm{m} / \mathrm{z}[\mathrm{M}+\mathrm{H}]^{+}$calcd for $\mathrm{C}_{37} \mathrm{H}_{46} \mathrm{NO}_{16}{ }^{+} 760.2811$, found 760.2813 .

Compound 1: $[\alpha]_{\mathrm{D}}^{25}+20.8(c 0.1, \mathrm{MeOH}) ;{ }^{1} \mathrm{H} \mathrm{NMR}\left(600 \mathrm{MHz}, \mathrm{CD}_{3} \mathrm{OD}\right) \delta 7.66(\mathrm{~d}, J=2.7$ $\mathrm{Hz}, 1 \mathrm{H}), 7.11(\mathrm{~d}, J=2.8 \mathrm{~Hz}, 1 \mathrm{H}), 7.03(\mathrm{~d}, J=2.4 \mathrm{~Hz}, 1 \mathrm{H}), 6.50(\mathrm{~d}, J=2.4 \mathrm{~Hz}, 1 \mathrm{H}), 5.65(\mathrm{~d}, J$ $=1.9 \mathrm{~Hz}, 1 \mathrm{H}), 5.26(\mathrm{~d}, J=3.7 \mathrm{~Hz}, 1 \mathrm{H}), 4.17(\mathrm{dd}, J=3.5,1.9 \mathrm{~Hz}, 1 \mathrm{H}), 4.04(\mathrm{ddd}, J=10.0,5.2$, $2.4 \mathrm{~Hz}, 1 \mathrm{H}), 4.01(\mathrm{dd}, J=9.1,3.5 \mathrm{~Hz}, 1 \mathrm{H}), 3.93(\mathrm{dd}, J=10.8,3.7 \mathrm{~Hz}, 1 \mathrm{H}), 3.88(\mathrm{dd}, J=12.0$, $2.4 \mathrm{~Hz}, 1 \mathrm{H}), 3.79(\mathrm{dd}, J=10.8,8.9 \mathrm{~Hz}, 1 \mathrm{H}), 3.76(\mathrm{dd}, J=12.0,5.1 \mathrm{~Hz}, 1 \mathrm{H}), 3.60(\mathrm{dq}, J=9.3$, $6.0 \mathrm{~Hz}, 1 \mathrm{H}), 3.56(\mathrm{t}, J=9.2 \mathrm{~Hz}, 1 \mathrm{H}), 3.45(\mathrm{dd}, J=10.0,8.9 \mathrm{~Hz}, 1 \mathrm{H}), 3.14(\mathrm{ddd}, J=12.8,8.9$, $6.3 \mathrm{~Hz}, 1 \mathrm{H}), 3.01(\mathrm{ddd}, J=12.8,8.9,6.6 \mathrm{~Hz}, 1 \mathrm{H}), 2.12(\mathrm{~s}, 3 \mathrm{H}), 1.65-1.55(\mathrm{~m}, 2 \mathrm{H}), 1.29(\mathrm{~d}, J$ $=6.0 \mathrm{~Hz}, 3 \mathrm{H}), 1.01(\mathrm{t}, J=7.3 \mathrm{~Hz}, 3 \mathrm{H}) ;{ }^{13} \mathrm{C} \mathrm{NMR}\left(151 \mathrm{MHz}, \mathrm{CD}_{3} \mathrm{OD}\right) \delta 189.3,183.5,174.0$, $166.5,165.8,160.7,150.9,138.4,135.7,126.1,125.9,113.8,111.7,109.4,108.5,97.1,96.8$, 
77.0, 74.0, 73.8, 72.3, 72.1, 71.5, 71.4, 62.5, 55.8, 39.1, 25.1, 22.8, 18.3, 14.7; HRMS (ESITOF) $\mathrm{m} / \mathrm{z}[\mathrm{M}+\mathrm{Na}]^{+}$calcd for $\mathrm{C}_{31} \mathrm{H}_{37} \mathrm{NO}_{14} \mathrm{Na}^{+}$670.2106, found 670.2108.
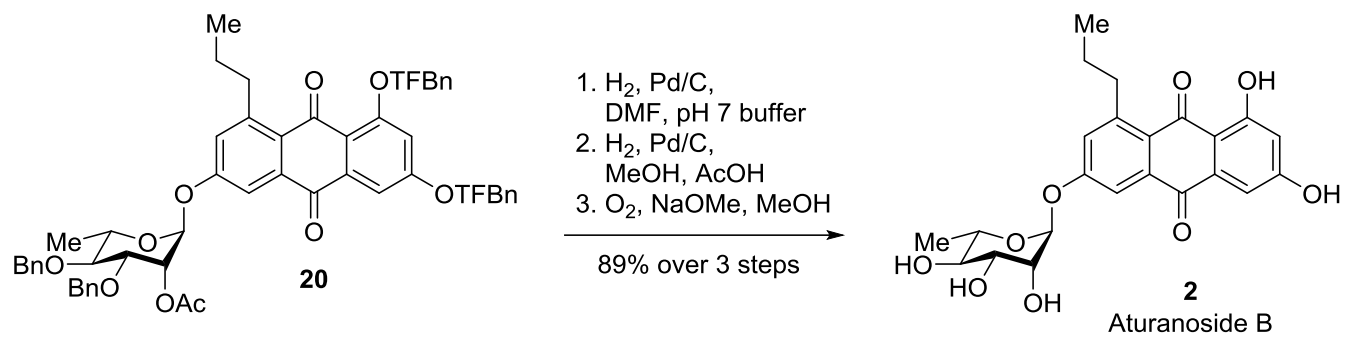

Aturanoside B (2). To a sirred solution of compound 20 (94 mg, $0.10 \mathrm{mmol}$ ) in DMF (20.0 $\mathrm{mL})$ were added phosphate buffer $(\mathrm{pH}=7,4.00 \mathrm{~mL})$ and $\mathrm{Pd} / \mathrm{C}(10 \mathrm{wt} \%$ on carbon, $24.0 \mathrm{mg}$, $0.01 \mathrm{mmol}$ ). The mixture was stirred at $25{ }^{\circ} \mathrm{C}$ under a hydrogen atmosphere (balloon, $1 \mathrm{~atm}$ ) for $4 \mathrm{~h}$. The resulting mixture was concentrated under vacuum. The residue was diluted with EtOAc, washed with aq. $\mathrm{HCl}(0.50 \mathrm{M})$, saturated aq. $\mathrm{NaHCO}_{3}$, and brine, and was then dried over anhydrous $\mathrm{Na}_{2} \mathrm{SO}_{4}$, filtered, and concentrated under vacuum.

The residue was dissolved in methanol $(20.0 \mathrm{~mL})$, then acetic acid $(2.00 \mathrm{~mL})$ and $\mathrm{Pd} / \mathrm{C}$ (10 wt $\%$ on carbon, $24.0 \mathrm{mg}, 0.01 \mathrm{mmol}$ ) were added. The mixture was stirred at $25^{\circ} \mathrm{C}$ under a hydrogen atmosphere (balloon, $1 \mathrm{~atm}$ ) for $5 \mathrm{~h}$. The resulting mixture was filtered through a pad of celite and washed with EtOAc three times. The filtrate was concentrated under vacuum.

The resultant residue was dissolved in methanol $(20.0 \mathrm{~mL})$, then sodium methoxide (54 $\mathrm{mg}, 1.00 \mathrm{mmol}, 0.05 \mathrm{M}$ in methanol) was added. The mixture was stirred at $25{ }^{\circ} \mathrm{C}$ under an oxygen atmosphere (balloon, $1 \mathrm{~atm}$ ) for $3 \mathrm{~h}$. The resulting mixture was neutralized with ionexchange resin (hydrogen form), filtered, and concentrated under vacuum. The residue was purified by silica gel chromatography $\left(\mathrm{CH}_{2} \mathrm{Cl}_{2} /\right.$ methanol, 5:1) to afford aturanoside $\mathrm{B}(2)$ as a yellow foam (38 mg, 89\% yield over 3 steps): $[\alpha]_{\mathrm{D}}^{25}-119.0(c$ 0.2, $\mathrm{MeOH}) ;{ }^{1} \mathrm{H} \mathrm{NMR}(500 \mathrm{MHz}$, $\left.\mathrm{CD}_{3} \mathrm{OD}\right) \delta 7.76(\mathrm{~d}, J=2.7 \mathrm{~Hz}, 1 \mathrm{H}), 7.22(\mathrm{~d}, J=2.7 \mathrm{~Hz}, 1 \mathrm{H}), 7.09(\mathrm{~d}, J=2.5 \mathrm{~Hz}, 1 \mathrm{H}), 6.54(\mathrm{~d}$, $J=2.5 \mathrm{~Hz}, 1 \mathrm{H}), 5.64(\mathrm{~d}, J=1.7 \mathrm{~Hz}, 1 \mathrm{H}), 4.07(\mathrm{dd}, J=3.5,1.8 \mathrm{~Hz}, 1 \mathrm{H}), 3.87(\mathrm{dd}, J=9.4,3.5$ $\mathrm{Hz}, 1 \mathrm{H}), 3.58(\mathrm{dq}, J=9.2,6.1 \mathrm{~Hz}, 1 \mathrm{H}), 3.50(\mathrm{t}, J=9.4 \mathrm{~Hz}, 1 \mathrm{H}), 3.25-3.18(\mathrm{~m}, 1 \mathrm{H}), 3.18-3.11$ (m, 1H), 1.71-1.62 (m, 2H), $1.25(\mathrm{~d}, J=6.1 \mathrm{~Hz}, 3 \mathrm{H}), 1.04(\mathrm{t}, J=7.3 \mathrm{~Hz}, 3 \mathrm{H}) ;{ }^{13} \mathrm{C} \mathrm{NMR}(126$ $\left.\mathrm{MHz}, \mathrm{CD}_{3} \mathrm{OD}\right) \delta 189.5,183.8,166.5,165.8,160.9,151.1,138.6,135.9,126.11,126.06,113.8$, 
111.8, 109.4, 108.5, 99.6, 73.6, 72.1, 71.7, 71.2, 39.1, 25.3, 18.1, 14.7; HRMS (ESI-TOF) m/z $[\mathrm{M}-\mathrm{H}]^{-}$calcd for $\mathrm{C}_{23} \mathrm{H}_{23} \mathrm{O}_{9}{ }^{-} 443.1348$, found 443.1342 . 
II Comparison of the Spectroscopic Data of the Authentic and Synthetic Aturanosides

Table S1. Comparison of the ${ }^{1} \mathrm{H}$ NMR spectroscopic data $\left(\mathrm{CD}_{3} \mathrm{OD}\right)$ of the authentic and synthetic Aturanoside A (1).

\begin{tabular}{|c|c|c|}
\hline $\begin{array}{l}\text { Authentic }^{a} \\
\delta_{\mathrm{H}}(\mathrm{ppm})\end{array}$ & $\begin{array}{l}\text { Synthetic }^{b, c} \\
\delta_{\mathrm{H}}(\mathrm{ppm})\end{array}$ & $\begin{array}{c}\operatorname{Err}(\text { Synthetic }- \text { Authentic }) \\
\Delta \delta_{\mathrm{H}}(\mathrm{ppm})\end{array}$ \\
\hline 7.76 & 7.66 & -0.10 \\
\hline 7.19 & 7.11 & -0.09 \\
\hline 7.09 & 7.03 & -0.06 \\
\hline 6.53 & 6.50 & -0.03 \\
\hline 5.68 & 5.65 & -0.03 \\
\hline 5.22 & 5.26 & 0.04 \\
\hline 4.15 & 4.17 & 0.02 \\
\hline 4.03 & 4.04 & 0.01 \\
\hline 4.00 & 4.01 & 0.01 \\
\hline 3.90 & 3.93 & 0.03 \\
\hline 3.86 & 3.88 & 0.02 \\
\hline 3.78 & 3.79 & 0.01 \\
\hline 3.74 & 3.76 & 0.02 \\
\hline 3.60 & 3.60 & 0 \\
\hline 3.55 & 3.56 & 0.01 \\
\hline 3.43 & 3.45 & 0.02 \\
\hline 3.23 & 3.14 & -0.09 \\
\hline 3.13 & 3.01 & -0.12 \\
\hline 2.10 & 2.12 & 0.02 \\
\hline 1.65 & 1.60 & -0.05 \\
\hline 1.29 & 1.29 & 0 \\
\hline 1.03 & 1.01 & -0.02 \\
\hline
\end{tabular}

${ }^{a}$ Data recorded at $700 \mathrm{MHz} .{ }^{b}$ Data recorded at $600 \mathrm{MHz} .{ }^{c}$ Multiplets are expressed as the middle value of its chemical shift range. 
Table S2. Comparison of the ${ }^{13} \mathrm{C}$ NMR spectroscopic data $\left(\mathrm{CD}_{3} \mathrm{OD}\right)$ of authentic and synthetic Aturanoside A (1).

\begin{tabular}{|c|c|c|}
\hline $\begin{array}{l}\text { Authentic }^{a} \\
\delta_{C}(\mathrm{ppm})\end{array}$ & $\begin{array}{l}\text { Synthetic }^{b} \\
\delta_{C}(\mathrm{ppm})\end{array}$ & $\begin{array}{c}\operatorname{Err}(\text { Synthetic }- \text { Authentic }) \\
\Delta \delta_{\mathrm{C}}(\mathrm{ppm})\end{array}$ \\
\hline 189.5 & 189.3 & -0.2 \\
\hline 183.9 & 183.5 & -0.4 \\
\hline 174.0 & 174.0 & 0 \\
\hline 166.7 & 166.5 & -0.2 \\
\hline 166.5 & 165.8 & -0.7 \\
\hline 160.9 & 160.7 & -0.2 \\
\hline 151.1 & 150.9 & -0.2 \\
\hline $138.7^{c}$ & 138.4 & -0.3 \\
\hline 135.9 & 135.7 & -0.2 \\
\hline 126.4 & 126.1 & -0.3 \\
\hline 126.2 & 125.9 & -0.3 \\
\hline 113.9 & 113.8 & -0.1 \\
\hline 111.8 & 111.7 & -0.1 \\
\hline 109.6 & 109.4 & -0.2 \\
\hline 108.9 & 108.5 & -0.4 \\
\hline 97.3 & 97.1 & -0.2 \\
\hline 96.9 & 96.8 & -0.1 \\
\hline 77.1 & 77.0 & -0.1 \\
\hline 74.1 & 74.0 & -0.1 \\
\hline 73.9 & 73.8 & -0.1 \\
\hline 72.5 & 72.3 & -0.2 \\
\hline 72.2 & 72.1 & -0.1 \\
\hline 71.6 & 71.5 & -0.1 \\
\hline
\end{tabular}




\begin{tabular}{|l|l|l|}
\hline 71.5 & 71.4 & -0.1 \\
\hline 62.6 & 62.5 & -0.1 \\
\hline 55.9 & 55.8 & -0.1 \\
\hline 39.2 & 39.1 & -0.1 \\
\hline 25.3 & 25.1 & -0.2 \\
\hline 22.9 & 22.8 & -0.1 \\
\hline 18.4 & 18.3 & -0.1 \\
\hline 14.7 & 14.7 & 0 \\
\hline
\end{tabular}

${ }^{a}$ Data recorded at $175 \mathrm{MHz} .{ }^{b}$ Data recorded at $151 \mathrm{MHz} .{ }^{c}$ This data was not listed in the literature $;{ }^{\mathrm{S} 7}$ it was measured based on the spectrum provided in the supporting information of the literature. 
Table S3. Comparison of the ${ }^{1} \mathrm{H}$ NMR spectroscopic data $\left(\mathrm{CD}_{3} \mathrm{OD}\right)$ of authentic and synthetic Aturanoside B (2).

\begin{tabular}{|c|c|c|}
\hline $\begin{array}{l}\text { Authentic }^{a} \\
\delta_{\mathrm{H}}(\mathrm{ppm})\end{array}$ & $\begin{array}{l}\text { Synthetic }^{b, c} \\
\delta_{\mathrm{H}}(\mathrm{ppm})\end{array}$ & $\begin{array}{c}\operatorname{Err}(\text { Synthetic }- \text { Authentic }) \\
\Delta \delta_{\mathrm{H}}(\mathrm{ppm})\end{array}$ \\
\hline 7.80 & 7.76 & -0.04 \\
\hline 7.24 & 7.22 & -0.02 \\
\hline 7.13 & 7.09 & -0.04 \\
\hline 6.55 & 6.54 & -0.01 \\
\hline 5.64 & 5.64 & 0 \\
\hline 4.06 & 4.07 & 0.01 \\
\hline 3.86 & 3.87 & 0.01 \\
\hline 3.57 & 3.58 & 0.01 \\
\hline 3.50 & 3.50 & 0 \\
\hline 3.23 & 3.22 & -0.01 \\
\hline 3.17 & 3.14 & -0.03 \\
\hline 1.67 & 1.66 & -0.01 \\
\hline 1.24 & 1.25 & 0.01 \\
\hline 1.04 & 1.04 & 0 \\
\hline
\end{tabular}

${ }^{a}$ Data recorded at $800 \mathrm{MHz} .{ }^{b}$ Data recorded at $500 \mathrm{MHz} .{ }^{c}$ Multiplets are expressed as the middle value of its chemical shift range. 
Table S4. Comparison of the ${ }^{13} \mathrm{C}$ NMR spectroscopic data $\left(\mathrm{CD}_{3} \mathrm{OD}\right)$ of authentic and synthetic Aturanoside B (2).

\begin{tabular}{|c|c|c|}
\hline $\begin{array}{l}\text { Authentic } \\
\delta_{\mathrm{C}}(\mathrm{ppm})\end{array}$ & $\begin{array}{l}\text { Synthetic } \\
\delta_{C}(\mathrm{ppm})\end{array}$ & $\begin{array}{c}\operatorname{Err}(\text { Synthetic }- \text { Authentic }) \\
\Delta \delta_{\mathrm{C}}(\mathrm{ppm})\end{array}$ \\
\hline 189.7 & 189.5 & -0.2 \\
\hline 184.1 & 183.8 & -0.2 \\
\hline 166.7 & 166.5 & -0.2 \\
\hline 166.1 & 165.8 & -0.3 \\
\hline 161.0 & 160.9 & -0.1 \\
\hline 151.2 & 151.1 & -0.1 \\
\hline 138.8 & 138.6 & -0.2 \\
\hline 136.0 & 135.9 & -0.1 \\
\hline 126.3 & 126.11 & -0.2 \\
\hline 126.2 & 126.06 & -0.1 \\
\hline 114.0 & 113.8 & -0.2 \\
\hline 111.9 & 111.8 & -0.1 \\
\hline 109.5 & 109.4 & -0.1 \\
\hline 108.7 & 108.5 & -0.2 \\
\hline 99.8 & 99.6 & -0.2 \\
\hline 73.7 & 73.6 & -0.1 \\
\hline 72.2 & 72.1 & -0.1 \\
\hline 71.8 & 71.7 & -0.1 \\
\hline 71.3 & 71.2 & -0.1 \\
\hline 39.2 & 39.1 & -0.1 \\
\hline 25.4 & 25.3 & -0.1 \\
\hline 18.2 & 18.1 & -0.1 \\
\hline 14.7 & 14.7 & 0 \\
\hline
\end{tabular}

${ }^{a}$ Data recorded at $200 \mathrm{MHz} .{ }^{b}$ Data recorded at $126 \mathrm{MHz}$. 


\section{References}

(1) Cai, L.; Zeng, J.; Li, T.; Xiao, Y.; Ma, X.; Xiao, X.; Zhang, Q.; Meng, L.; Wan, Q. Dehydrative glycosylation enabled by a comproportionation reaction of 2-aryl-1,3dithiane 1-oxide. Chin. J. Chem. 2020, 38, 43-49.

(2) Yu, B.; Cao, X. 2-(1-Hexyn-1-yl)benzoic Acid. In E-EROS encyclopedia of reagents for organic synthesis; Wiley, 2014. DOI: 10.1002/047084289X.rn01722.

(3) Barroso, S.; Geerdink, D.; ter Horst, B.; Casas-Arce, E.; Minnaard, A. J. Total synthesis of the phenolic glycolipid Mycoside B and the glycosylated phydroxybenzoic acid methyl ester HBAD-I, virulence markers of mycobacterium tuberculosis. Eur. J. Org. Chem. 2013, 4642-4654.

(4) Ma, Y.; Cao, X.; Yu, B. Synthesis of oligosaccharide fragments of the rhamnogalacturonan of Nerium indicum. Carbohydr. Res. 2013, 377, 63-74.

(5) Palomino, J. C. C.; Rensoli, M. H.; Bencomo, V. V. Synthesis of the trisaccharide $\alpha$ L-Rha-(1-2)- $\alpha$-L-Rha-(1-2)- $\alpha$-L-Rha with a dioxolane-type spacer-arm. J. Carbohydr. Chem. 1996, 15, 137-146.

(6) Yang, Y.; Li, Y.; Yu, B. Total synthesis and structural revision of TMG-chitotriomycin, a specific inhibitor of insect and fungal $\beta-N$-acetylglucosaminidases. J. Am. Chem. Soc. 2009, 131, 12076-12077.

(7) Jang, J.-P.; Hwang, G. J.; Jang, M.; Takahashi, S.; Ko, S.-K.; Osada, H.; Jang, J.-H.; Ahn, J. S. Aturanosides A and B, glycosylated anthraquinones with antiangiogenic activity from a soil-derived Streptomyces species. J. Nat. Prod. 2018, 81, 2004-2009. 
IV ${ }^{1} \mathrm{H}$ and ${ }^{13} \mathrm{C}$ NMR Spectra of Compounds

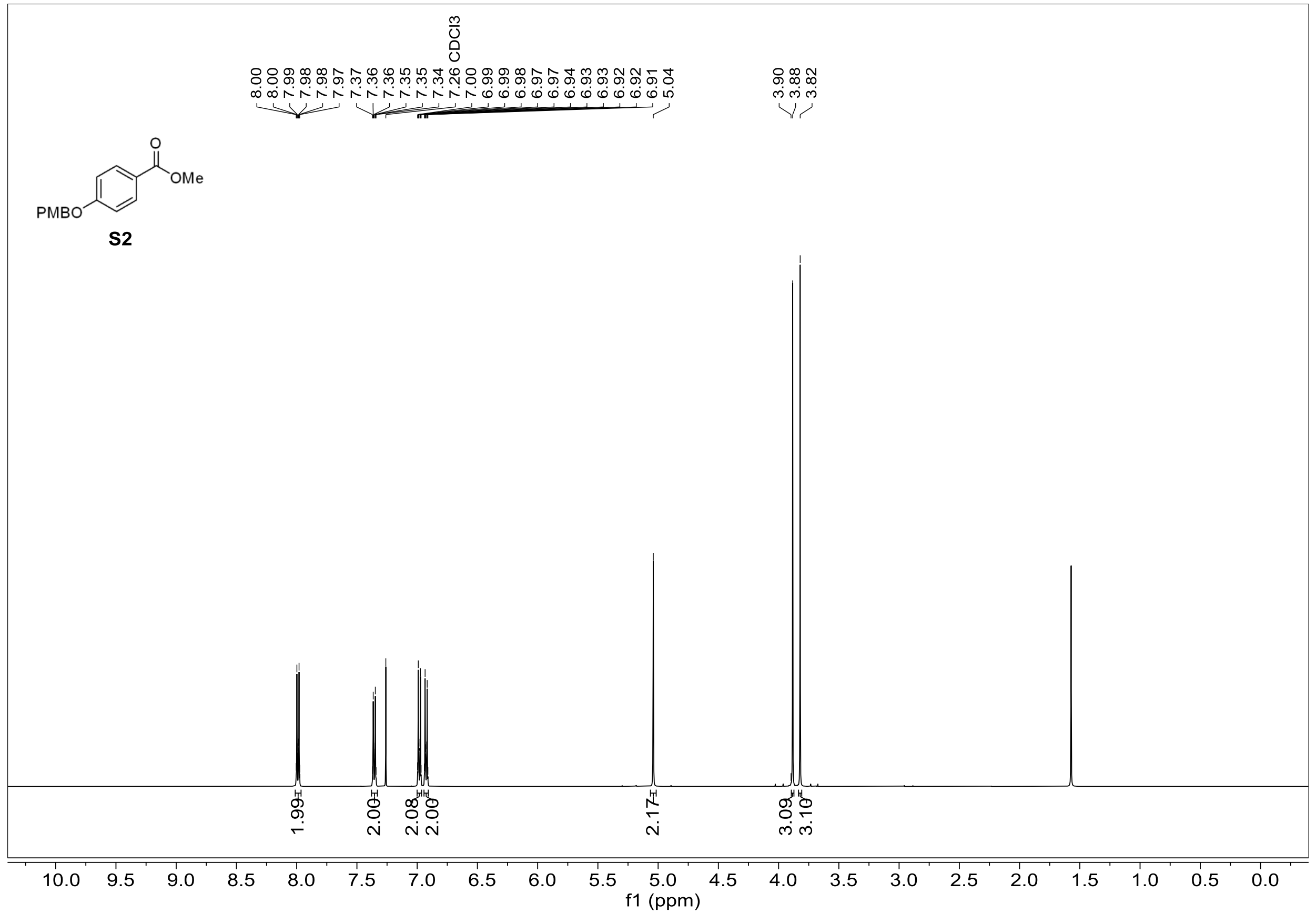

Figure S1. ${ }^{1} \mathrm{H}$ NMR spectrum of $\mathbf{S 2}\left(500 \mathrm{MHz}, \mathrm{CDCl}_{3}\right)$ 


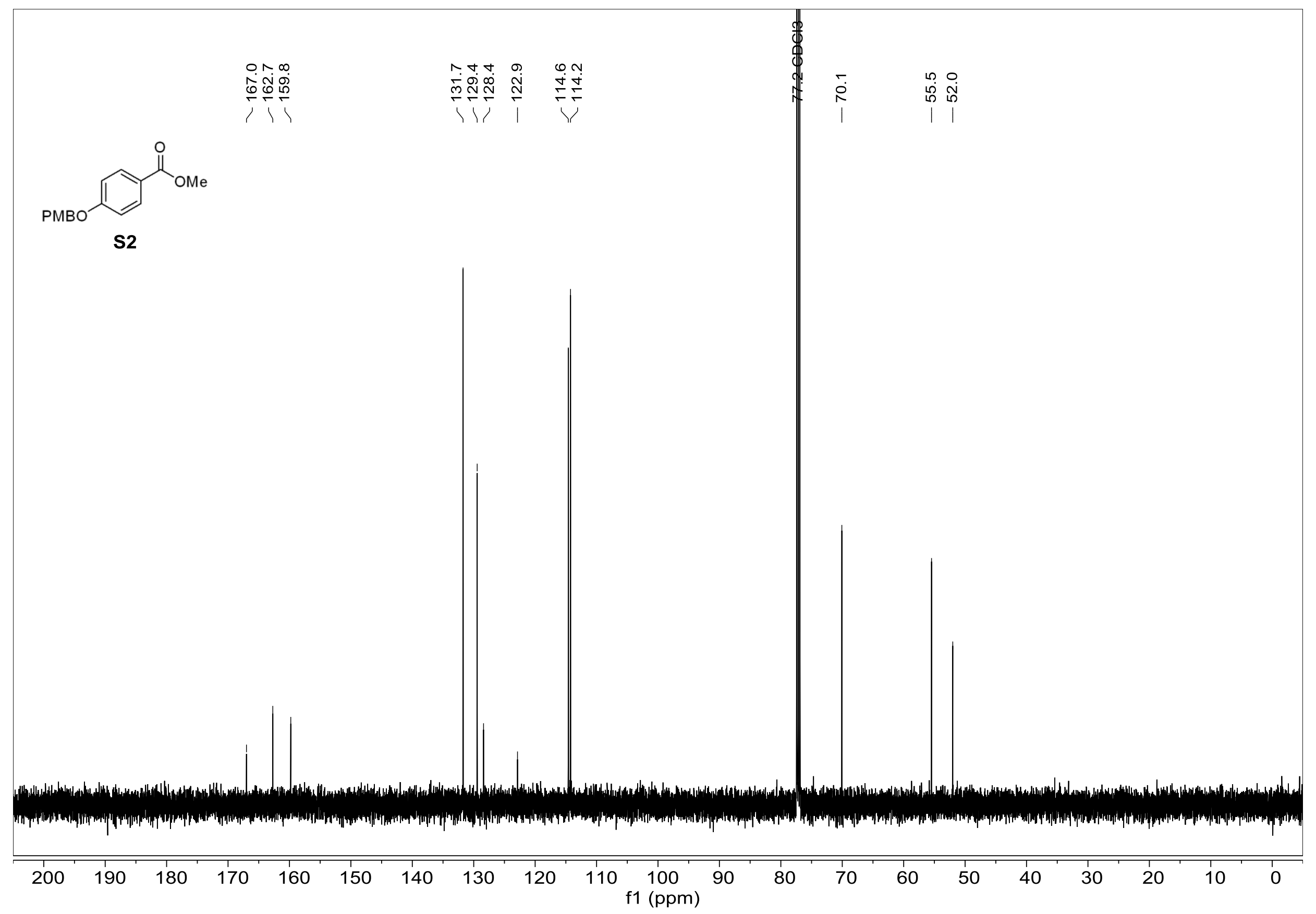

Figure S2. ${ }^{13} \mathrm{C}$ NMR spectrum of $\mathbf{S 2}\left(126 \mathrm{MHz}, \mathrm{CDCl}_{3}\right)$ 


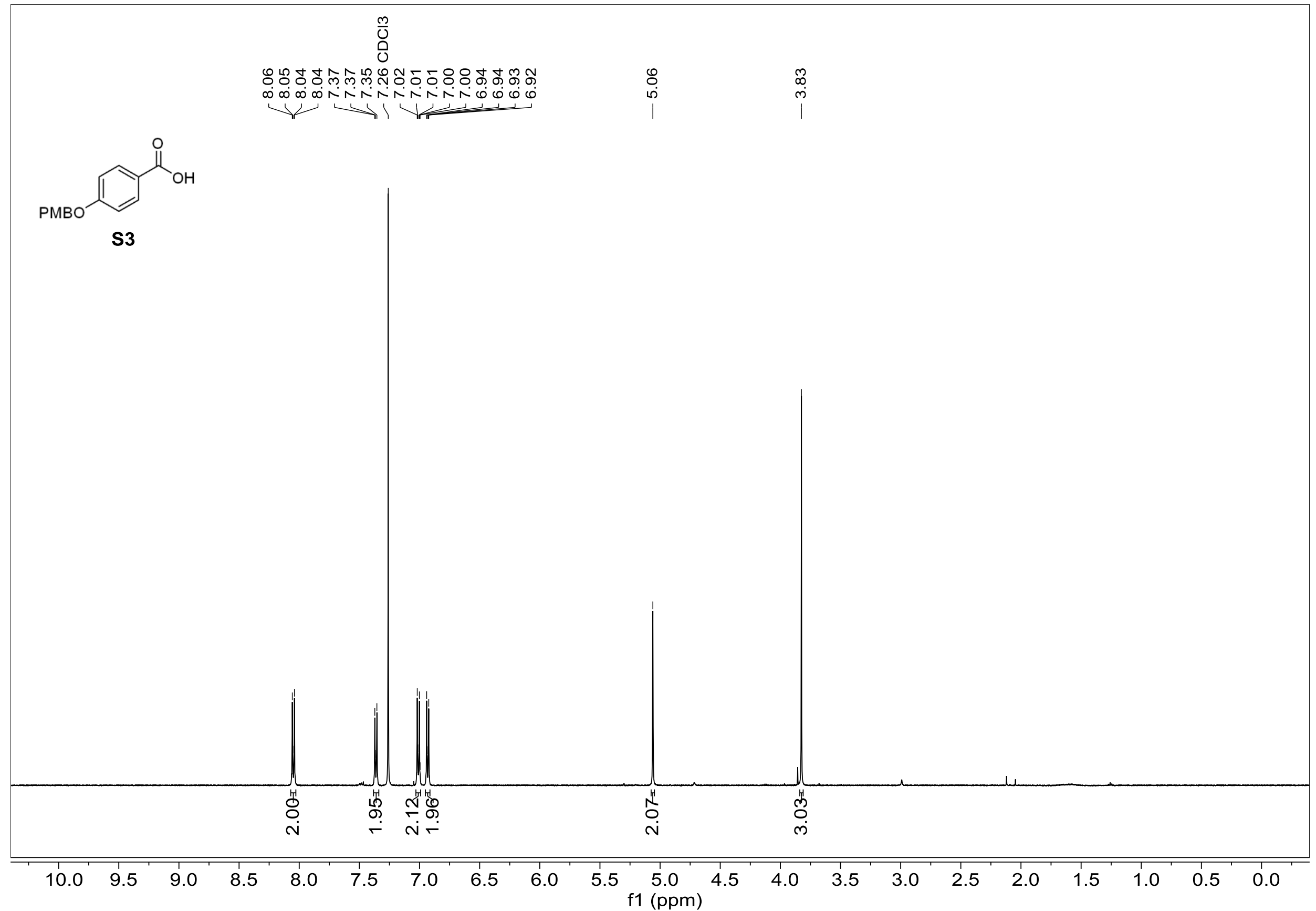

Figure S3. ${ }^{1} \mathrm{H}$ NMR spectrum of $\mathbf{S 3}\left(500 \mathrm{MHz}, \mathrm{CDCl}_{3}\right)$ 


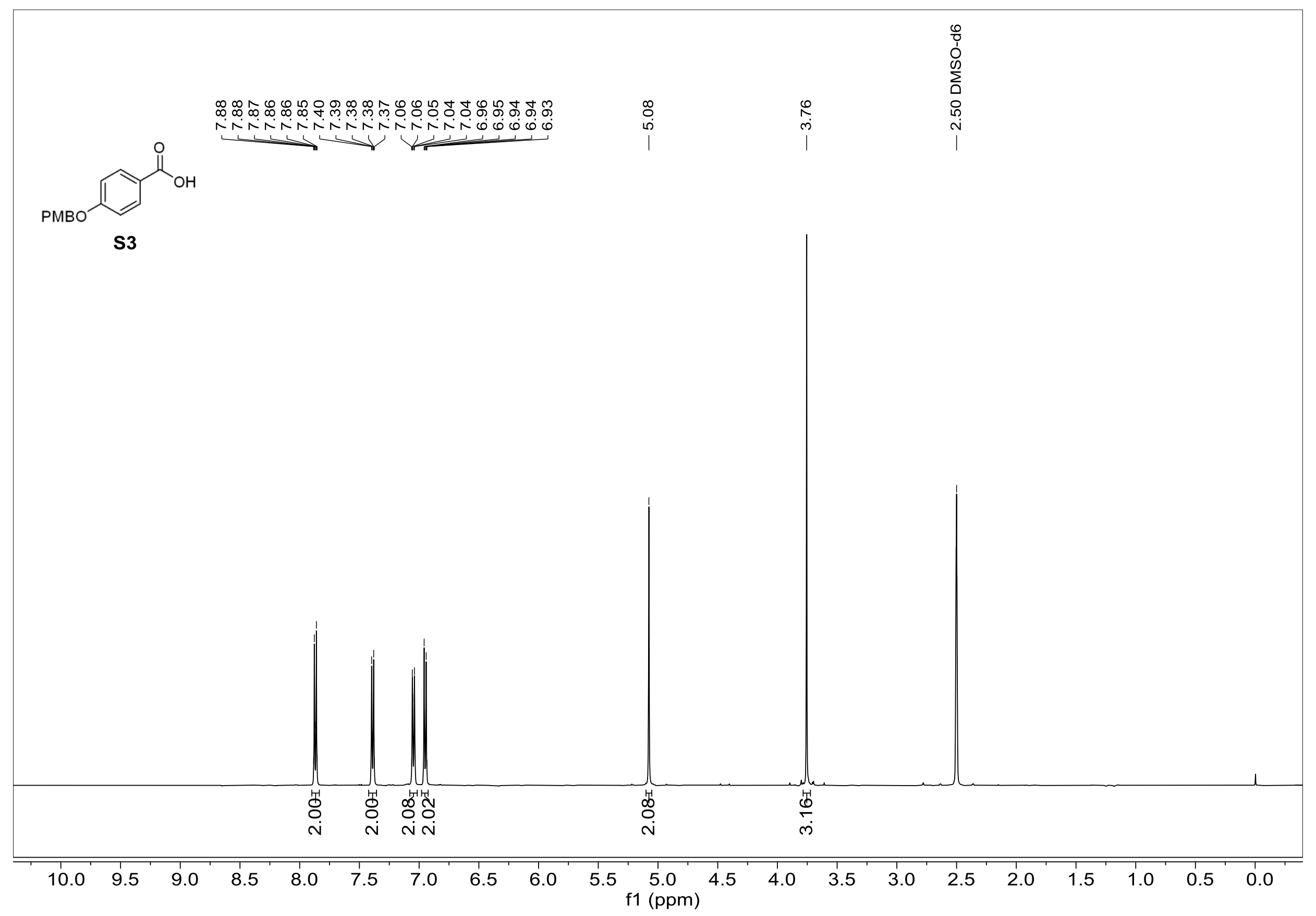

Figure S4. ${ }^{1} \mathrm{H}$ NMR spectrum of $\mathbf{S 3}\left(500 \mathrm{MHz}, \mathrm{DMSO}-d_{6}\right)$ 


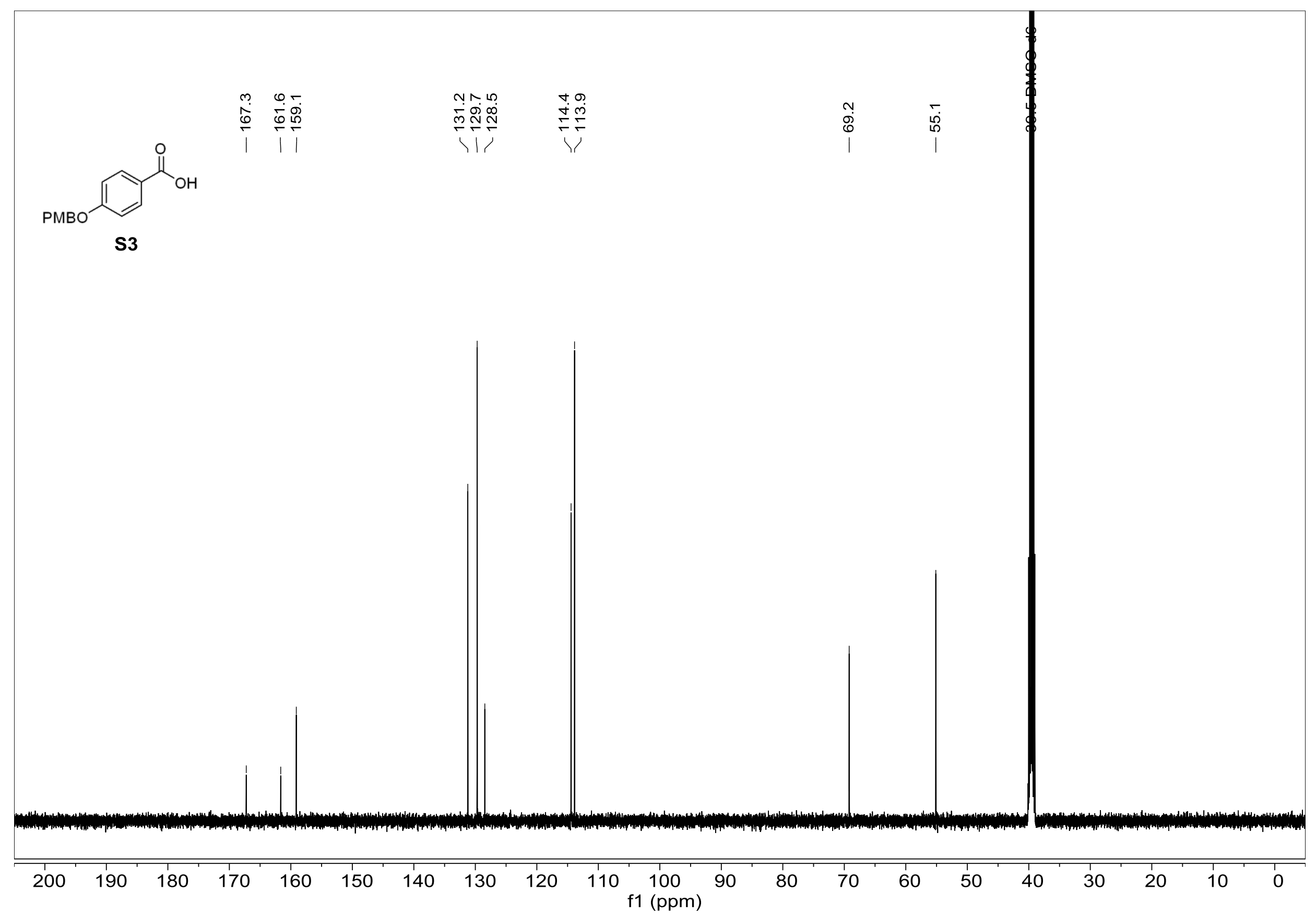

Figure S5. ${ }^{13} \mathrm{C}$ NMR spectrum of $\mathbf{S 3}$ (126 MHz, DMSO- $d_{6}$ ) 


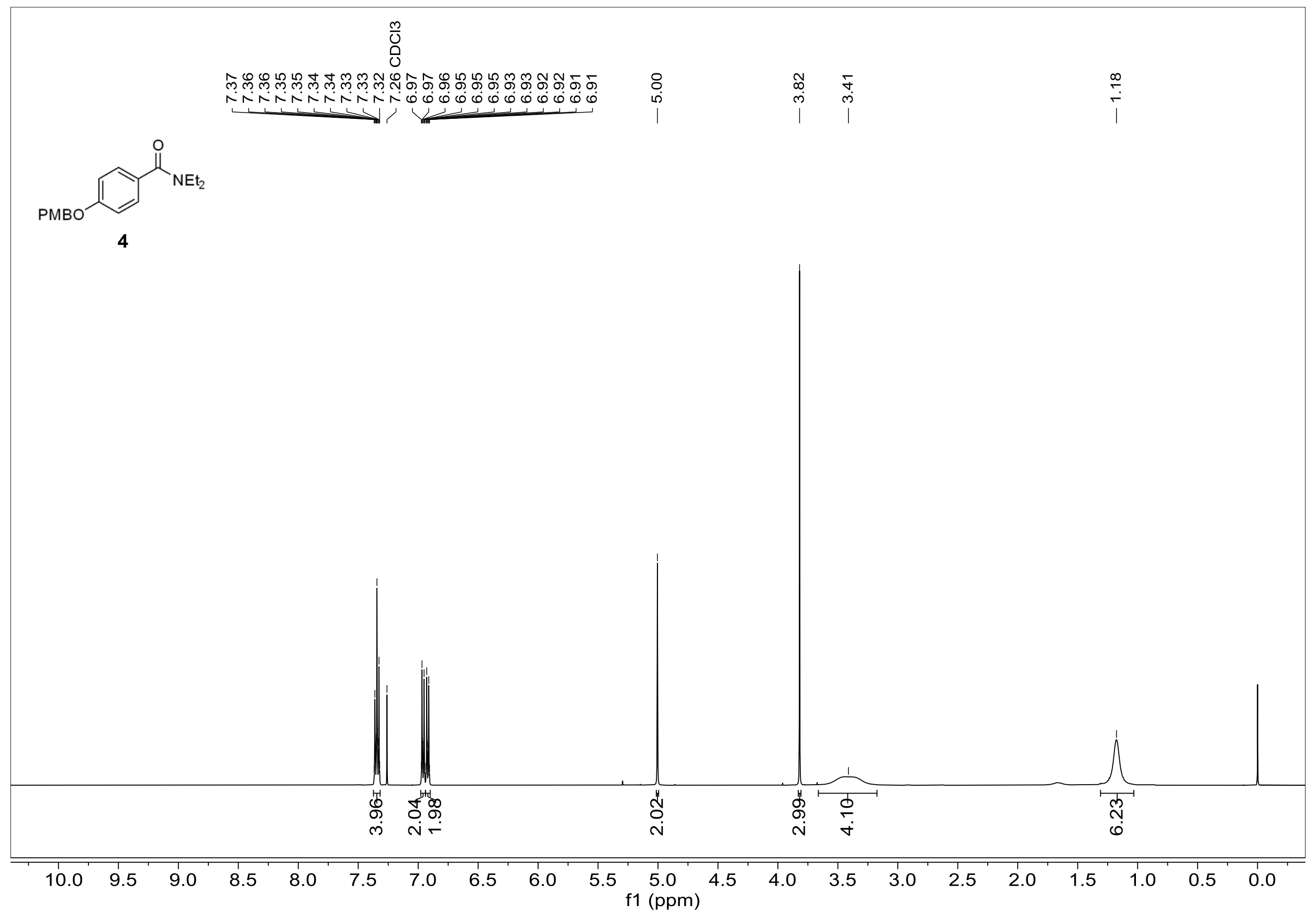

Figure S6. ${ }^{1} \mathrm{H}$ NMR spectrum of $4\left(500 \mathrm{MHz}, \mathrm{CDCl}_{3}\right)$ 


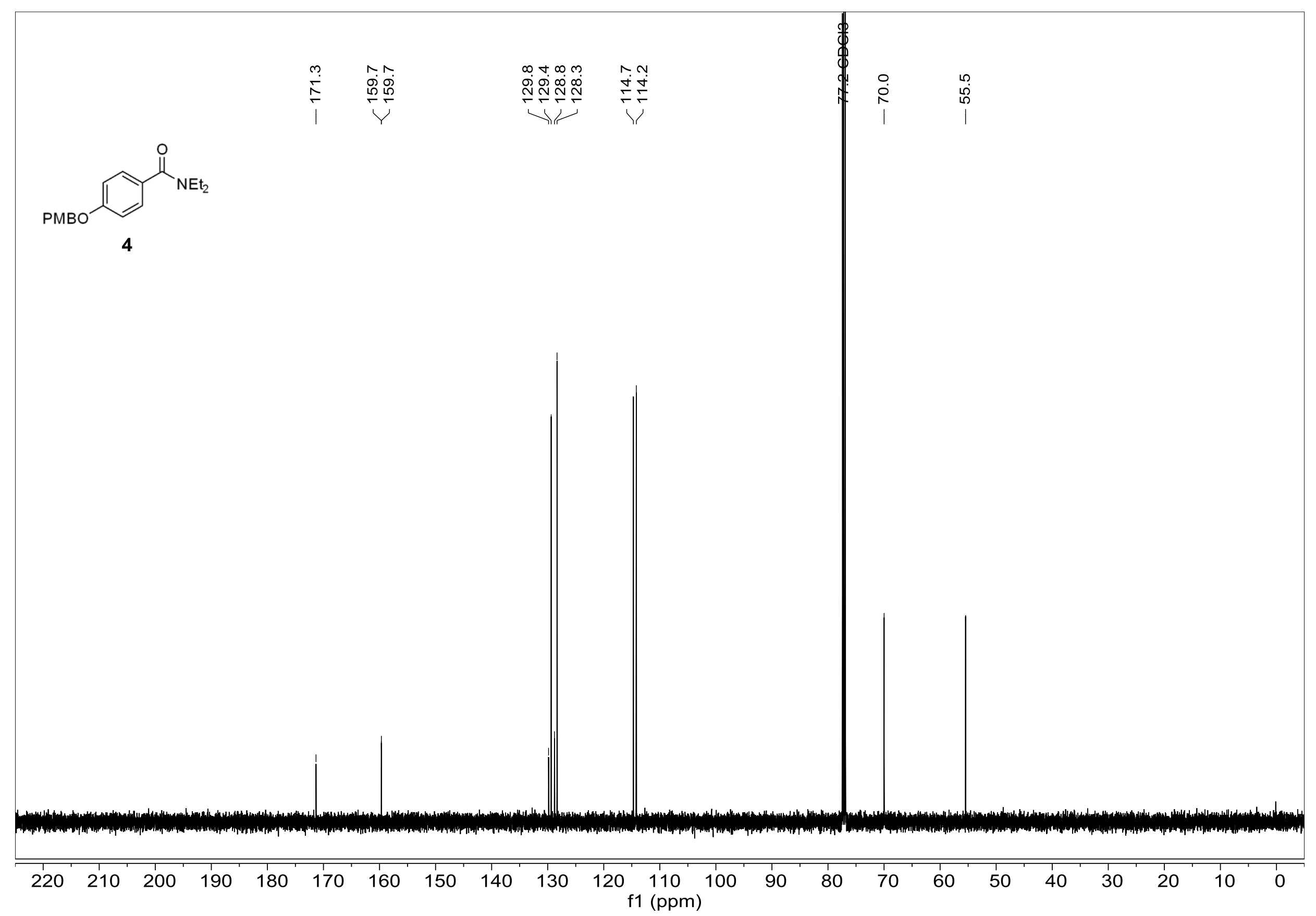

Figure S7. ${ }^{13} \mathrm{C}$ NMR spectrum of $4\left(126 \mathrm{MHz}, \mathrm{CDCl}_{3}\right)$ 


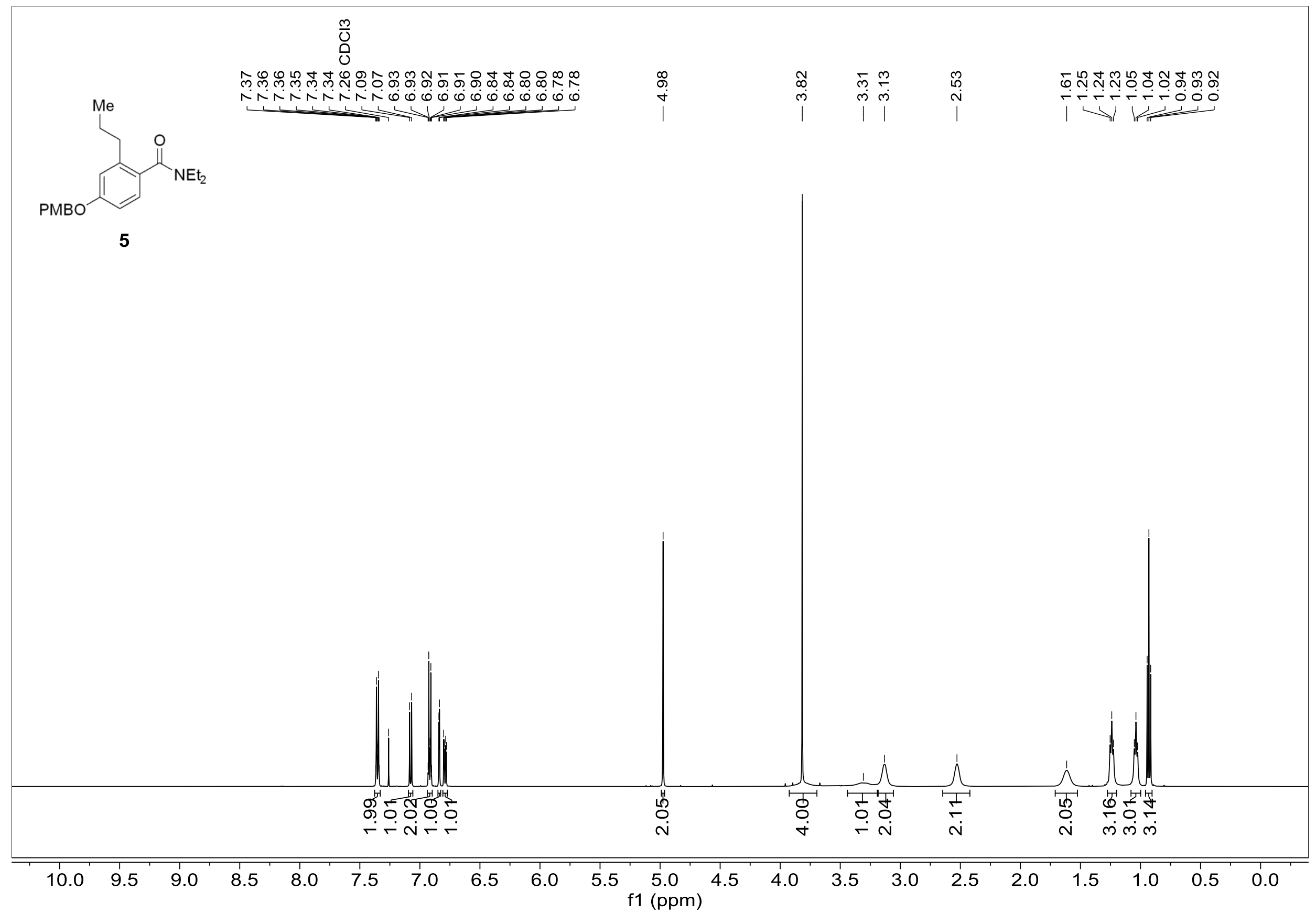

Figure S8. ${ }^{1} \mathrm{H}$ NMR spectrum of $5\left(500 \mathrm{MHz}, \mathrm{CDCl}_{3}\right)$ 


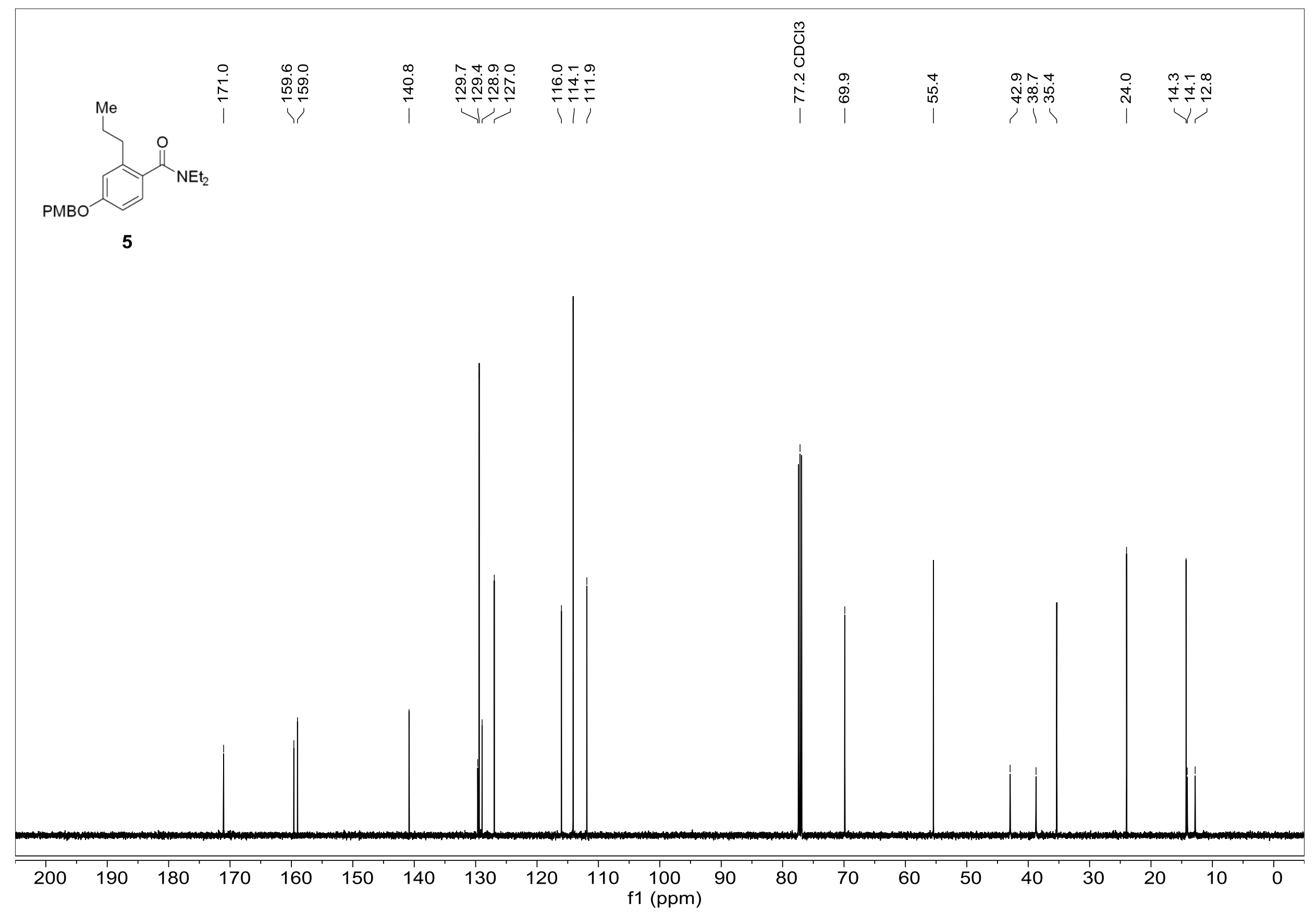

Figure S9. ${ }^{13} \mathrm{C}$ NMR spectrum of $5\left(126 \mathrm{MHz}, \mathrm{CDCl}_{3}\right)$ 


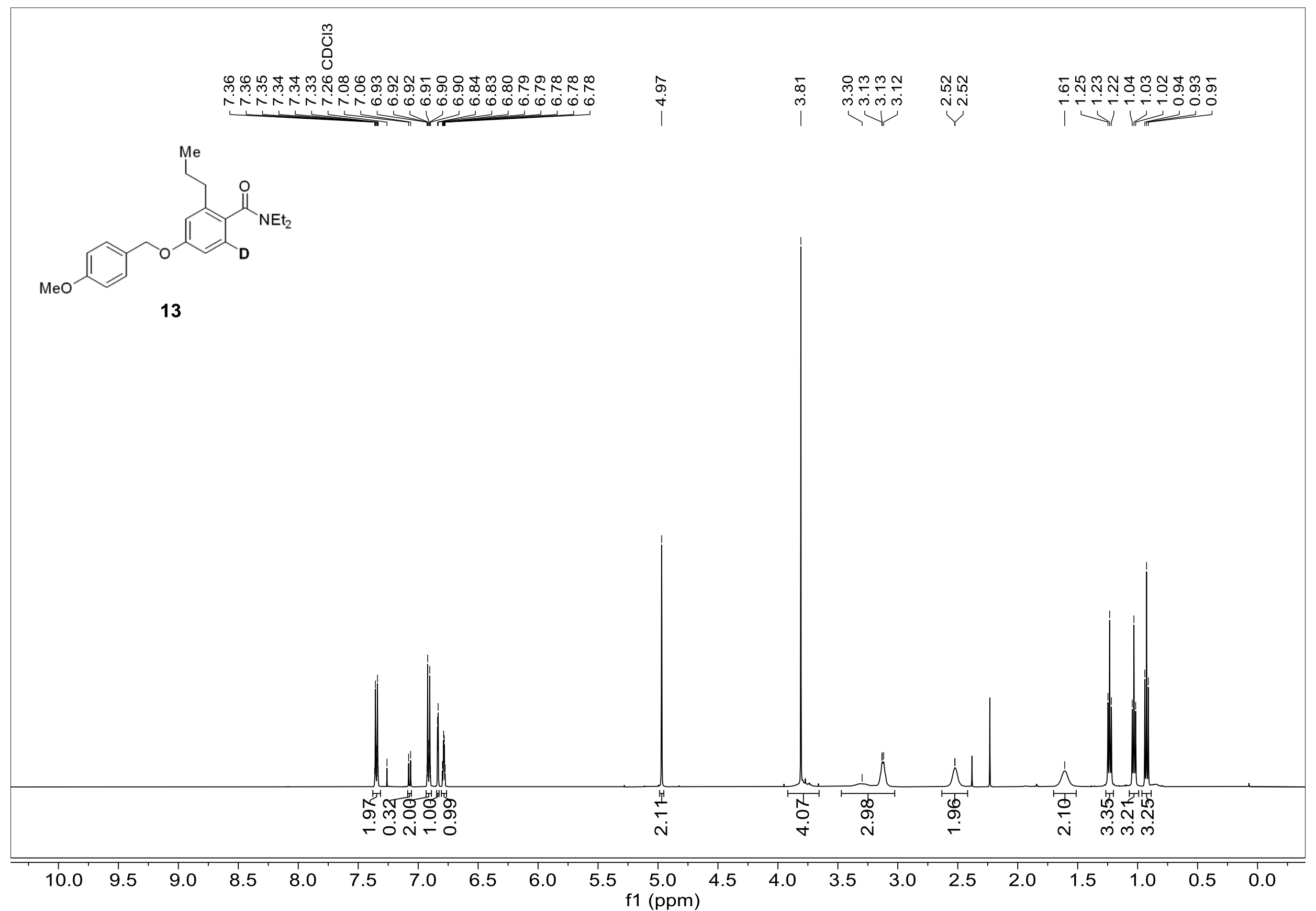

Figure S10. ${ }^{1} \mathrm{H}$ NMR spectrum of $13\left(500 \mathrm{MHz}, \mathrm{CDCl}_{3}\right)$ 


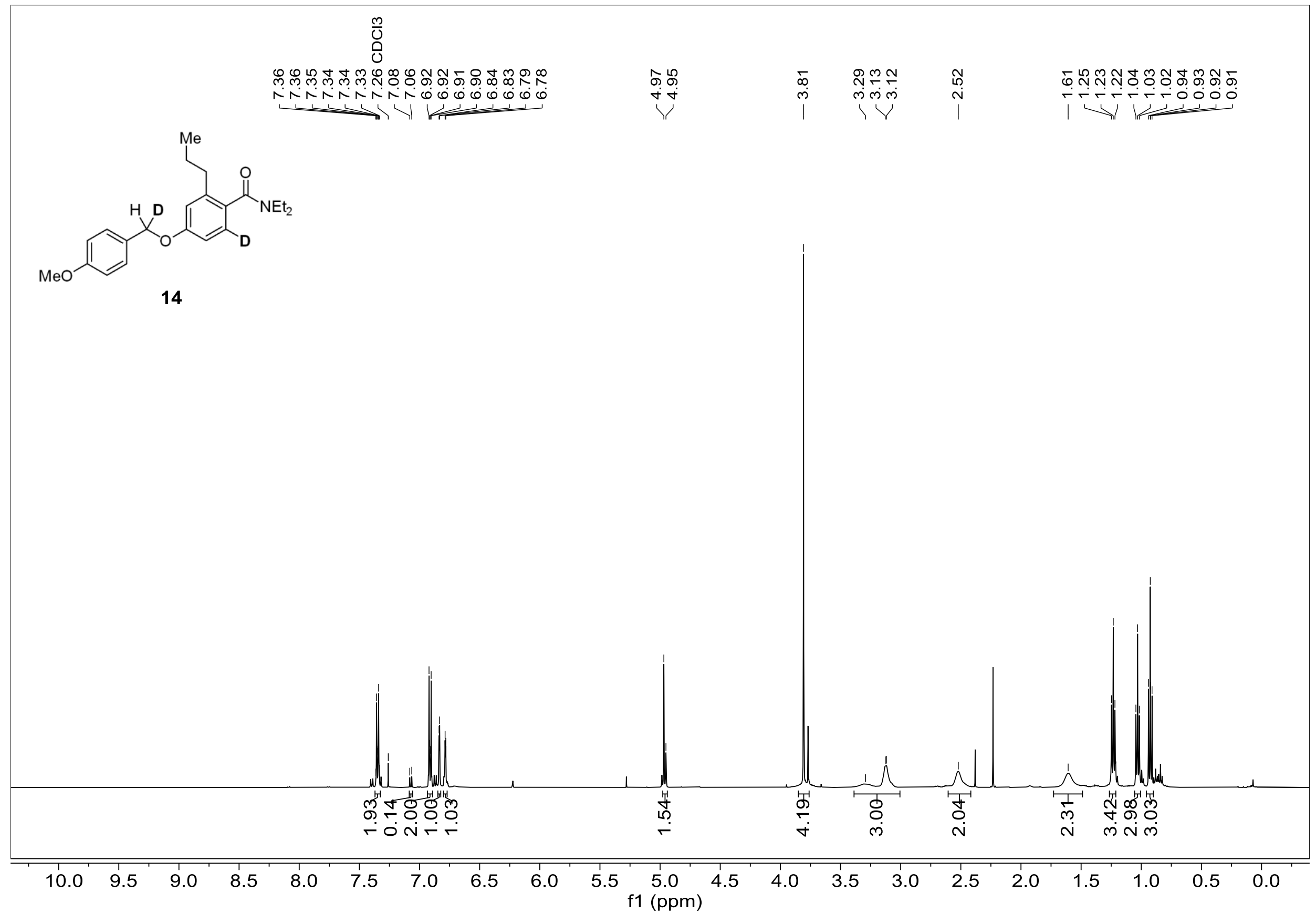

Figure S11. ${ }^{1} \mathrm{H}$ NMR spectrum of $14\left(500 \mathrm{MHz}, \mathrm{CDCl}_{3}\right)$ 


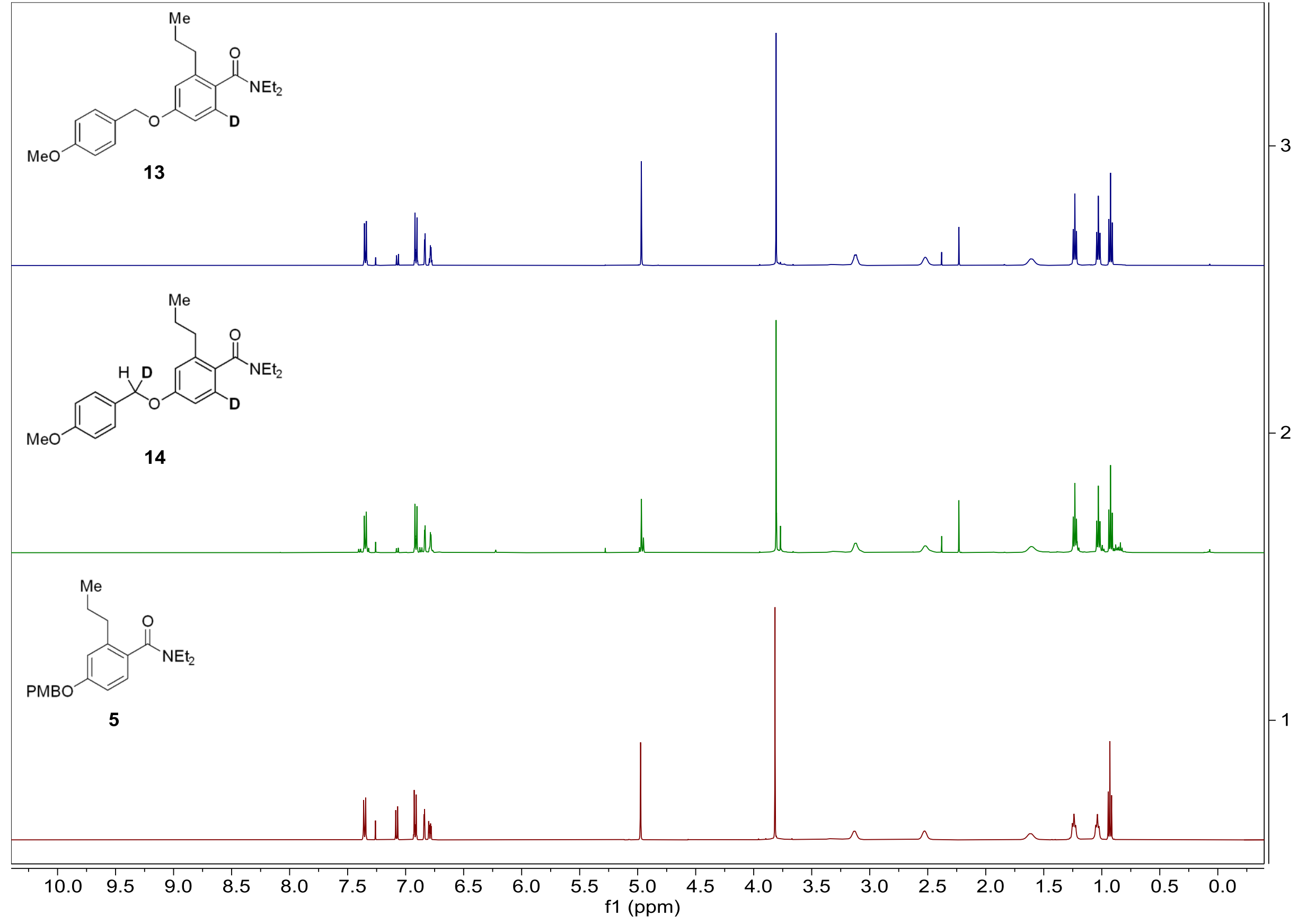

Figure S12. ${ }^{1} \mathrm{H}$ NMR spectrum of $\mathbf{5 , 1 3}$ and $14\left(500 \mathrm{MHz}, \mathrm{CDCl}_{3}\right)$ 


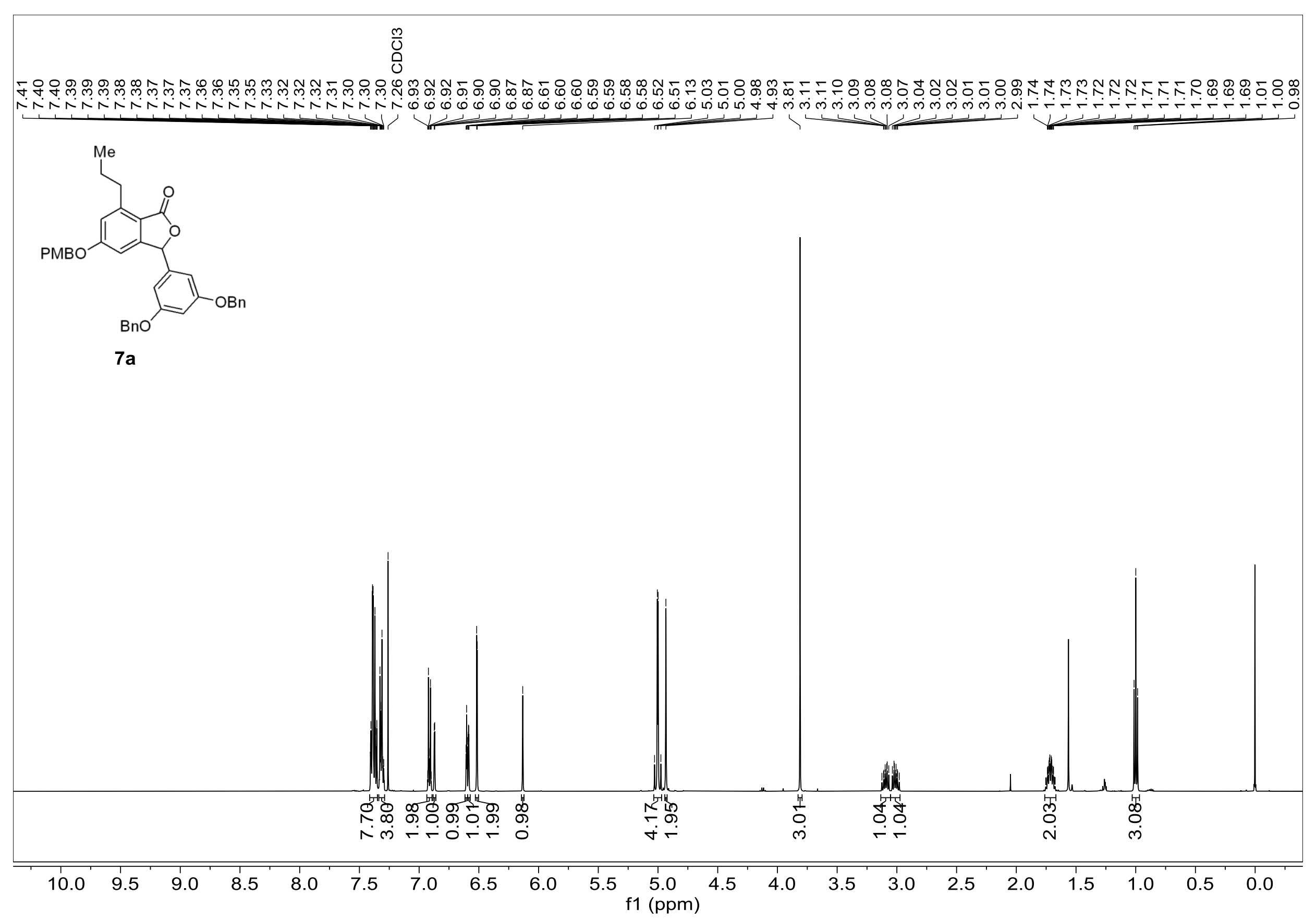

Figure S13. ${ }^{1} \mathrm{H}$ NMR spectrum of $7 \mathbf{a}\left(500 \mathrm{MHz}, \mathrm{CDCl}_{3}\right)$ 


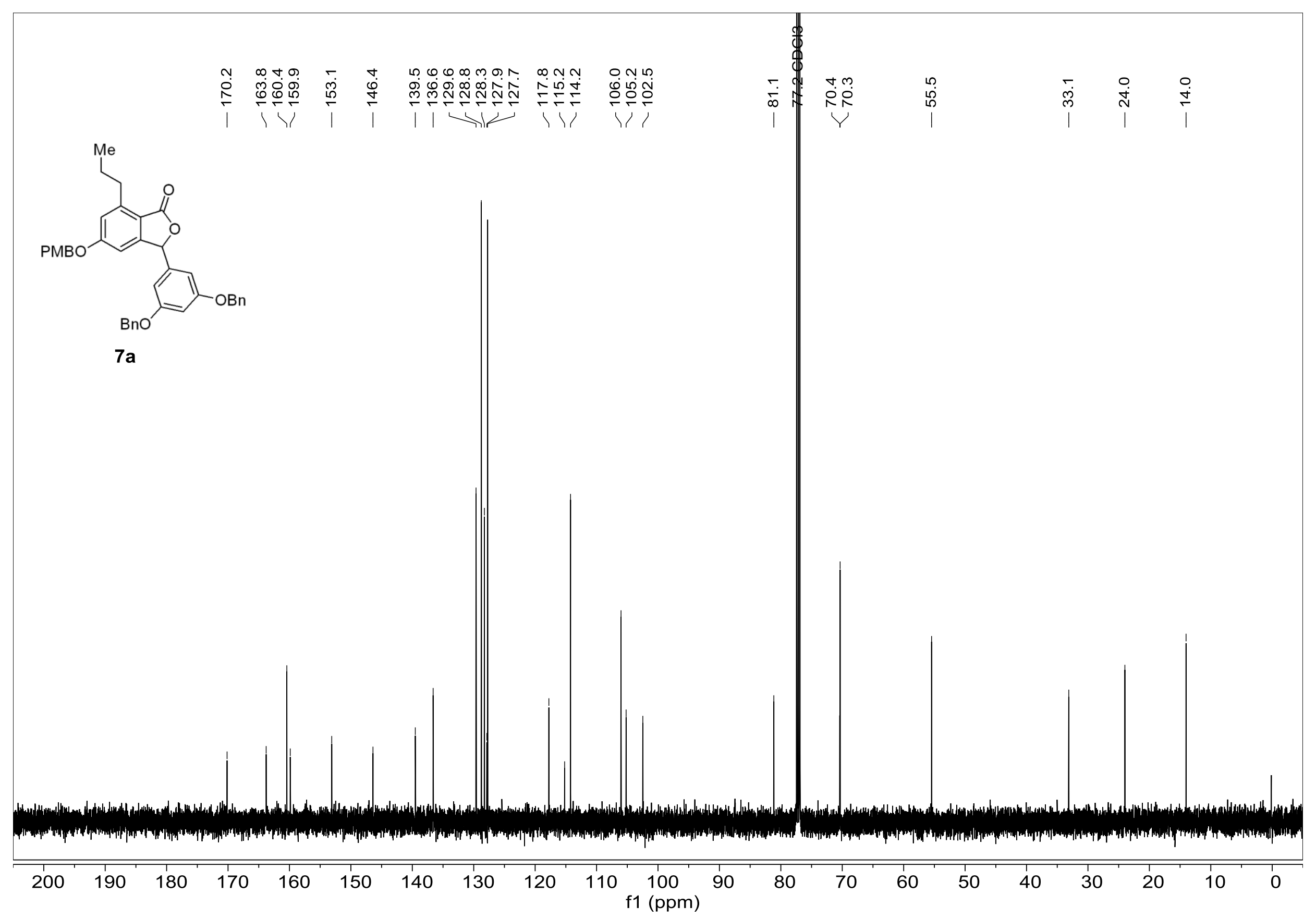

Figure S14. ${ }^{13} \mathrm{C}$ NMR spectrum of $7 \mathbf{a}\left(126 \mathrm{MHz}, \mathrm{CDCl}_{3}\right)$ 


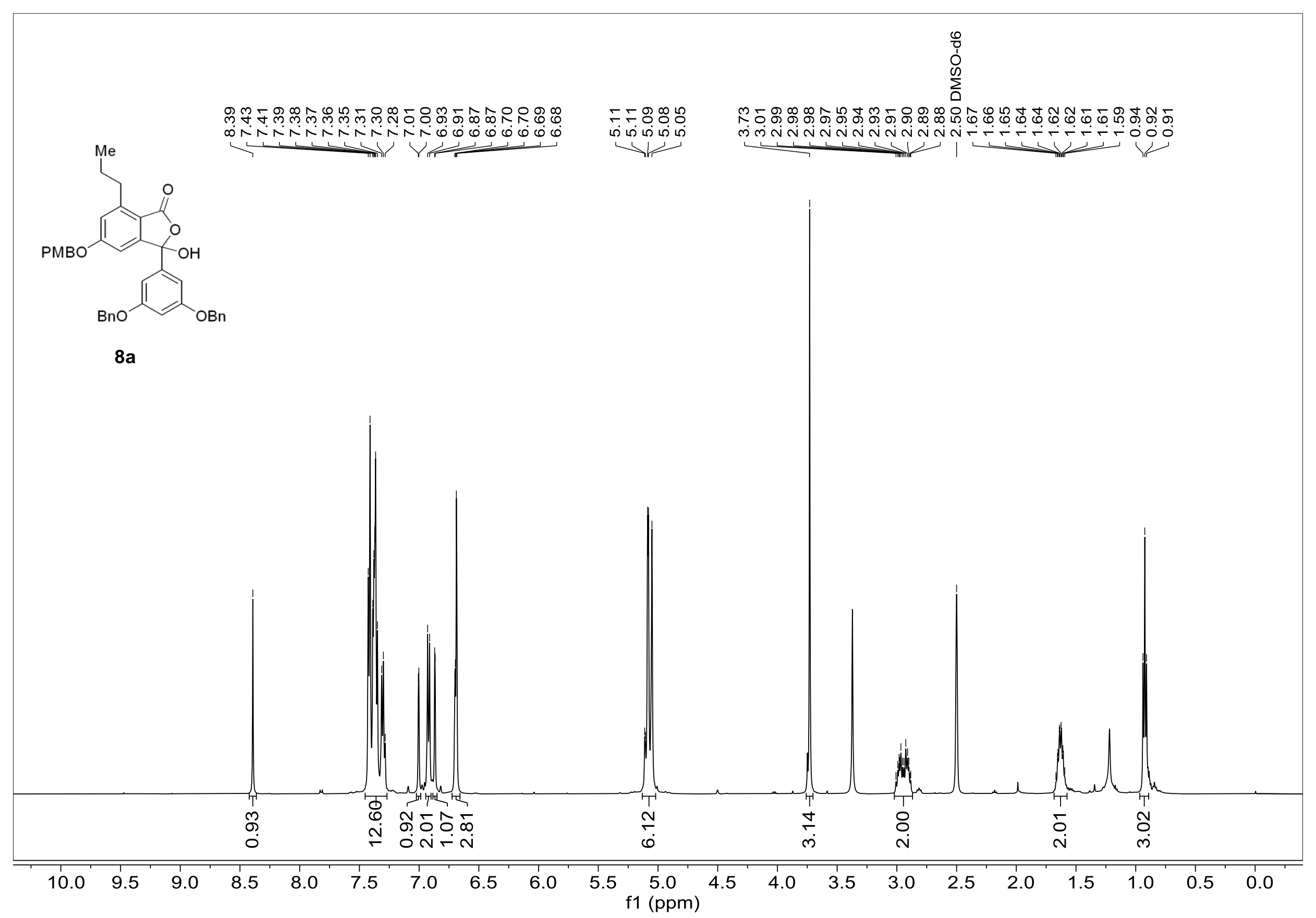

Figure S15. ${ }^{1} \mathrm{H}$ NMR spectrum of $\mathbf{8 a}\left(500 \mathrm{MHz}, \mathrm{DMSO}-d_{6}\right)$ 


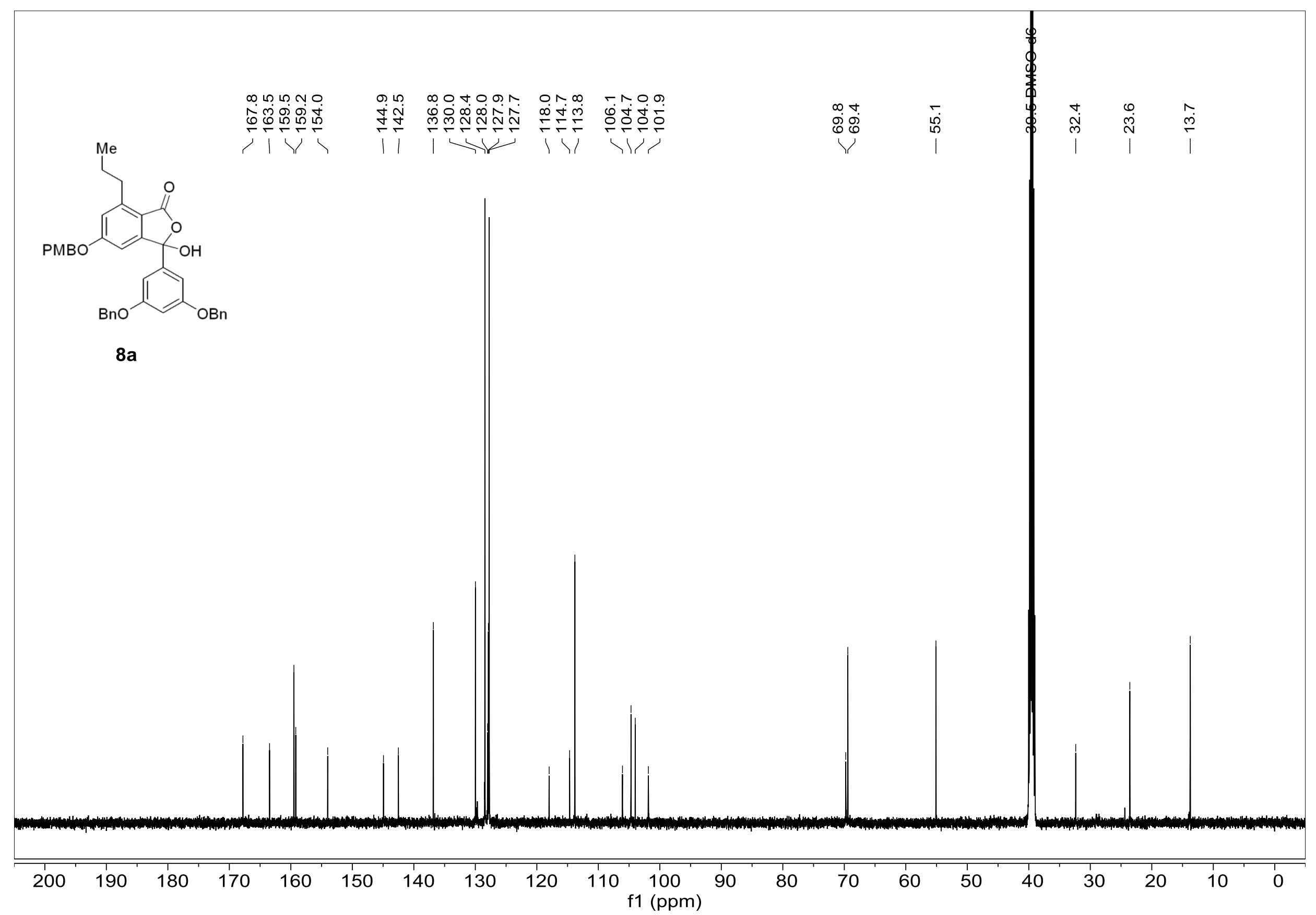

Figure S16. ${ }^{13} \mathrm{C}$ NMR spectrum of $8 \mathbf{a}\left(126 \mathrm{MHz}, \mathrm{DMSO}-d_{6}\right)$ 


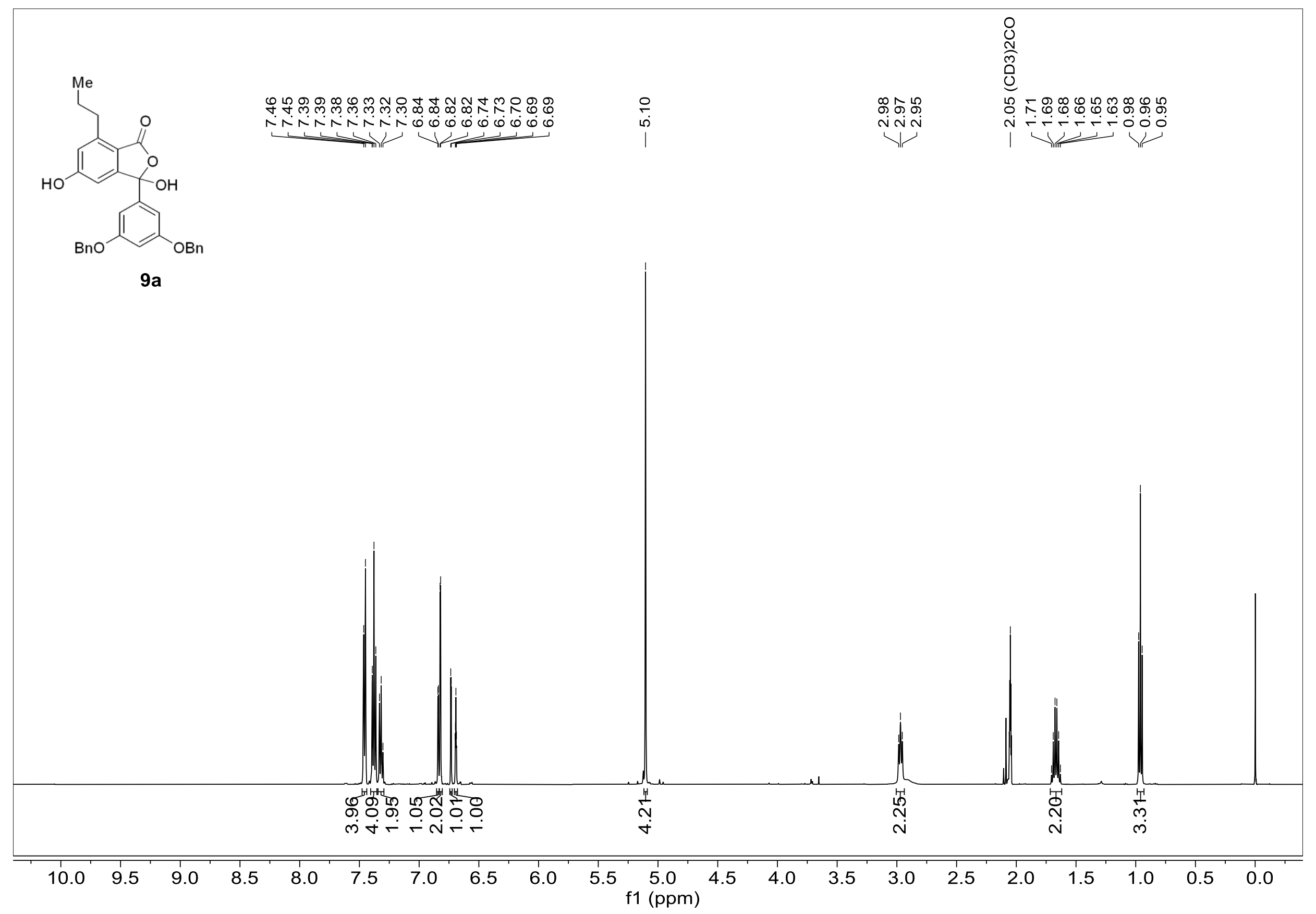

Figure S17. ${ }^{1} \mathrm{H}$ NMR spectrum of $9 \mathbf{a}\left(500 \mathrm{MHz}\right.$, acetone- $\left.d_{6}\right)$ 


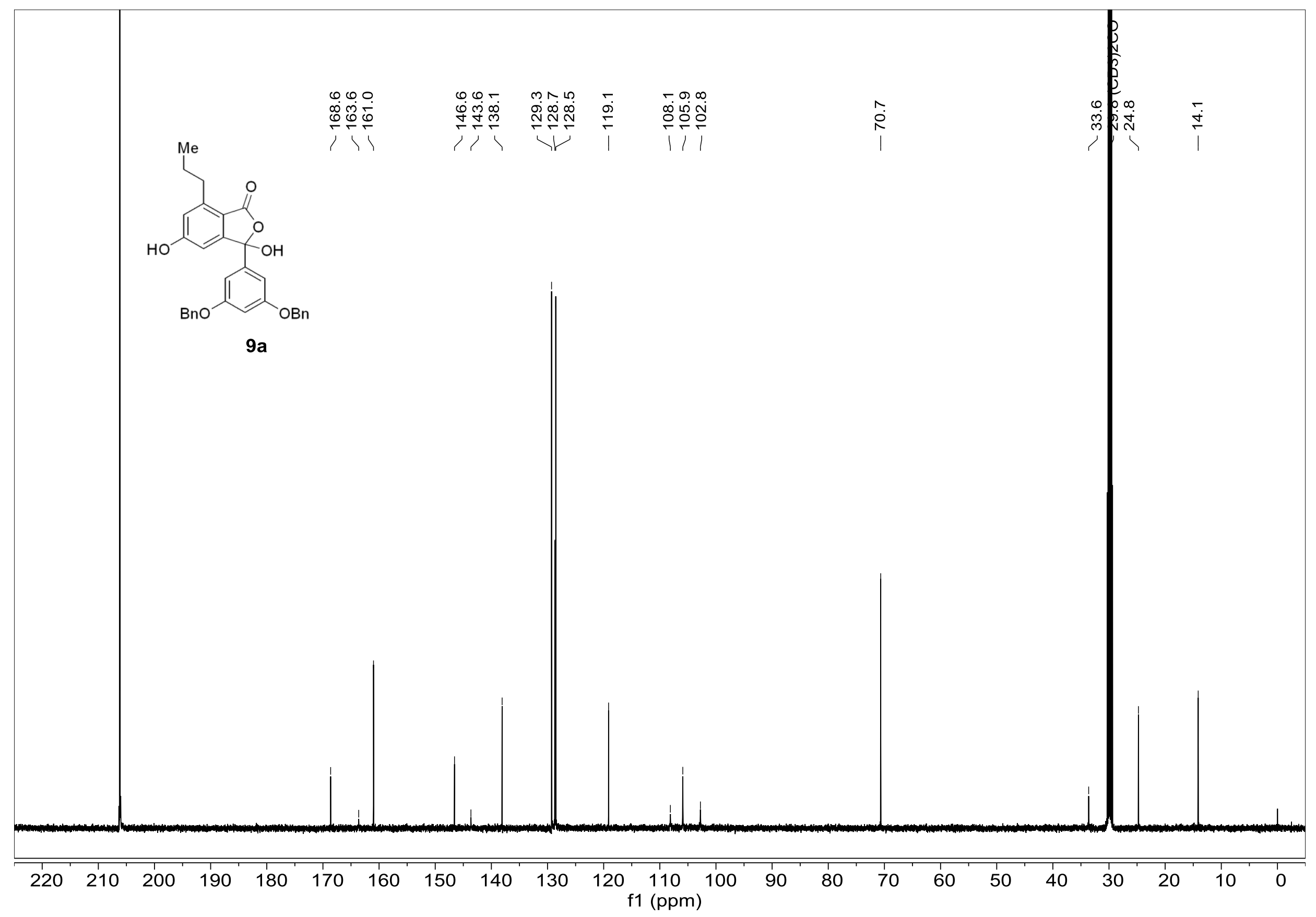

Figure S18. ${ }^{13} \mathrm{C}$ NMR spectrum of 9a (126 MHz, acetone- $d_{6}$ ) 


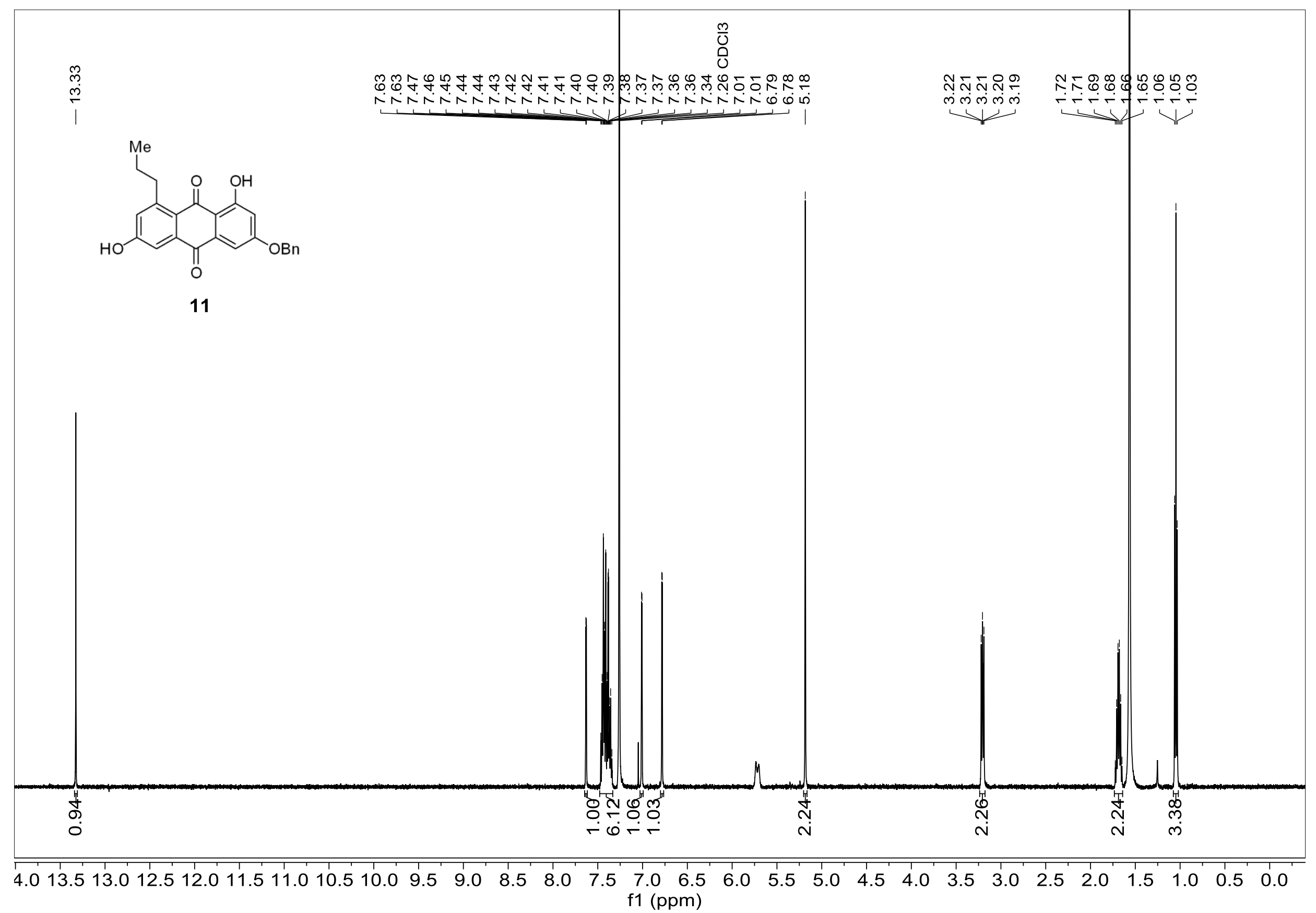

Figure S19. ${ }^{1} \mathrm{H}$ NMR spectrum of $11\left(500 \mathrm{MHz}, \mathrm{CDCl}_{3}\right)$ 


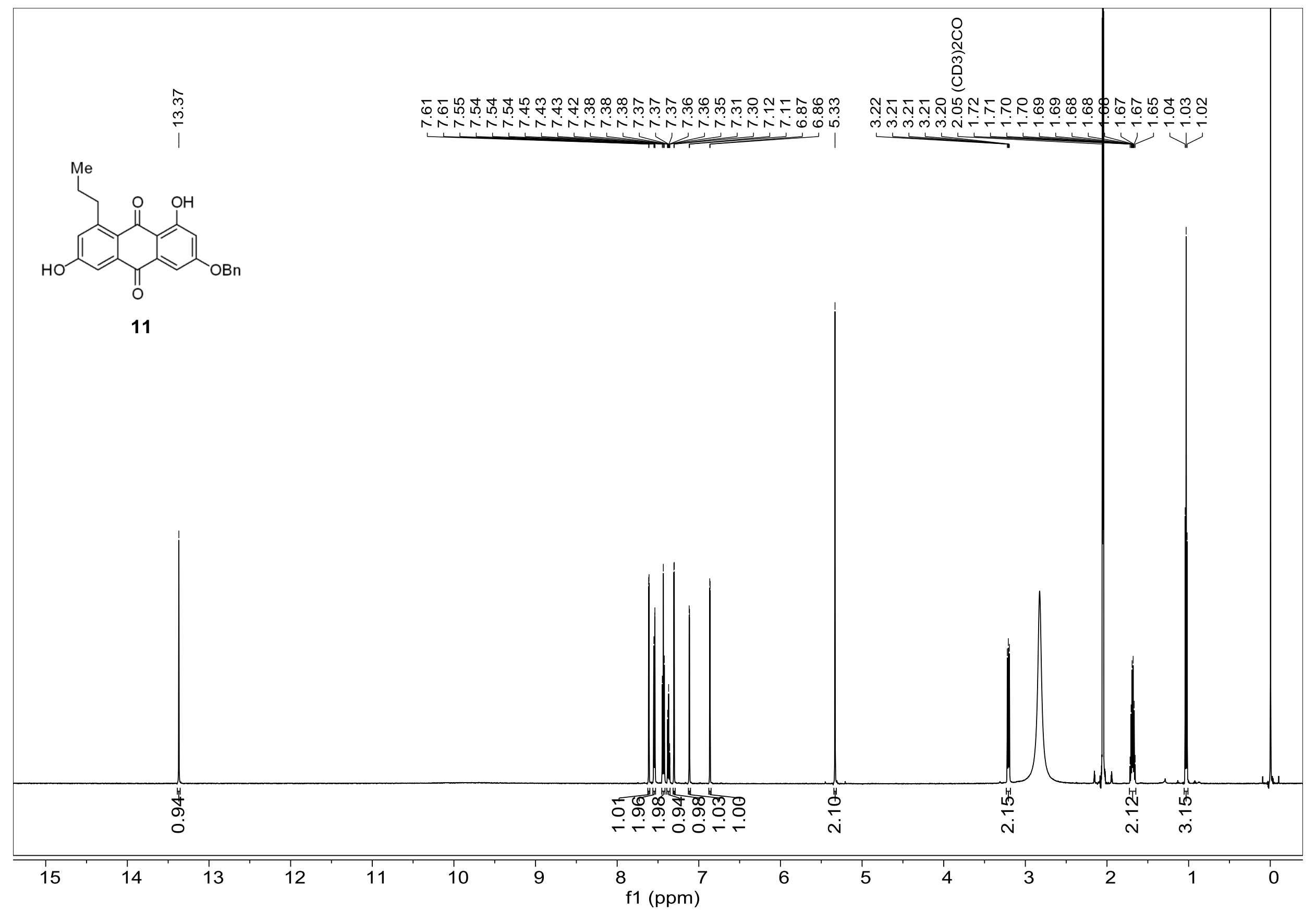

Figure S20. ${ }^{1} \mathrm{H}$ NMR spectrum of $11\left(600 \mathrm{MHz}\right.$, acetone- $\left.d_{6}\right)$ 


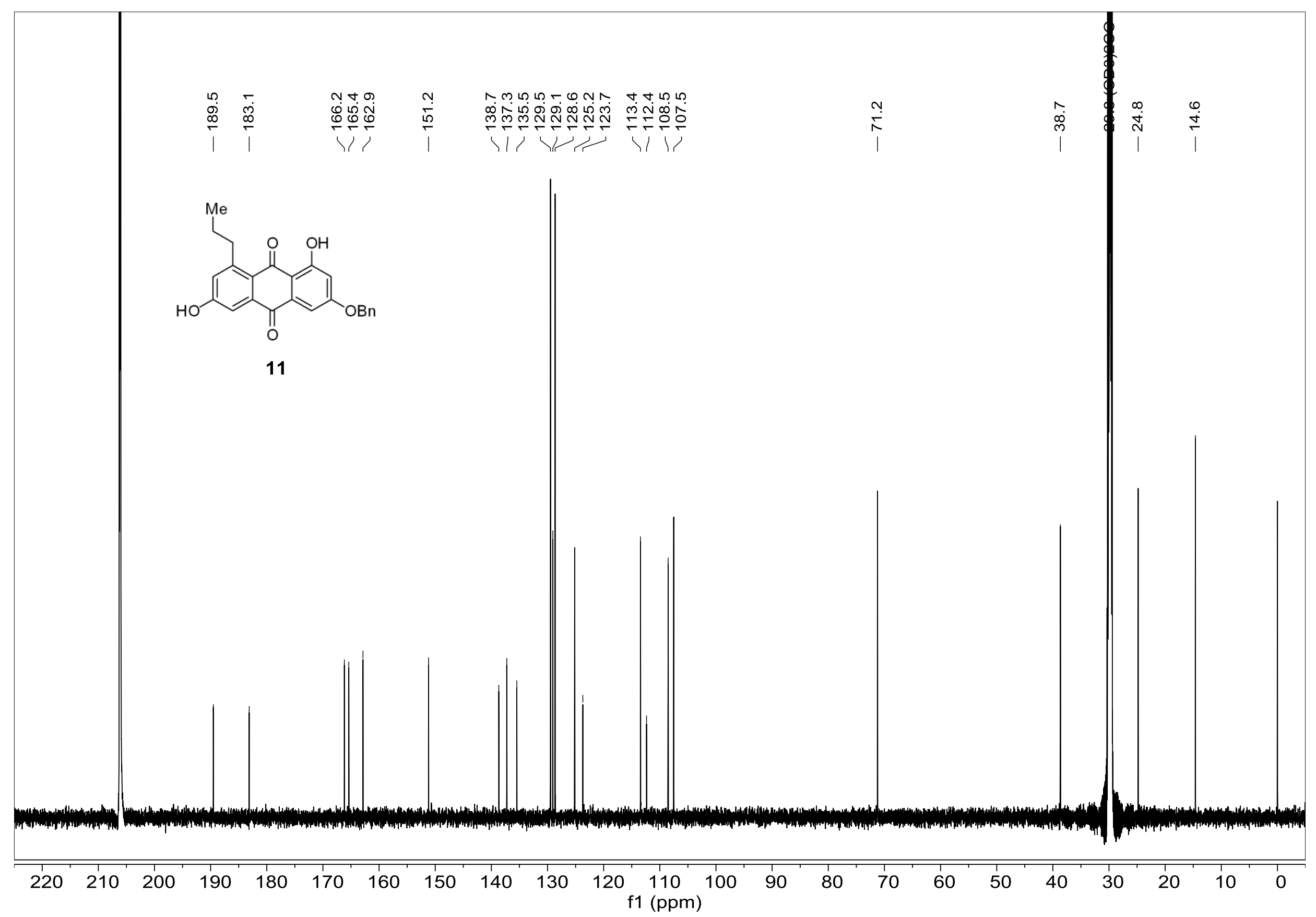

Figure S21. ${ }^{13} \mathrm{C}$ NMR spectrum of 11 (151 MHz, acetone- $d_{6}$ ) 


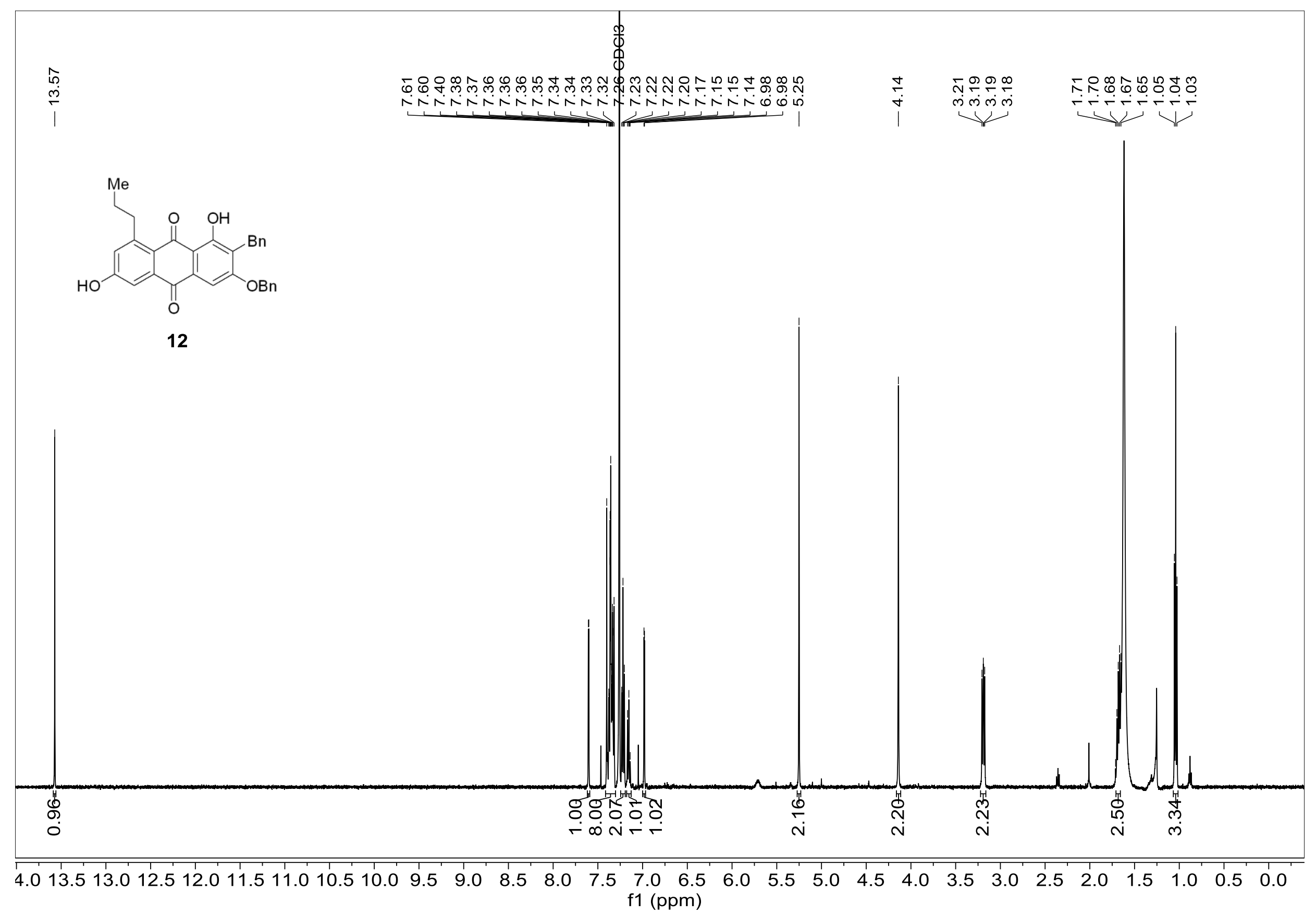

Figure S22. ${ }^{1} \mathrm{H}$ NMR spectrum of $12\left(500 \mathrm{MHz}, \mathrm{CDCl}_{3}\right)$ 


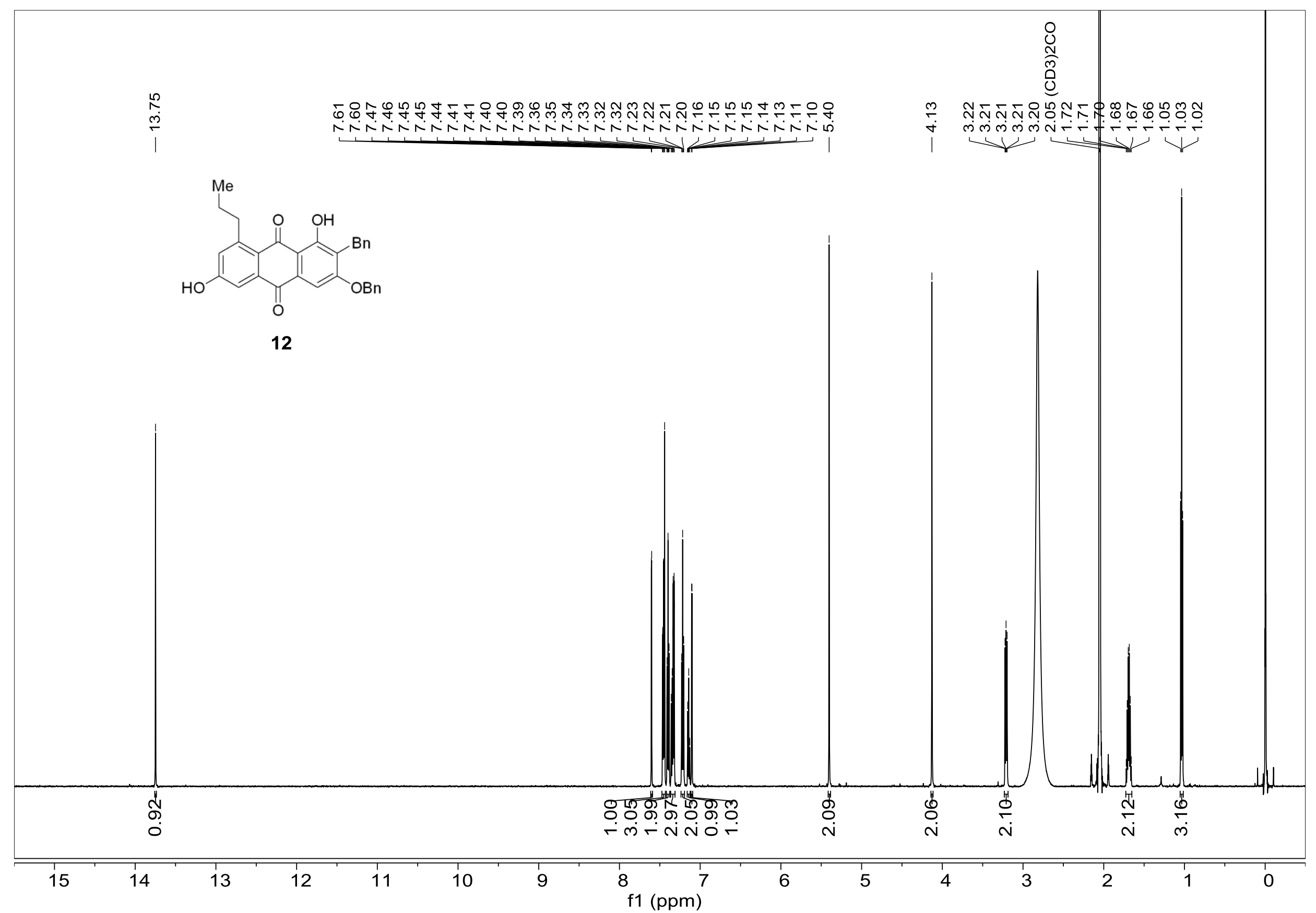

Figure S23. ${ }^{1} \mathrm{H}$ NMR spectrum of $12\left(600 \mathrm{MHz}\right.$, acetone- $\left.d_{6}\right)$ 


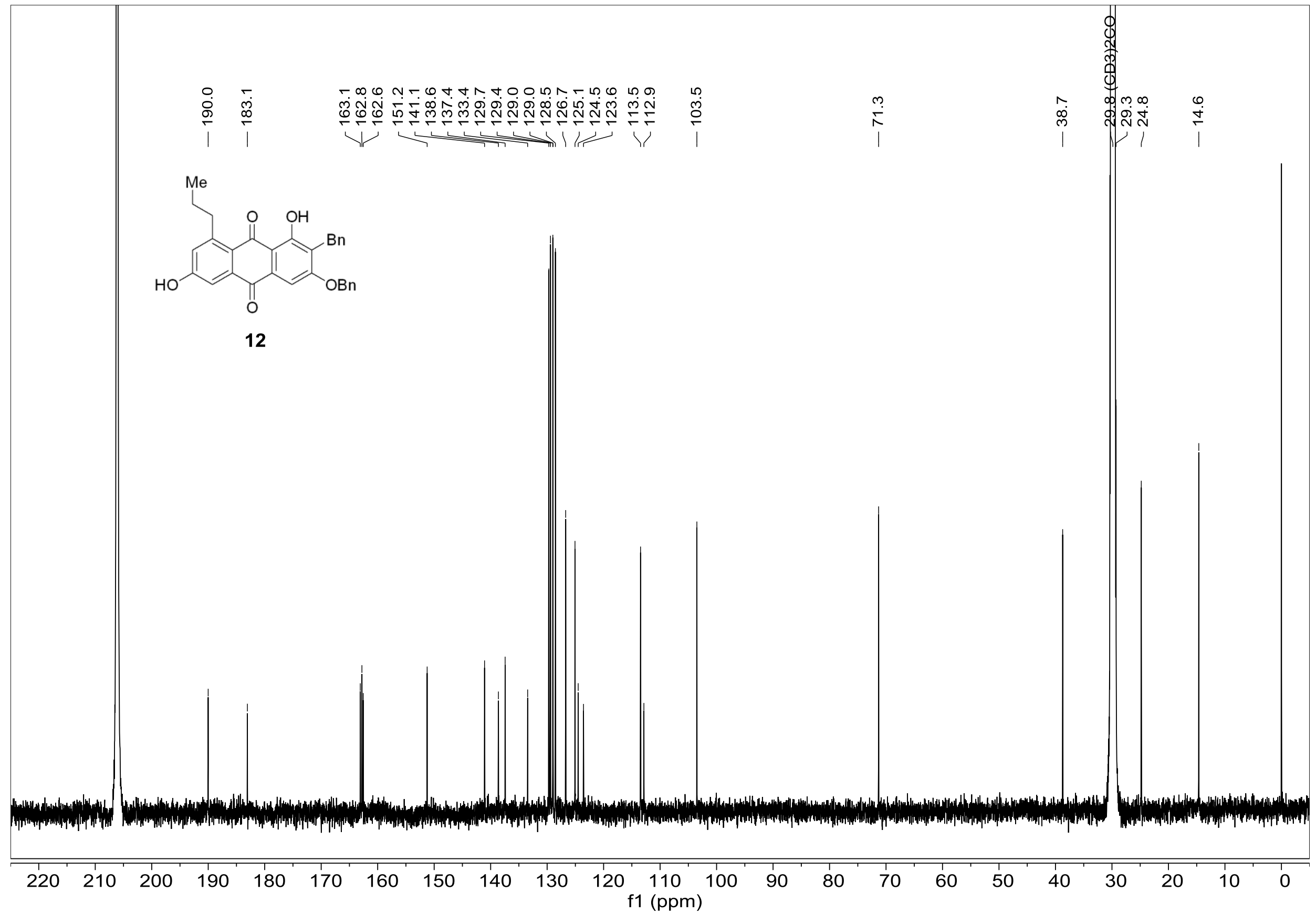

Figure S24. ${ }^{13} \mathrm{C}$ NMR spectrum of $12\left(151 \mathrm{MHz}\right.$, acetone- $\left.d_{6}\right)$ 


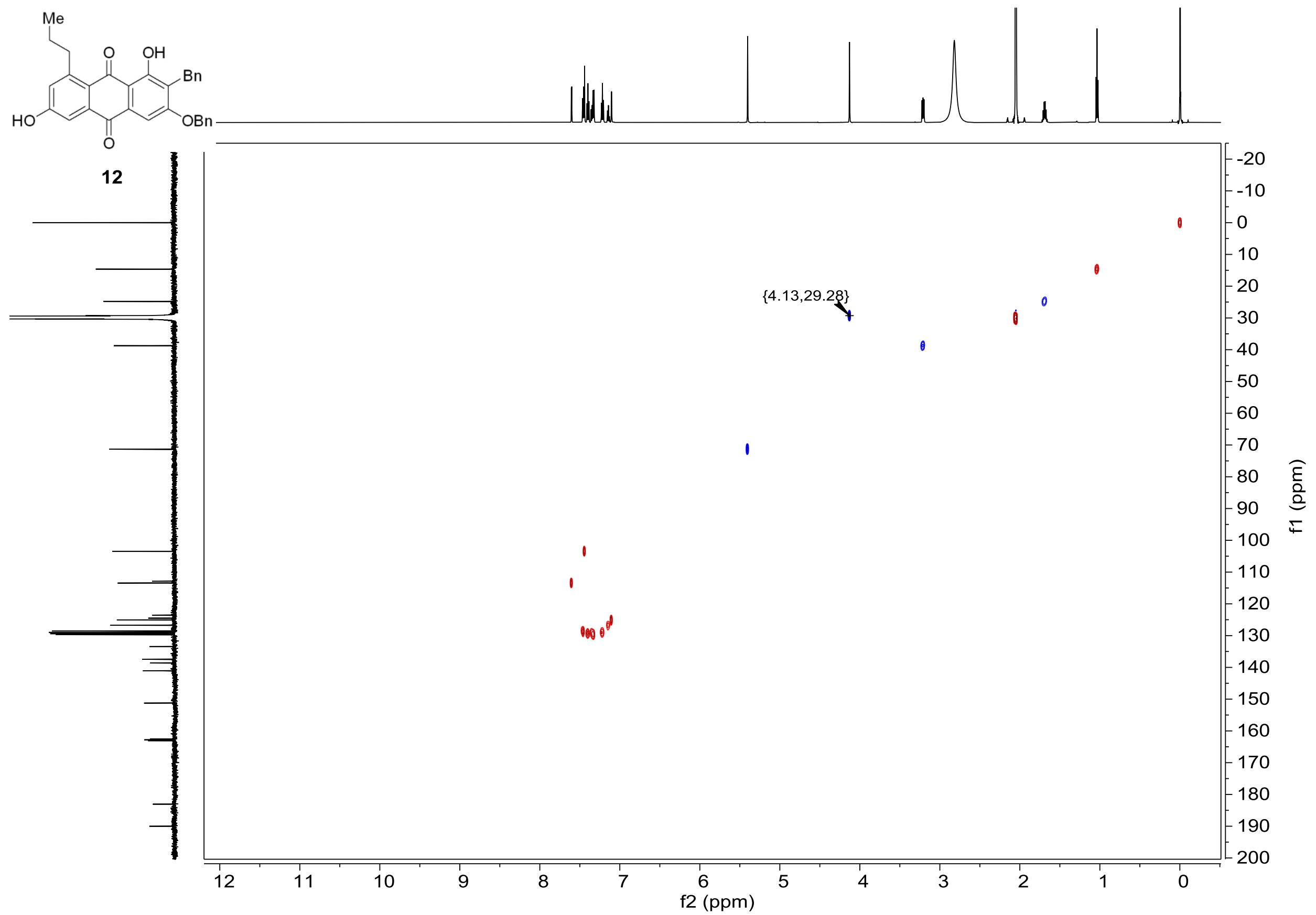

Figure S25. HSQC spectrum of $\mathbf{1 2}$ in acetone- $d_{6}$ 


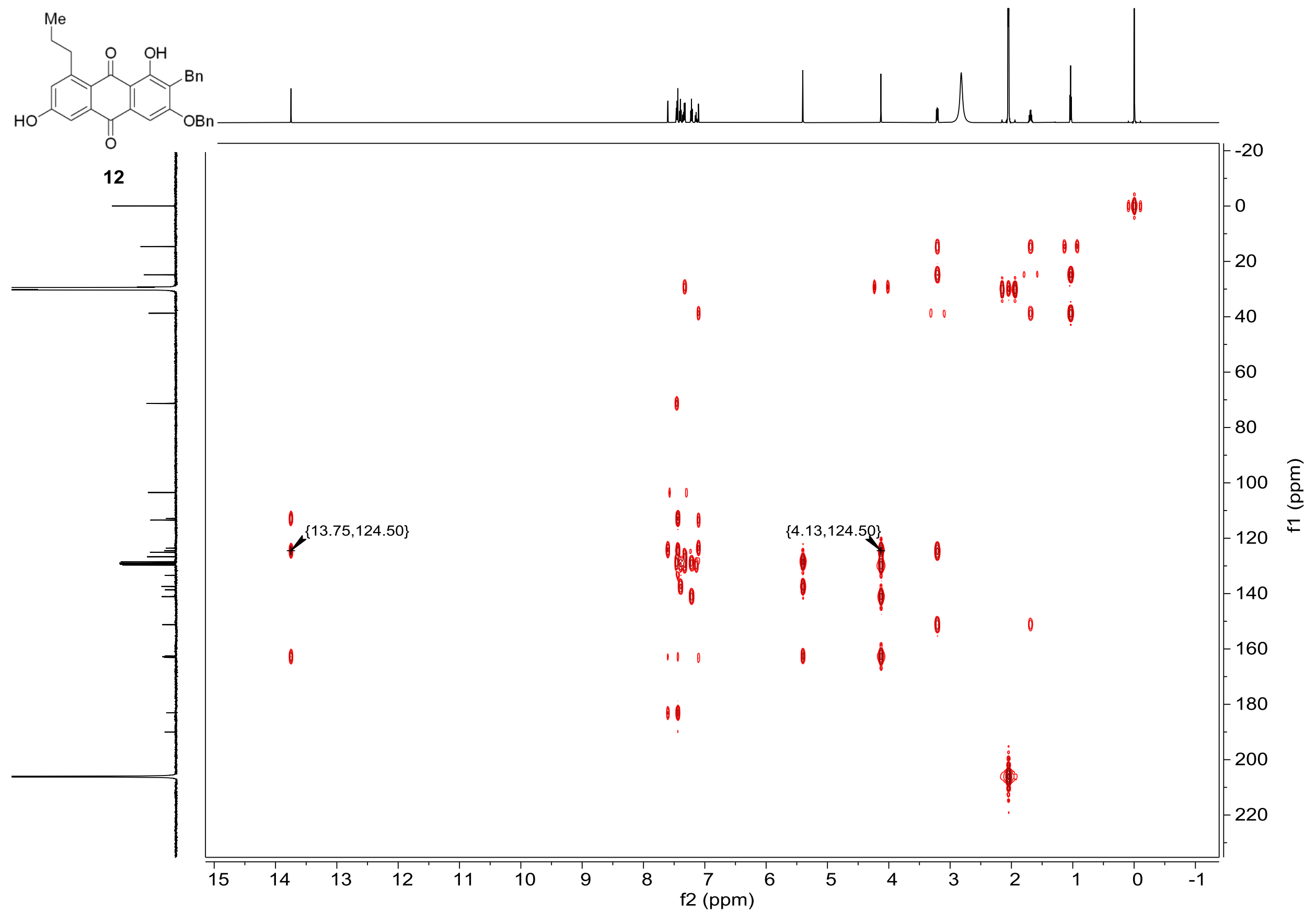

Figure S26. HMBC spectrum of $\mathbf{1 2}$ in acetone- $d_{6}$ 


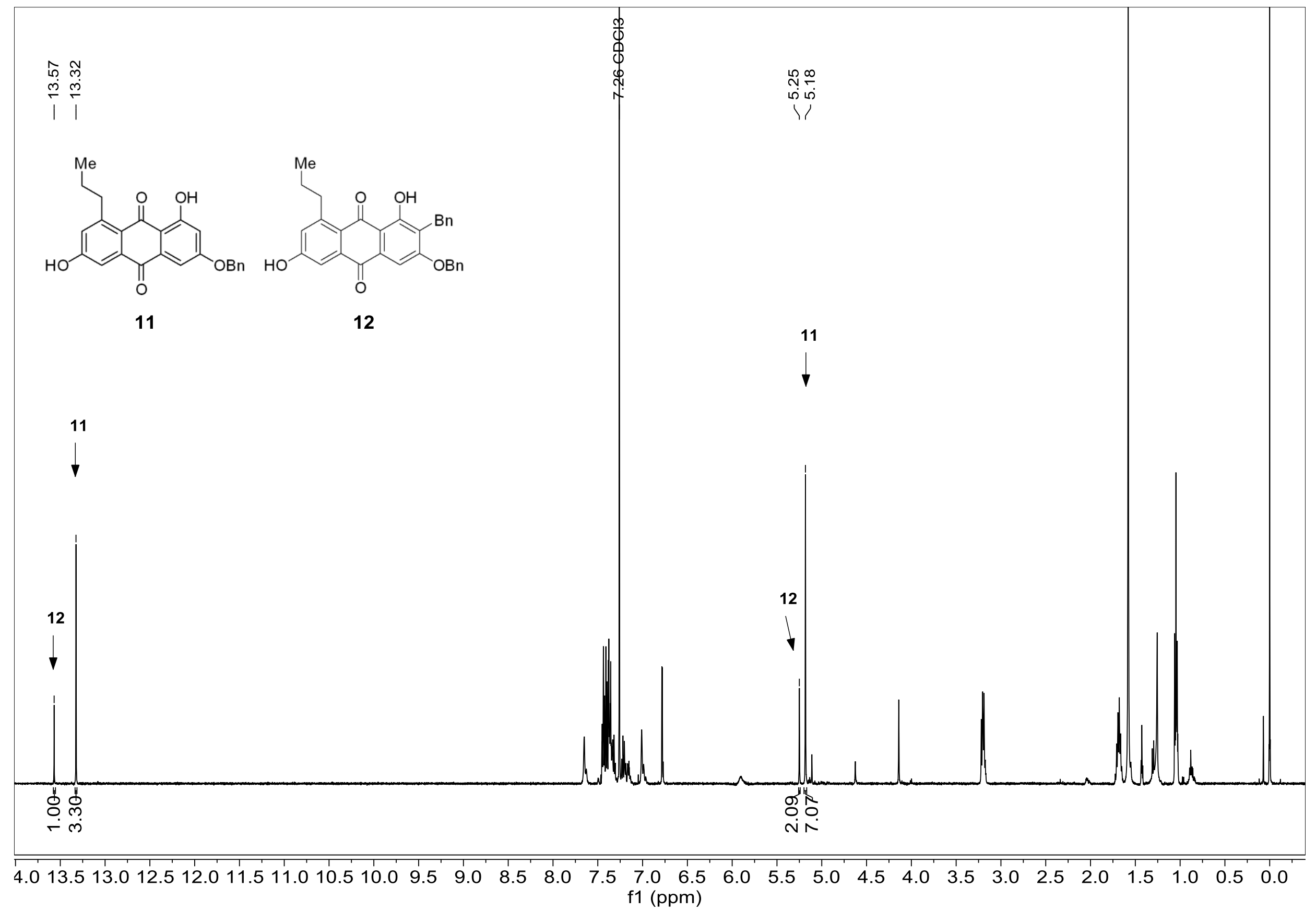

Figure S27. ${ }^{1} \mathrm{H}$ NMR spectrum of the mixture of 11 and $12\left(500 \mathrm{MHz}, \mathrm{CDCl}_{3}\right)$ 


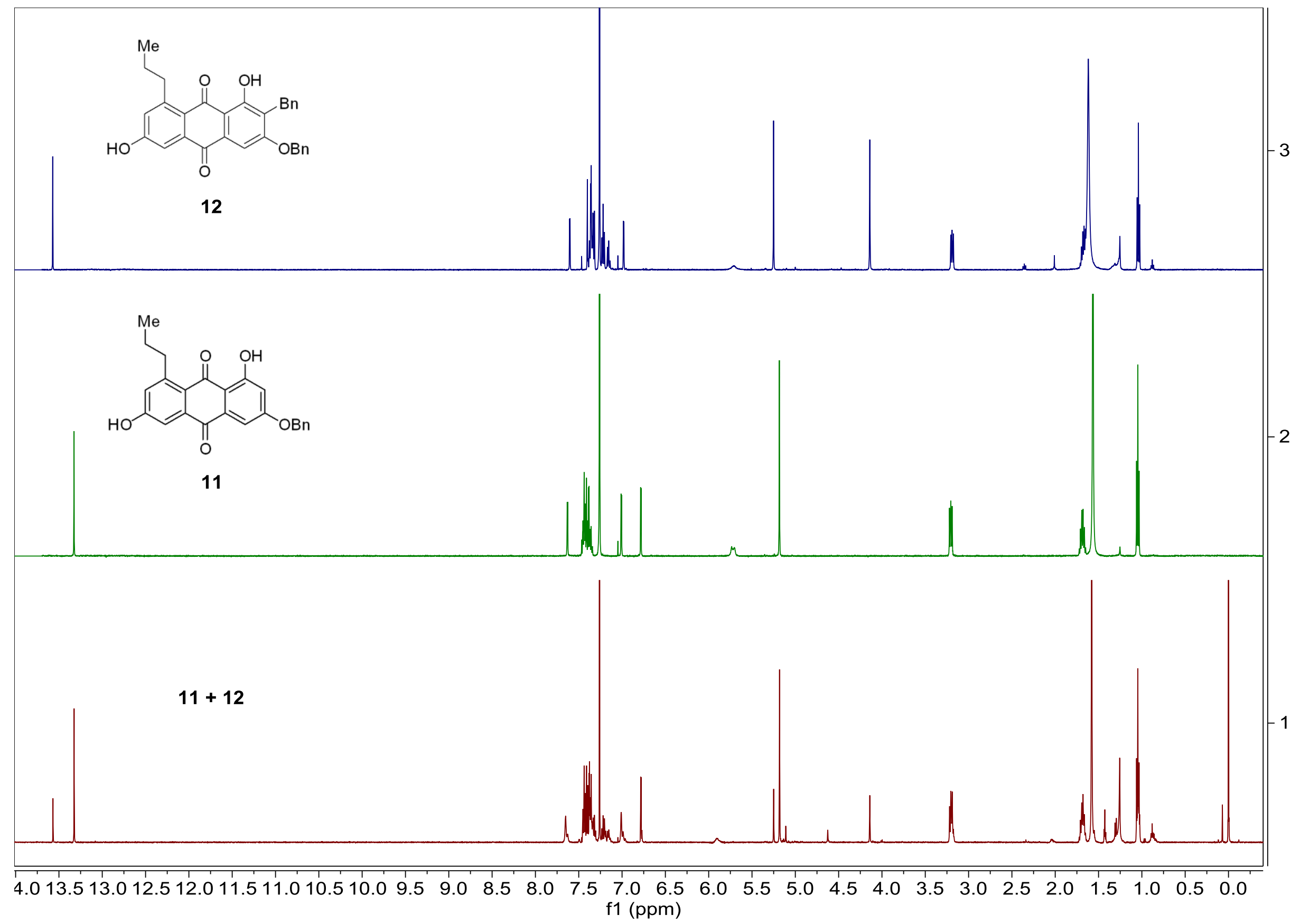

Figure S28. ${ }^{1} \mathrm{H}$ NMR spectrum of $\mathbf{1 1}, 12$ and their mixture (500 $\mathrm{MHz}, \mathrm{CDCl}_{3}$ ) 


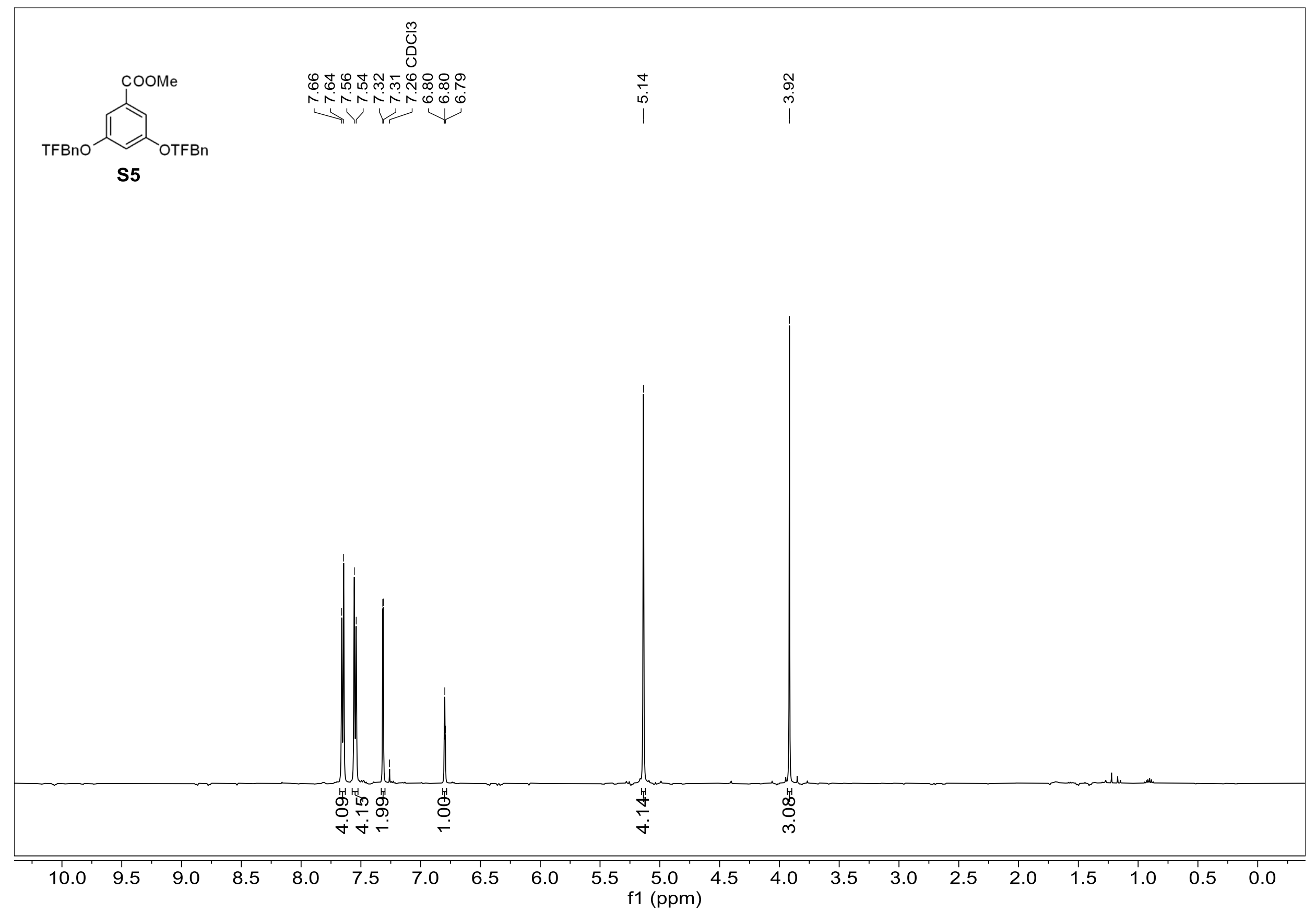

Figure S29. ${ }^{1} \mathrm{H}$ NMR spectrum of $\mathbf{S 5}\left(500 \mathrm{MHz}, \mathrm{CDCl}_{3}\right)$ 


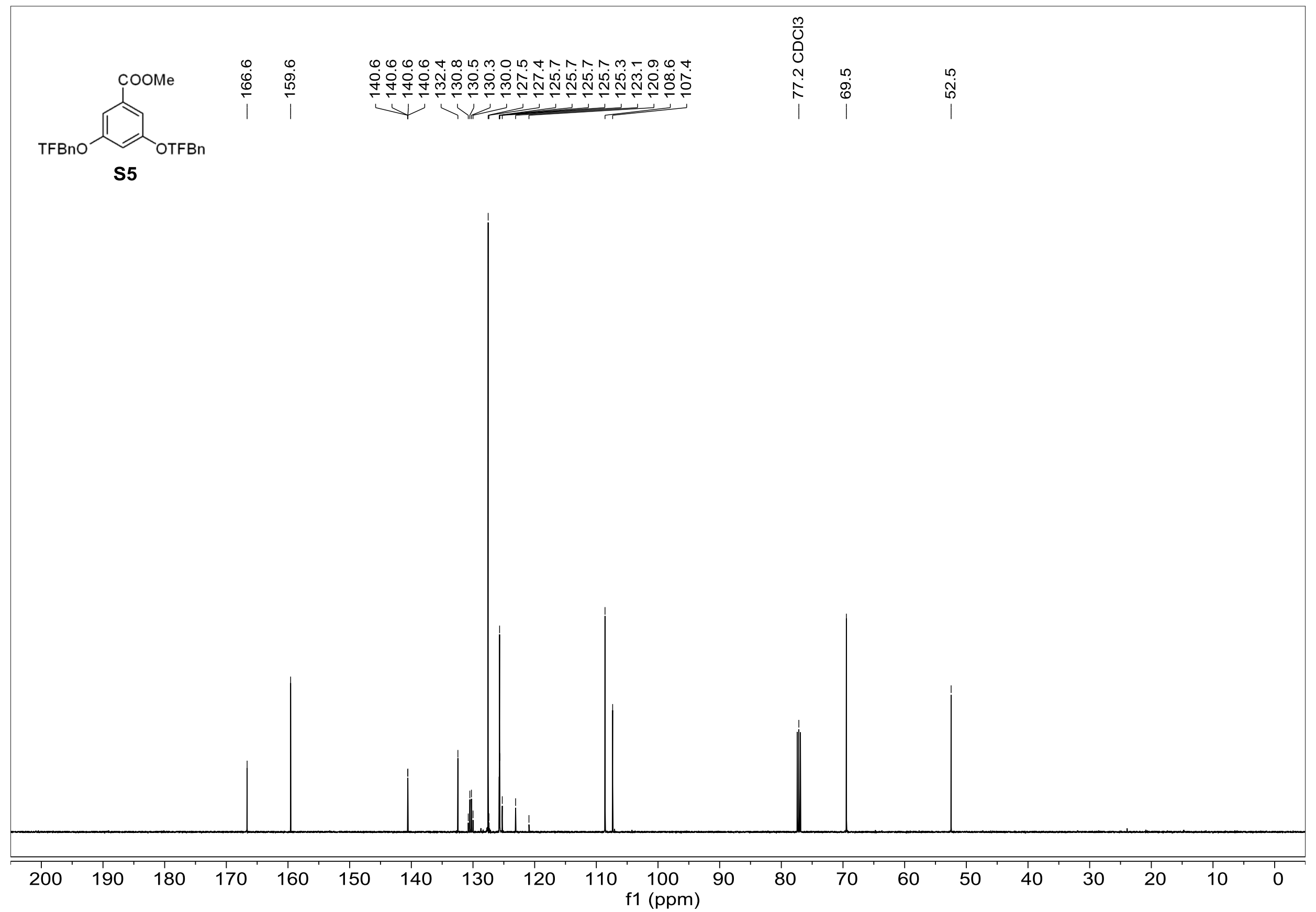

Figure S30. ${ }^{13} \mathrm{C}$ NMR spectrum of $\mathbf{S 5}\left(126 \mathrm{MHz}, \mathrm{CDCl}_{3}\right)$ 


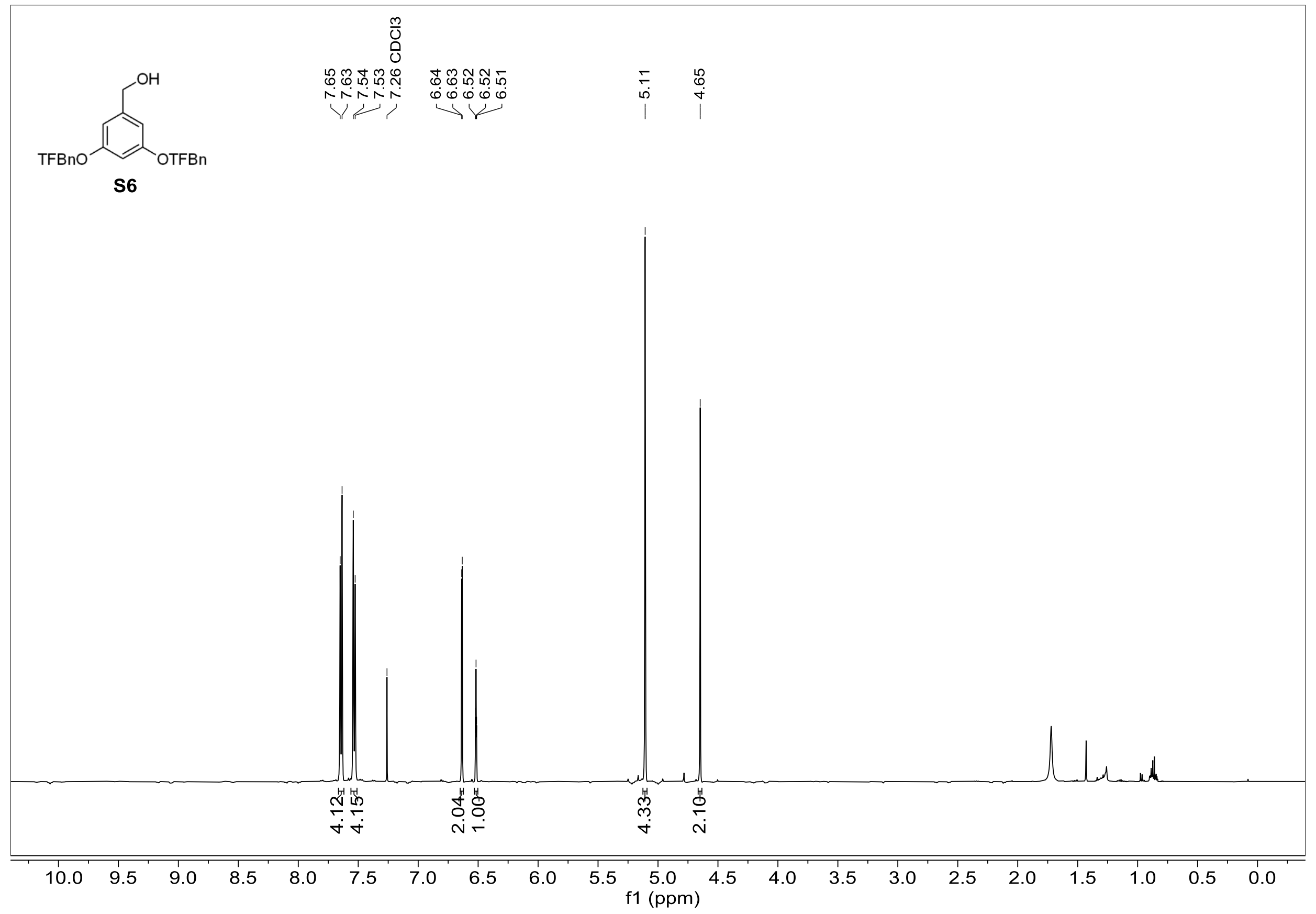

Figure S31. ${ }^{1} \mathrm{H}$ NMR spectrum of $\mathbf{S 6}\left(500 \mathrm{MHz}, \mathrm{CDCl}_{3}\right)$ 


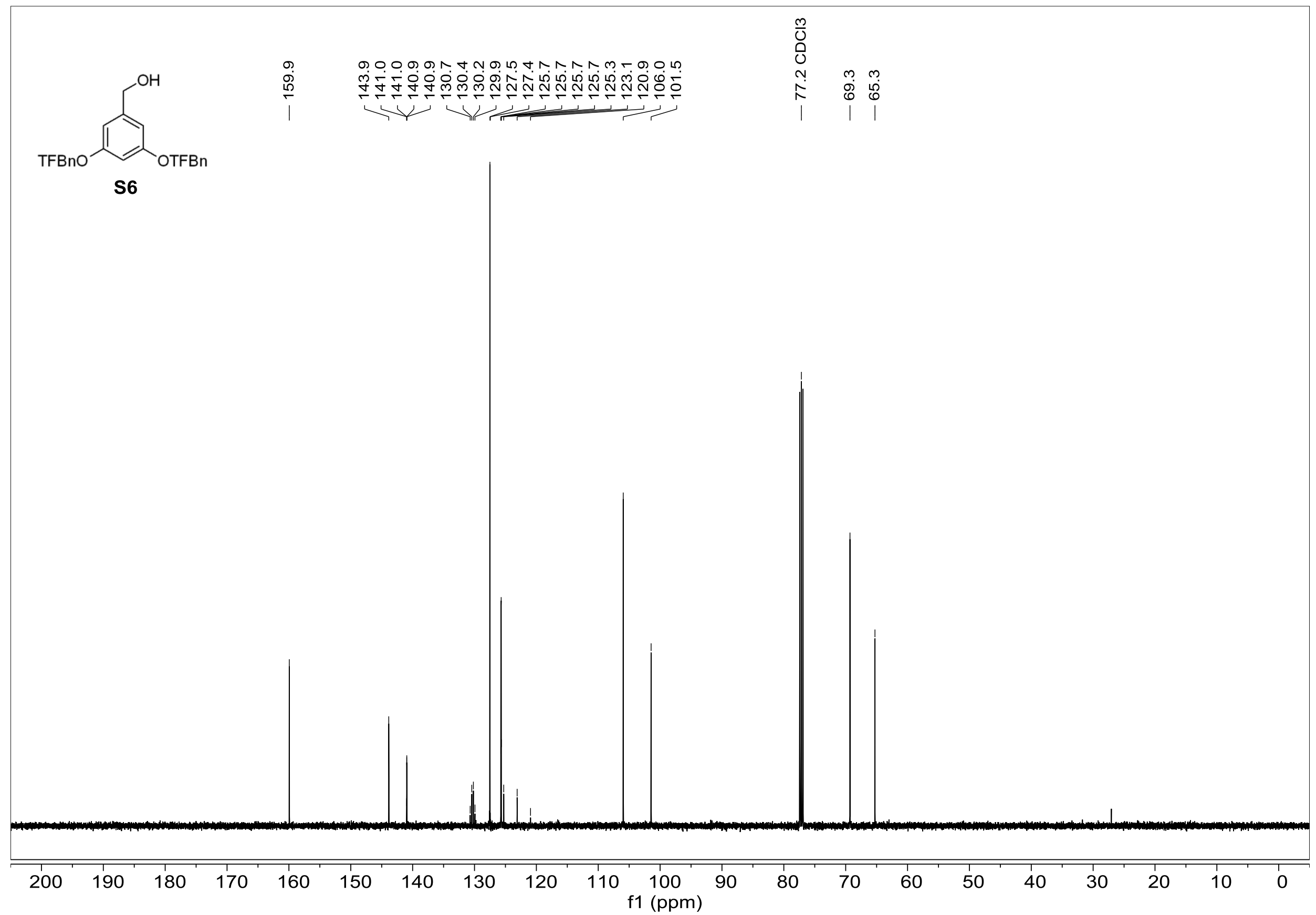

Figure S32. ${ }^{13} \mathrm{C}$ NMR spectrum of $\mathbf{S 6}\left(126 \mathrm{MHz}, \mathrm{CDCl}_{3}\right)$ 


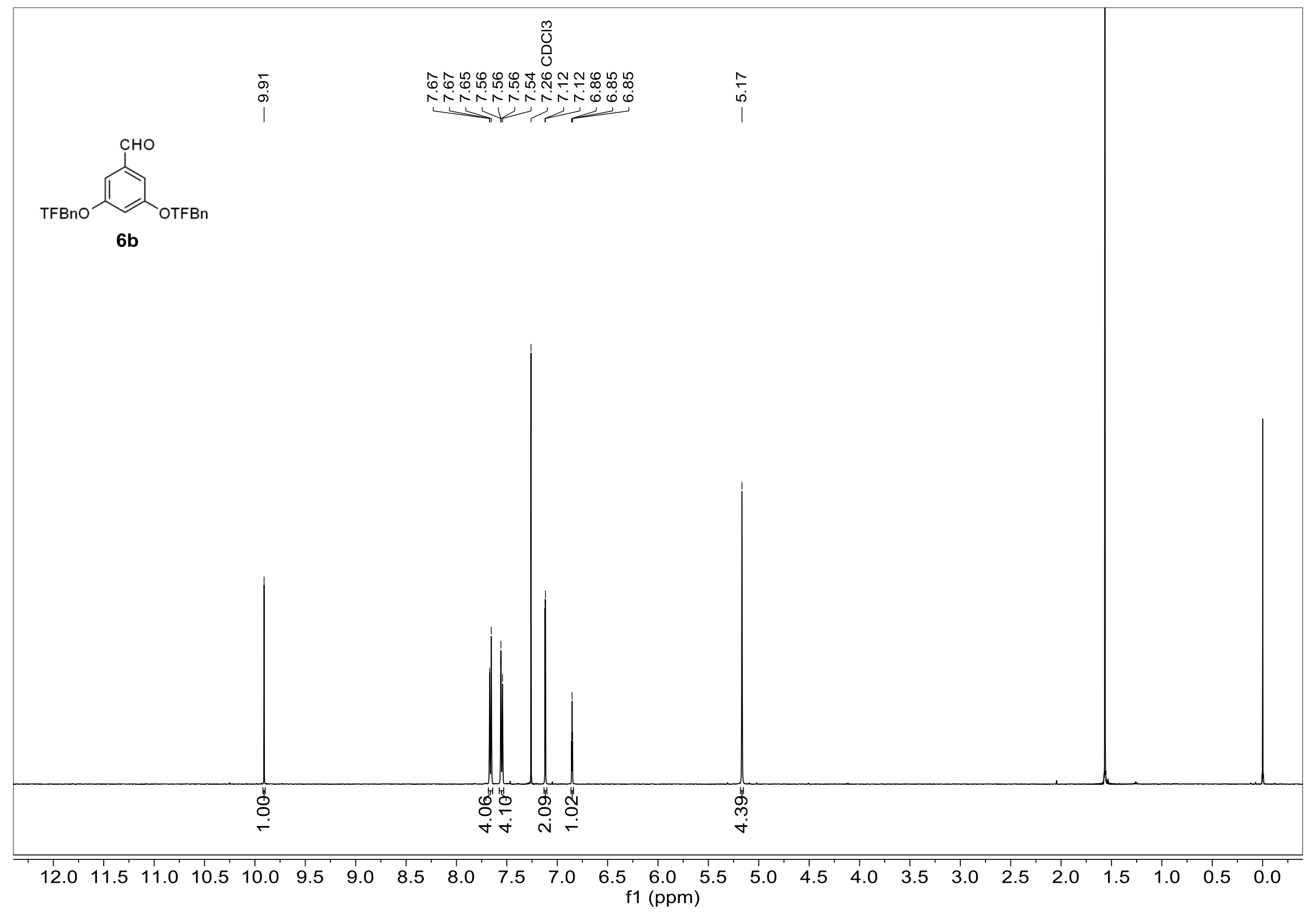

Figure S33. ${ }^{1} \mathrm{H}$ NMR spectrum of $\mathbf{6 b}\left(500 \mathrm{MHz}, \mathrm{CDCl}_{3}\right)$ 


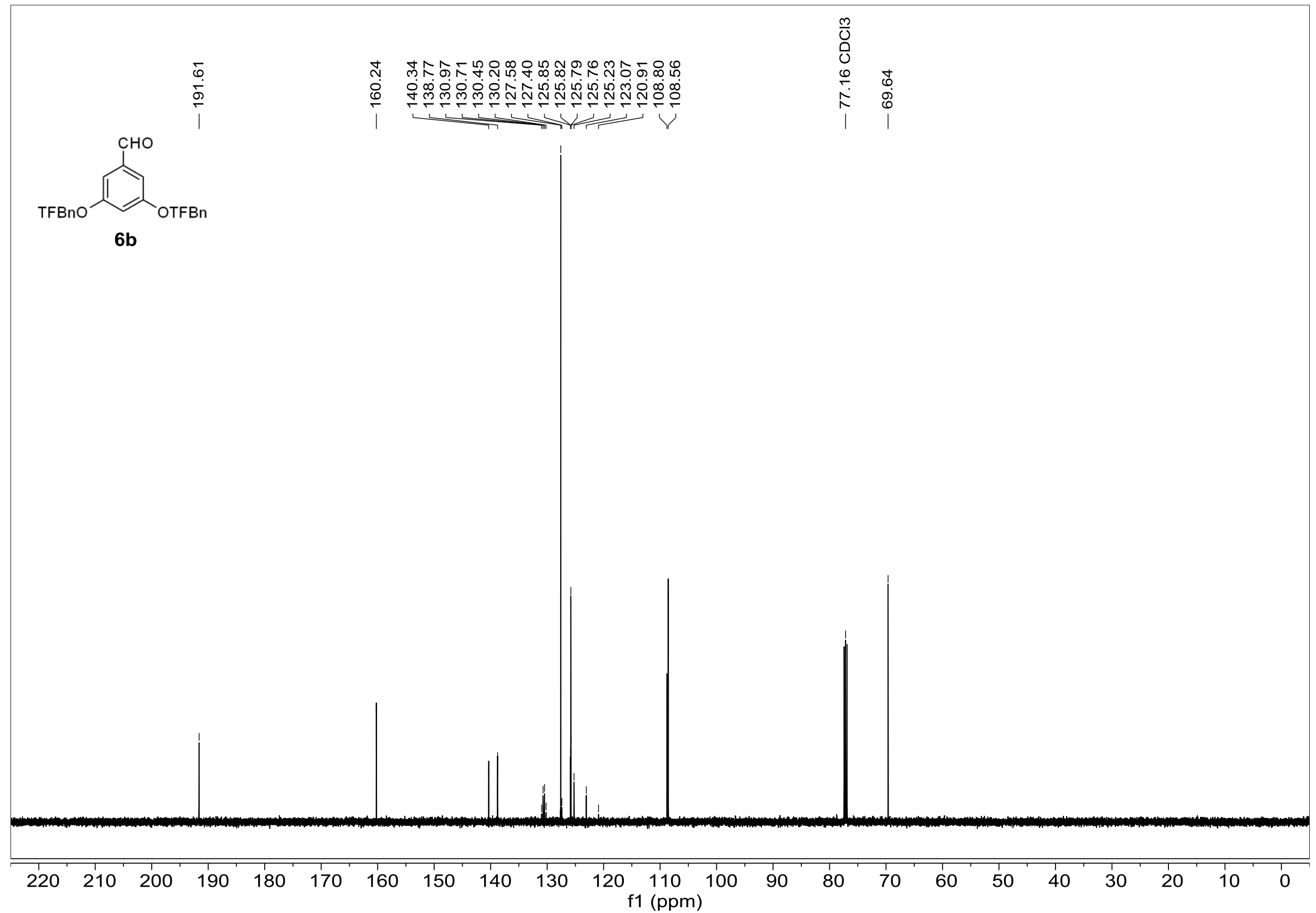

Figure S34. ${ }^{13} \mathrm{C}$ NMR spectrum of $\mathbf{6 b}\left(126 \mathrm{MHz}, \mathrm{CDCl}_{3}\right)$ 


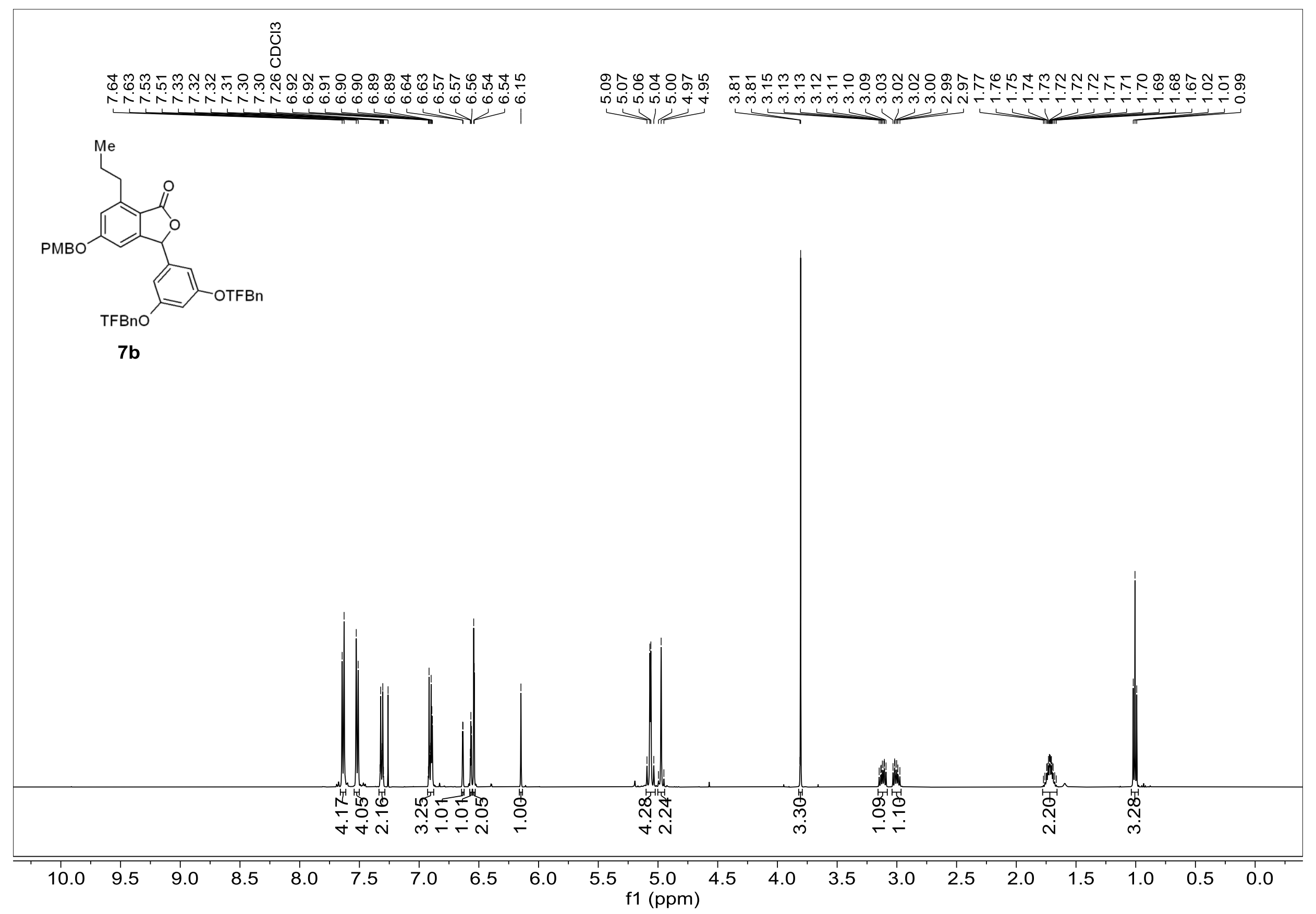

Figure S35. ${ }^{1} \mathrm{H}$ NMR spectrum of $\mathbf{7 b}\left(500 \mathrm{MHz}, \mathrm{CDCl}_{3}\right)$ 


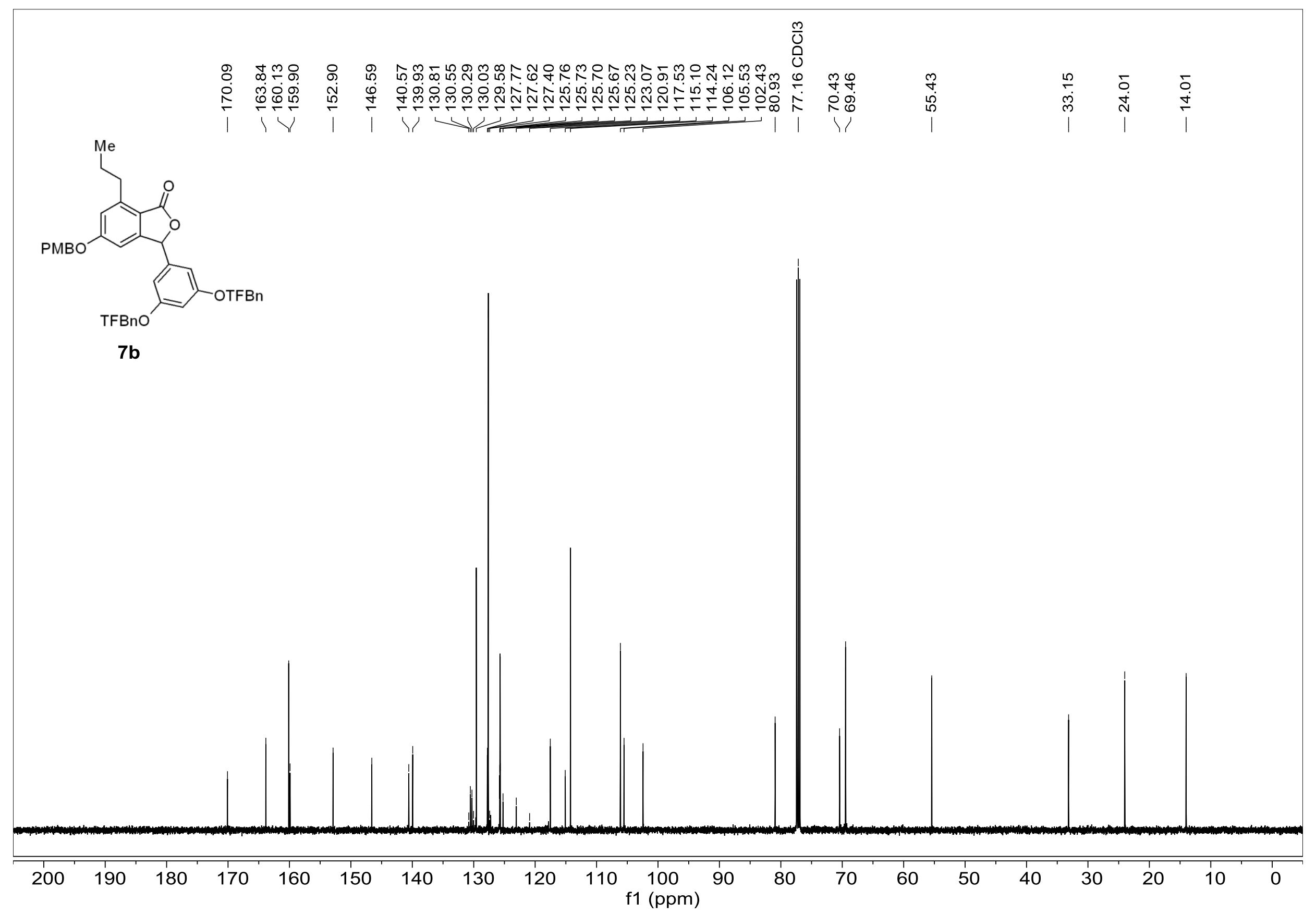

Figure S36. ${ }^{13} \mathrm{C}$ NMR spectrum of $\mathbf{7 b}\left(126 \mathrm{MHz}, \mathrm{CDCl}_{3}\right)$ 


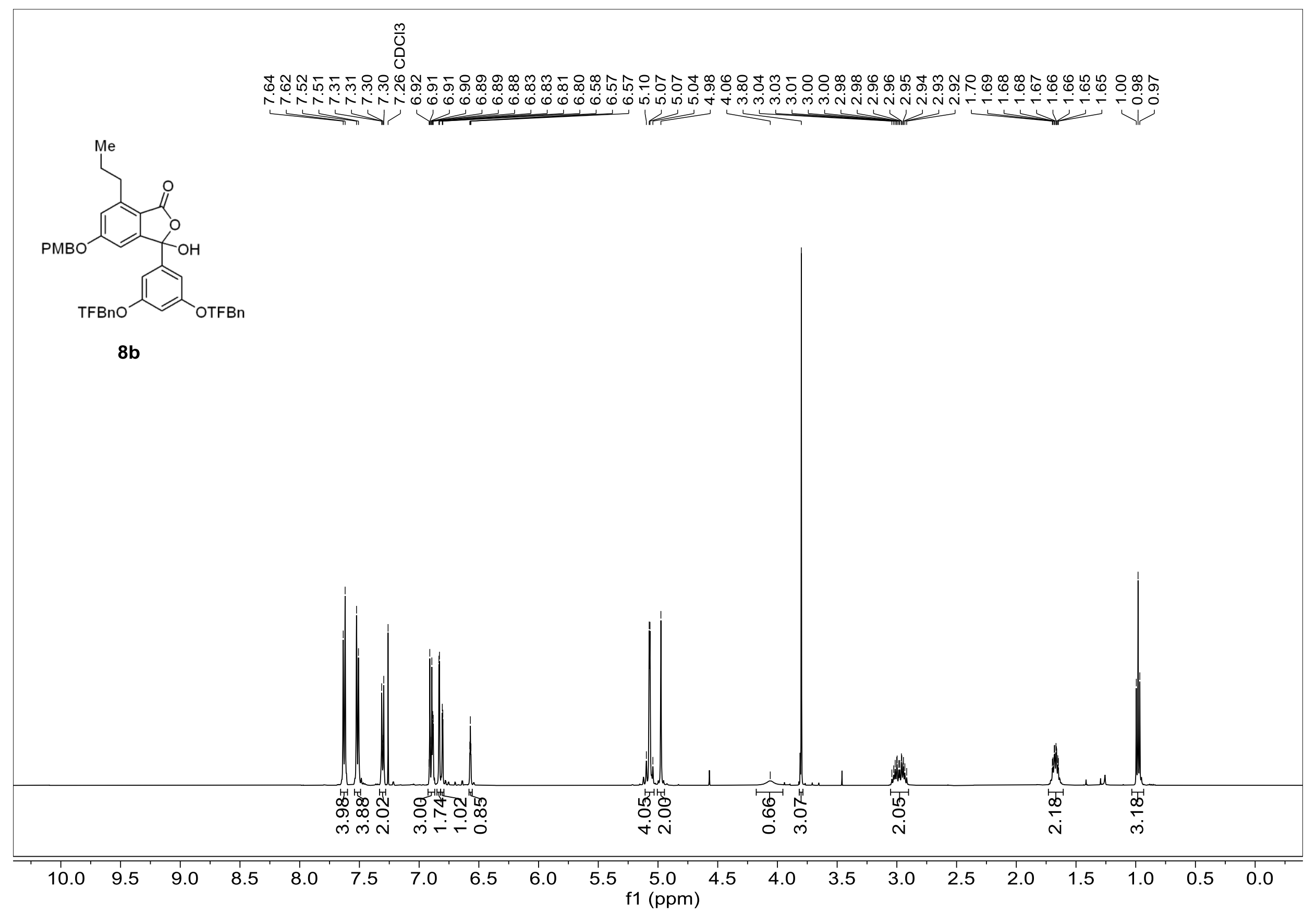

Figure S37. ${ }^{1} \mathrm{H}$ NMR spectrum of $\mathbf{8 b}\left(500 \mathrm{MHz}, \mathrm{CDCl}_{3}\right)$ 


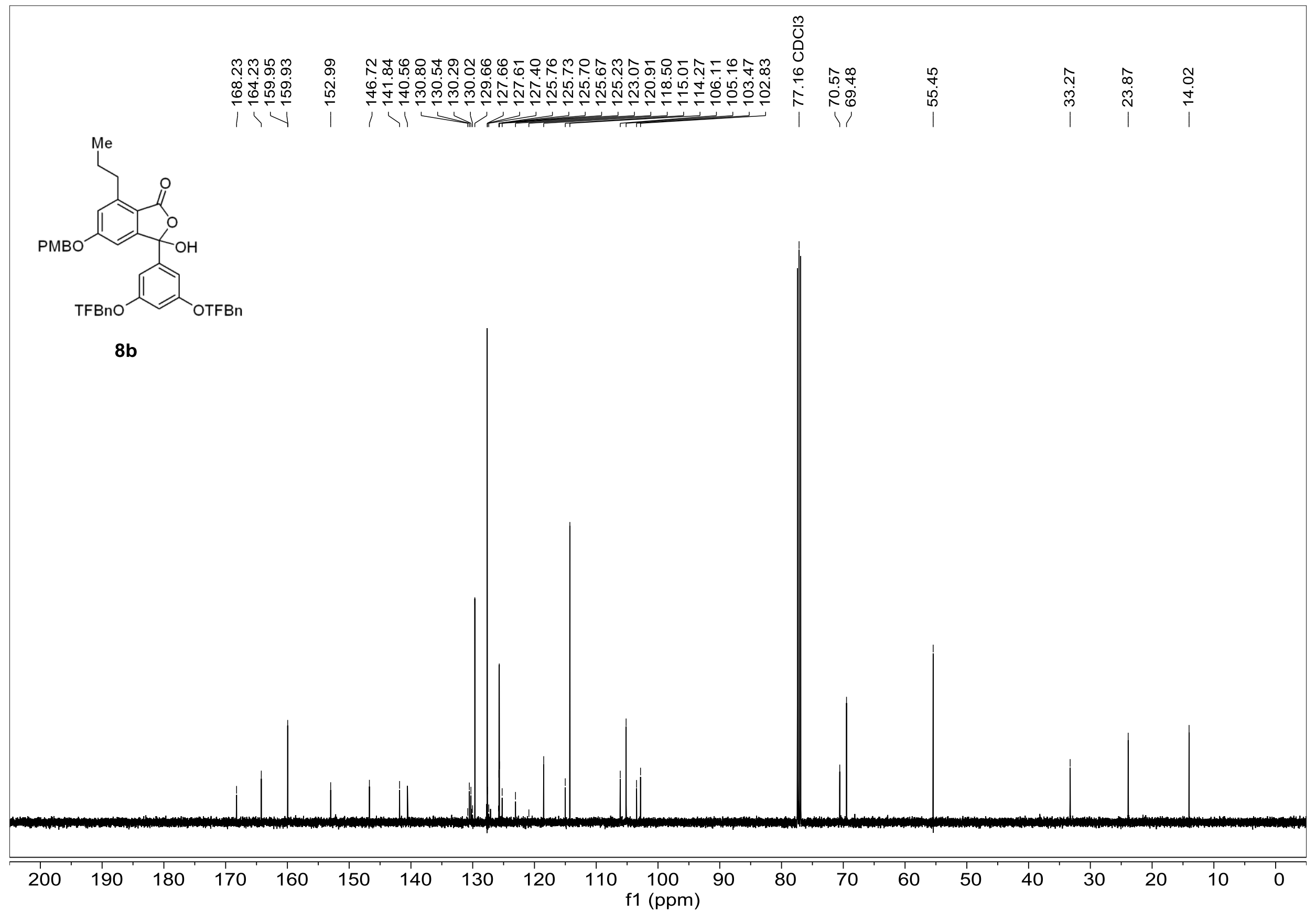

Figure S38. ${ }^{13} \mathrm{C}$ NMR spectrum of $\mathbf{8 b}\left(126 \mathrm{MHz}, \mathrm{CDCl}_{3}\right)$ 


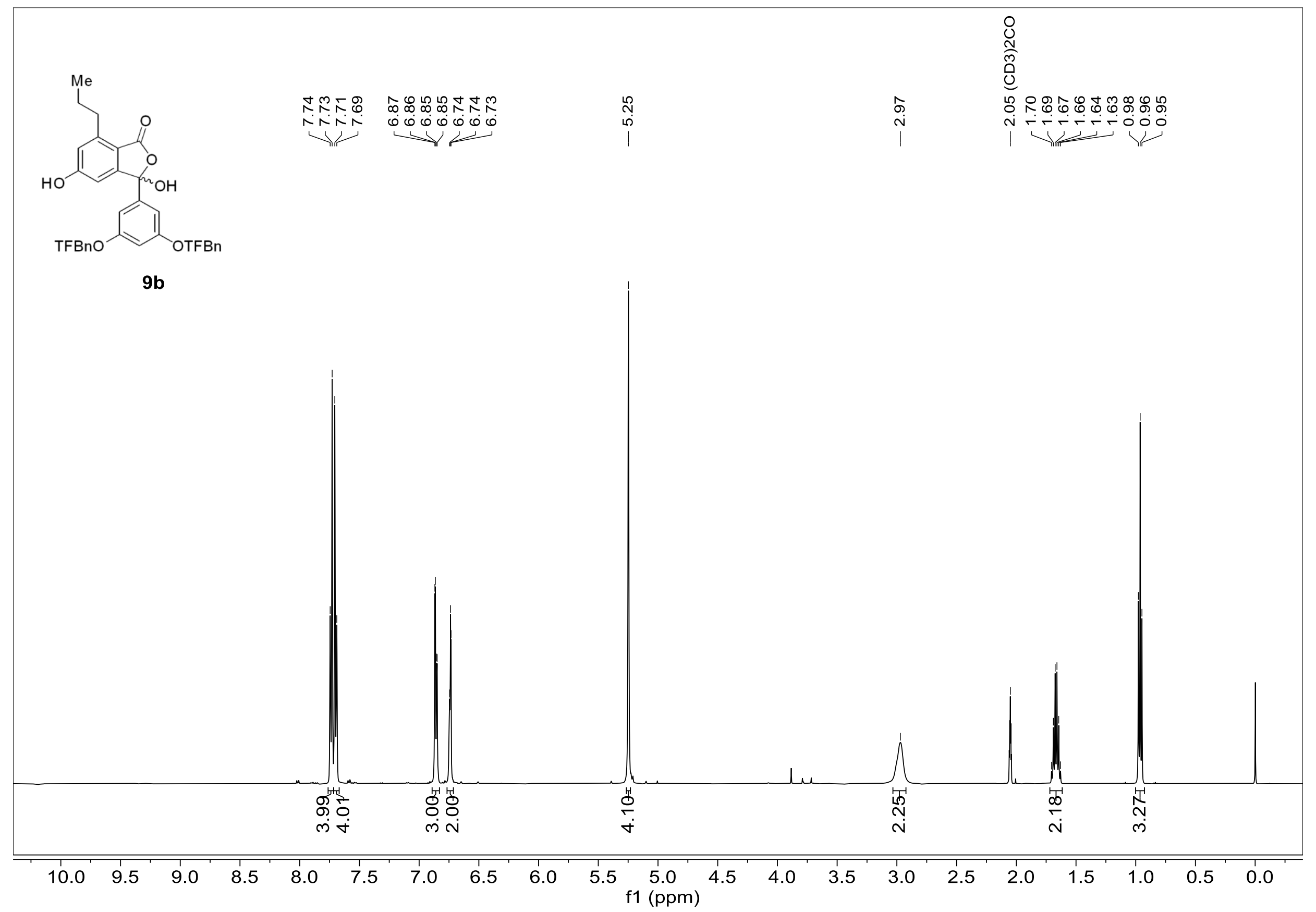

Figure S39. ${ }^{1} \mathrm{H}$ NMR spectrum of $\mathbf{9 b}\left(500 \mathrm{MHz}\right.$, acetone- $d_{6}$ ) 


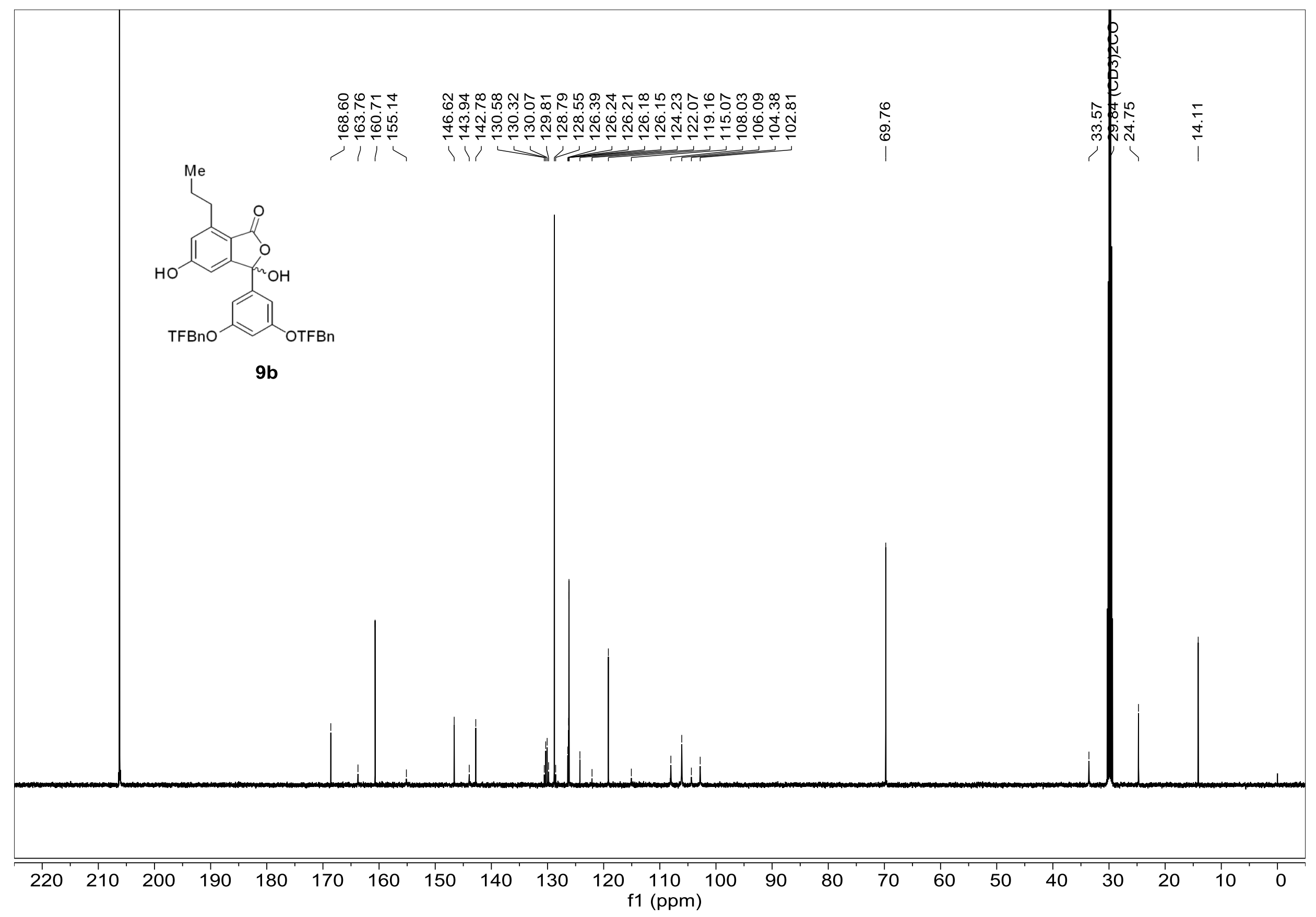

Figure S40. ${ }^{13} \mathrm{C}$ NMR spectrum of $9 \mathbf{b}\left(126 \mathrm{MHz}\right.$, acetone- $\left.d_{6}\right)$ 


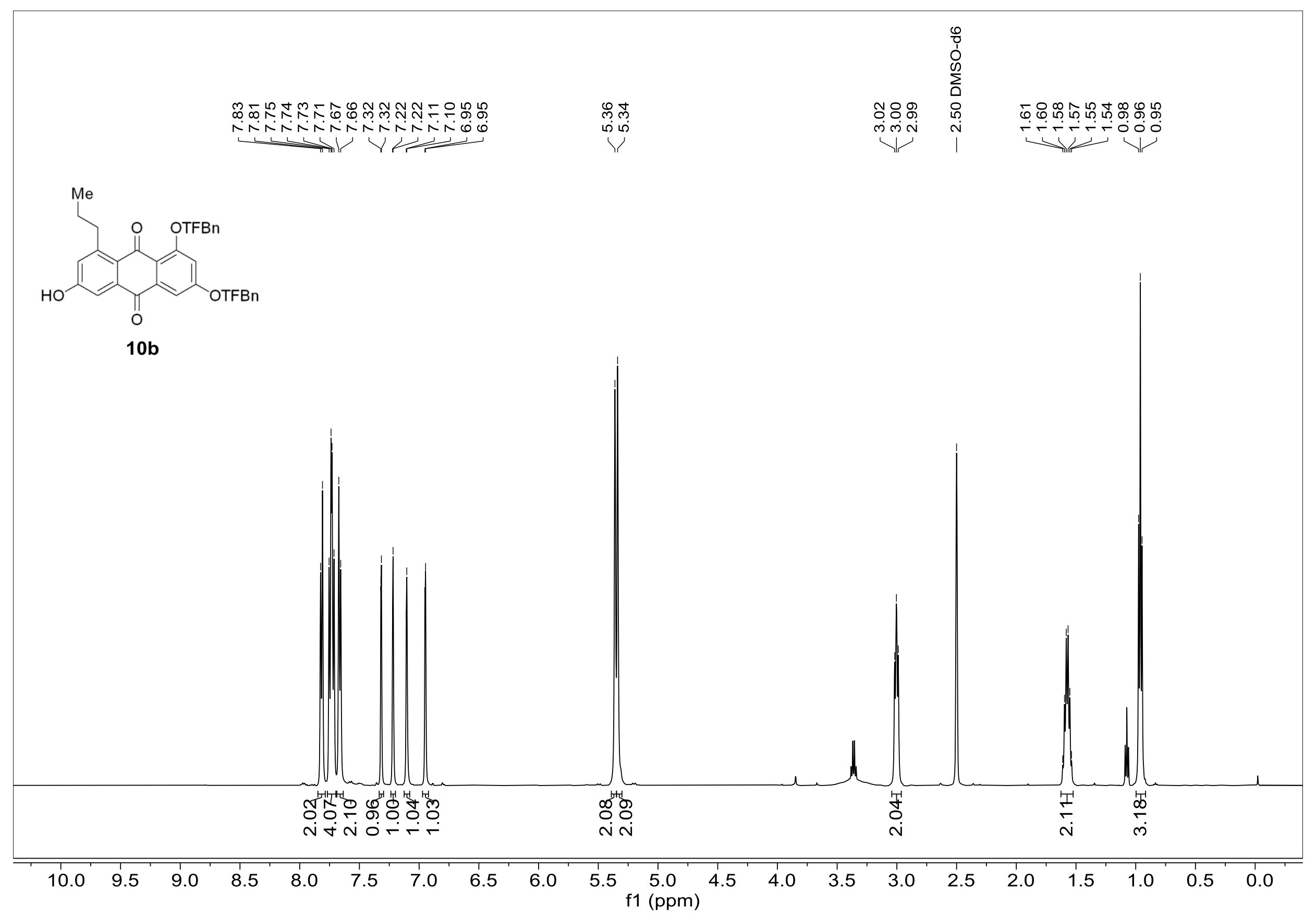

Figure S41. ${ }^{1} \mathrm{H}$ NMR spectrum of $\mathbf{1 0 b}\left(500 \mathrm{MHz}, \mathrm{DMSO}-d_{6}\right)$ 


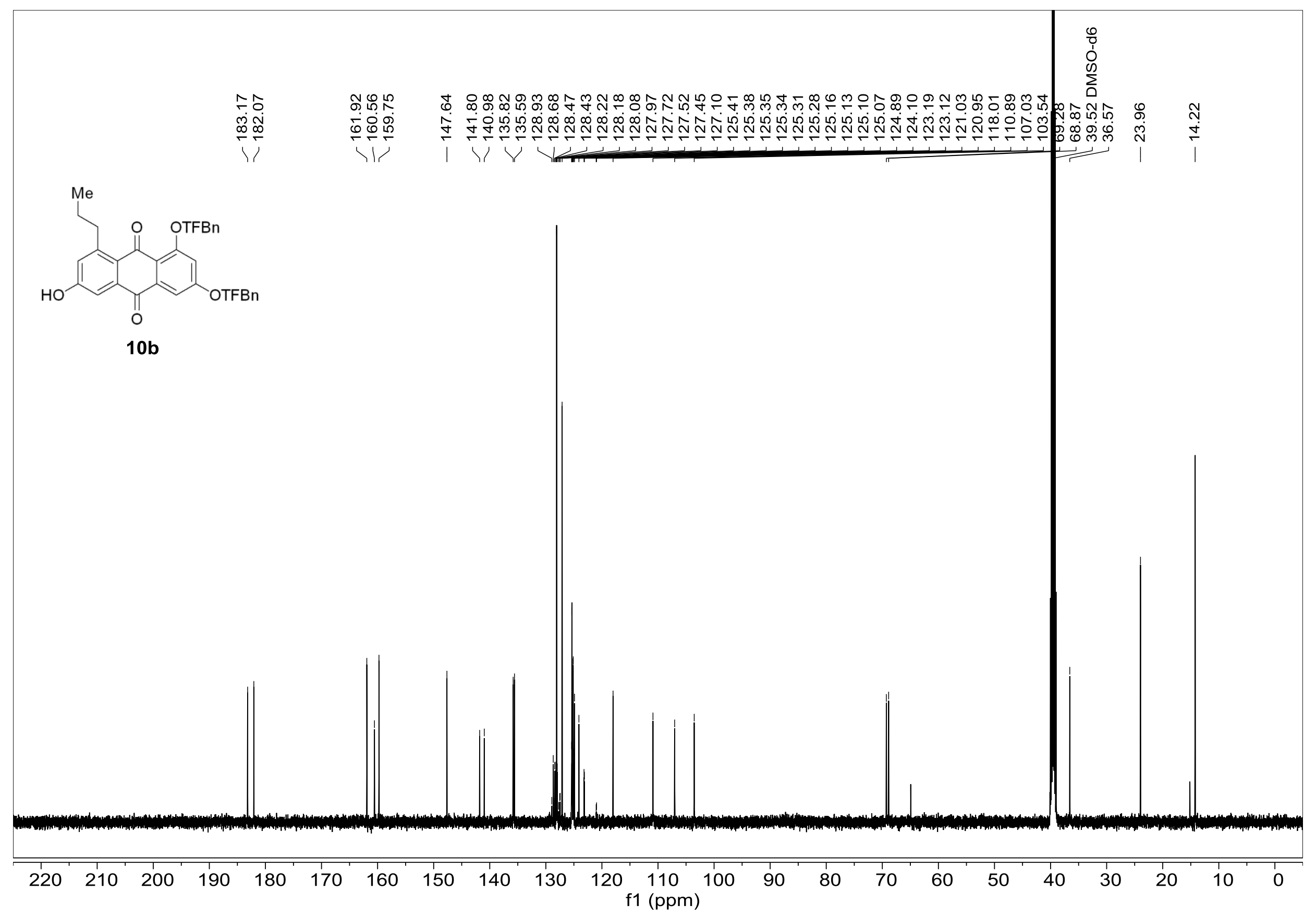

Figure S42. ${ }^{13} \mathrm{C}$ NMR spectrum of $\mathbf{1 0 b}\left(126 \mathrm{MHz}, \mathrm{DMSO}-d_{6}\right)$ 


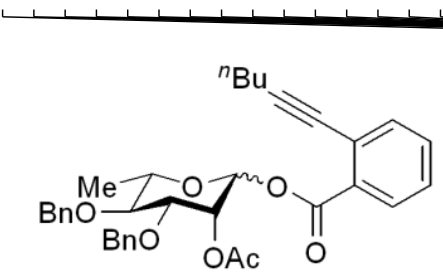

16

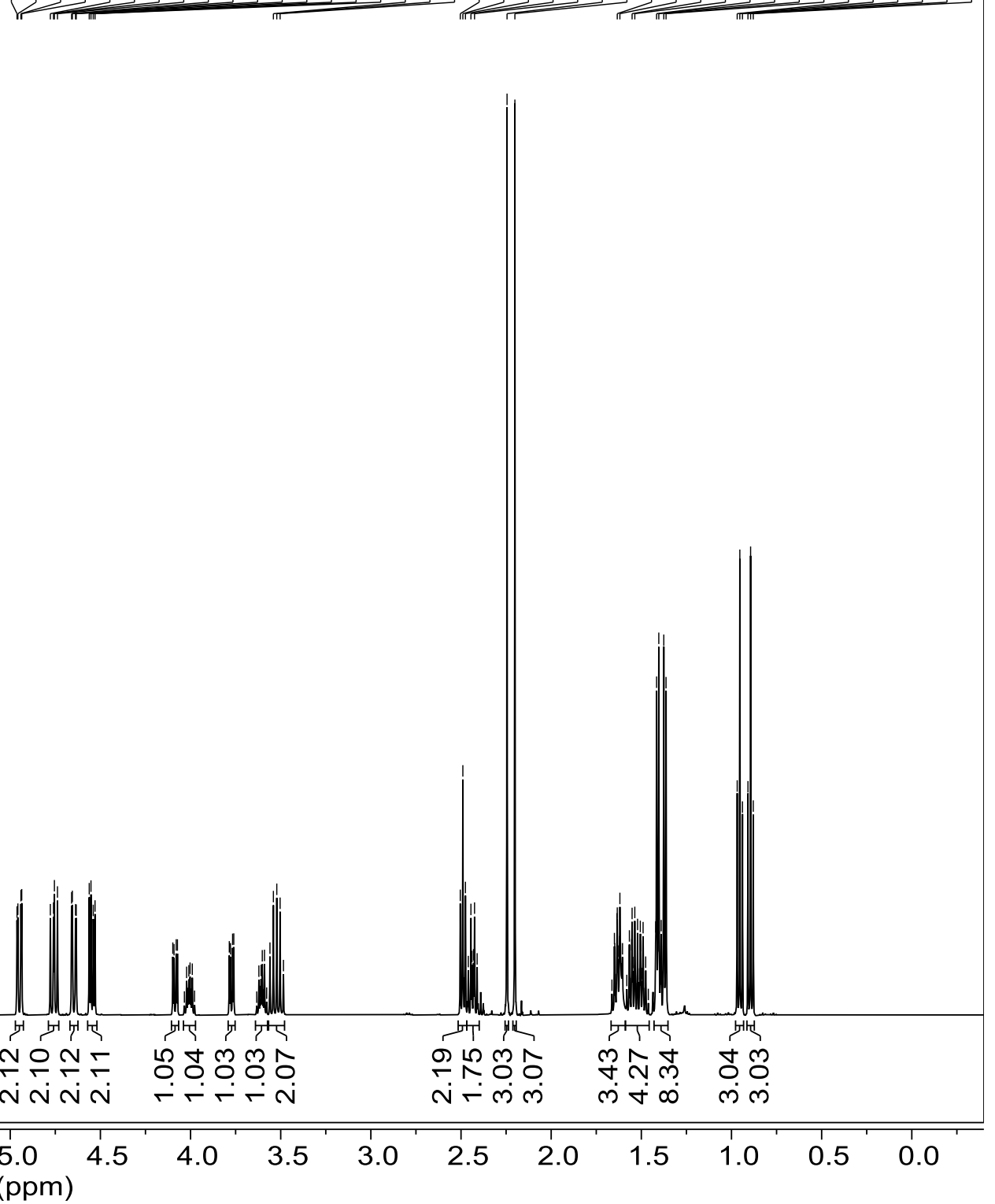

Figure S43. ${ }^{1} \mathrm{H}$ NMR spectrum of $16\left(500 \mathrm{MHz}, \mathrm{CDCl}_{3}\right)$ 


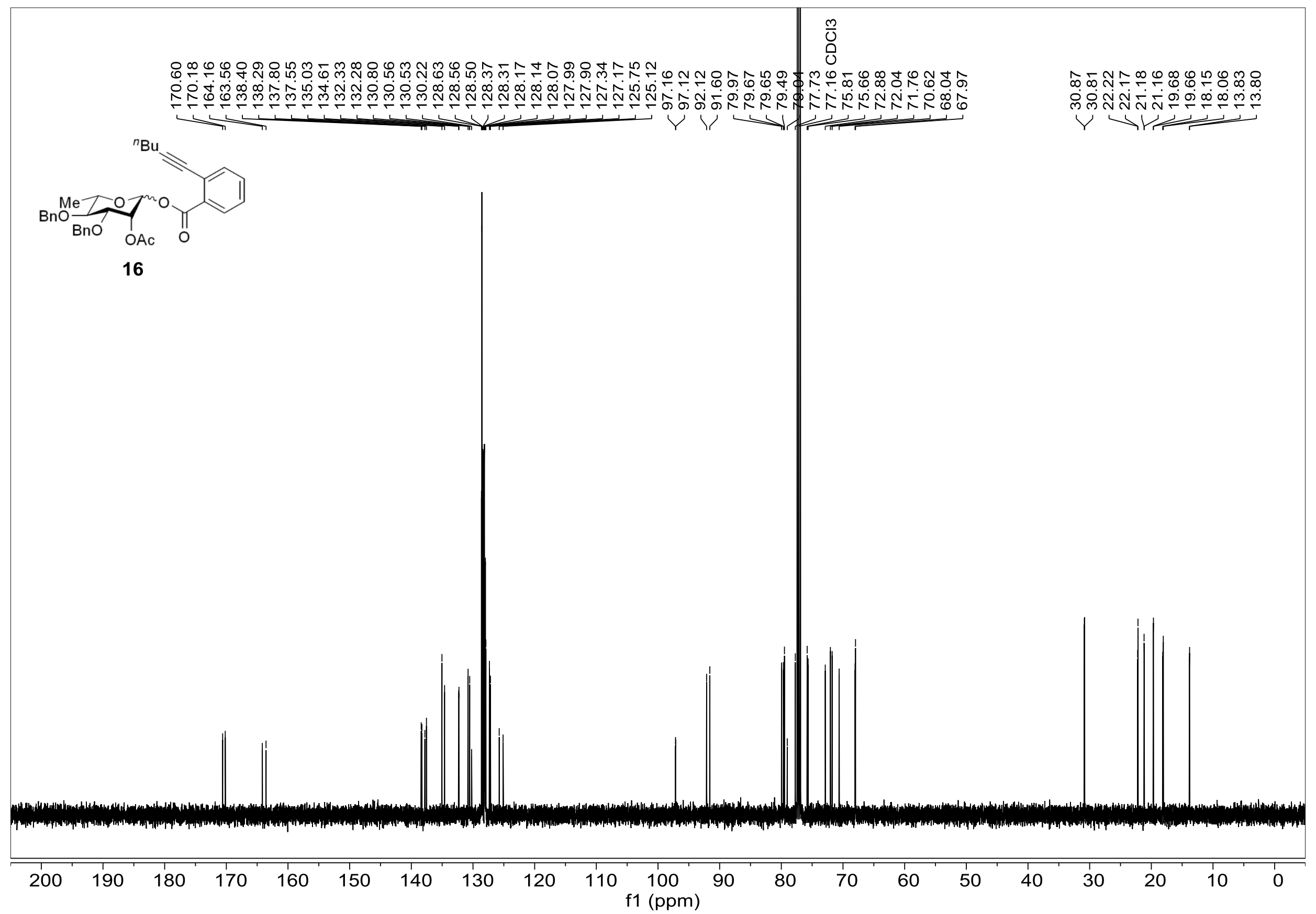

Figure S44. ${ }^{13} \mathrm{C}$ NMR spectrum of $\mathbf{1 6}\left(126 \mathrm{MHz}, \mathrm{CDCl}_{3}\right)$ 


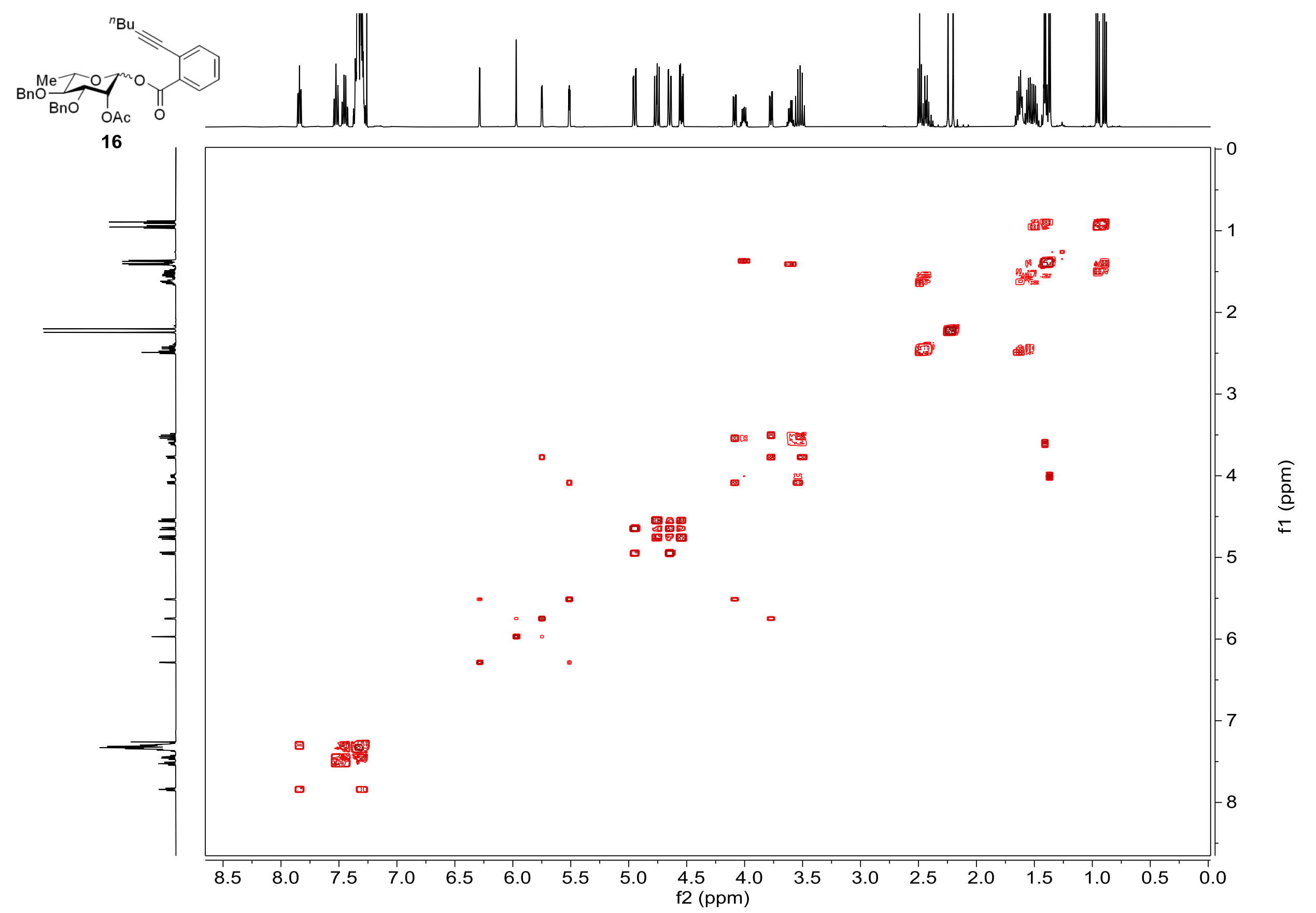

Figure S45. COSY spectrum of 16 in $\mathrm{CDCl}_{3}$ 


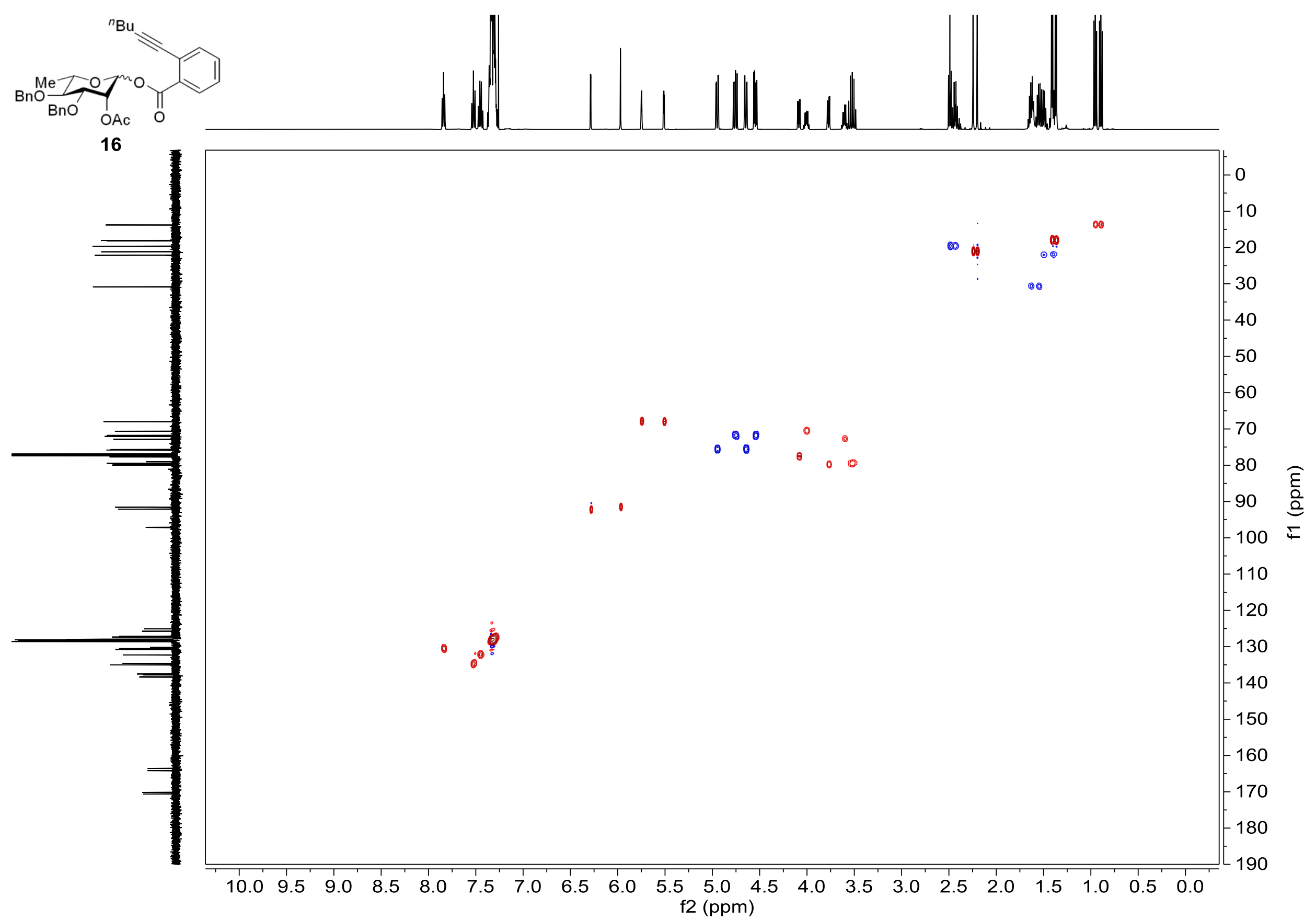

Figure S46. HSQC spectrum of 16 in $\mathrm{CDCl}_{3}$ 


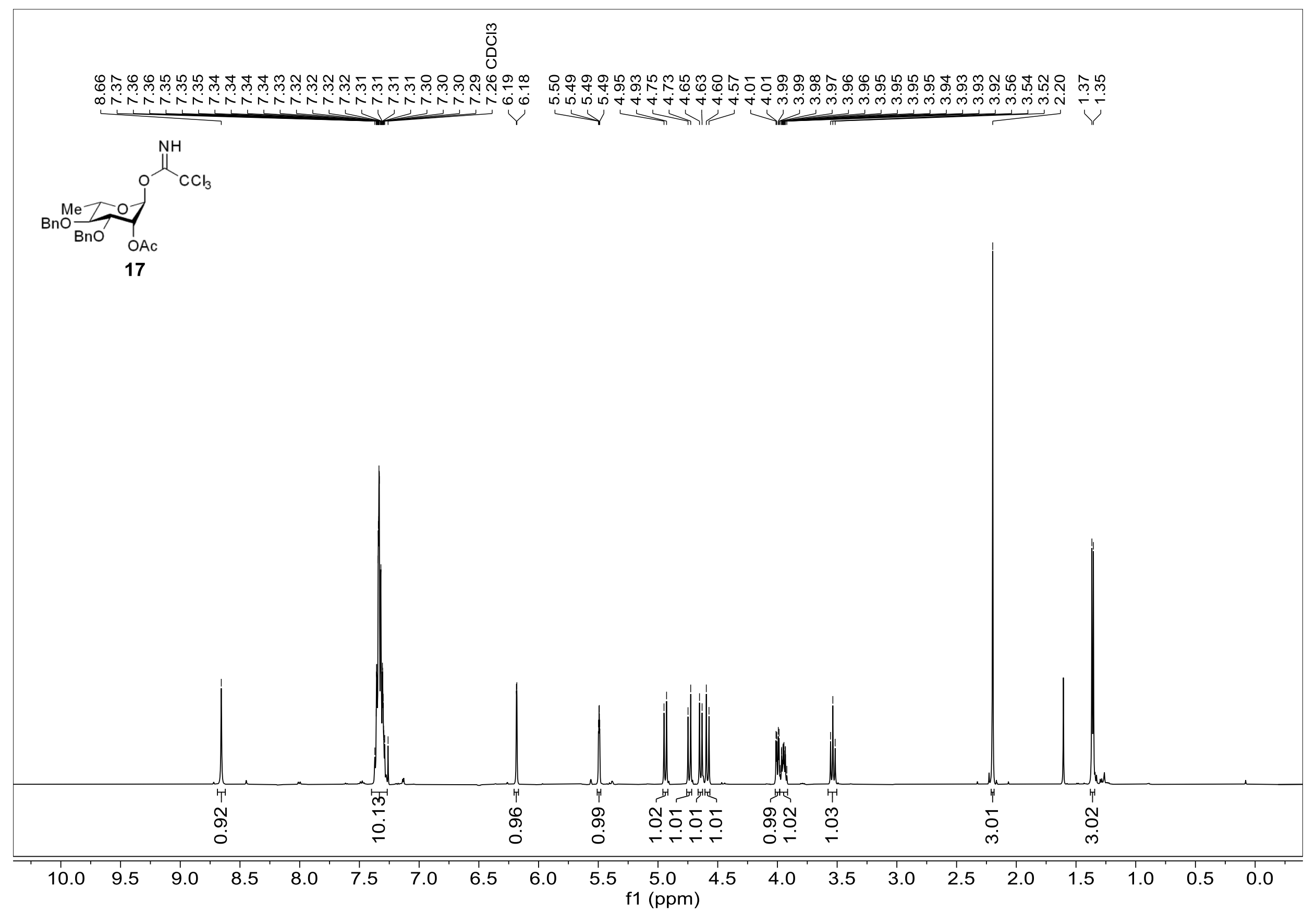

Figure S47. ${ }^{1} \mathrm{H}$ NMR spectrum of $17\left(500 \mathrm{MHz}, \mathrm{CDCl}_{3}\right)$ 


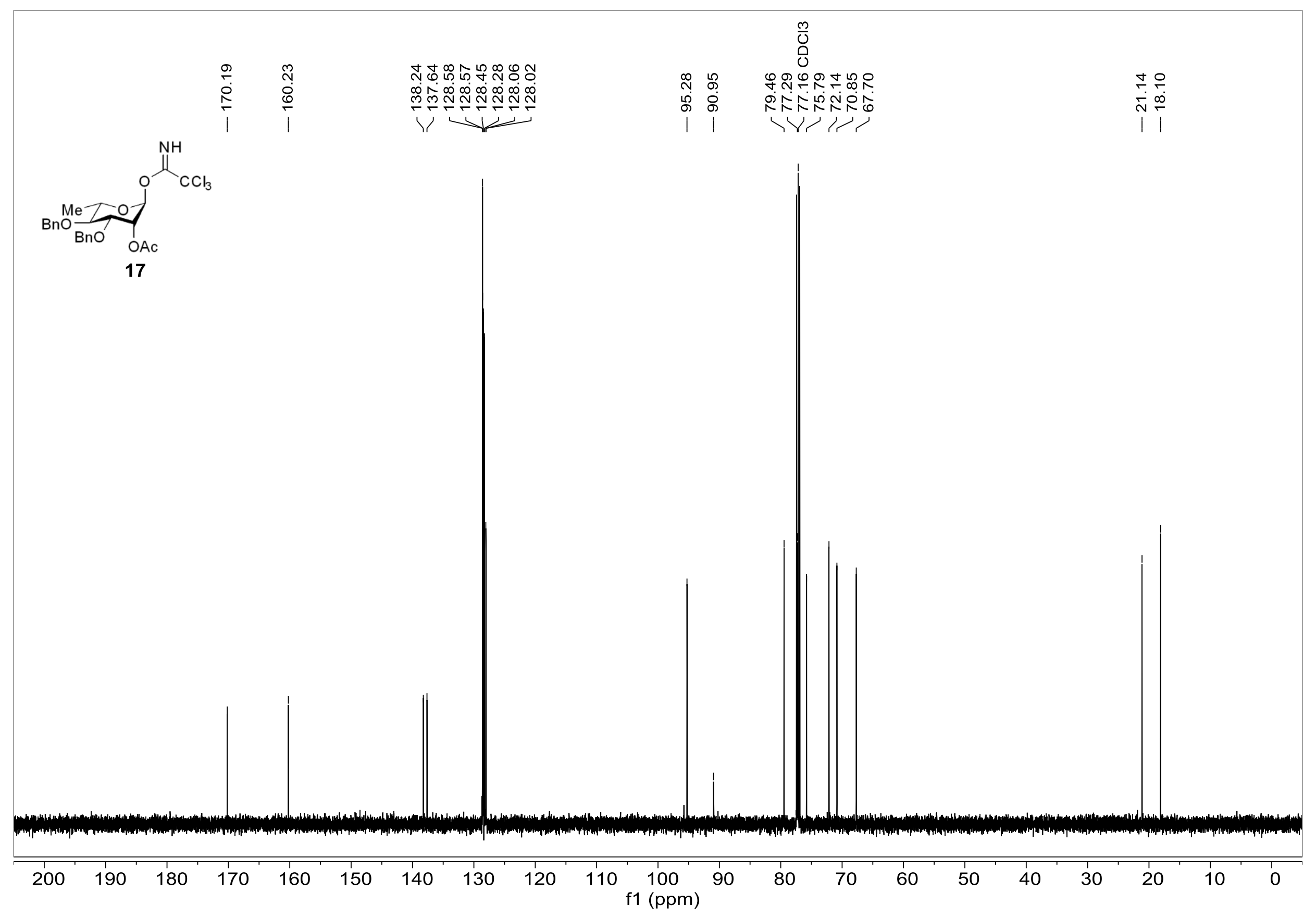

Figure S48. ${ }^{13} \mathrm{C}$ NMR spectrum of $17\left(126 \mathrm{MHz}, \mathrm{CDCl}_{3}\right)$ 


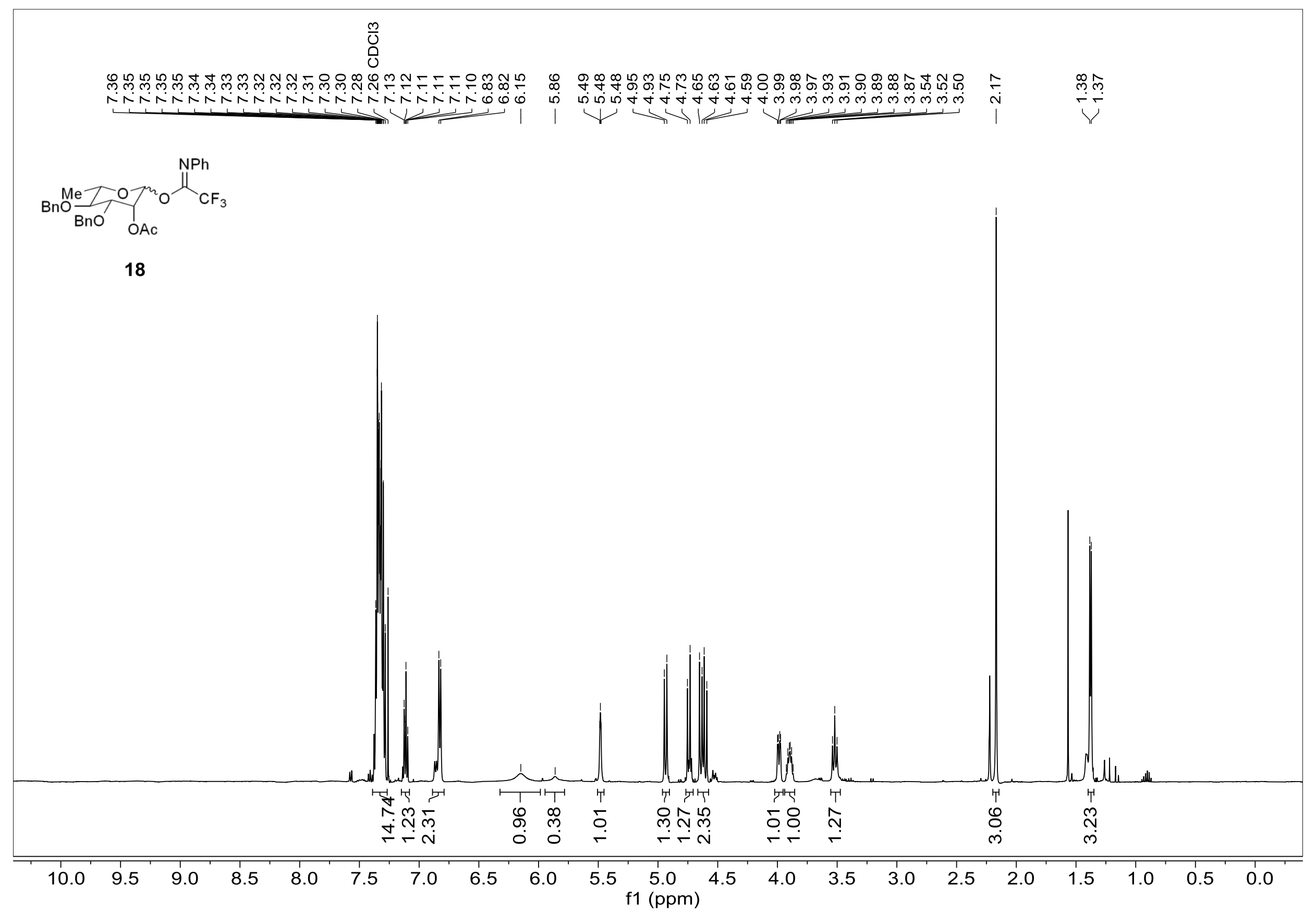

Figure S49. ${ }^{1} \mathrm{H}$ NMR spectrum of $18\left(500 \mathrm{MHz}, \mathrm{CDCl}_{3}\right)$ 


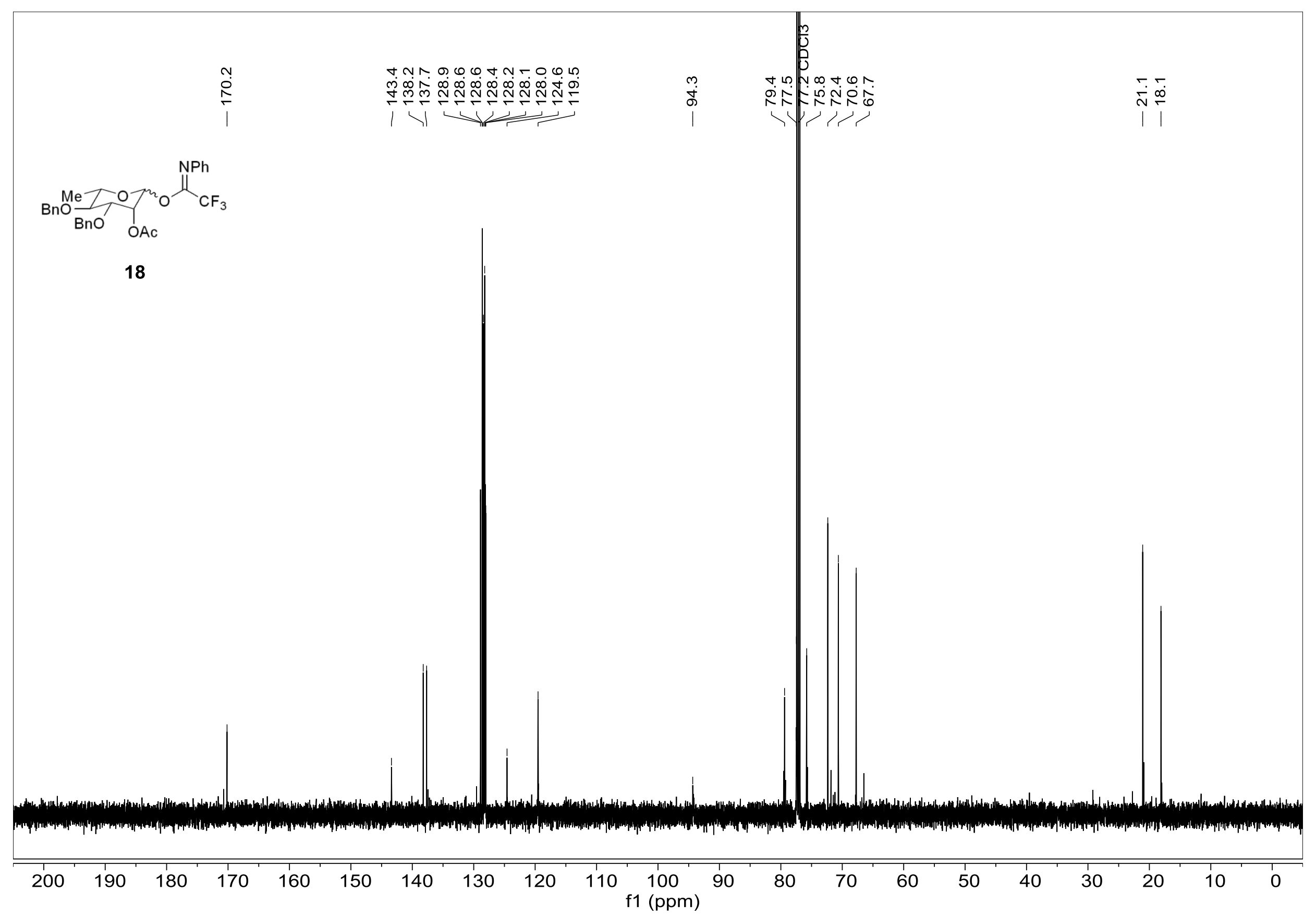

Figure S50. ${ }^{13} \mathrm{C}$ NMR spectrum of $\mathbf{1 8}\left(126 \mathrm{MHz}, \mathrm{CDCl}_{3}\right)$ 


$$
\mathrm{MeT}_{\mathrm{BnO}}^{\mathrm{Mn}} \mathrm{OAc}_{\mathrm{STol}}
$$

19

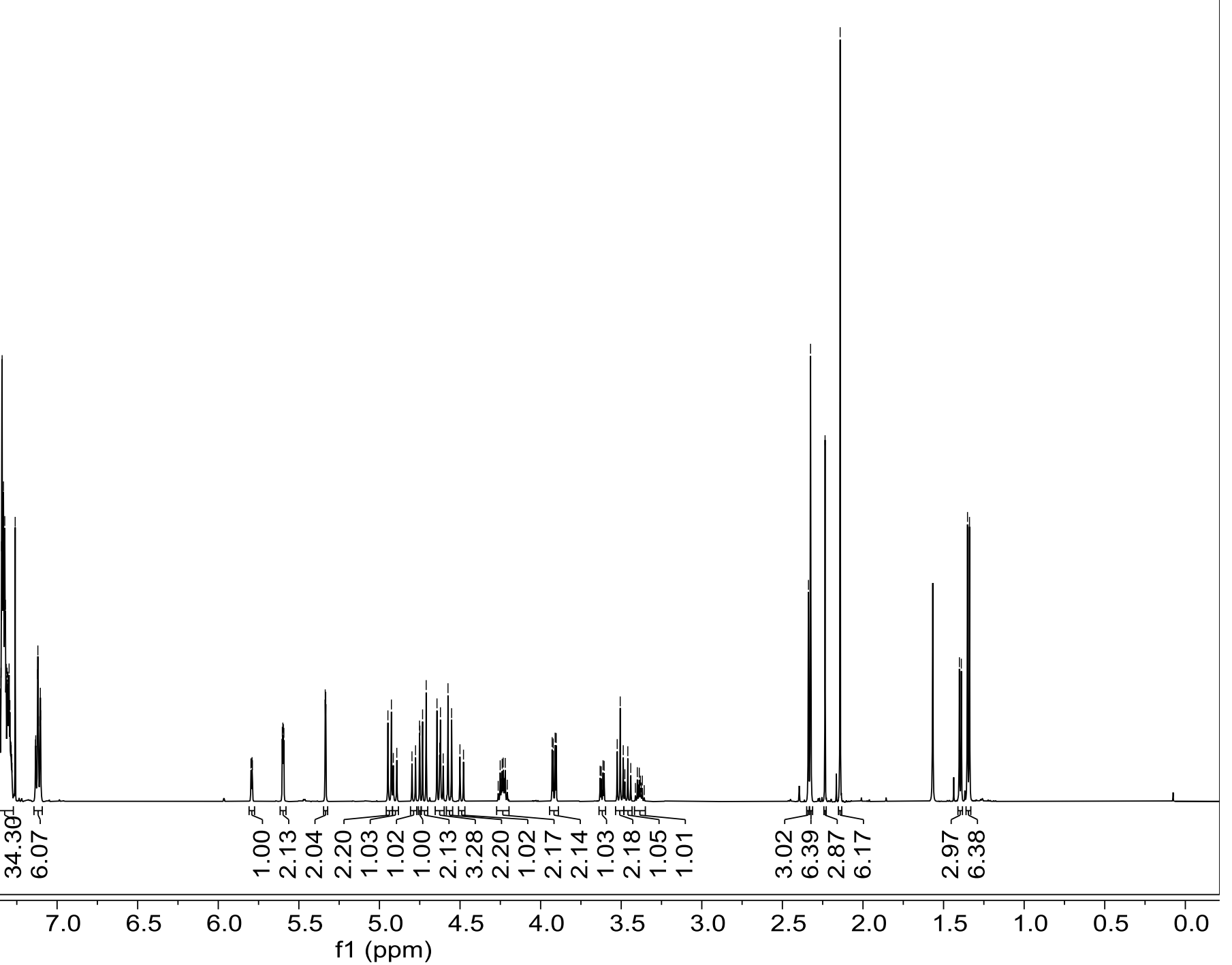

Figure S51. ${ }^{1} \mathrm{H}$ NMR spectrum of $19\left(500 \mathrm{MHz}, \mathrm{CDCl}_{3}\right)$ 


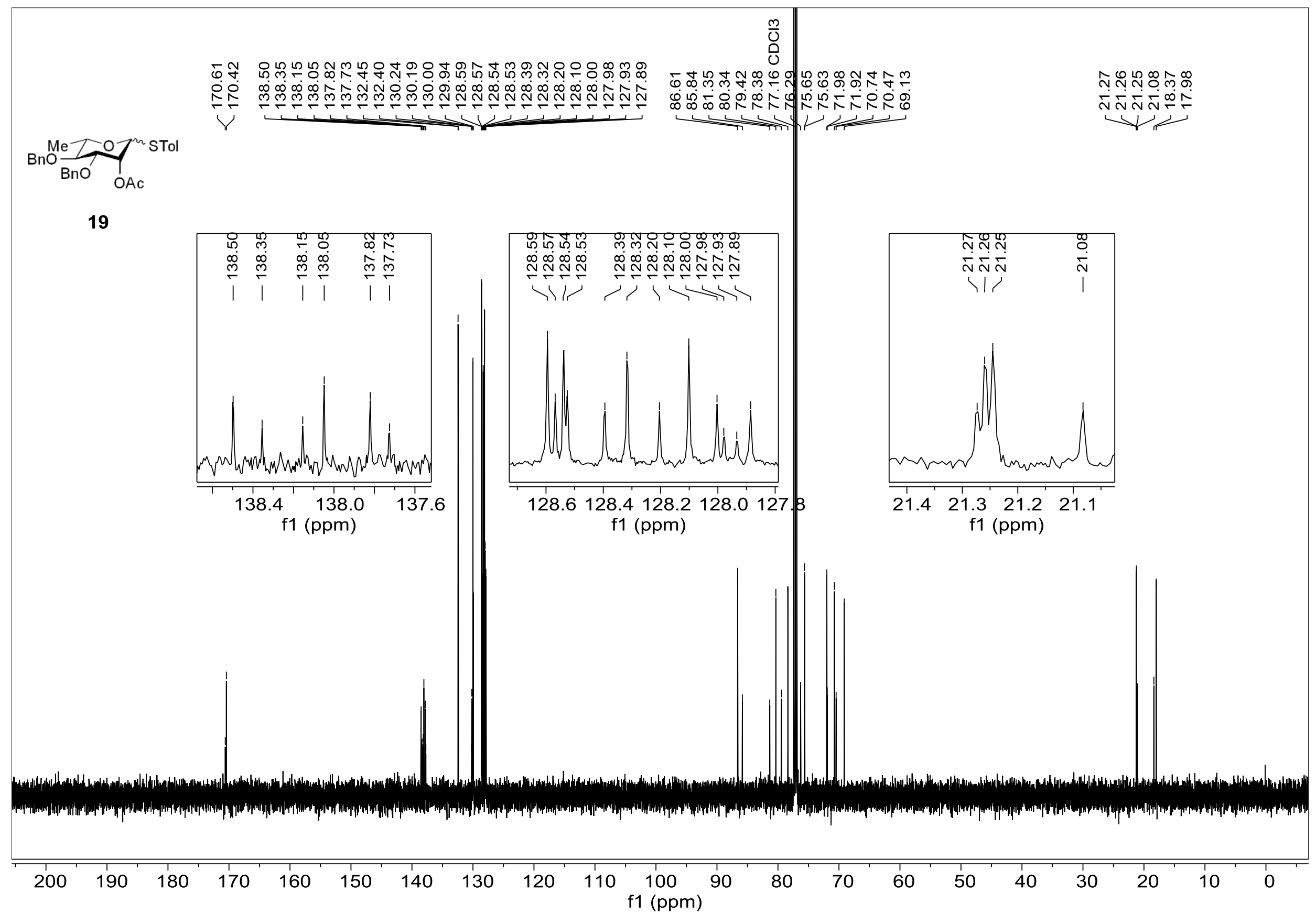

Figure S52. ${ }^{13} \mathrm{C}$ NMR spectrum of $\mathbf{1 9}\left(126 \mathrm{MHz}, \mathrm{CDCl}_{3}\right)$ 


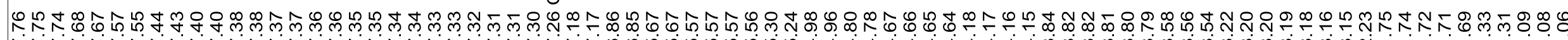

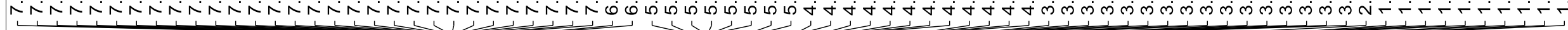
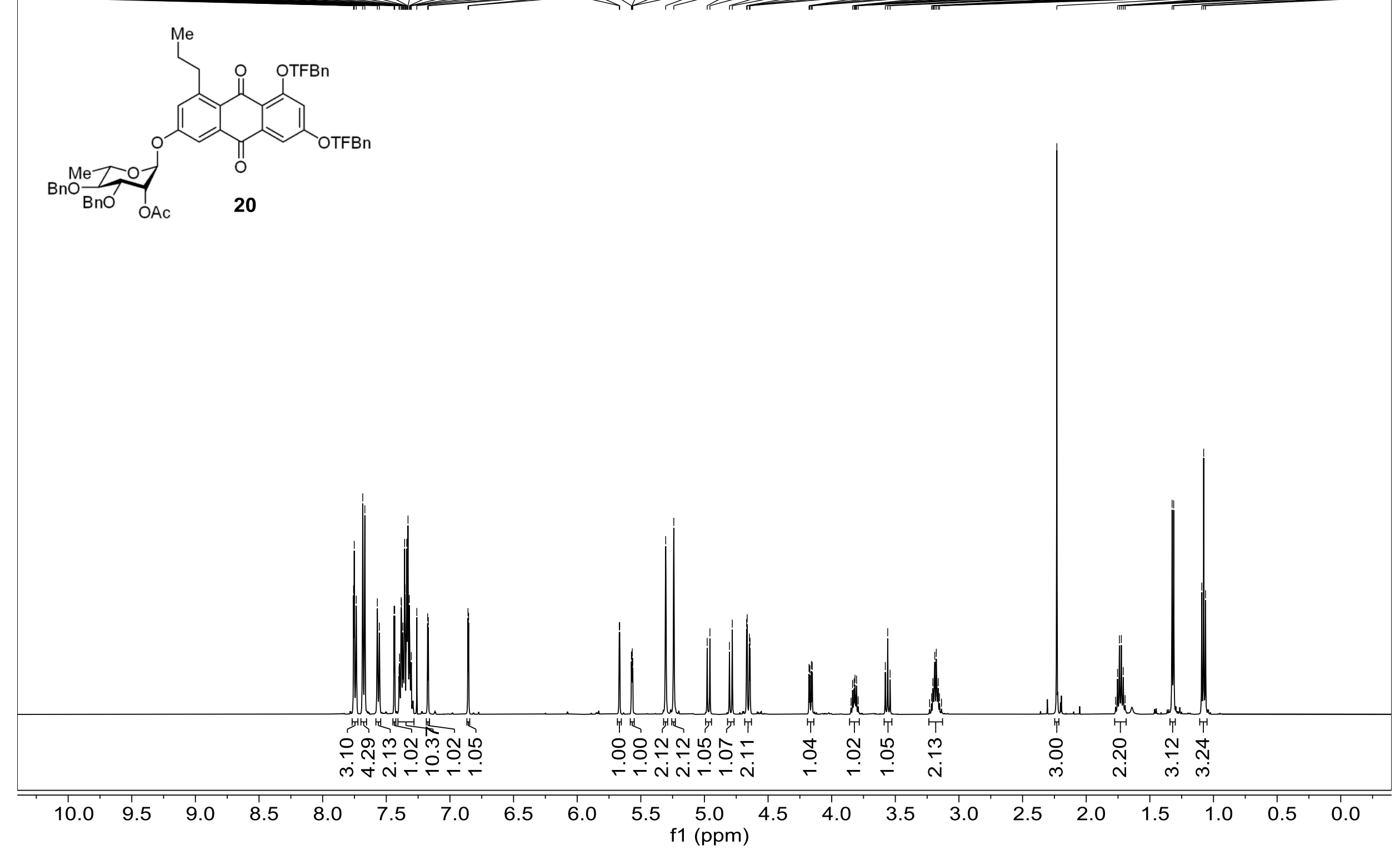

Figure S53. ${ }^{1} \mathrm{H}$ NMR spectrum of $20\left(500 \mathrm{MHz}, \mathrm{CDCl}_{3}\right)$ 


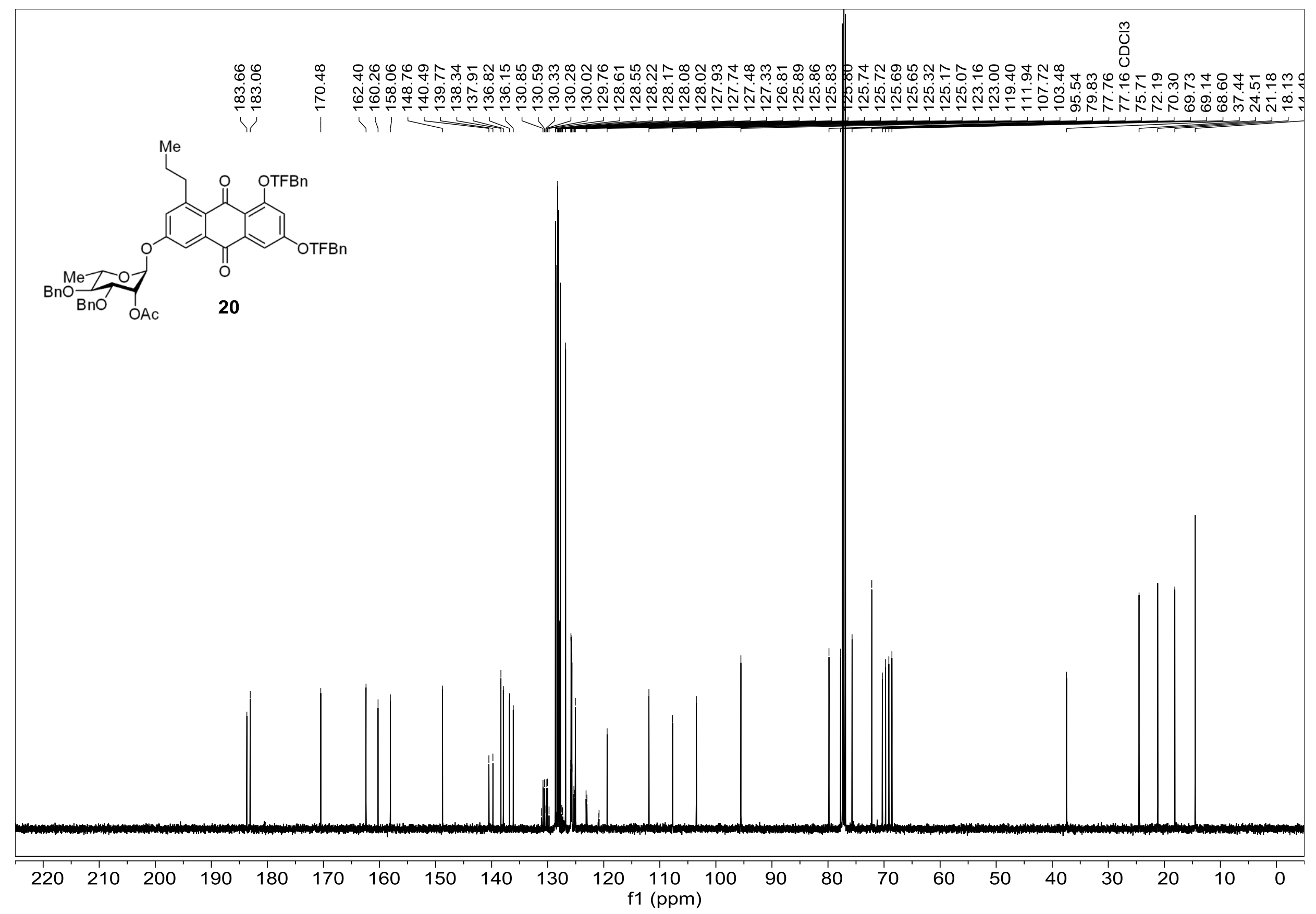

Figure S54. ${ }^{13} \mathrm{C}$ NMR spectrum of $20\left(126 \mathrm{MHz}, \mathrm{CDCl}_{3}\right)$ 


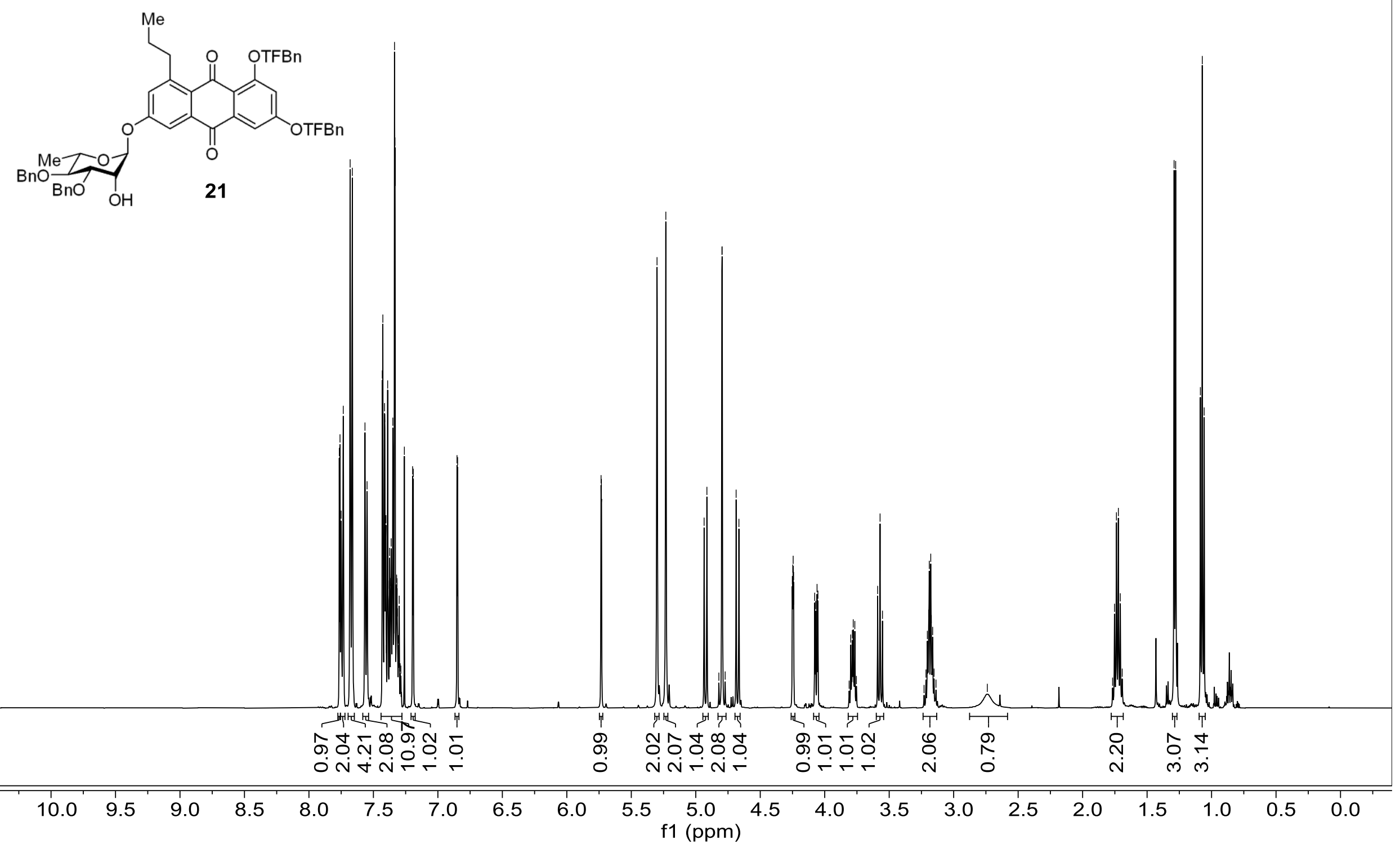

Figure S55. ${ }^{1} \mathrm{H}$ NMR spectrum of $21\left(500 \mathrm{MHz}, \mathrm{CDCl}_{3}\right)$ 


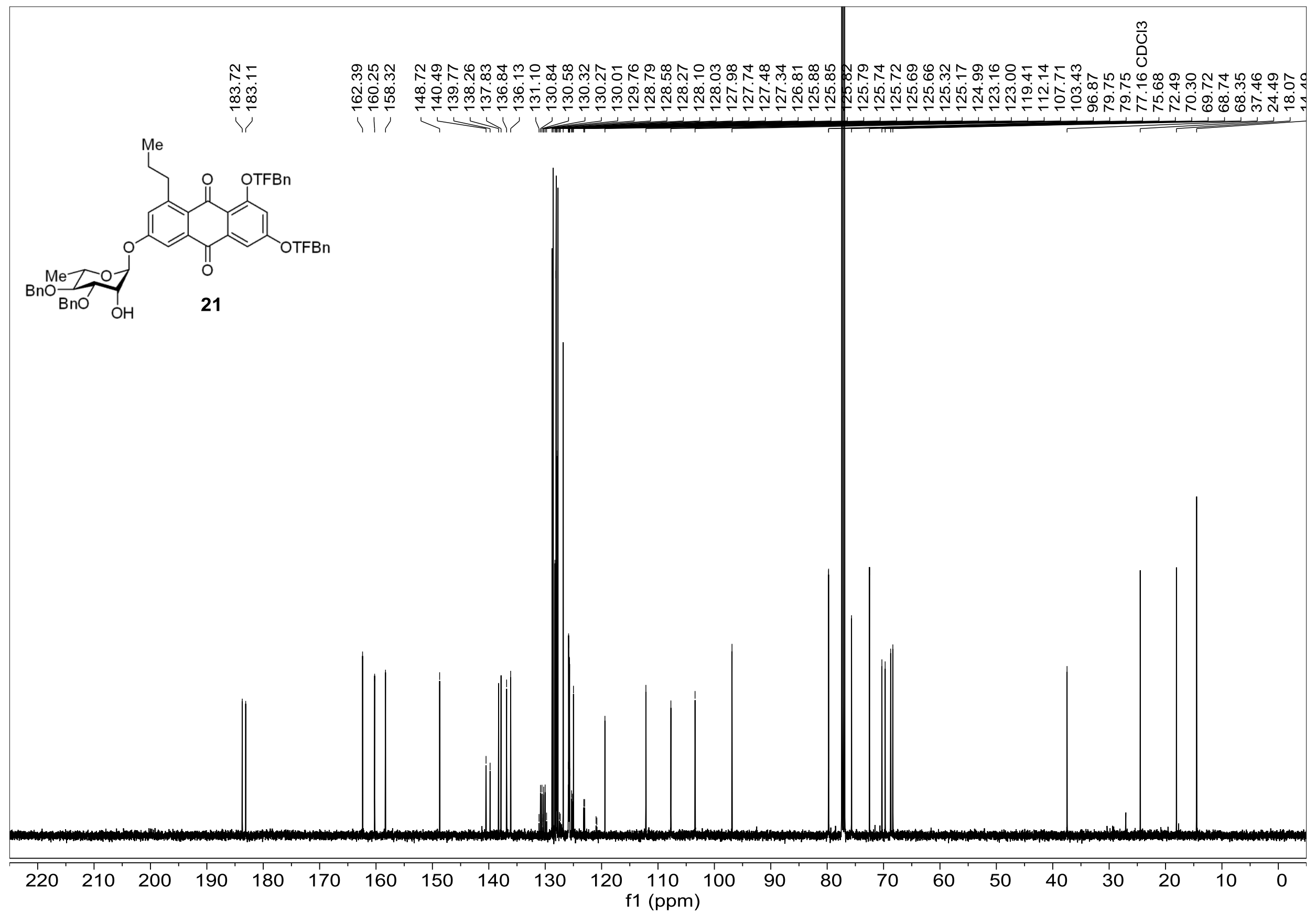

Figure S56. ${ }^{13} \mathrm{C}$ NMR spectrum of $21\left(126 \mathrm{MHz}, \mathrm{CDCl}_{3}\right)$ 


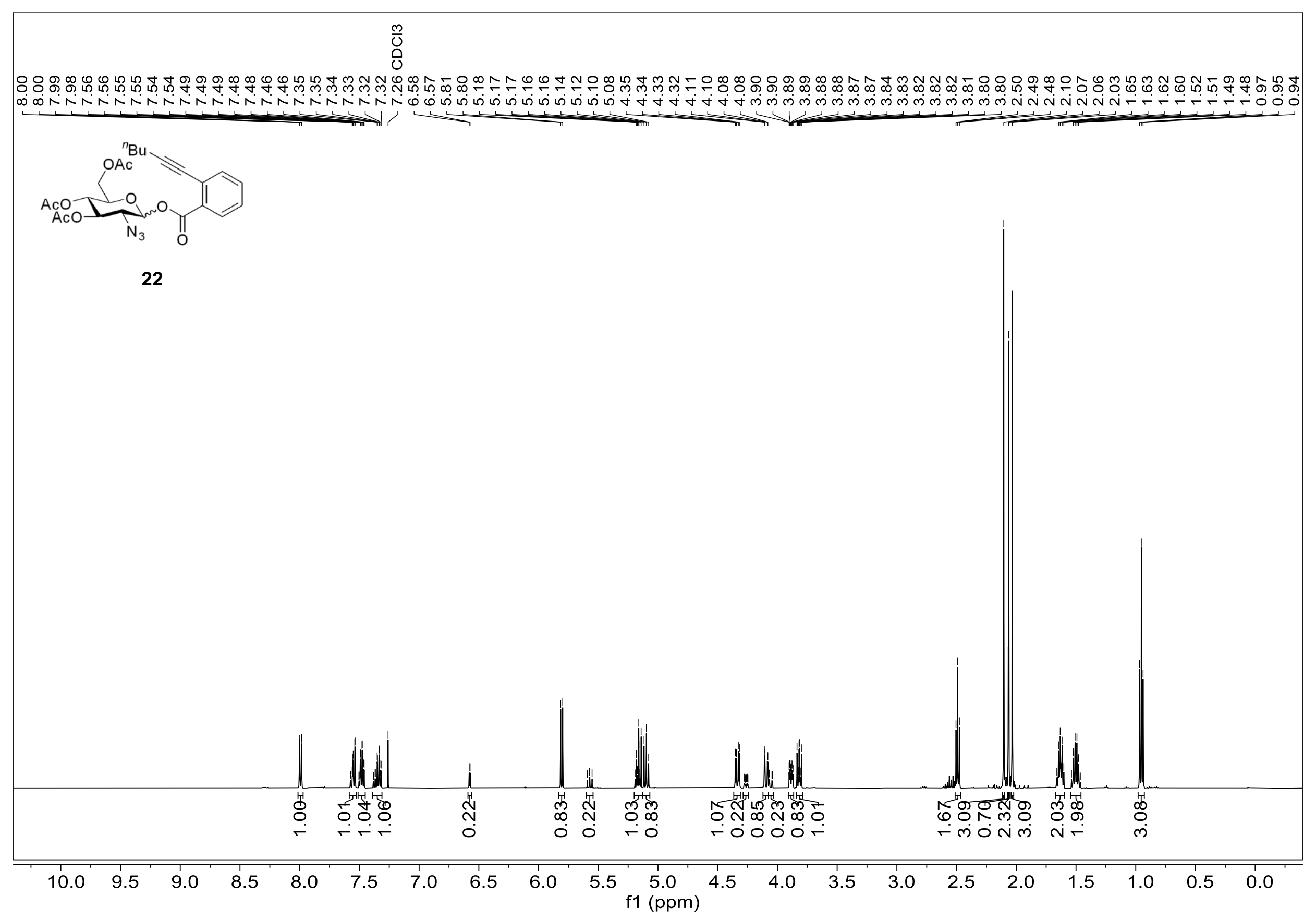

Figure S57. ${ }^{1} \mathrm{H}$ NMR spectrum of $22\left(500 \mathrm{MHz}, \mathrm{CDCl}_{3}\right)$ 


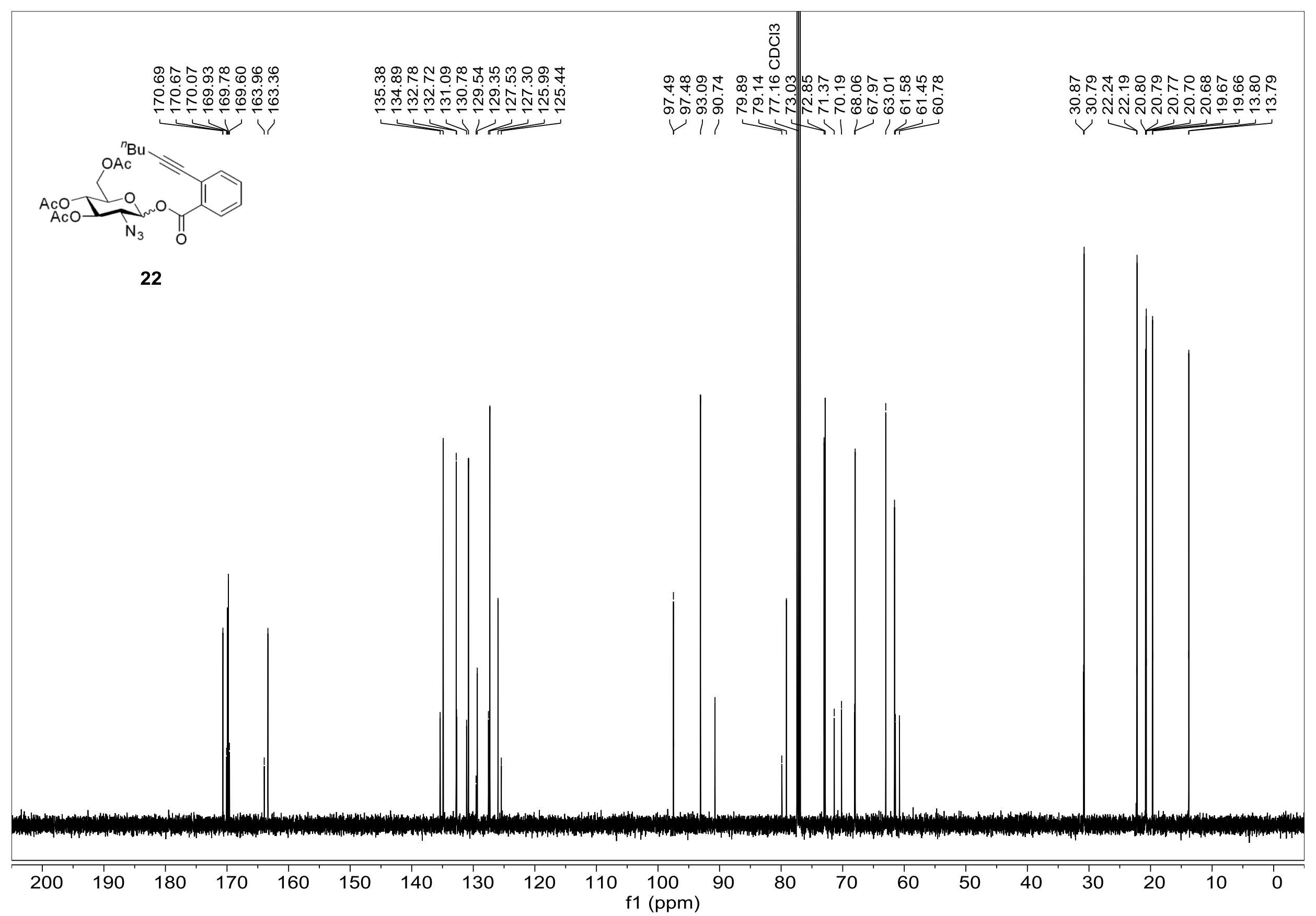

Figure S58. ${ }^{13} \mathrm{C}$ NMR spectrum of $22\left(126 \mathrm{MHz}, \mathrm{CDCl}_{3}\right)$ 


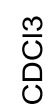

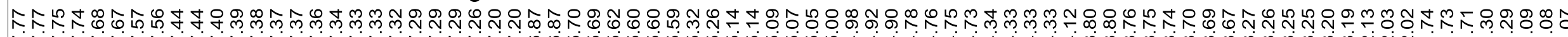

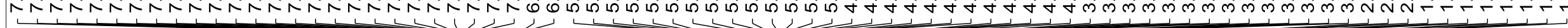

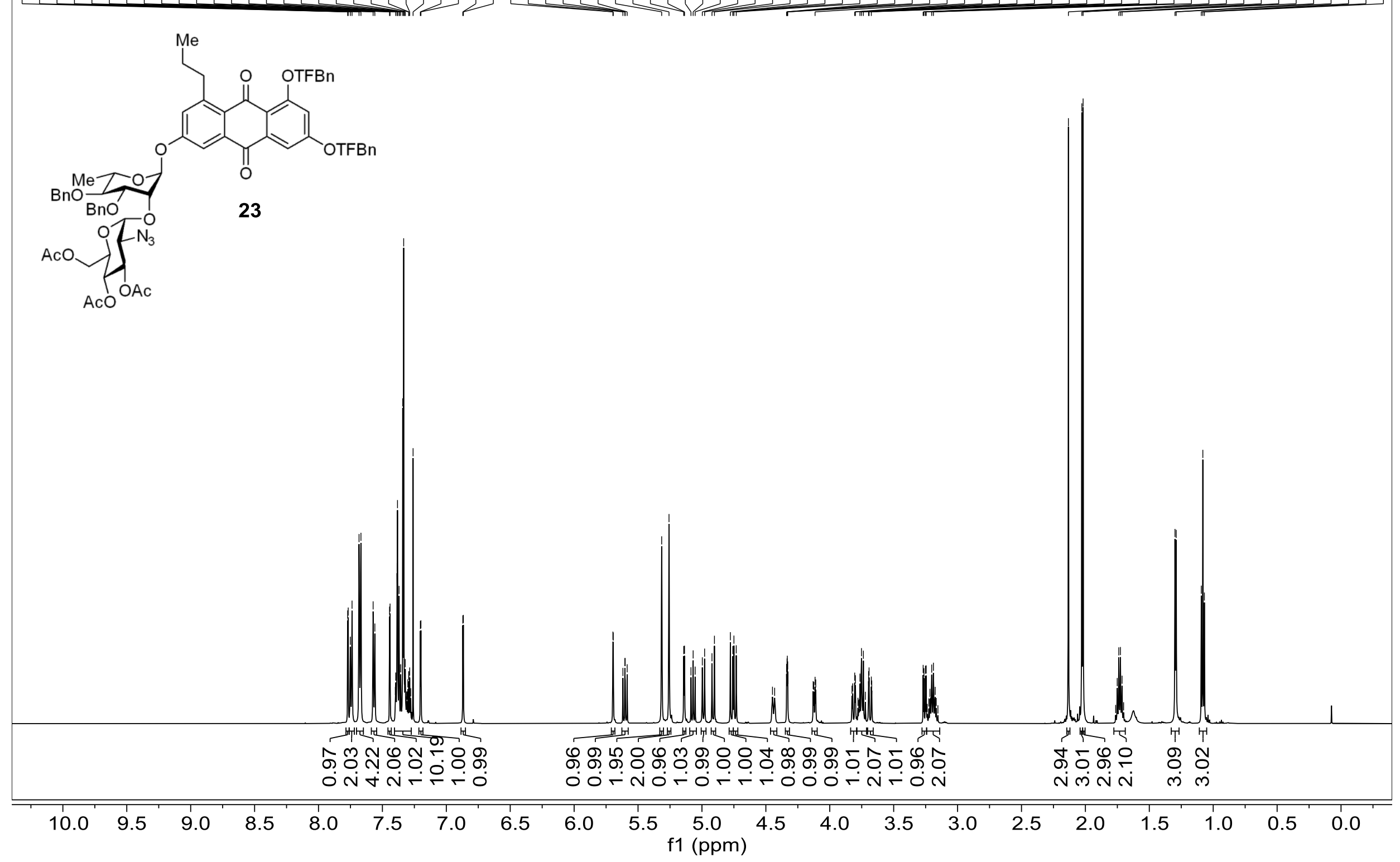

Figure S59. ${ }^{1} \mathrm{H}$ NMR spectrum of $23\left(600 \mathrm{MHz}, \mathrm{CDCl}_{3}\right)$ 


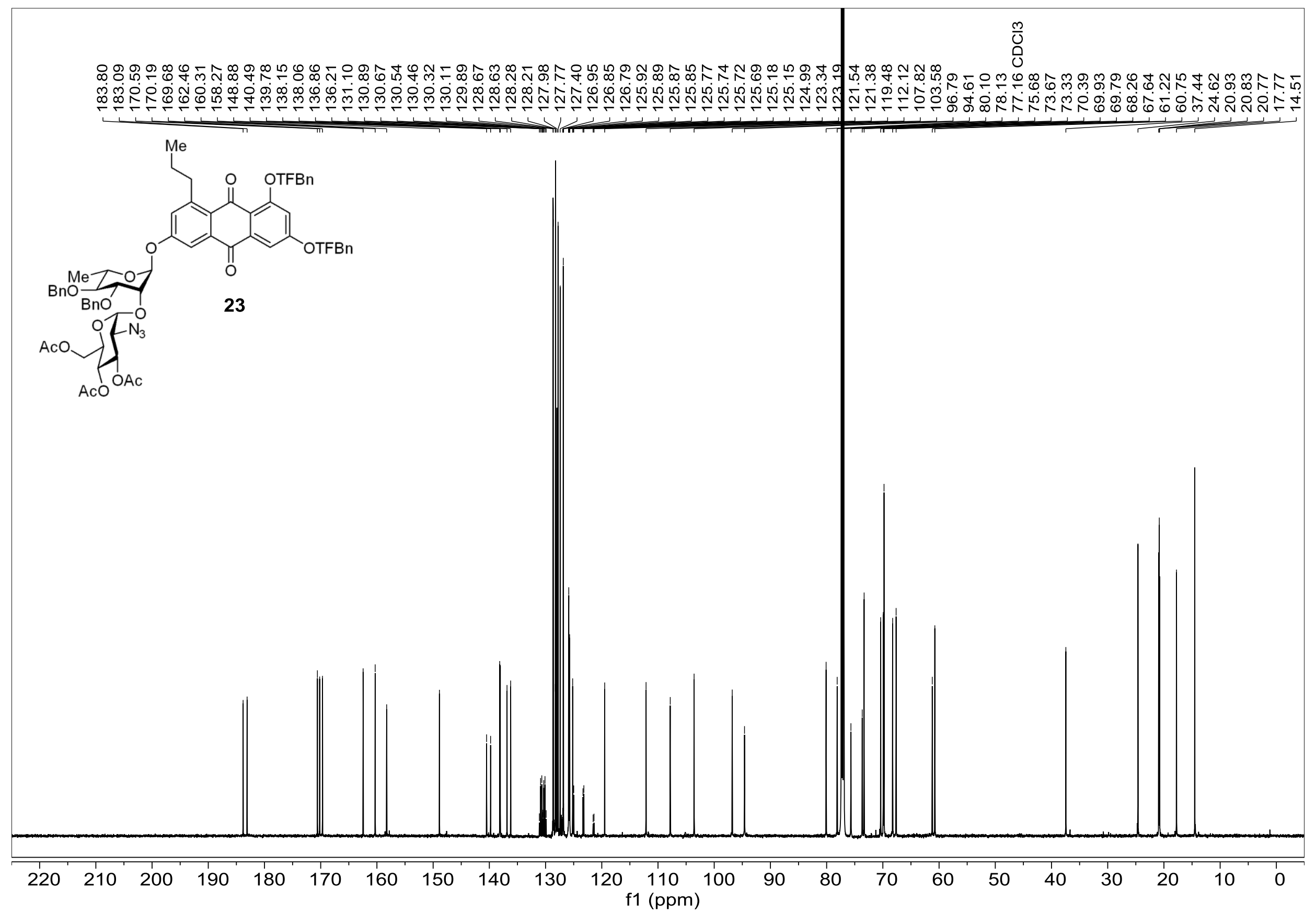

Figure S60. ${ }^{13} \mathrm{C}$ NMR spectrum of $23\left(151 \mathrm{MHz}, \mathrm{CDCl}_{3}\right)$ 


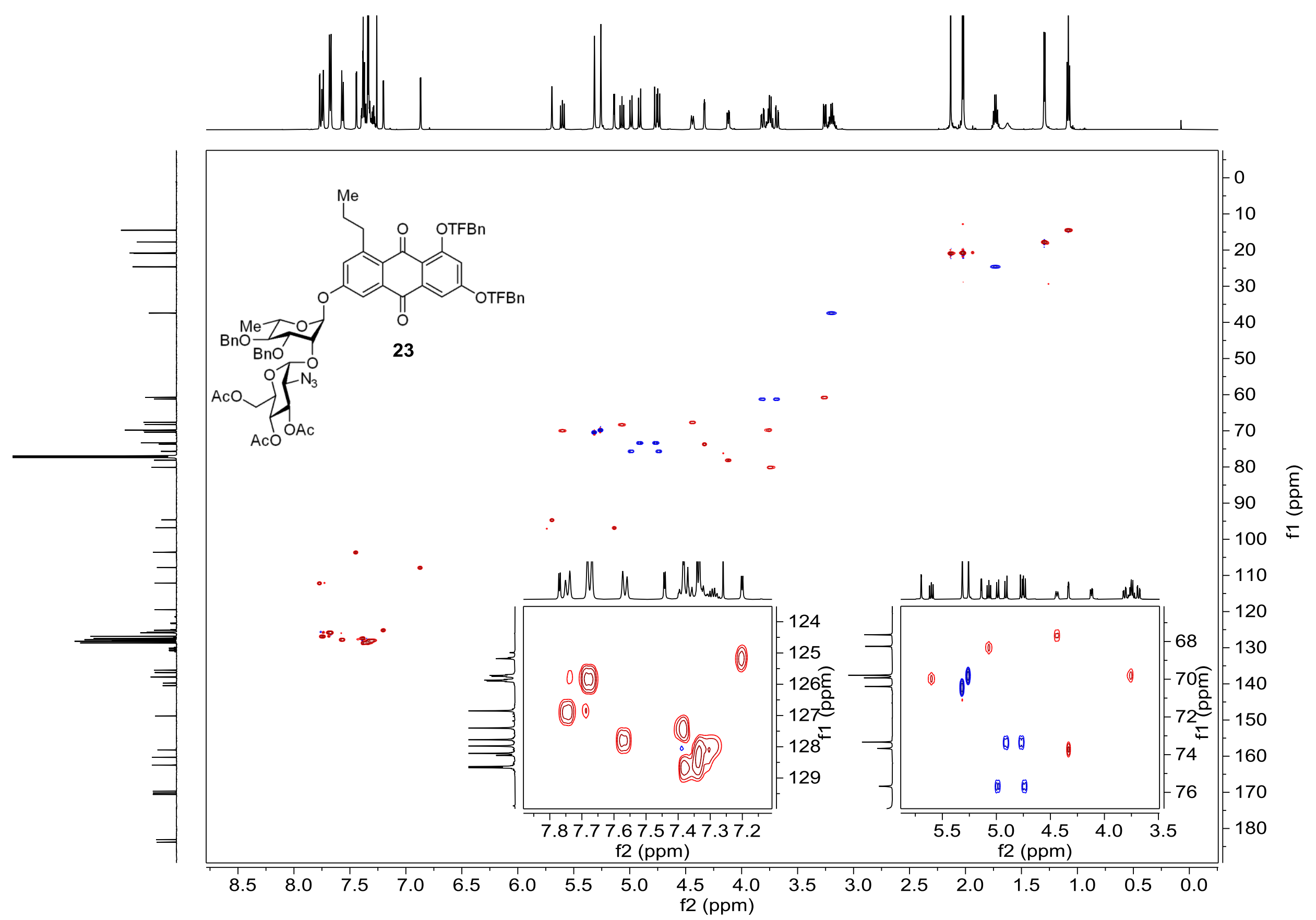

Figure S61. HSQC spectrum of 23 in $\mathrm{CDCl}_{3}$ 


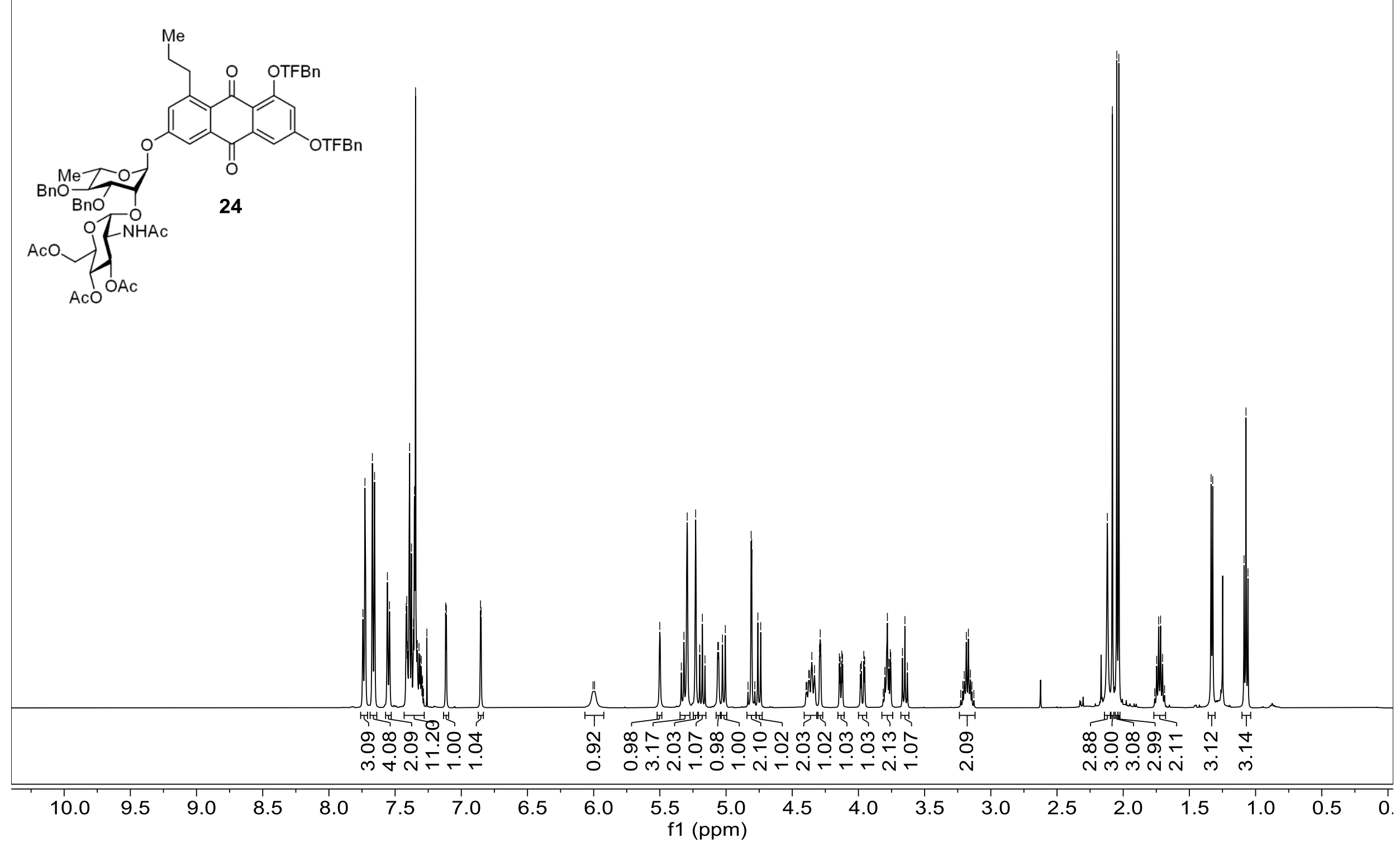

Figure S62. ${ }^{1} \mathrm{H}$ NMR spectrum of $24\left(500 \mathrm{MHz}, \mathrm{CDCl}_{3}\right)$ 

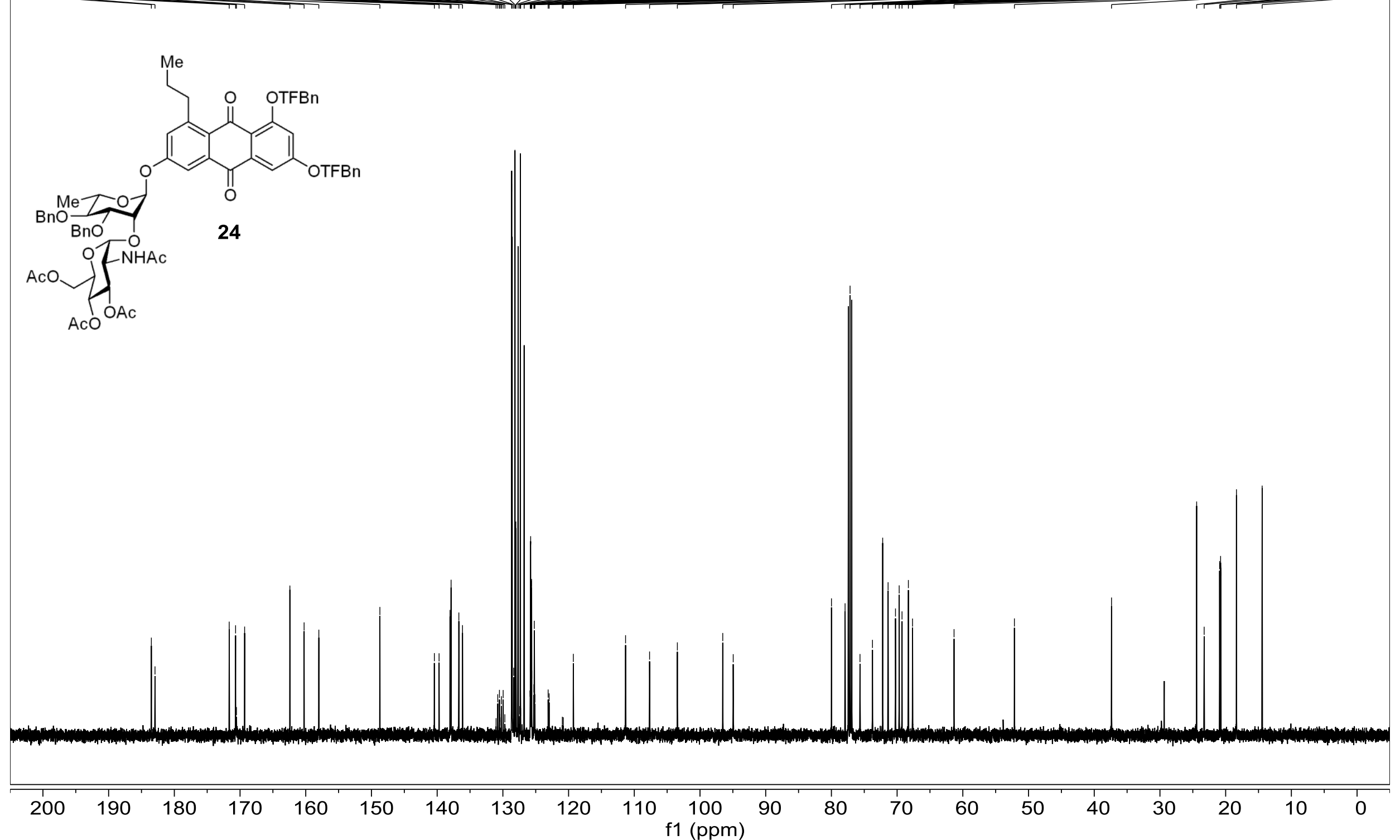

Figure S63. ${ }^{13} \mathrm{C}$ NMR spectrum of $24\left(126 \mathrm{MHz}, \mathrm{CDCl}_{3}\right)$ 


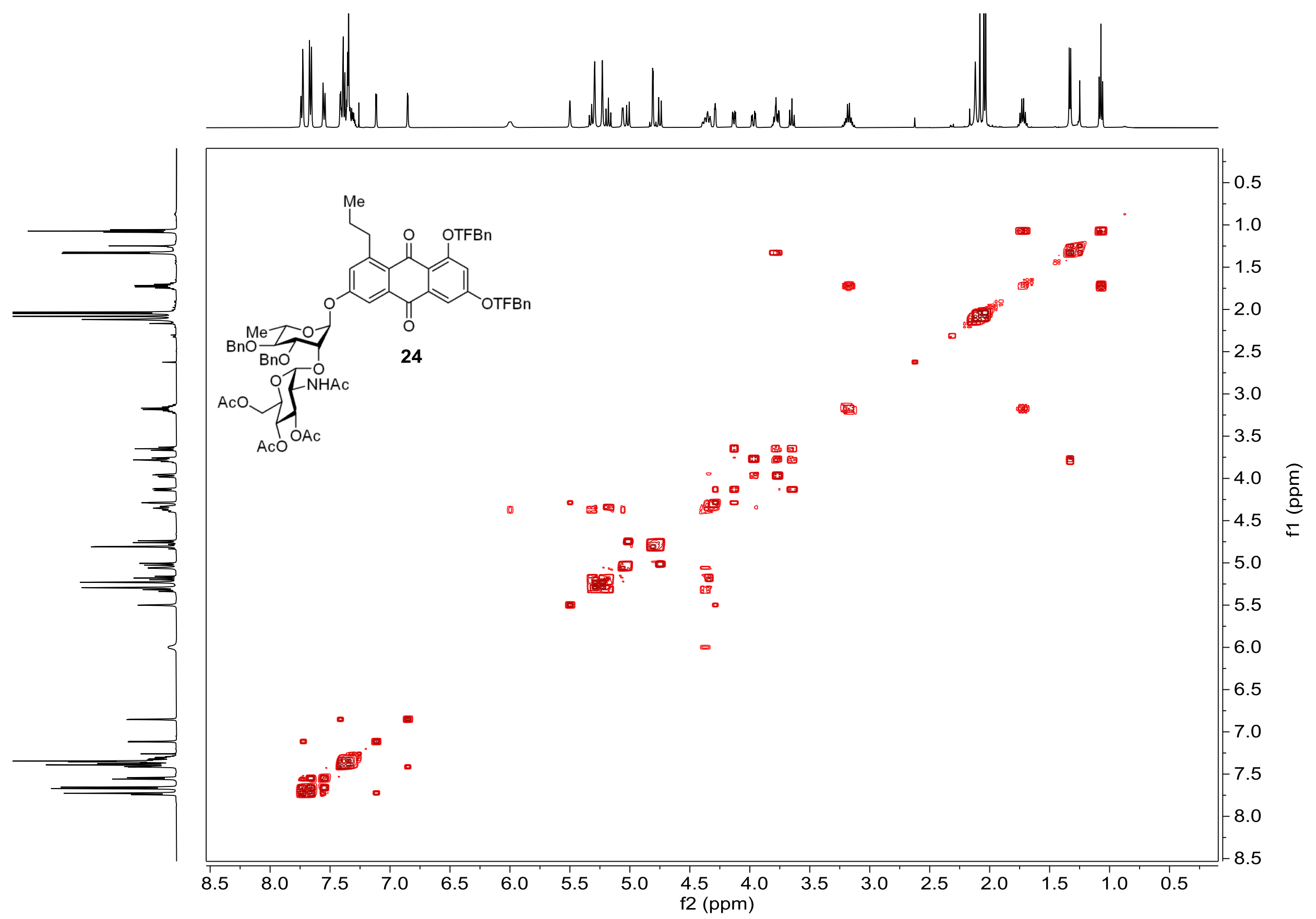

Figure S64. COSY spectrum of 24 in $\mathrm{CDCl}_{3}$ 


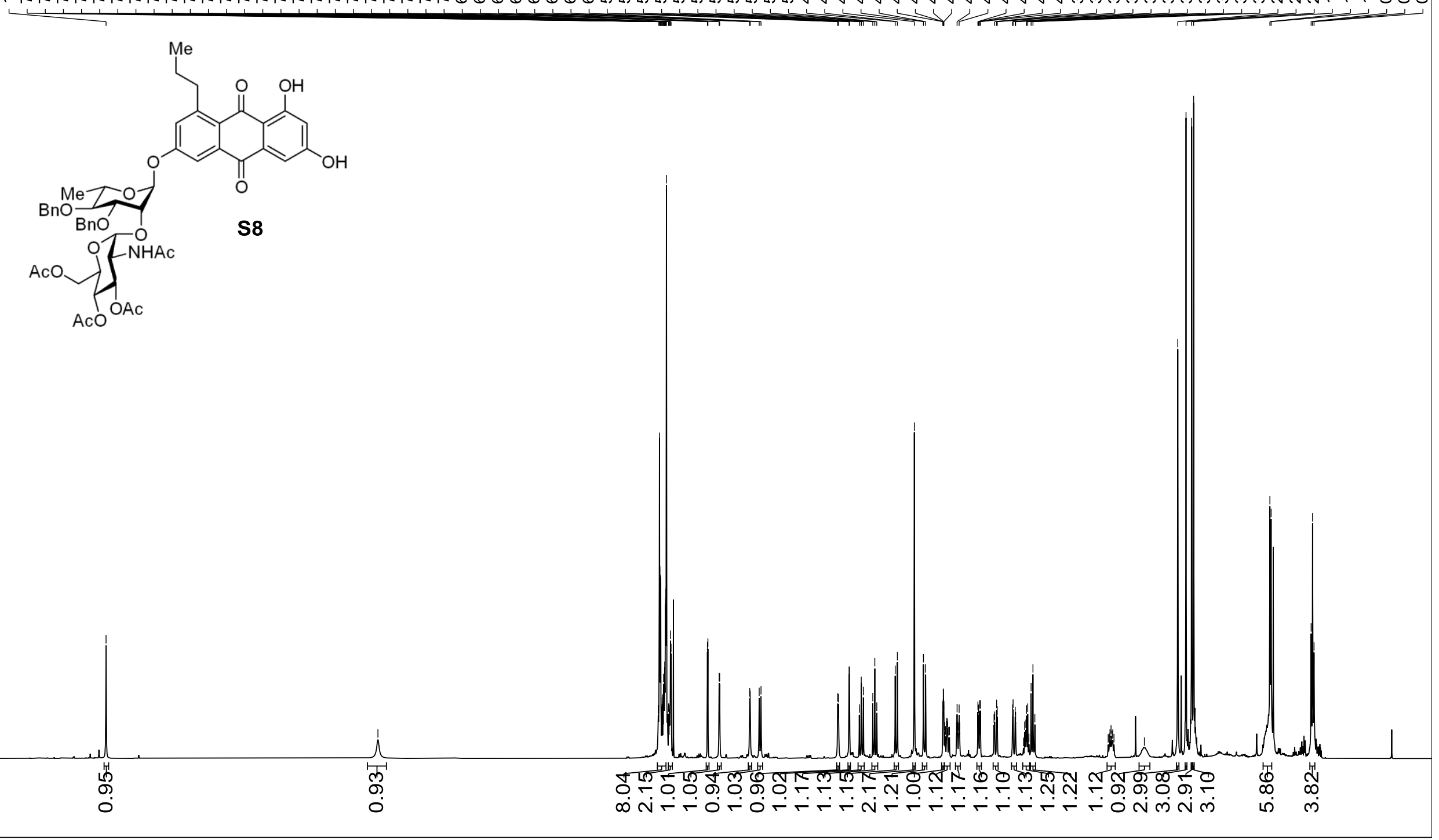

$\begin{array}{lllllllllllllllllllllllllllllllllllllllll}13.5 & 13.0 & 12.5 & 12.0 & 11.5 & 11.0 & 10.5 & 10.0 & 9.5 & 9.0 & 8.5 & 8.0 & 7.5 & 7.0 & 6.5 & 6.0 & 5.5 & 5.0 & 4.5 & 4.0 & 3.5 & 3.0 & 2.5 & 2.0 & 1.5 & 1.0 & 0.5 & 0.0\end{array}$

Figure S65. ${ }^{1} \mathrm{H}$ NMR spectrum of $\mathbf{S 8}\left(500 \mathrm{MHz}, \mathrm{CDCl}_{3}\right)$ 


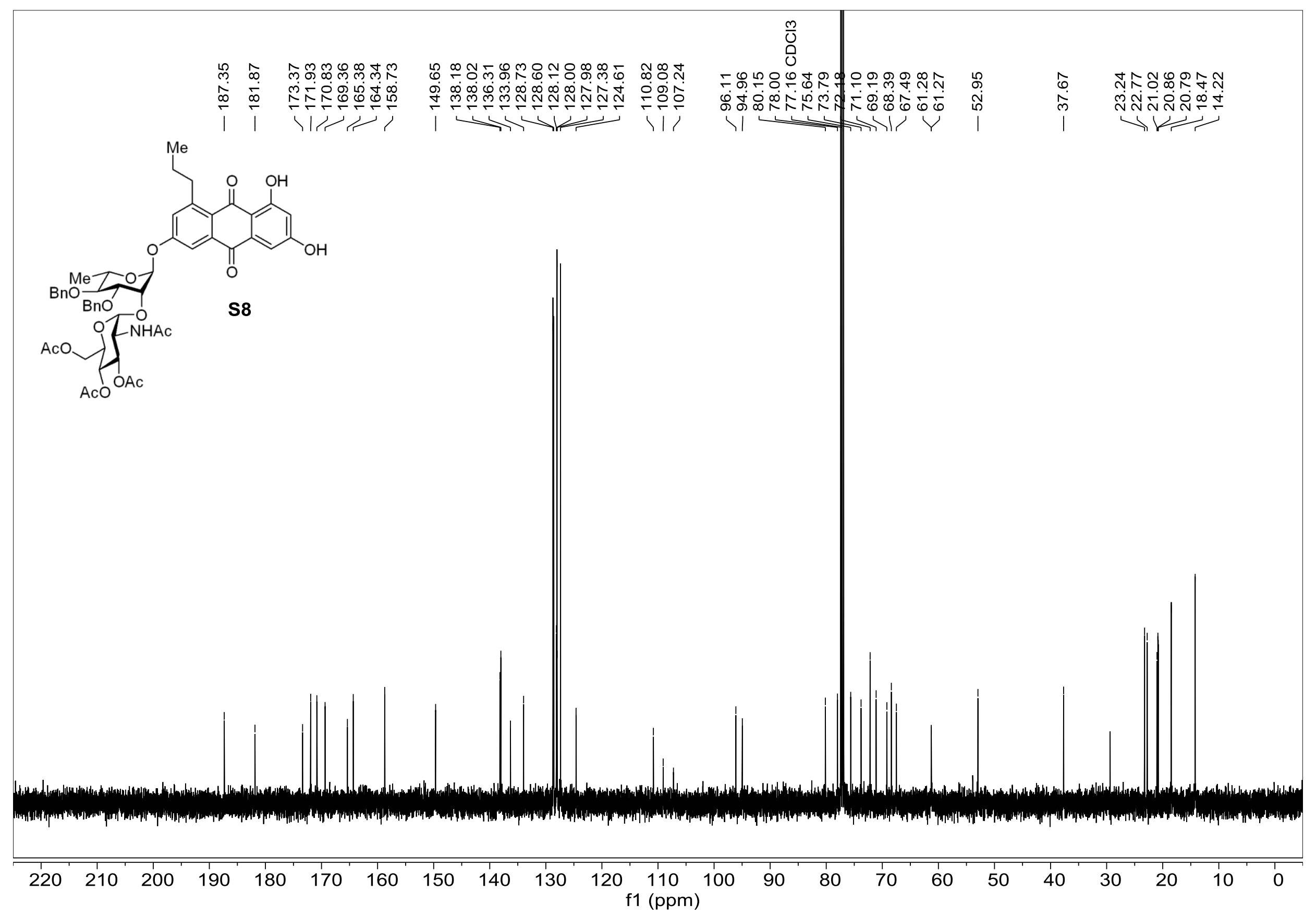

Figure S66. ${ }^{13} \mathrm{C}$ NMR spectrum of $\mathbf{S 8}\left(126 \mathrm{MHz}, \mathrm{CDCl}_{3}\right)$ 


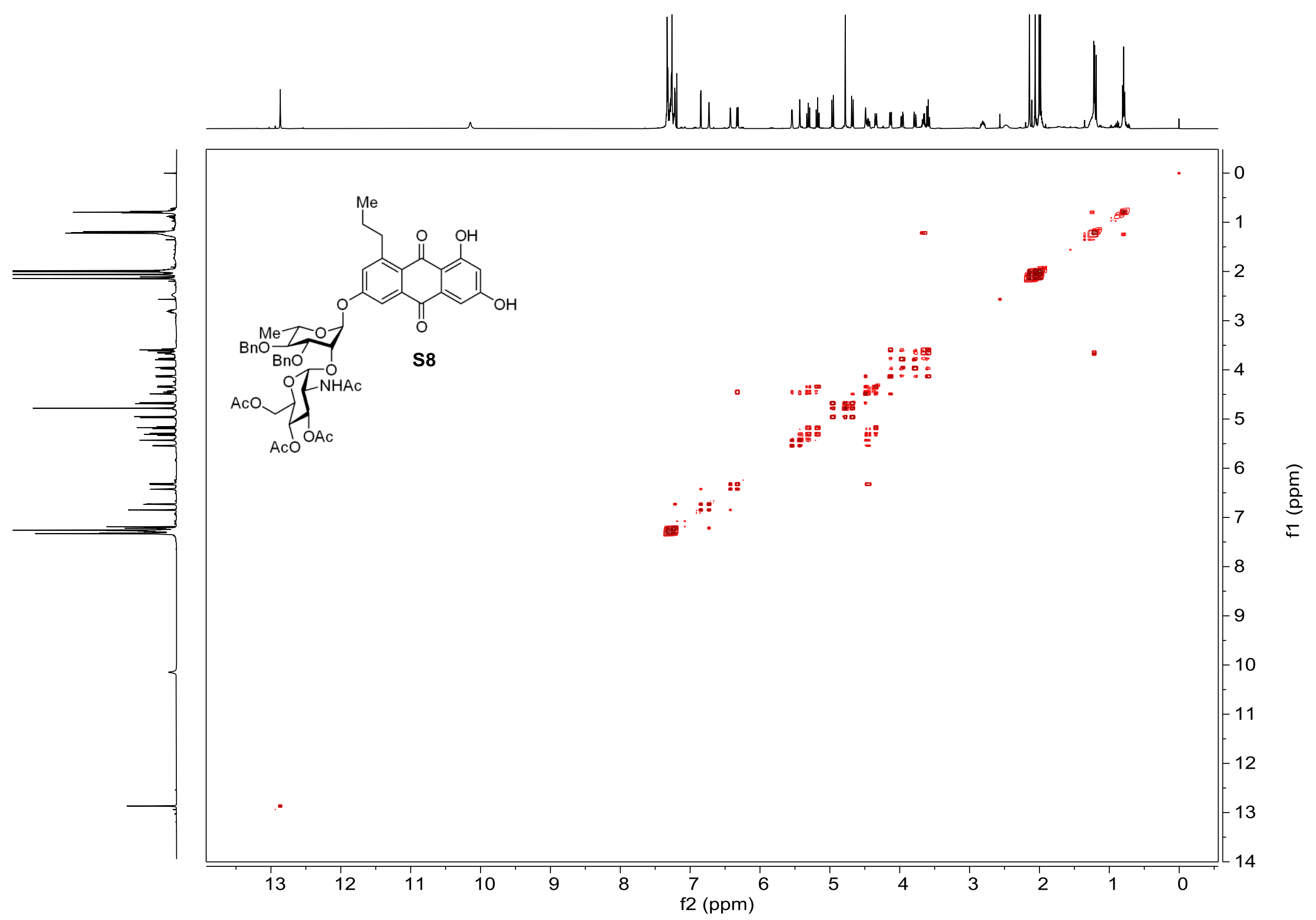

Figure S67. COSY spectrum of $\mathbf{S 8}$ in $\mathrm{CDCl}_{3}$ 

等

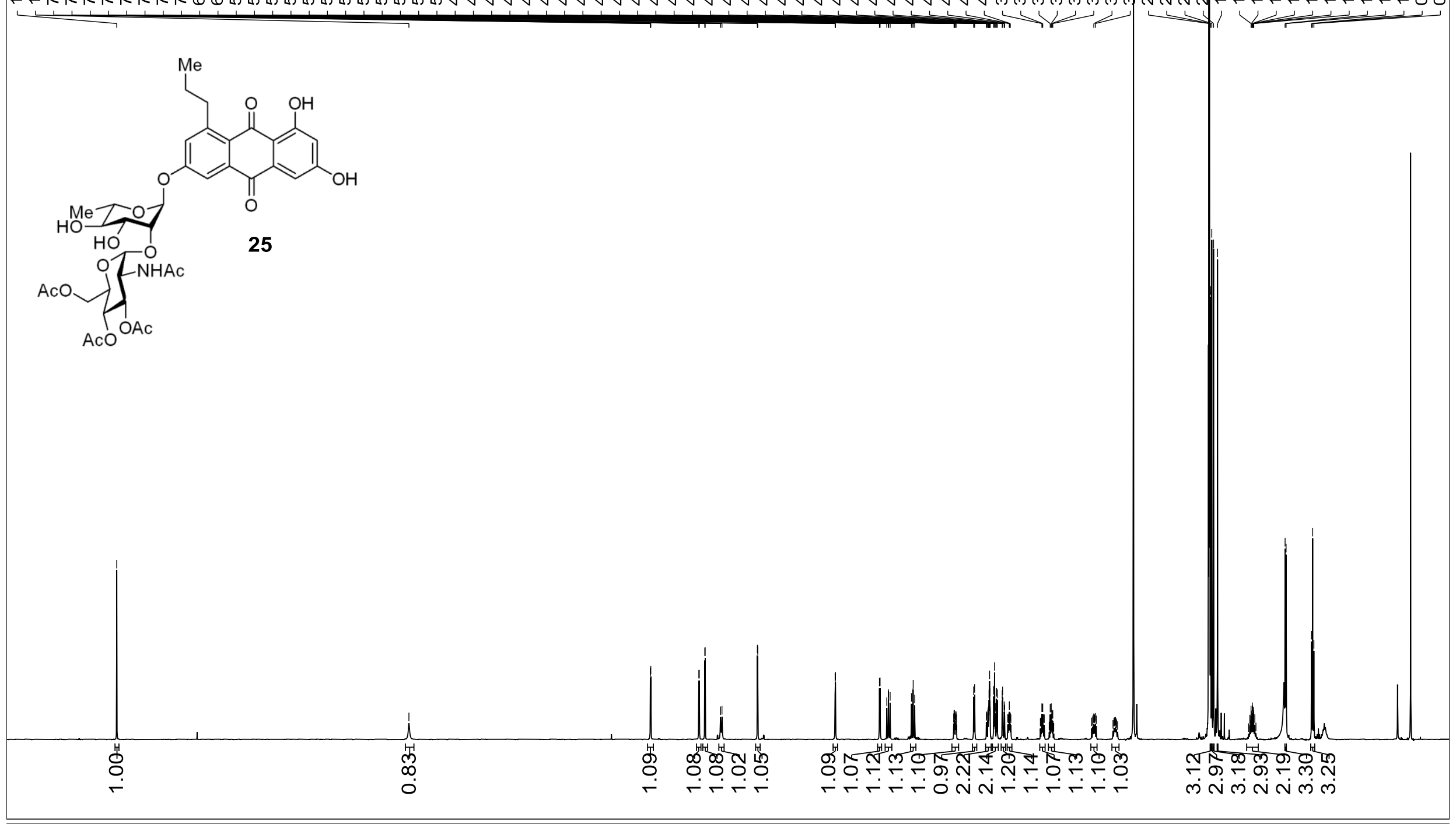

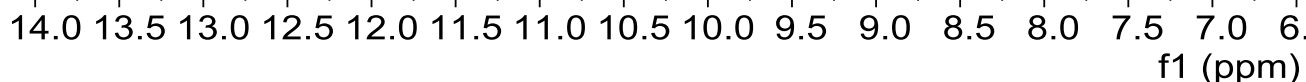

Figure S68. ${ }^{1} \mathrm{H}$ NMR spectrum of 25 (600 MHz, acetone- $\left.d_{6}\right)$ 


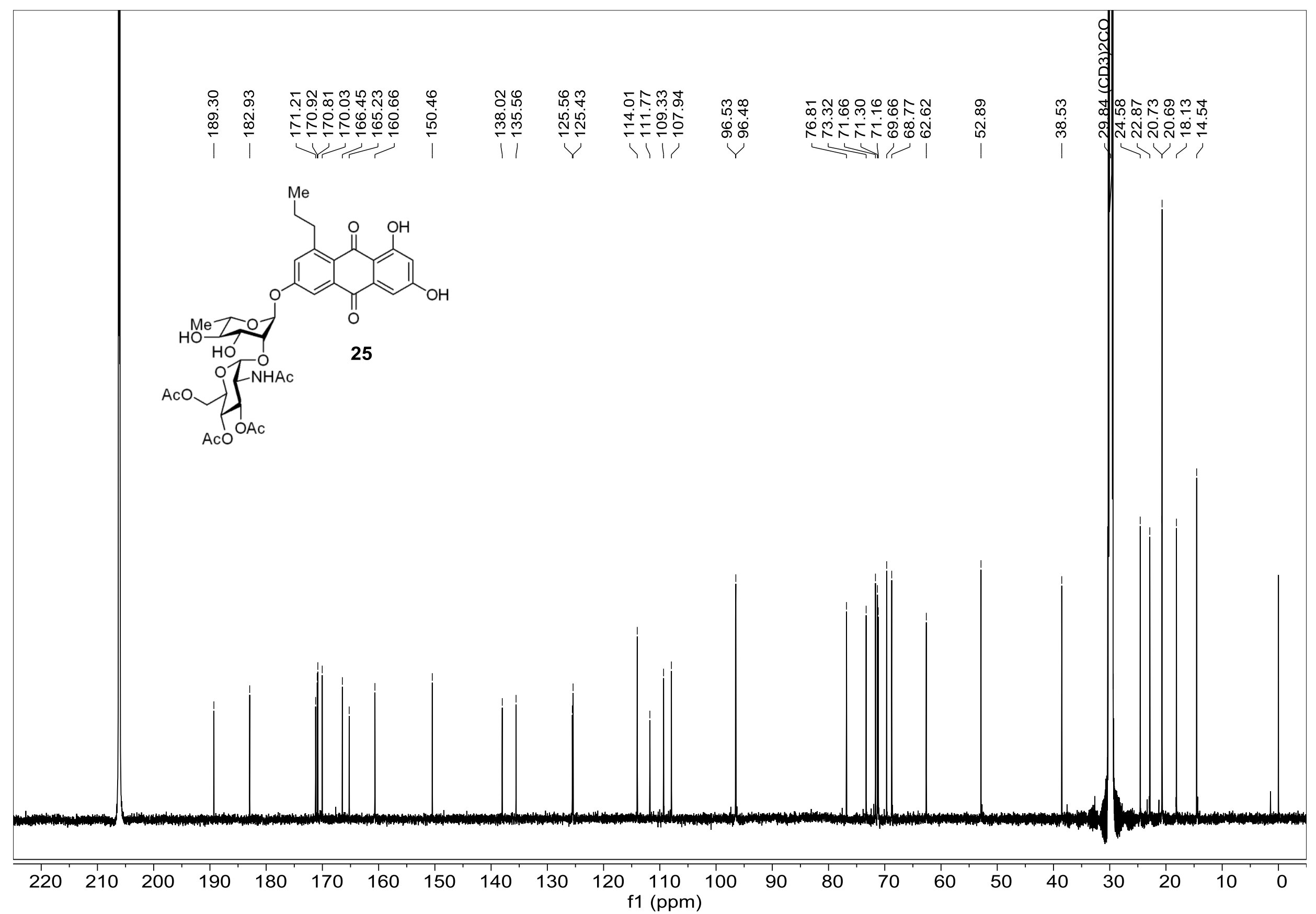

Figure S69. ${ }^{13} \mathrm{C}$ NMR spectrum of 25 (151 MHz, acetone- $d_{6}$ ) 


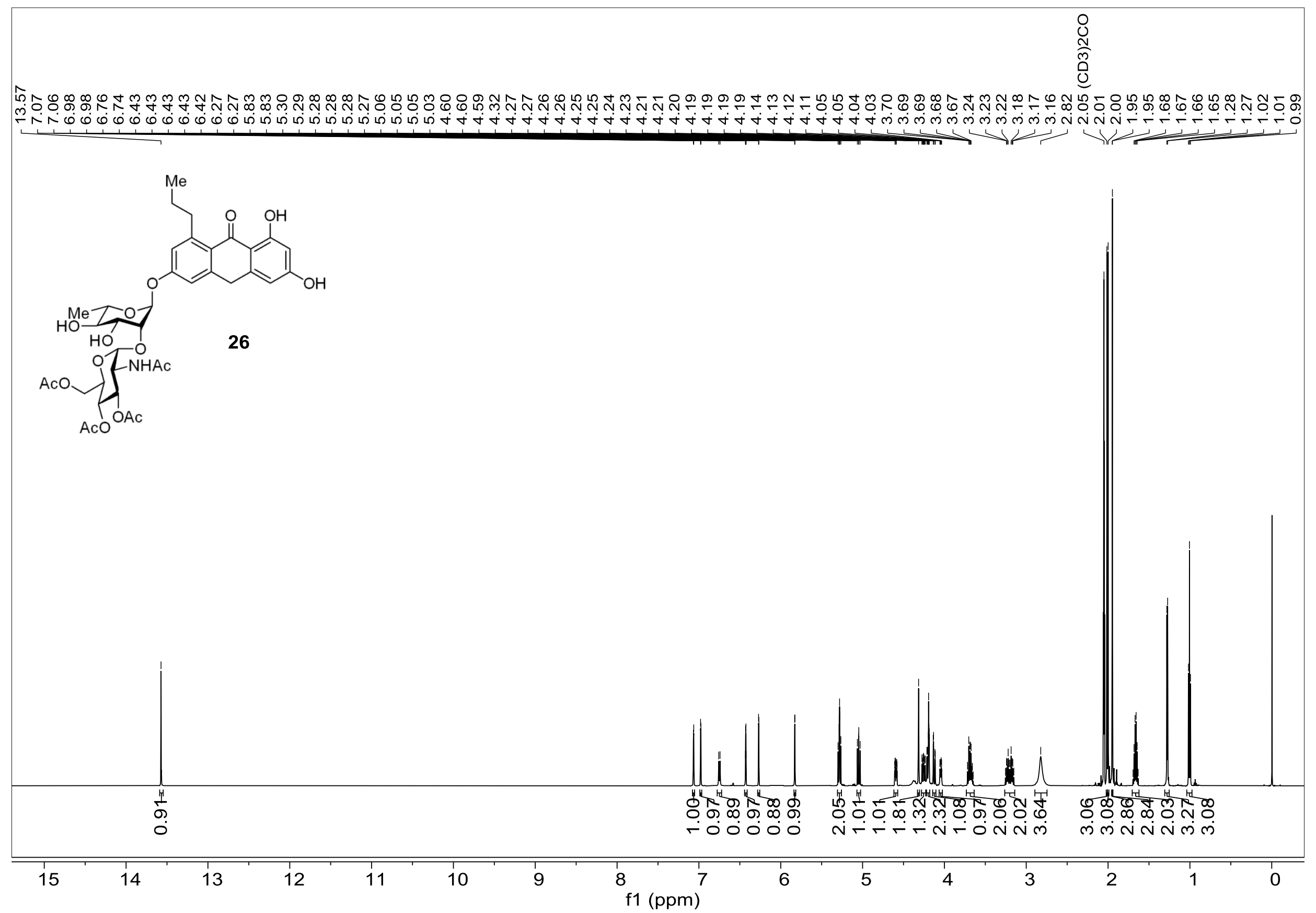

Figure S70. ${ }^{1} \mathrm{H}$ NMR spectrum of $26\left(600 \mathrm{MHz}\right.$, acetone- $\left.d_{6}\right)$ 


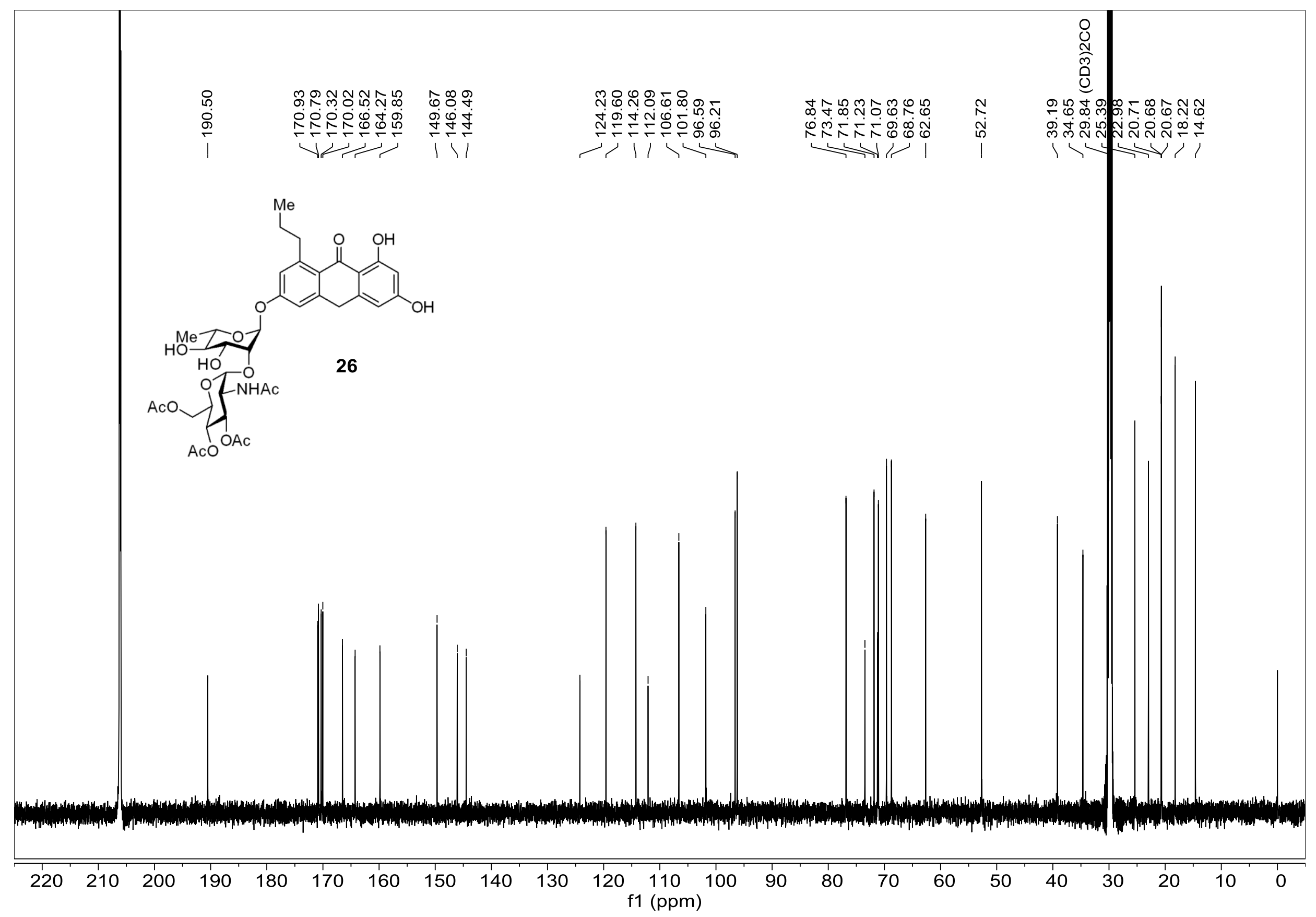

Figure S71. ${ }^{13} \mathrm{C}$ NMR spectrum of $26\left(151 \mathrm{MHz}\right.$, acetone- $\left.d_{6}\right)$ 


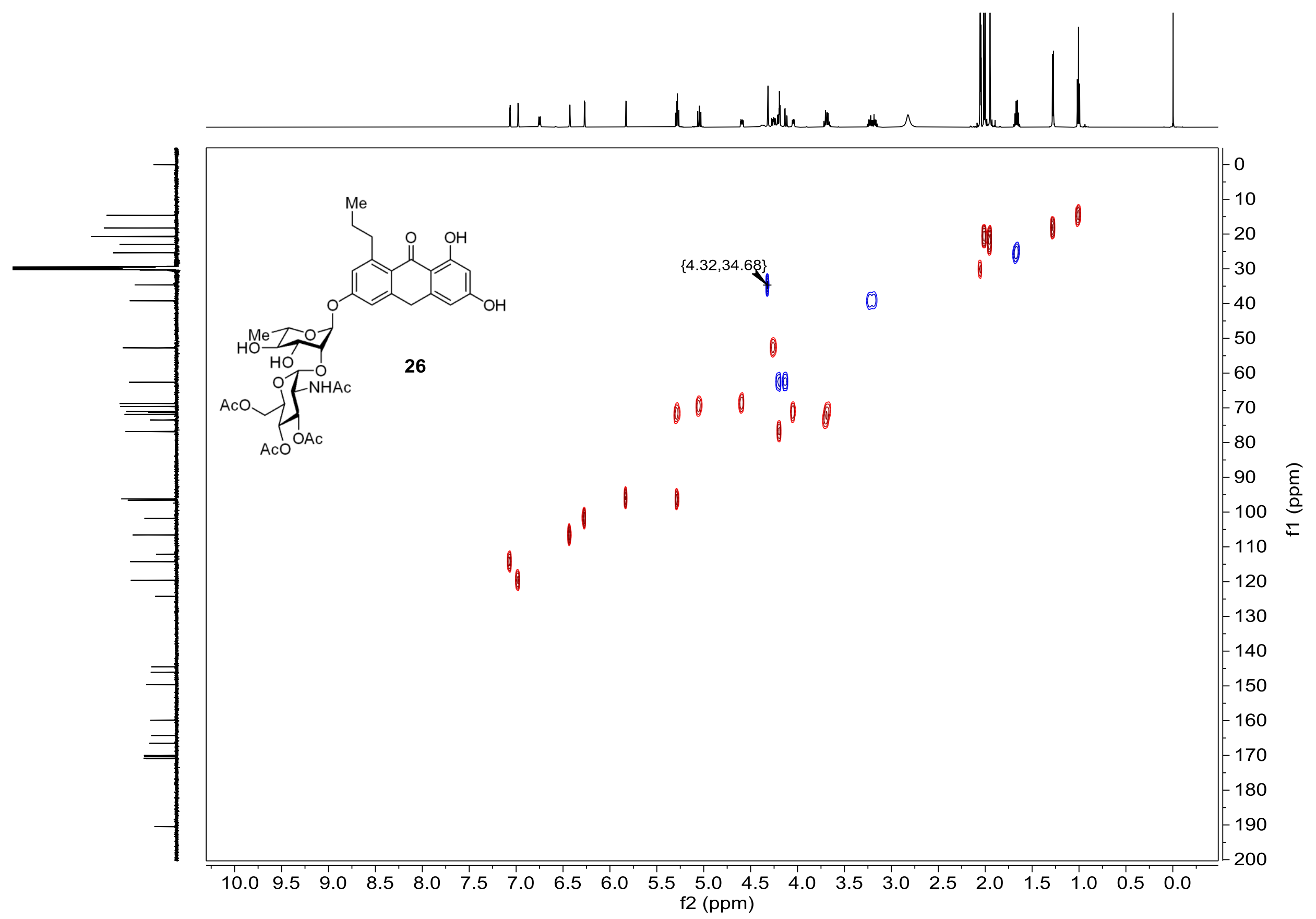

Figure S72. HSQC spectrum of $\mathbf{2 6}$ in acetone- $d_{6}$ 


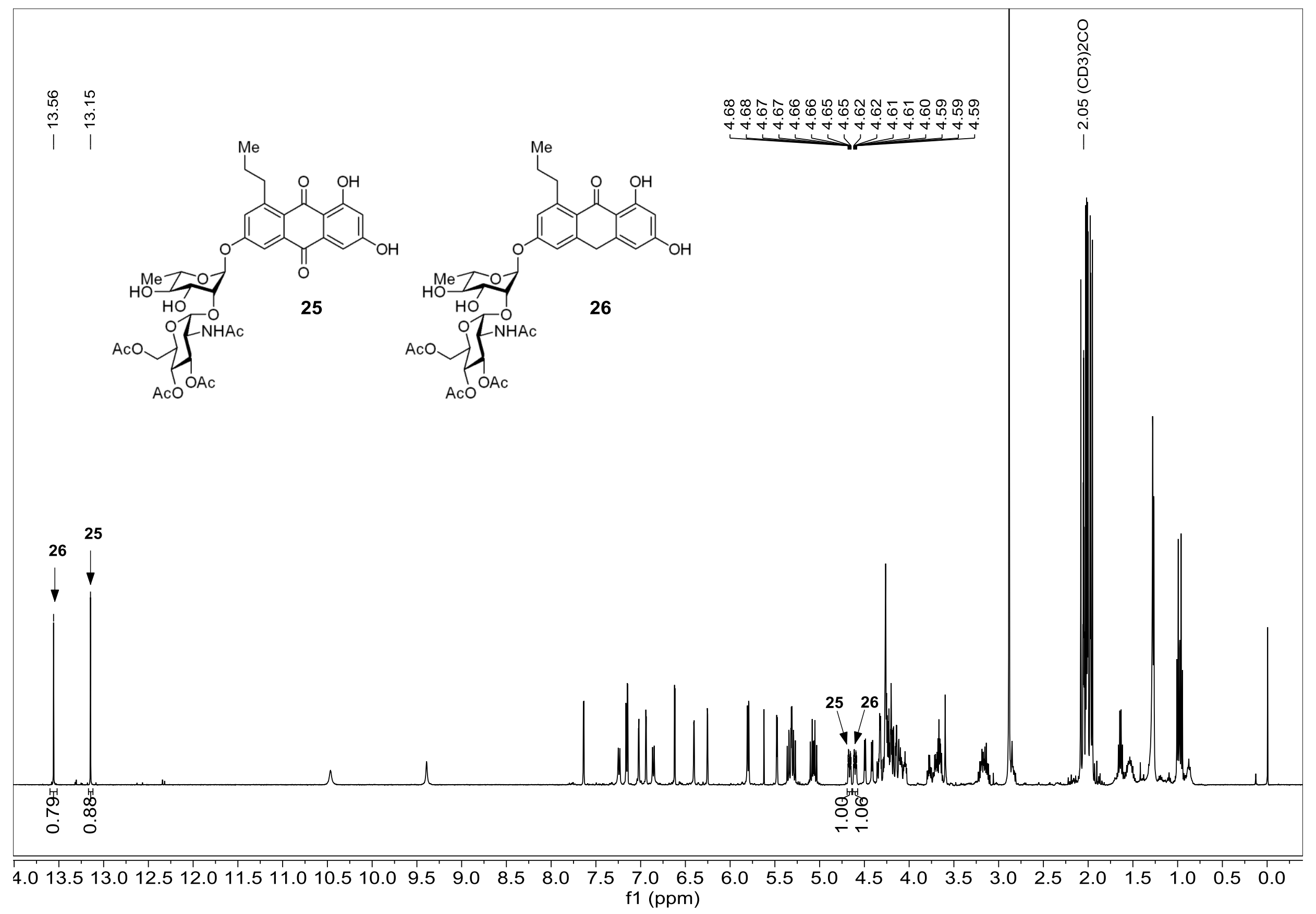

Figure S73. ${ }^{1} \mathrm{H}$ NMR spectrum of the mixture of 25 and 26 (500 MHz, acetone- $\left.d_{6}\right)$ 


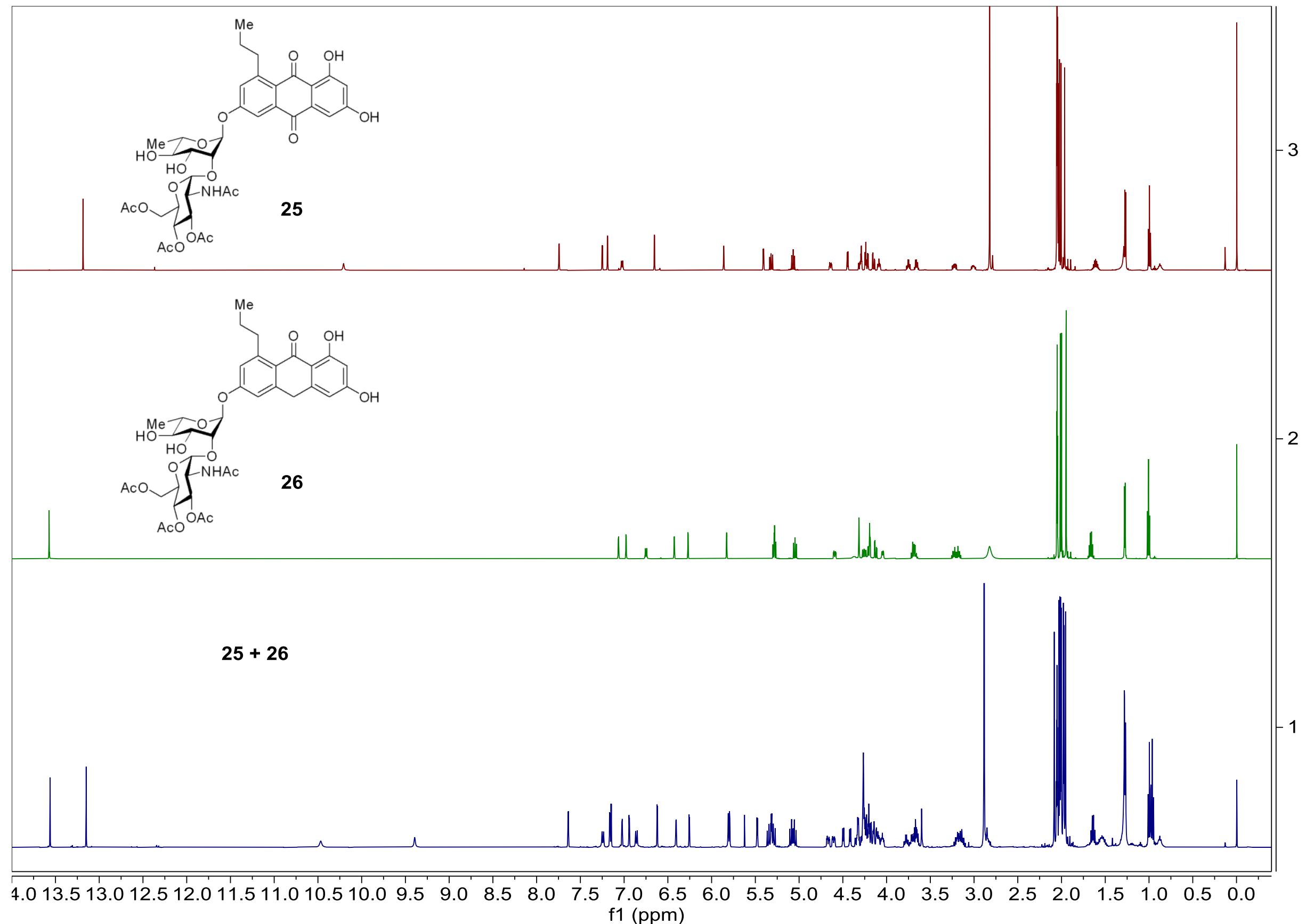

Figure S74. ${ }^{1} \mathrm{H}$ NMR spectrum of 25, 26 and their mixture in acetone- $d_{6}$ 


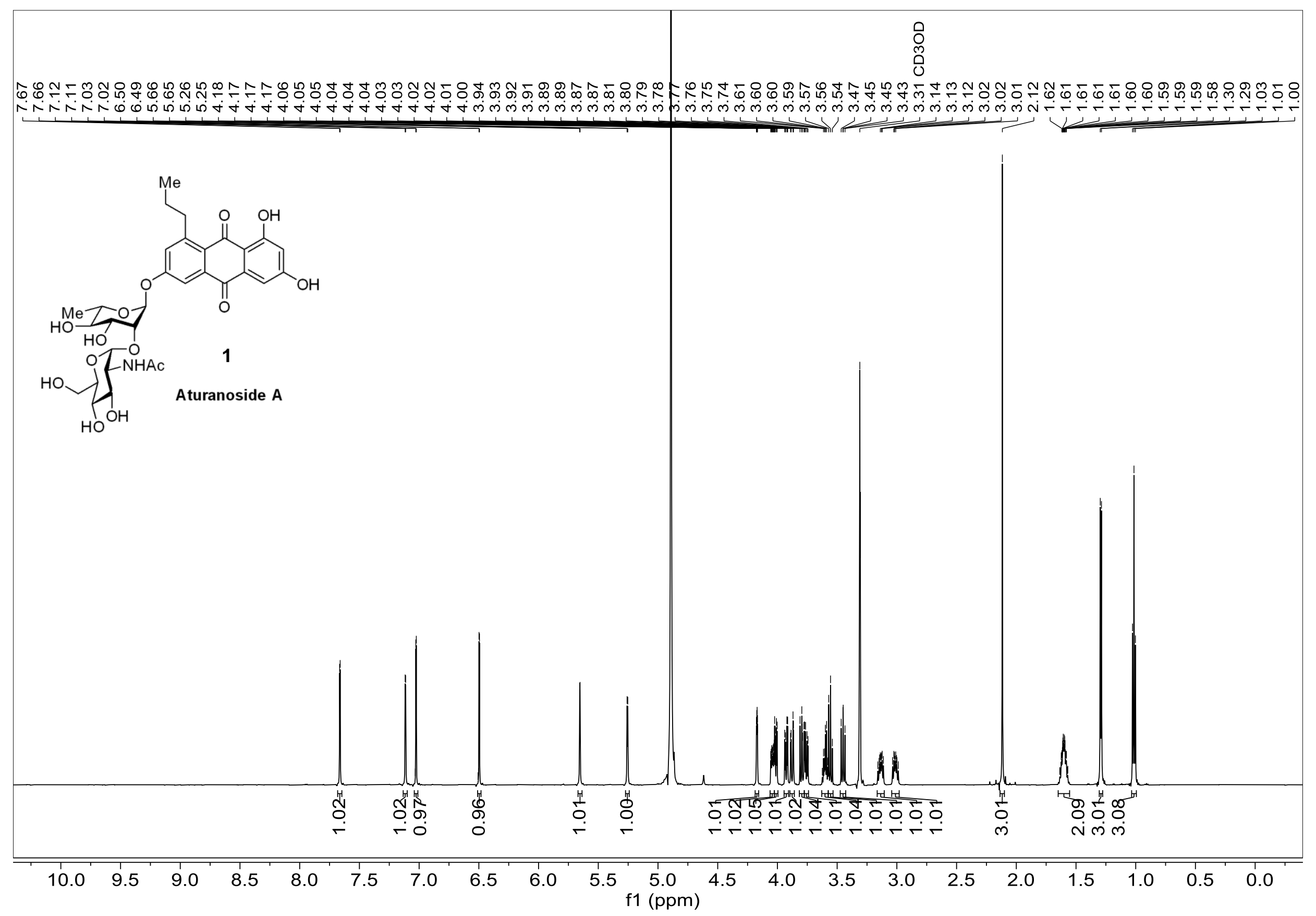

Figure S75. ${ }^{1} \mathrm{H}$ NMR spectrum of $1\left(600 \mathrm{MHz}, \mathrm{CD}_{3} \mathrm{OD}\right)$ 


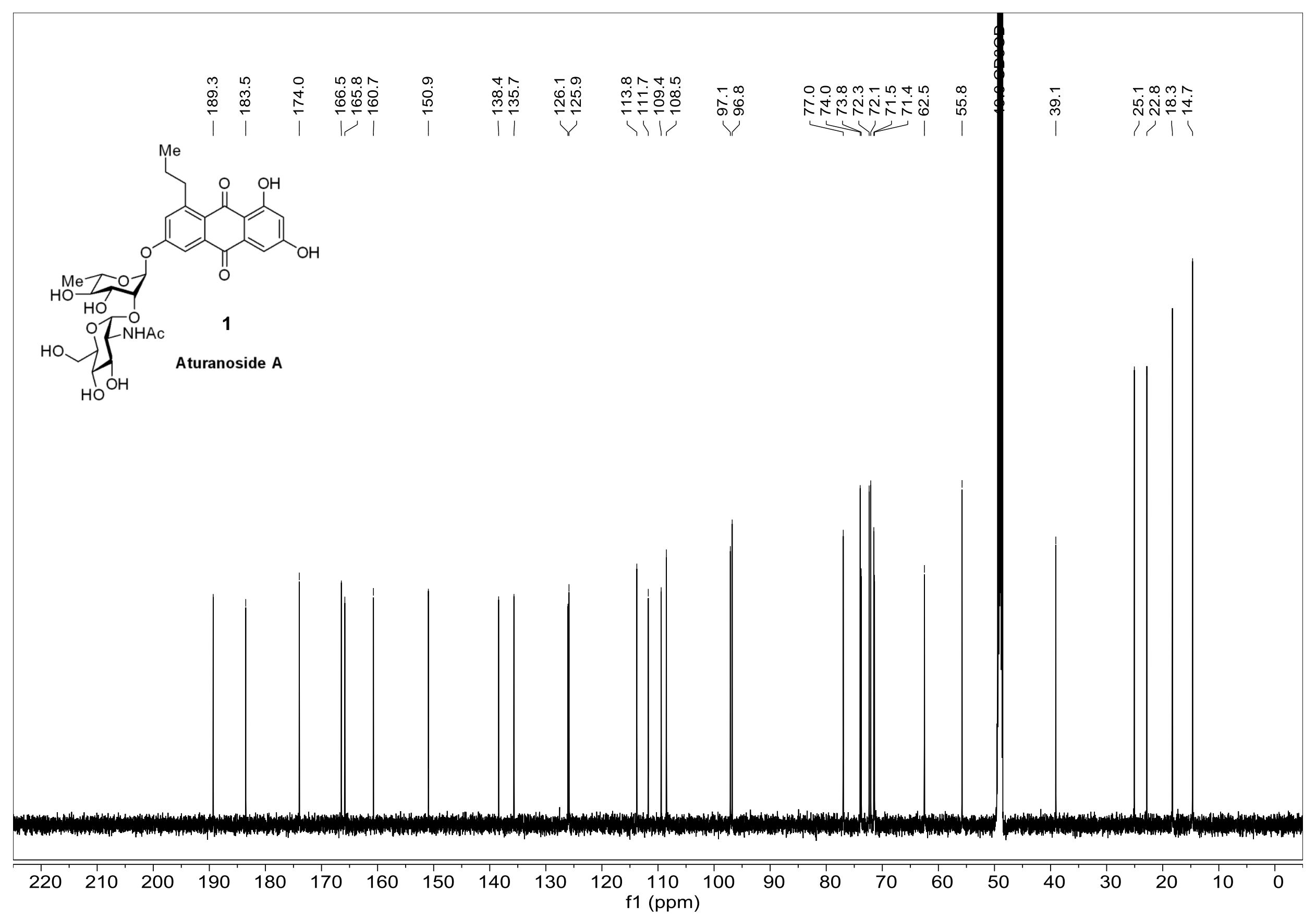

Figure S76. ${ }^{13} \mathrm{C}$ NMR spectrum of $\mathbf{1}\left(151 \mathrm{MHz}, \mathrm{CD}_{3} \mathrm{OD}\right)$ 


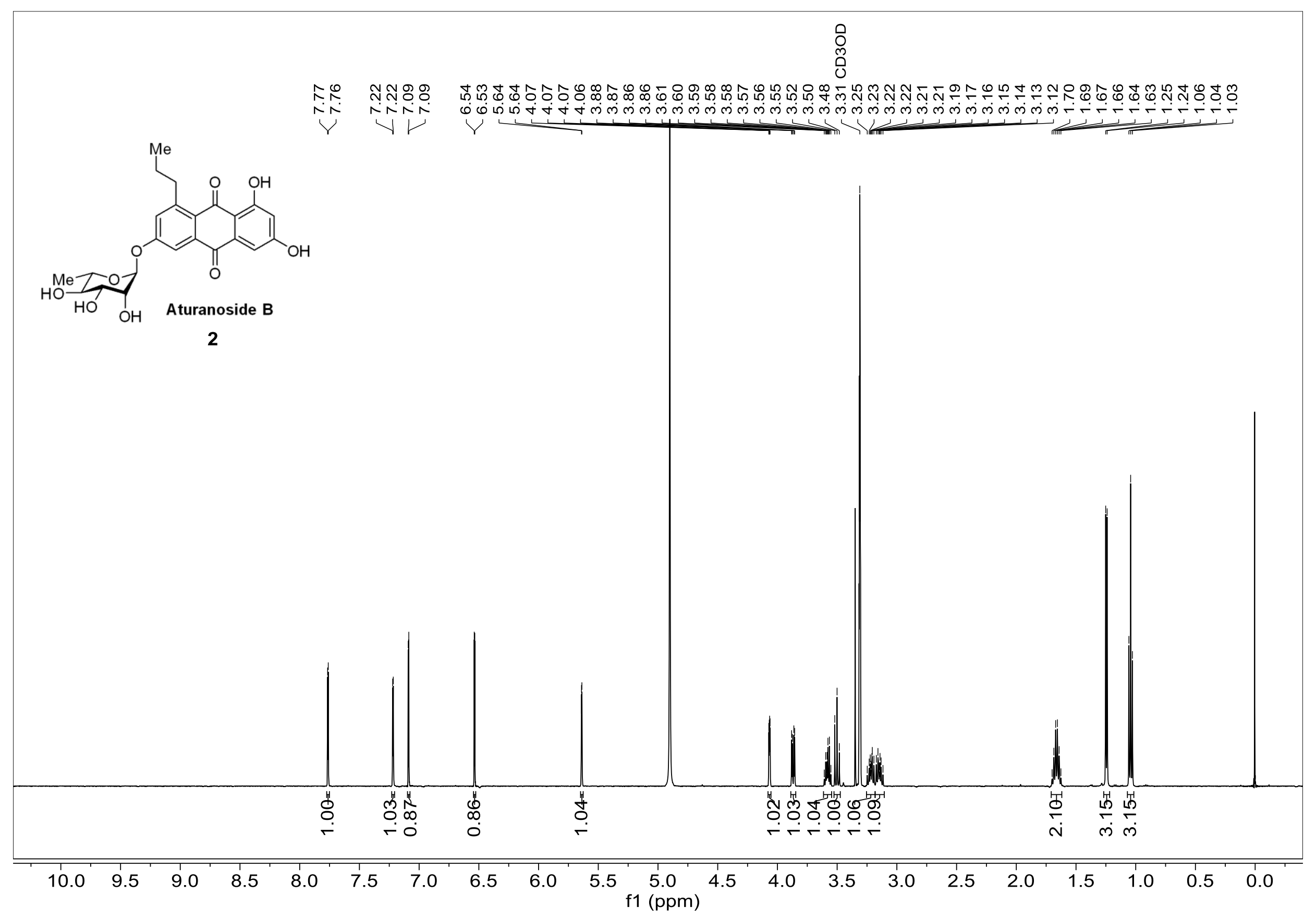

Figure S77. ${ }^{1} \mathrm{H}$ NMR spectrum of $2\left(500 \mathrm{MHz}, \mathrm{CD}_{3} \mathrm{OD}\right)$ 


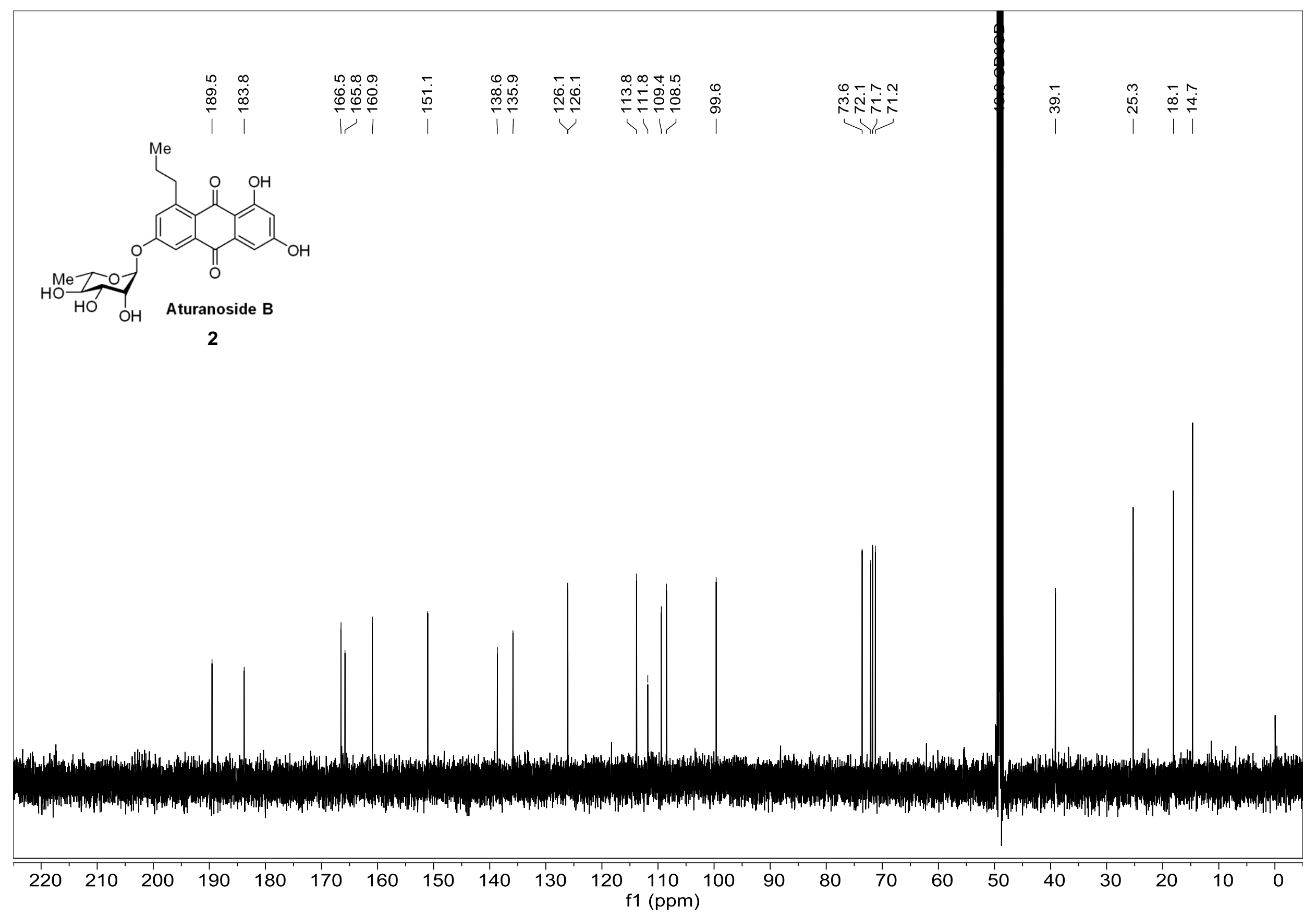

Figure S78. ${ }^{13} \mathrm{C}$ NMR spectrum of $2\left(126 \mathrm{MHz}, \mathrm{CD}_{3} \mathrm{OD}\right)$ 


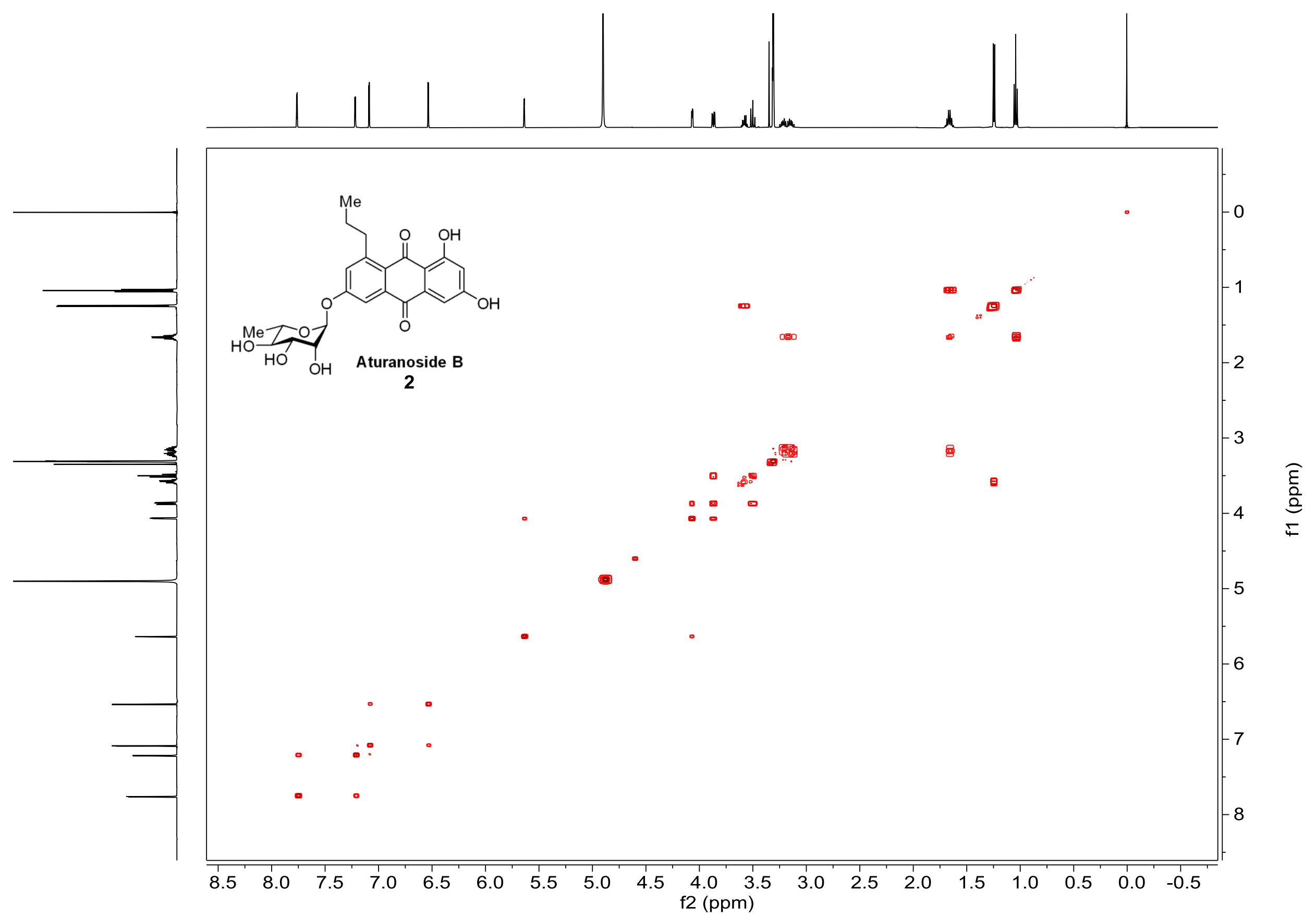

Figure S79. COSY spectrum of 2 in $\mathrm{CD}_{3} \mathrm{OD}$ 


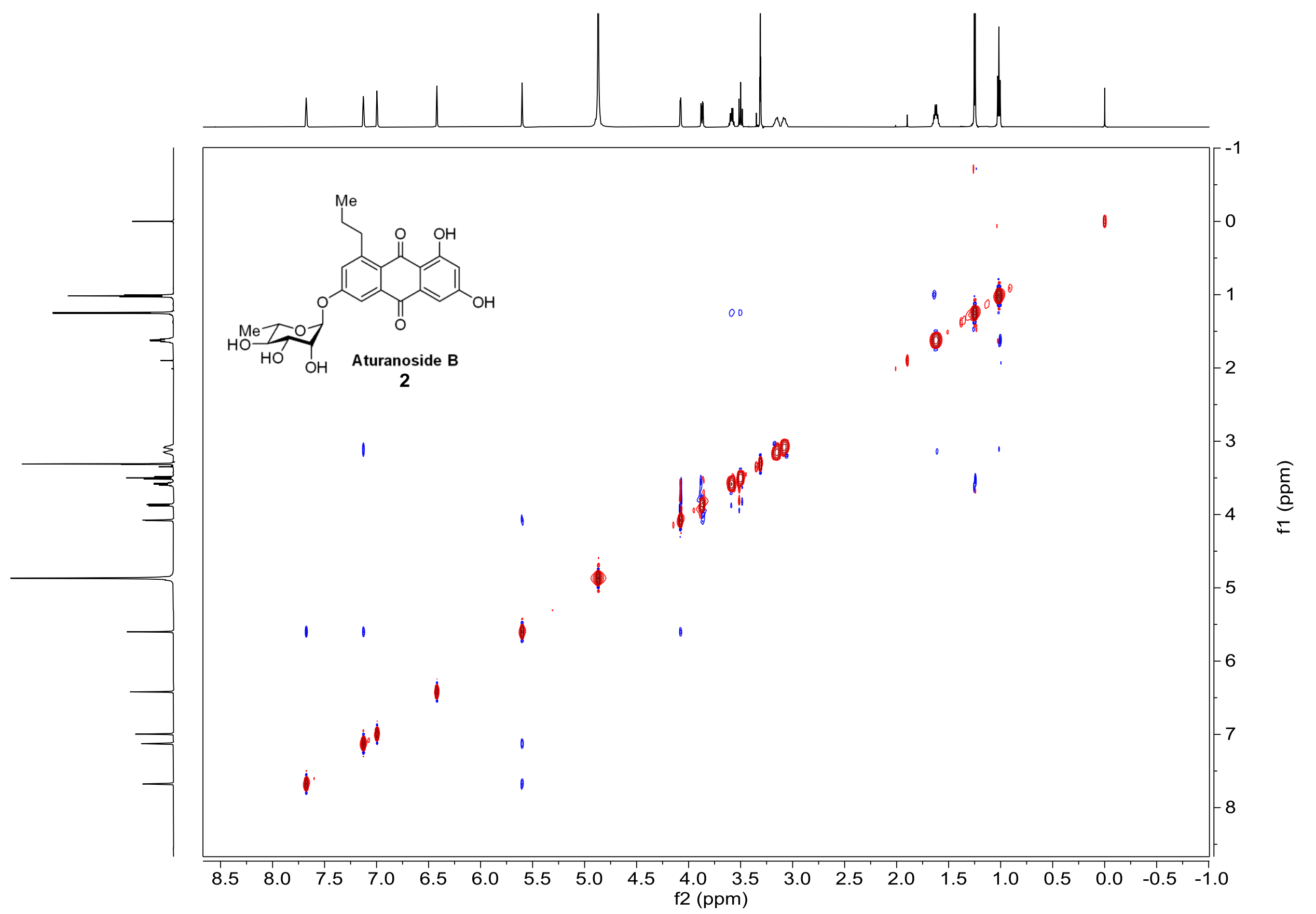

Figure S80. ROSEY spectrum of $\mathbf{2}$ in $\mathrm{CD}_{3} \mathrm{OD}$ 